\title{
Investigation of membrane fusion as a function of lateral membrane tension
}

\author{
Dissertation \\ for the award of the degree \\ Doctor rerum naturalium \\ of the Georg-August-Universität Göttingen \\ within the doctoral program \\ Physics of Biological and Complex Systems \\ of the Georg-August-University School of Science (GAUSS)
}

submitted by

Torben-Tobias Kliesch

from Wuppertal

Göttingen 2017 



\section{Thesis Committee}

Prof. Dr. Andreas Janshoff,

Institute for Physical Chemistry, Georg-August-Universität Göttingen

Prof. Dr. Tim Salditt

Institute for X-Ray Physics, Georg-August-Universität Göttingen

Dr. Florian Rehfeldt

Third Institute of Physics, Georg-August-Universität Göttingen

\section{Members of the Examination Board}

Prof. Dr. Andreas Janshoff

Institute for Physical Chemistry, Georg-August-Universität Göttingen

Prof. Dr. Tim Salditt,

Institute for X-Ray Physics, Georg-August-Universität Göttingen

Dr. Florian Rehfeldt,

Third Institute of Physics Georg-August-Universität Göttingen

\section{Further members of the Examination Board}

Prof. Dr. Peter Jomo Walla

Max Planck Institute for Biophysical Chemistry Göttingen, Department of Neurobiology

Prof. Dr. Bert de Groot

Max Planck Institute for Biophysical Chemistry Göttingen, Department of Theoretical and Computational Biophsics

Prof. Dr. Martin Suhm

Institute for Physical Chemistry, Georg-August-Universität Göttingen 


\section{Declaration}

I, Torben-Tobias Kliesch, herewith declare that my doctoral thesis entitled "Investigation of membrane fusion as a function of lateral membrane tension" has been written independently with no other sources and aids than quoted.

Göttingen, 2017

Torben-Tobias Kliesch 


\section{Contents}

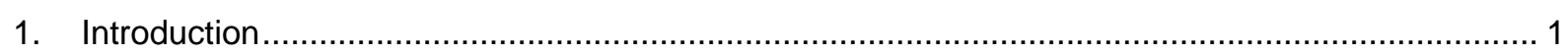

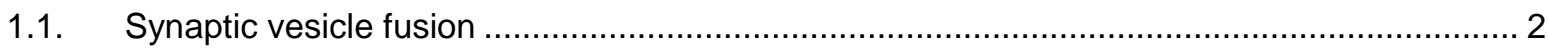

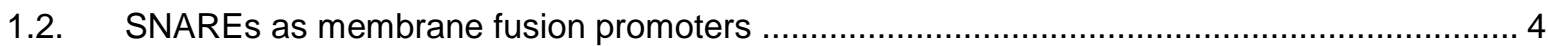

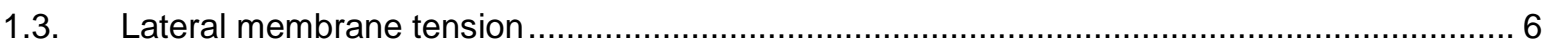

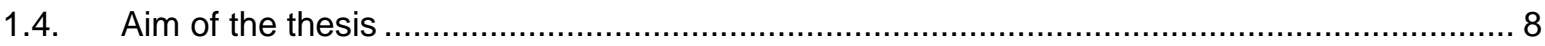

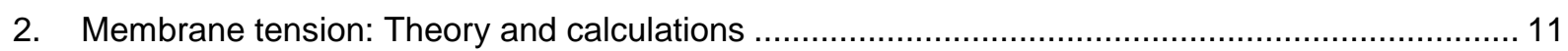

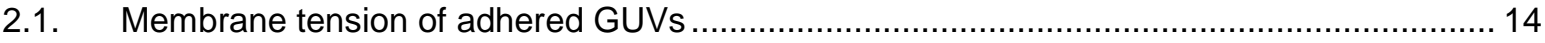

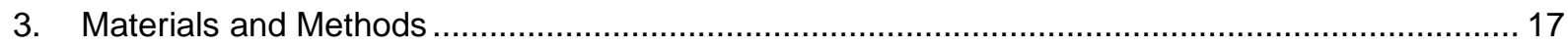

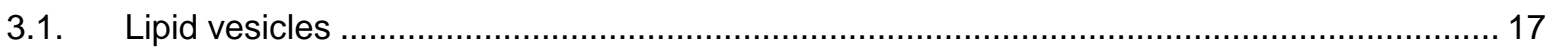

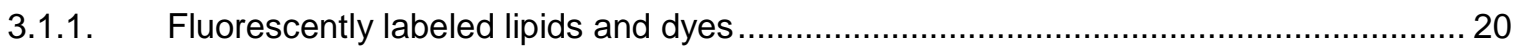

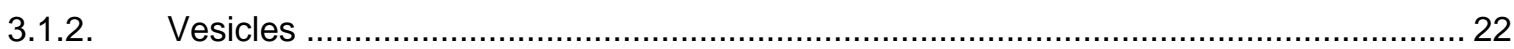

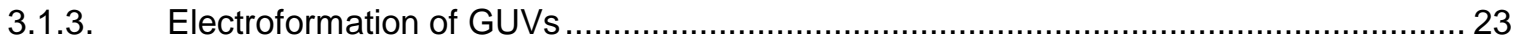

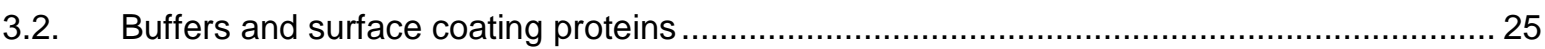

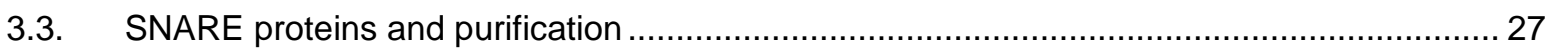

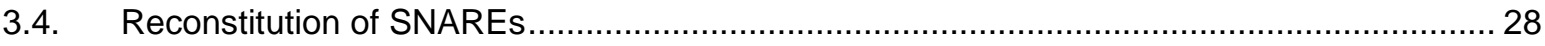

3.4.1. Dynamic light scattering - LUV size distribution ...................................................... 29

3.4.2. Vesicle Fusion assay after protein reconstitution ..................................................... 30

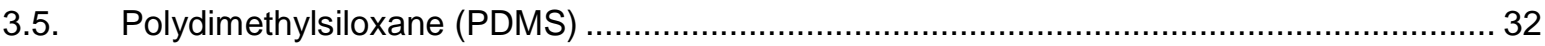

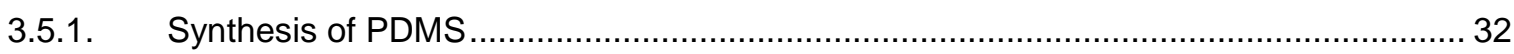

3.5.2. Design and fabrication of membrane stretcher device …............................................ 33

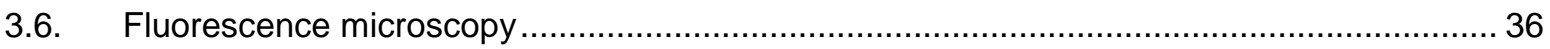

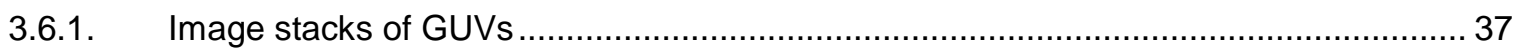

3.6.2. Fluorescence Recovery after Photobleaching ........................................................... 39

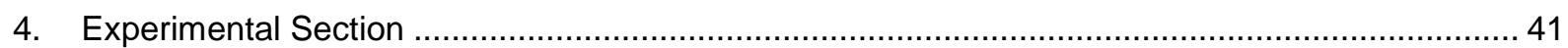

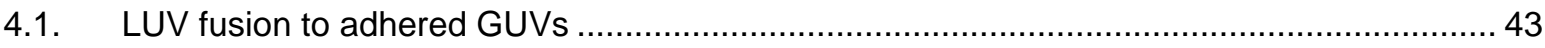

4.2. Extension of defined membrane areas on a PDMS substrate ......................................... 45

4.2.1. Preparation of membrane coated PDMS surfaces .................................................... 47

4.2.2. Measurement of supported lipid bilayer dilatation ...................................................... 48

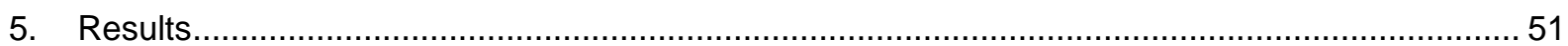




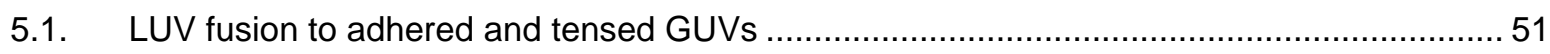

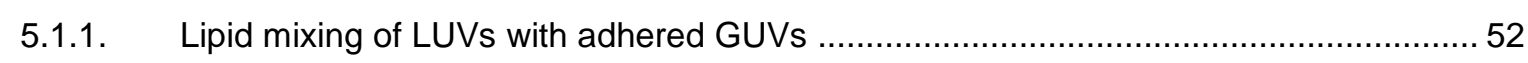

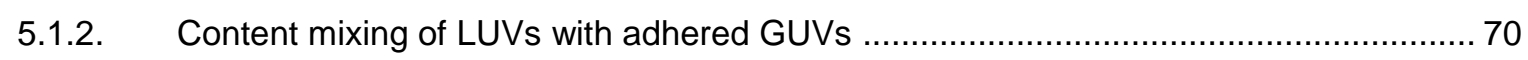

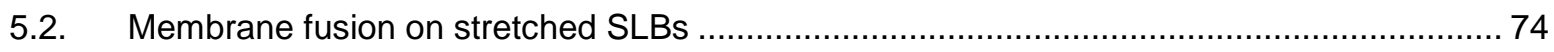

5.2.1. Dilatation of SLBs on hydrophilic PDMS surface ....................................................... 75

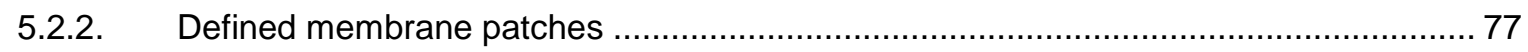

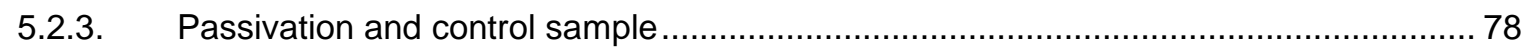

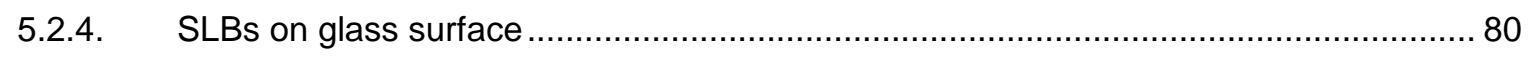

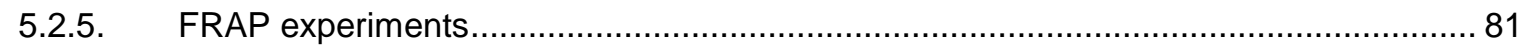

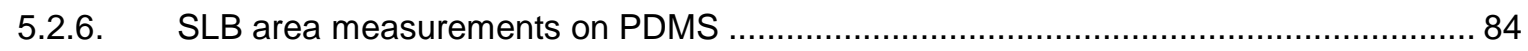

5.2.7. Fusion efficiency as a function of lateral membrane tension ...................................... 87

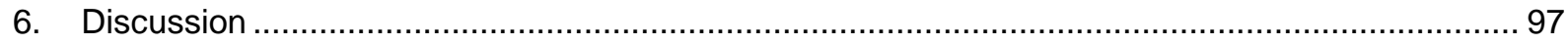

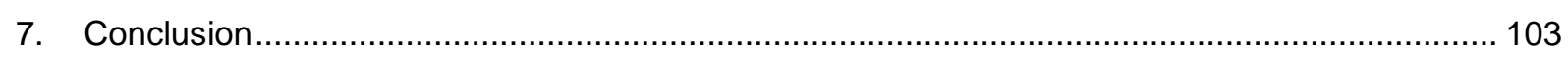

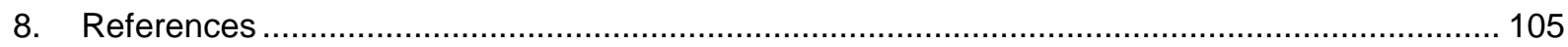

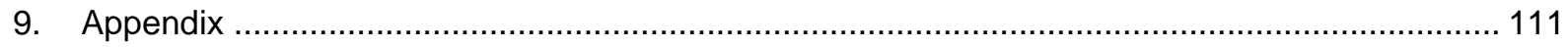

9.1. MATLAB scripts for the calculation of GUV membrane tension ..................................... 111

9.1.1. Calculation of GUV-membrane area change ….................................................. 111

9.1.2. Calculation of membrane tension as a function of fractional area change .................. 112

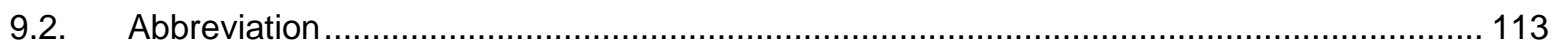

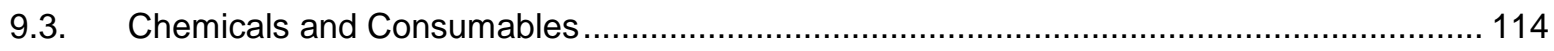

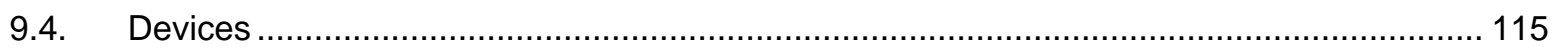

9.5. Software

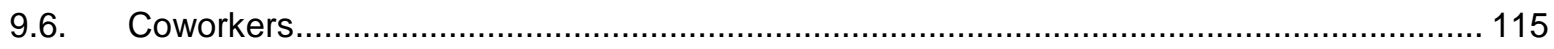

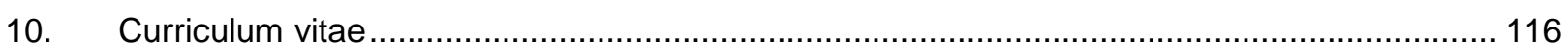

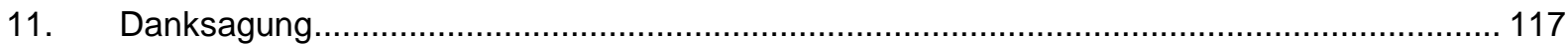




\begin{abstract}
Membrane fusion in native systems occurs on very short time scales and it has been proposed that lateral membrane tension of the presynaptic membrane is substantially increased to facilitate fusion. Proteins of the SNARE family (soluble $N$-ethylmaleimide-sensitive factor attachment protein receptors) are necessary to bring the membranes of a small vesicle and a cell membrane into close contact to promote fusion. It is proposed that membrane fusion is induced by, the formation of SNARE complexes, at the active zones in the plasma membrane that are composed many of different lipids and proteins. The connection of the cytoskeleton to the plasma membrane plays a pivotal role for the generation of membrane tension and the process of vesicle fusion. Molecular dynamics simulations have shown in the past that an increase in lateral tension facilitates fusion. To mimic the natural fusion process for e.g. in neuronal cells, different model systems with artificial membranes containing the SNARE core complex were investigated. In this study the tension-dependency of fusion using model membranes equipped with a minimal fusion machinery consisting of syntaxin 1, Synaptobrevin and SNAP 25 is addressed. With two artificial model systems the fusion of lipid membranes as a function of lateral membrane tension was investigated to seek a better understanding of fusion processes. The first model system contained giant vesicles that adhered on a functionalized glass surface. In the second model system, supported lipid bilayers were spread out of giant vesicles on a stretching device. The membrane tension of giant vesicles was adjusted through the adhesion area. Isolated patches of planar bilayers were formed from giant unilamellar vesicles and deposited on a dilatable polymeric sheet, which is part of a milli-fluidic stretching device allowing to adjust lateral tension in bilayer patches. Fusion of large unilamellar vesicles (LUVs) added to the solution was followed by fluorescence microscopy. The relative increase in fluorescence intensity, originating from the added LUVs, compared to the fluorescence intensity emitted from the giant vesicle membrane and planar bilayer patches served as a measure for fusion efficiency. It was found that fusion efficiency increases considerably with lateral tension and a threshold tension of $3.4 \mathrm{mN} \mathrm{m}^{-1}$ was identified at which fusion is boosted tremendously.
\end{abstract}





\section{Introduction}

Biological cell membranes and the compartments inside the cell are composed of lipid bilayers with a high proportion of proteins. These membranes serve as a barrier to maintain the function of each organelle and cell. Many processes in and between cells are important to sustain the whole function that keeps the organism alive. Small molecules, lipids and proteins have to be transported for their utilization through the barriers composed of lipid bilayers into the destined compartments of the cell. Fusion of small membranous organelles inside a cell and fusion of vesicles with the plasma membrane are one of the key steps in the secretory pathway for the transport of lipids, proteins and signaling molecules in eukaryotic cells. ${ }^{1-3}$ Elucidation of the function and regulation of lipid bilayer fusion is important for a deeper understanding of cell signaling and the general mechanism of membrane fusion.

The main fusion machinery in the secretory pathway, the lysosomal and endosomal transport of cellular materials are the proteins of the soluble $\mathrm{N}$-ethylmaleimide-sensitive-factor attachment receptors (SNAREs) that catalyze fusion by delivering the required free energy to reduce the energy barriers of fusion, the inter-bilayer repelling forces. ${ }^{4} \mathrm{It}$ is believed that SNAREs form a zippered structure of coiled coil alpha helical proteins between two opposing membranes bringing the outer leaflets into such close contact that the hydration barrier is reduced. ${ }^{5-8}$ SNAREs can be utilized to investigate the membrane fusion process in an artificial model system like the vesicle fusion assay where two vesicle populations are mixed to measure the fusion efficiency over time. ${ }^{9}$ The process of membrane fusion, where two adjacent lipid bilayers merge to shape a uniformed single lipid bilayer, is highly regulated by many different biological, chemical and physical factors in the membranes. Therefore, it is of the utmost importance to investigate the process of membrane fusion. In this thesis, the membrane fusion of vesicles to membranes with an artificially increased membrane tension was investigated to reveal that the lateral membrane tension in the target membrane regulates the fusion efficiency. 


\subsection{Synaptic vesicle fusion}

Membrane fusion of small vesicles occurs in every cell between the organelles and with the plasma membrane for the transport of lipids, proteins and other materials. The transport mechanism of neurotransmitters from one neuron to another is a good example of how signaling between cells is influenced by the fusion of membranes. Neuronal exocytosis of synaptic vesicles involves many different proteins including SNAREs, Rab, RIM, Munc18, and synaptotagmins. ${ }^{10} \mathrm{~A}$ large variety of proteins and proteins networks are involved in the process of synaptic vesicle fusion whereupon the lipid and protein composition of the synaptic vesicles in eukaryotic cells have been determined by TAKAMORI and JAHN et al. ${ }^{11}$ The whole process of docking and priming of synaptic vesicles at the presynaptic active zone membrane is mediated by at least five proteins (RIM, Munc13, RIM-BP, $\alpha$-liprin and ELKS) that form a large complex connecting $\mathrm{Ca}^{2+-}$-channels to the primed vesicles. ${ }^{12}$

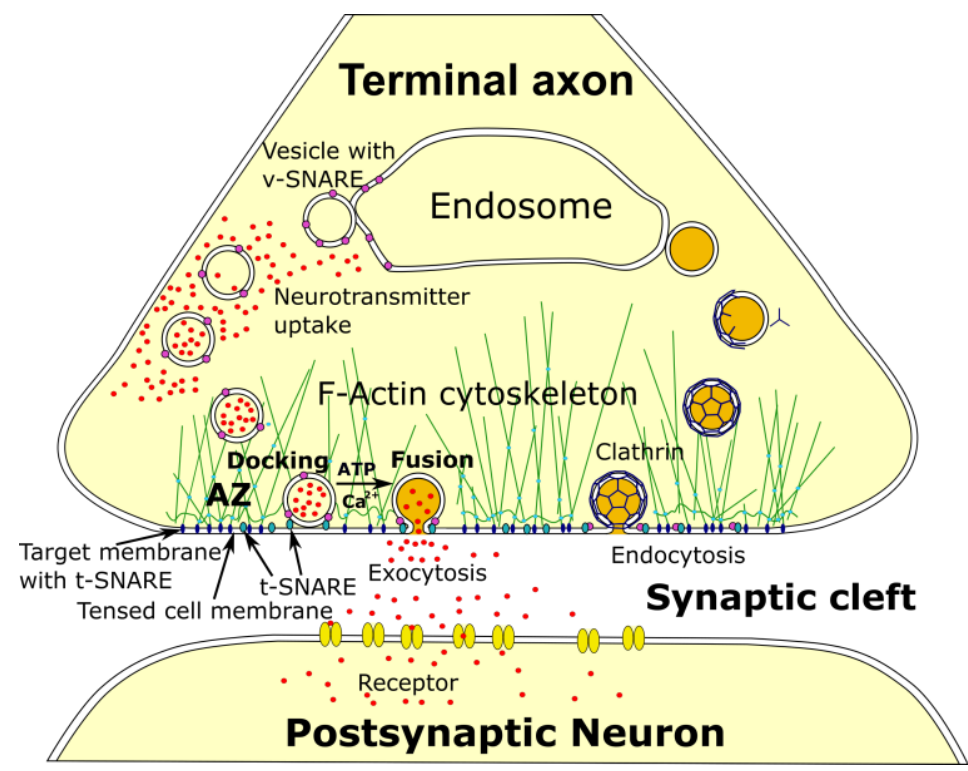

Figure 1.1. Illustration of a terminal axon of a neuron in the brain. Synaptic vesicles bud out of the endosome, take up neurotransmitters and then dock to the active zone (AZ) at the cell membrane which is supposed to bear a higher membrane tension that activates the membrane and reduces the energy barrier of membrane fusion. The filamentous-actin cytoskeleton at the AZ is connected to the plasma membrane and is supposed to induce a higher membrane tension that promotes SNARE-mediate synaptic vesicle fusion to the plasma-membrane. The endocytosis occurs through the mediation of clathrin that forms a skeleton around the fused synaptic vesicle and prevents depletion.

In the figure 1.1, a terminal axon of a neuron is illustrated that comprises the synaptic vesicle cycle starting at the endosome where the synaptic vesicles bud out and take up the neurotransmitter molecules. A vesicle pool in the terminal axon serves as a supply when the action potential is initiated by the uptake and flux of calcium ions. ${ }^{13}$ Then the synaptic vesicles dock and fuse with the plasma membrane at the active zone. Three mechanism at that stage occur at the active zone: Kiss and run, Full fusion to the plasma membrane, ultra-fast endocytosis. ${ }^{14-16}$ Kiss and run and ultrafast endocytosis recycles the synaptic vesicles for another cycle and the fast removal of the synaptic vesicles at the $A Z$ clears the region for further vesicles to fuse and release the neurotransmitter molecule at that place. 
Depletion of the synaptic vesicles occurs during the full fusion and incorporation of the lipid and protein material so that there is no recycling of the intact synaptic vesicle. Therefore, the ultrafast endocytosis is mediated by clathrin that coats the vesicles so that the geometry of the synaptic vesicles is maintained. Blocking of clathrin inhibits the recycling of synaptic vesicles..$^{17-18}$ In summary, the whole synaptic vesicle cycle is highly regulated to maintain the direction of the signal transduction and function of the connected neurons. The major process at the active zone is the fusion of the synaptic vesicle membrane with the plasma membrane for the release of neurotransmitters into the synaptic cleft so that the neurotransmitter can diffuse and dock to the opposing postsynaptic neuron whereby the signal is transmitted. If there is no direction of the synaptic vesicles to fuse with the AZ-membrane which is in near proximity to the opposing postsynaptic neuron then the synaptic vesicle will fuse with the plasma membrane anywhere in the terminal axon and the signal will be lost. The question about the active zone is which of their properties induce the high fusion rate. In literature it is known that the AZ-membrane is shown to enriched in cholesterol and connected to five proteins: RIM, Munc13, RIM-BP, $\alpha$-liprin and ELKS. ${ }^{12,} 19$ These five proteins are interconnected in the cytomatrix at the active zone and interact with synaptic vesicle proteins as well. 


\subsection{SNAREs as membrane fusion promoters}

The control and guidance of synaptic vesicles towards fusion is essential for signal transduction from the presynaptic neuron to the postsynaptic cell. The minimal fusion machinery in the secretory pathway is composed of the soluble $\mathrm{N}$-ethylmaleimide-sensitive-factor attachment receptor (SNARE) proteins that catalyze fusion. ${ }^{3}$ SNAREs assemble in a zipper-like fashion between two opposing membranes resulting in a bundle of four intertwined alpha helices, which brings the outer leaflets into close contact. ${ }^{5-7}$ Calcium-binding domains of synaptotagmin bind the calcium ions and initiate the formation of the SNARE complex that bridges the opposing membranes and finally fuse both membranes. ${ }^{20-23}$

The zippering of the SNARE core complex consisting of synaptobrevin, syntaxin and SNAP25 into the transmembrane-domain is responsible for the complex formation and free-energy release for membrane fusion. After membrane fusion the SNAREs form a zippered four bundle alpha helical structure that extends into the membrane. The membrane geometry of docked and hemi-fused membranes has been investigated by HERNANDEZ et al. using cryo-electron microscopy. ${ }^{9} \mathrm{~A}$ knock-out of an amino-acid in the synaptobrevin $\Delta 84$ resulted in a decreased fusion rate for small vesicles with a radius of around $20 \mathrm{~nm}$ and a complete inhibition of membrane fusion for larger vesicles with a radius of around $100 \mathrm{~nm}$. Interestingly, in an artificial vesicle fusion assays only the three above mentioned SNARE-motifs are necessary to achieve and detect full membrane fusion. ${ }^{24-25}$

In figure 1.2, the role of SNAREs in membrane fusion is illustrated. Two SNARE complexes at each side of the generated fusion pore are drawn figuratively because even one formed SNARE complex could lead to fusion. ${ }^{26-27}$ The mechanism of membrane fusion is consistently under debate and the main pathways are described in figure 1.2. At first a small point-like protrusion emerges (Figure 1.2 B) after the synaptobrevin from the vesicle starts to form a complex with the $\Delta \mathrm{N}$-complex composed of syntaxin and SNAP 25 from the flat membrane. Next, a hemi-fusion stalk is formed where the outer leaflet of both membranes fuse (Figure 1.2 C). ${ }^{27} \mathrm{~A}$ hemi-fusion diaphragm expands (Figure 1.2 D) until it ruptures at the side near the transmembrane domain of the SNAREs. Then the full fusion is accomplished while a fusion pore is formed (Figure 1.2 E). 5,28 The lipid material of the vesicle is able to diffuse into the flat

\section{- Syntaxin SNAP 25 - Synaptobrevin}

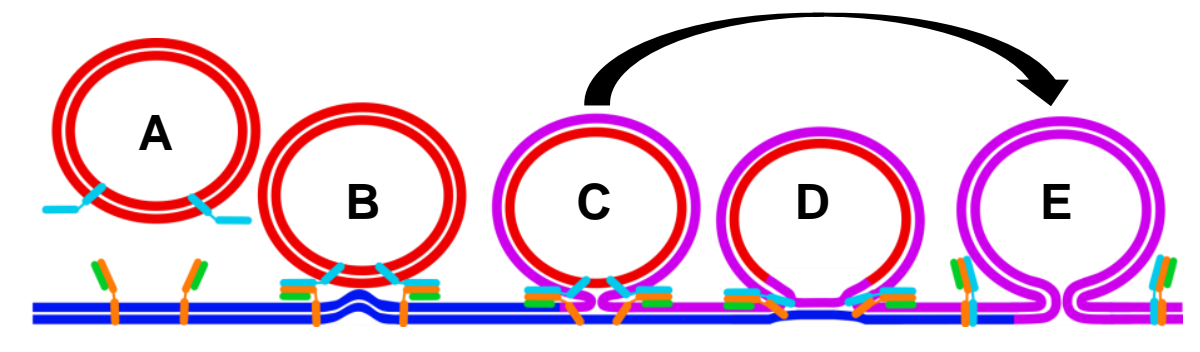

Figure 1.2. The mechanics of lipid bilayer fusion illustrated by the fusion of a vesicle to a flat membrane. A) The vesicle contains synaptobrevin and the flat membrane the $\Delta \mathrm{N}$-complex composed of syntaxin and SNAP 25. B) The SNARE complex starts to zipper and a protrusion stalk is formed. C) Both outer lipid layers fuse which is called hemi-fusion stalk. D) The hemi-fusion diaphragm expands until it ruptures. E) Full fusion of both lipid bilayer form the vesicle and the flat membrane either occurs through a fusion pore at the hemi-fusion diaphragm or directly at the hemi-fusion stalk. The zippering of the SNARE-complex into the transmembrane domain is finally finished and the whole complex now freely diffuses into the flat membrane. Incorporation of the vesicle lipid material into the flat membrane leads to the depletion of the vesicle. 
membrane so that a full incorporation could be gained by expansion of the flat membrane if there is no restriction to the deflation of the vesicle like a clathrin cytoskeleton in neuronal cells. The fusion geometry of the hemi-fusion and full fusion stalk has been investigated by myself and SAvić et al. in a combined computational and experimental study of membrane coated glass spheres. The diffusion between the hemi-fused and fully fused membrane coated glass spheres was measured through a fluorescence recovery after photobleaching (FRAP) measurement to yield a time constant of lipid diffusion across the membrane stalk. Comparison of experimental time constants of lipid diffusion "with Monte Carlo simulations suggest that geometrical restrictions due to a minimal contact zone reproduces the diffusion slowdown". 29 In a force-clamp approach with membrane coated sphere on an AFM-cantilever that was brought into close contact with a supported lipid bilayer the various intermediate fusion states of the SNARE proteins were investigated through a measurement of the life time distributions by OELKERS et al.. Their data suggest that "once the hydration barrier is conquered" the membranes fully fuse without a hemi-fusion intermediate state in the presence of SNAREs. ${ }^{30}$

It is well established that SNARE assembly provides the driving force for overcoming the energy barriers separating the intermediate states along the fusion pathway including stalk formation followed by the initial opening and the expansion of the fusion pore. ${ }^{6-7,31}$ The heights of these energy barriers are rate limiting for fusion but it is still unclear how exactly they arise. Depending on the assumption, in literature the energy barriers span a very broad range from $20 \mathrm{k}_{\mathrm{B}} T$ to $110 \mathrm{k}_{\mathrm{B}} T,{ }^{31-32}$ while a new experimental work form FRANÇOIS-MARTIN et al. in 2017 indicates that full fusion of membranes can be achieved by an activation energy of around $30 \mathrm{kB} T$ which is rather low but minimizes the spontaneous fusion and enables the fast membrane fusion with SNAREs. ${ }^{33}$ While the lipid composition is clearly important, local influences are exerted by mechanical stresses such as curvature and the finite lateral tension of the membrane ${ }^{34}$ The influence of curvature stress on fusion has been addressed in numerous studies, ${ }^{35-36}$ however, much less is known about how lateral membrane tension influences fusion kinetics. Lateral stress in the membrane exposes hydrophobic tails of the lipids and thereby reduces barriers associated with splaying of lipids and facilitates lipid insertion into the voids of stretched membranes. 


\subsection{Lateral membrane tension}

The fundamental theory of HELFRICH proposed that lipid bilayers are deformable elastic sheets underlying three main strains: Stretching, tilt, and curvature. ${ }^{37}$ Biological cells have to maintain their shape, structure and function whereas the cell membrane is permanently exposed to different stresses from the inside such as the cytoskeleton and the surrounding environment, others cells, solutions or stiff surfaces. Therefore, the shape of the cell membrane is changed permanently to maintain the surface area for the regulation of the cell volume and the lateral membrane tension. ${ }^{38-39}$ Many proteins can produce curvature stress and locally induce increased membrane tension for the exo- and endocytotic pathway and for the generation of membrane protrusions..$^{40-41}$

Lateral membrane tension is known to be involved in many biological processes comprising membrane trafficking, cell shape, adhesion, growth and motility. ${ }^{42-43}$ The lateral membrane tension describes the stresses of a lipid bilayer at every point along the surface interface and the surrounding medium, which is the cytosol in cells. ${ }^{44}$ Three main sources are responsible for mechanical tension in lipid bilayers:

(i) osmotic pressure between the cytosol and the surrounding medium of the cell or vesicle;38, 45-46 (ii) interaction of the cytoskeleton or with the plasma membrane; 47 and

(iii) adhesion forces to surfaces or other cells. ${ }^{44,48-49}$

In order to simulate tension in artificial systems one could in principle realize an osmotic imbalance, employ a defined adhesion strength to laterally dilate sessile liposomes or apply lateral stress directly to a supported bilayer. In this thesis, I use the two latter strategies of adhered liposomes and applied lateral stress to a supported bilayer to cover a broad range of tension values ranging from stress-free to lysis tension.

How is it possible to determine the lateral membrane tension cells or of vesicles? Evans et al. performed suction experiments with a micropipette that applied a tensile stress to a red blood cell by a suction of the membrane into the micropipette. The vesicle surface area change can be measured as a function of suction length into the micropipette..$^{50}$ With this micropipette aspiration technique the membrane tension of liposomes can be adjusted to measure the area compressibility modulus $K_{A}$ and bending modulus $k_{c}$. For example, with the micropipette aspiration of a 1,2-dioleoyl-sn-glycero-3-phosphocholine (DOPC) vesicle RAWICZ et al. calculated an area compressibility modulus $K_{A}=(0.265 \pm 0.018) \mathrm{N} / \mathrm{m} .{ }^{51}$ Indentation experiments with an AFM-cantilever tip are also a common technique to measure the membrane tension of various cells in vitro. ${ }^{52-53}$ SCHÄFER et al. used an AFM-Cantilever for the compression of a giant unilamellar vesicle (GUV) to apply higher membrane tension whereby an area compressibility modulus of $K_{\mathrm{A}}=(0.28 \pm 0.12) \mathrm{N} / \mathrm{m}$ was calculated for that lipid composition with DOPC as the main ingredient. ${ }^{51,54}$ Because of the comparable lipid composition that was used for the generation of vesicles in this thesis SCHÄFERS value was used for the calculation of membrane tension.

The main question this thesis is how the lateral membrane tension influences membrane fusion of for example synaptic vesicles to the active zone membrane which is suggested to bear a larger membrane tension than the other parts of the cells plasma membrane. In 2005 SHILLCOCK and LIPOWSKY postulated that fusion of bilayer membranes with vesicles is tension-induced and they performed molecular dynamics (MD) simulations that clearly show how an increase in lateral tension facilitates fusion. ${ }^{55}$ Their 
simulations showed that a tensionless vesicle only adheres to the lipid bilayer, while tension exerted in both opposing bilayers led to a higher rate of full membrane fusion events. If membrane fusion depends on the membrane tension then alternative fusion pathways will arise, for instance, tensed membranes may rupture at elevated tension, while at lower tension, the hemi-fused state can expand, thereby relaxing membrane tension by providing excess membrane area. The characteristic tensions found in the plasma membrane of eukaryotic cells are only in the range of $0.01-1 \mathrm{mN} / \mathrm{m}$ depending also on the cell type. Therefore, typical tension values found in the plasma membrane of cells are maybe not sufficient to substantially increase the fusion efficiency. Recent experimental studies from BRETOU et al. and WEN et al. addressed the hypothesis that membrane fusion in cells is powered by an increased lateral membrane tension, which was generated by hypo-osmotic conditions and the dynamics of the Factin cortex connected to the plasma membrane. ${ }^{47,56}$ The finite membrane tension generated by the underlying F-actin cortex resulted in full fusion of the vesicles with a depletion of the vesicles into the plasma membrane. ${ }^{47}$ Also cell-cell fusion is induced by invasive protrusions generated by F-actin from an attacking cell. A mechanosensory response by the actomyosin network of the receiving cell also provides tension in the plasma membrane as a resisting force to the invading cell membranes. ${ }^{57}$ The fact that membrane tension is generated by the connected, underlying cytoskeleton network of $\mathrm{F}$-actin supports the assumption that membrane tension is locally increased at defined membrane areas (e.g., active zones) to guide vesicle fusion by increasing hydrophobicity in the contact zone. Stretching of lipid bilayers increases the hydrophobicity because of the generated larger area for each lipid in the membrane so that voids between the headgroups of the lipids appear that expose the hydrophobic tails. Therefore, it is clear that the probability of successful fusion rises with tension but might run through an optimum since larger tension might inevitably lead to uncontrolled membranes rupture, while fusion probability decreases for smaller tensions as adhesion of the vesicle and hemi-fusion becomes more favorable. It is therefore suggested that the tension has to exceed a certain threshold value in order to induce fusion but might also level off at larger stress. In spite of all these indications, the hypothesis that elevated membrane tension increases vesicle fusion efficiency has not yet been verified experimentally. 


\subsection{Aim of the thesis}

The aim of this thesis is to prove the hypothesis that elevated membrane tension increases vesicle fusion. KOZLOV and CHERNOMORDIK suggested that a more sophisticated analysis of membrane tension is necessary to address the impact of tension in fusion assays for a better understanding of biological fusion processes. ${ }^{44}$ In order to prove the dependency of membrane fusion on elevated membrane tension, one needs to change the available membrane area within a small range from $0-5 \%$ of its initial area. For that purpose, two experimental setups were used to increase the surface area of membranes to induce elevated membrane tension and to measure the fusion efficiency of large unilamellar vesicles (LUVs).

In the first experimental approach, a functionalized glass surface was utilized to adhere GUVs on the surface whereby the adhesion area was adjusted to yield defined ranges of GUV-membrane tensions. ${ }^{49}$ Through the addition and incubation of LUVs in the surrounding solution to the adhered GUVs on the substrate surface, membrane fusion was mediated by the minimal fusion system consisting of SNAREs comprising synaptobrevin (1-116), syntaxin1A (183-288), and SNAP-25 (1-206). Like in synaptic vesicle fusion to the active zone plasma membrane LUVs were equipped with synaptobrevin (1-116) and the GUV-membranes were equipped with the $\Delta$ N49-complex consisting of syntaxin1A (183-288), SNAP 25 (1-206) and Syb2 (49-96). By measurement of image stacks with a confocal laser scanning microscope the three dimensional geometry of each GUV was determined for the calculation of membrane tension. For the detection of LUV fusion to the adhered GUVs, two fluorescent dye systems were used: Content and lipid mixing. LUVs with a water soluble dye in their content led to a fluorescence increase at the encapsulated GUV content. Through the use of two different fluorescently labeled lipid dyes, one in the GUV- and the other one in the LUV-membrane, it was possible to measure the fluorescence intensity of the LUV dye at the freestanding GUV-membrane and the adhesion site. Lipid mixing of the LUVs with the GUVs was also detected at the adhesion site of the GUVs through FRAPmeasurements for the LUV dye. With this technique it was possible to compare the fusion efficiency on GUV-membranes with high and low membrane tensions.

With a second experimental approach, the LUV fusion efficiency was tested on supported lipid bilayers (SLBs). Here, global tension was generated using a milli-fluidic device as a substrate, whereby the full range from slightly negative to lysis tension could be measured with high accuracy. Stretching and compression of lipid bilayers on an elastic polydimethylsiloxane (PDMS) sheet was first established by Staykova et al. using changing air pressure in a chamber with a thin PDMS layer on top. ${ }^{58-59}$ This experimental approach was particularly inspired by the work of $\mathrm{HUH}$ et al., who investigated the mechanical properties of cells on stretchable substrates to mimic the biological environment in an organ that is exposed to mechanical deformation. ${ }^{60-61}$ Replica modeling of these constructions provided a device bearing a thin PDMS sheet spanning between two side channels, where air pressure could be reduced to achieve an increased surface area on the sheet. This in turn was used to increase the area of adhered lipid bilayers that were previously spread from GUVs. Membrane tension was adjusted by applying a vacuum to adjuvant channels of this device and the membrane area change of membrane patches was measured using an optical microscope in conjunction with the thresholding technique of $\mathrm{LI}$ et al. ${ }^{62}$ SNAREs were used as membrane fusion promoters as described for the first experimental 
approach. With this experimental setup, one could monitor the area change of each membrane patch after stretching the substrate and document the fusion of LUVs added to the solution directly by fluorescence microscopy.

The experimental work of this thesis provides an immense contribution to the investigation of membrane fusion because mechanical properties of fluid and elastic sheets like membranes are a challenging measurement task and therefore have been investigated mainly in computer simulations but with the new techniques presented in this thesis it is now possible to precisely adjust membrane tension in the range of $0.1 \mathrm{mN} / \mathrm{m}$ up to the lysis tension of $10 \mathrm{mN} / \mathrm{m}$ of adherent vesicles and lipid bilayers on a deformable support. This system allows for the first time to quantitatively investigate the impact of membrane tension on vesicle fusion for the first time. 


\section{Membrane tension: Theory and calculations ${ }^{1}$}

The aim of this thesis is to generate membrane tension by applying a mechanical stress to dilate the membrane area. Adhesion of GUVs on a functionalized surface results in an increased membrane area compared to the surface area of freely floating GUVs because the adhesion energy leads to a deformation. The area change of GUVs due to adhesion and the involved membrane tension that arises by the increased membrane area has been studied by GLEISNER et al. by introducing the experimental method of changing the adhesion area as a function of the divalent ion concentration. A brief overview to the main equations that were used to calculate the membrane tension of adhered GUVs and SLBs is represented in this section. ${ }^{49}$

Stretching of supported lipid bilayers on PDMS surfaces also increases membrane area. The stretching or compression energy $E_{\text {stretch }}$ of a membrane by starting with an initial optimal area $A_{0}$ of the membrane is defined by

$$
E_{\text {stretch }}=\frac{1}{2} K_{\mathrm{A}} \frac{\left(A-A_{0}\right)^{2}}{A_{0}}
$$

comprising the membrane area compressibility modulus $K_{\mathrm{A}}$ and the change in membrane area $\Delta A=\left(A-A_{0}\right)$ with $A$ for the area of the dilated membrane, and $A_{0}$ for the area of the initial area before dilatation. ${ }^{49,63}$

The question is, what is the definition of lateral membrane tension $\tau$ ? Per definition, it is the derivative of the stretching energy through membrane area change: 63

$$
\frac{\partial E_{\text {stretch }}}{\partial A}=\tau=K_{\mathrm{A}} \frac{A-A_{0}}{A_{0}}=K_{\mathrm{A}} \frac{\Delta A}{A_{0}} .
$$

The membrane tension was also found experimentally by Evans et al. using a micropipette that applied a tensile stress to a red blood cell by a suction of the membrane into the micropipette. ${ }^{50}$ However, equation (2) neglects membrane undulations that arise due to thermal fluctuations in the membrane and thus equation (2) is only representative for large membrane tension where membrane undulations are suppressed by the membrane rigidity or through adhesion of SLB on a PDMS substrate. ${ }^{64}$ Therefore, equation (3) for the tension of a vesicle membrane was found by Evans et al. that comprises the thermal undulations of the membrane and the pretension $\sigma_{0}$ of a membrane prior to adhesion, stretch or compression. ${ }^{49,64-65}$

$$
\frac{\Delta A}{A_{0}}=\frac{\sigma-\sigma_{0}}{K_{\mathrm{A}}}+\frac{k_{\mathrm{B}} T}{8 \pi K} \ln \left(\frac{\sigma}{\sigma_{0}}\right)
$$

\footnotetext{
${ }^{1}$ Partly published in: Gleisner, M.; Kroppen, B.; Fricke, C.; Teske, N.; Kliesch, T.-T.; Janshoff, A.; Meinecke, M.; Steinem, C., Epsin N-terminal Homology Domain (ENTH) Activity as a Function of Membrane Tension. Journal of Biological Chemistry 2016, 291 (38), 19953-19961.
} 
The first term represents area dilatation according to Hooke's law and the second term represents the thermal undulations of the membrane and is composed of the BOLTZMANN constant $k_{\mathrm{B}}$, the temperature $T$, the bending rigidity $\kappa$ and a logarithmic term comprising the tension $\sigma$ and pre-stress tension $\sigma_{0}$.

With equation (3) the membrane tension was calculated for GUVs. A detailed overview to the MATLAB scripts (MathWorks, Natick, Massachusetts, USA) for the calculation of membrane tension of adhered GUVs given in the appendix of this thesis.

For the calculation of membrane tension $\tau$ of supported lipid bilayers equation (6) was used with the assumption that the pre-stress tension $\sigma_{0}$ of the membrane is orders of magnitude smaller (in the range of $10^{-6}$ to $\left.10^{-4} \mathrm{mN} / \mathrm{m}\right)$ than the applied tensions between $(0.1-10 \mathrm{mN} / \mathrm{m})$. Therefore, membrane tension in this thesis refers to the tension $\tau$ which comprises applied tension $\sigma$ by subtraction of pre-stress tension $\sigma_{0}$ originating form equation (4):

$$
\begin{gathered}
\sigma=\sigma_{0}+K_{\mathrm{A}} \frac{\Delta A}{A_{0}} \\
\tau=\sigma-\sigma_{0} \\
\tau=K_{\mathrm{A}} \frac{\Delta A}{A_{0}}
\end{gathered}
$$

The tension of each individual membrane patch was calculated by assuming an area compressibility modulus of $K_{\mathrm{A}}=0.28 \mathrm{~N} / \mathrm{m}$ for this lipid composition which was previously measured by the compression of GUVs of similar lipid composition with an AFM by SCHÄFER et al.51, 54

To visualize the membrane tension depending on the area change by stretching of the underlying polymer support, figure 2.1 shows the graph for equation (6) $\left(K_{\mathrm{A}}=0.28 \mathrm{~N} / \mathrm{m}\right)$. The violet lines define the point on the tension line at which the theoretical lysis tension of $10 \mathrm{mN} / \mathrm{m}$ is reached. At $3.6 \%$ area change and above that value the area increase of the membrane is higher than the lysis tension (highlighted square) so that possibly measured higher membrane area changes above that value have to be analyzed carefully upon possible defects in the membrane that are challenging to detect by the used thresholding technique of $\mathrm{LI}$ et al. All measured membrane area changes therefore should be between zero and 3.6\%. Negative area changes also occurred due to rupturing and defects but these negative area changes correspond to a relaxed membrane which here is defined as zero membrane tension. 


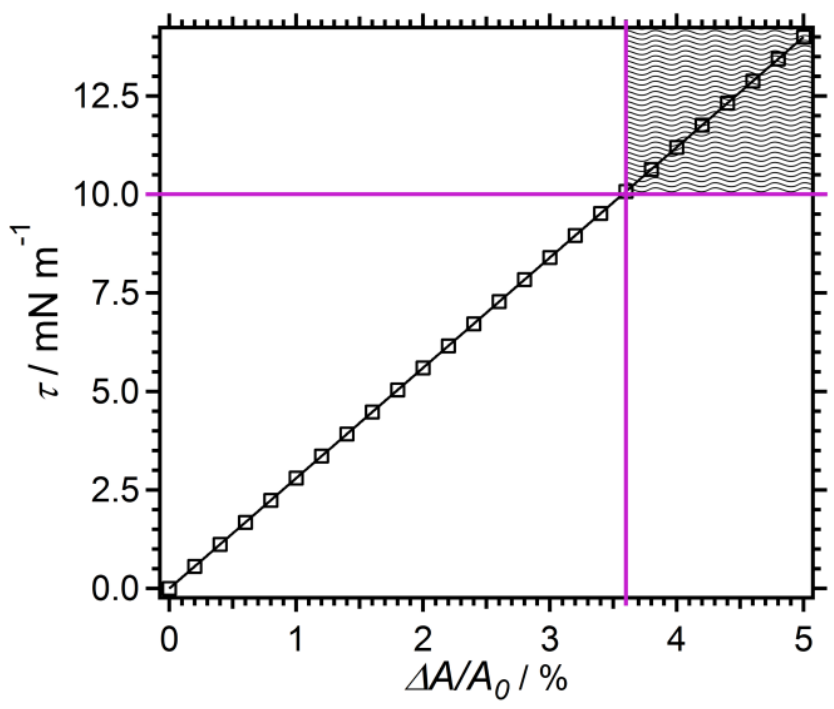

Figure 2.1. Calculated membrane tension of a SLBs depending on the membrane area increase induced by adhesion of the GUVs on the surface or by a mechanical stress induced by the dilated polymer support. The lysis tension of $10 \mathrm{mN} / \mathrm{m}$ is reached at an area increase of around $3.6 \%$ compared to the initial membrane area 


\subsection{Membrane tension of adhered GUVs}

For this thesis, the calculation of GUV membrane tension is a prerequisite for the investigation of membrane fusion on dilated membranes. The following the geometrical conditions led to the derived and explained equations in this chapter which were subject in the study of myself and GLEISNER et al.. The geometrical values needed for the calculation of the membrane tension of GUVs can be measured with a confocal microscopy by taking image stacks.

At first, the geometrical conditions of adhered GUVs have to be considered for the calculation of surface area prior and during to adhesion of the GUV. It is assumed that the vesicle adheres to the surface with a defined contact radius $R_{\mathrm{i}}$ without changing the volume so that the surface area has to increase.

A

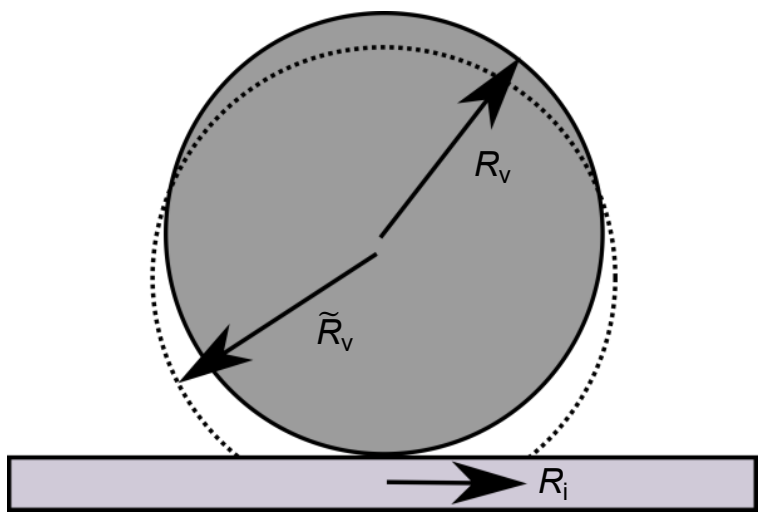

B

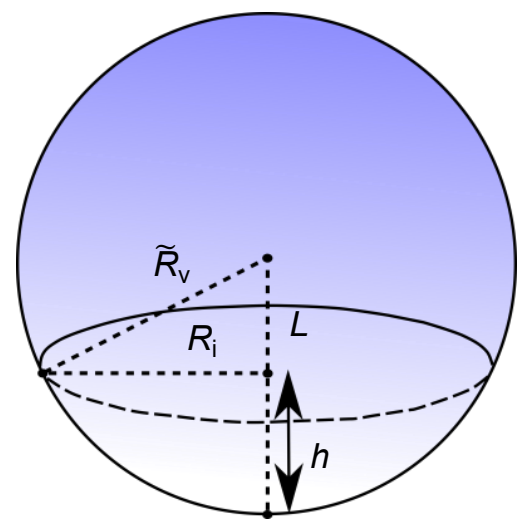

Figure 2.2. Schematic illustration of GUV adhesion. A) A floating GUV with the vesicle radius $R_{\mathrm{v}}$ touches the surface. Adhesion of the GUV leads to an increased vesicle radius $\widetilde{R}_{\mathrm{v}}$ and to an adhesion radius $R_{\mathrm{i}}$. B) The sphere where a spherical cap with the height $h$ is cut off represents the geometry of the adhered GUV on the surface.

In figure 2.2 the GUV adhesion is shown schematically. The vesicle radius $R_{\mathrm{v}}$ changes to $\widetilde{R}_{\mathrm{v}}$ due to adhesion. The vesicle behaves as a spherical cap by neglecting bending contributions from the edges. ${ }^{54}$ The height of the missing cap cut-off due to adhesion is

$$
h=\widetilde{R}_{\mathrm{v}}-\sqrt{\widetilde{R}_{\mathrm{v}}^{2}-R_{\mathrm{i}}^{2}} .
$$

The radius of the adhered vesicle can be expressed as

$$
\widetilde{R}_{\mathrm{v}}=\frac{1}{2}(L+h)
$$

with the height $L$ of the vesicle, $h$ for the vesicle cap as described before and $R_{\mathrm{i}}$ for the contact radius. 
Because the volume of the GUV stays constant the volume of the freely floating GUV is equal to the adhered one:

$$
\frac{4}{3} \pi R_{\mathrm{v}}^{3}=\frac{4}{3} \pi \widetilde{R}_{\mathrm{v}}^{3}-\frac{\pi h}{6}\left(3 R_{\mathrm{i}}^{2}+h^{2}\right)
$$

Therefore, the vesicle radius $\widetilde{R}_{\mathrm{v}}$ can be achieved by solving the following equation analytically:

$$
\begin{aligned}
\widetilde{R}_{\mathrm{v}}=\frac{R_{\mathrm{i}}^{4}}{16 R_{\mathrm{v}}^{3}} & +\frac{R_{\mathrm{i}}^{8}}{16 R_{\mathrm{v}}^{3^{3}} \sqrt{R_{\mathrm{i}}^{12}+128 R_{\mathrm{i}}^{6} R_{\mathrm{v}}^{6}+2048 R_{\mathrm{v}}^{12}+16 \sqrt{R_{\mathrm{i}}^{18} R_{\mathrm{v}}^{6}+80 R_{\mathrm{i}}^{12} R_{\mathrm{v}}^{12}+2048 R_{\mathrm{i}}^{6} R_{\mathrm{v}}^{18}+16348 R_{\mathrm{v}}^{24}}}}+ \\
& \frac{\sqrt[3]{R_{\mathrm{i}}^{12}+128 R_{\mathrm{i}}^{6} R_{\mathrm{v}}^{6}+2048 R_{\mathrm{v}}^{12}+16 \sqrt{R_{\mathrm{i}}^{18} R_{\mathrm{v}}^{6}+80 R_{\mathrm{i}}^{12} R_{\mathrm{v}}^{12}+2048 R_{\mathrm{i}}^{6} R_{\mathrm{v}}^{18}+16348 R_{\mathrm{v}}^{24}}}}{16 R_{\mathrm{v}}^{3}}
\end{aligned}
$$

In experiments it is only possible to determine the radius $\widetilde{R}_{\mathrm{v}}$ of the adhered GUV from images taken with a confocal microscope because the initial radius $R_{\mathrm{v}}$ cannot be achieved by taking image stacks (3D) of floating GUVs. Therefore, the initial vesicle radius $R_{\mathrm{v}}$ can be obtained from the following equation:

$$
R_{\mathrm{v}}=\sqrt[3]{\widetilde{R}_{\mathrm{v}}^{3}-\frac{h}{8}\left(3 R_{\mathrm{i}}^{2}+h^{2}\right)}
$$

Finally, the fractional area change $\alpha$ of the vesicle can be computed with $A_{0}=4 \pi R_{\mathrm{v}}^{2}$ :

With a vesicle surface area

$$
\alpha=\frac{\Delta A}{A_{0}}=\frac{A_{\mathrm{v}}-A_{0}}{A_{0}}
$$

$$
A_{v}=\pi R_{\mathrm{i}}^{2}+4 \pi \widetilde{R}_{\mathrm{v}}^{2}-2 \pi \widetilde{R}_{\mathrm{v}} h
$$
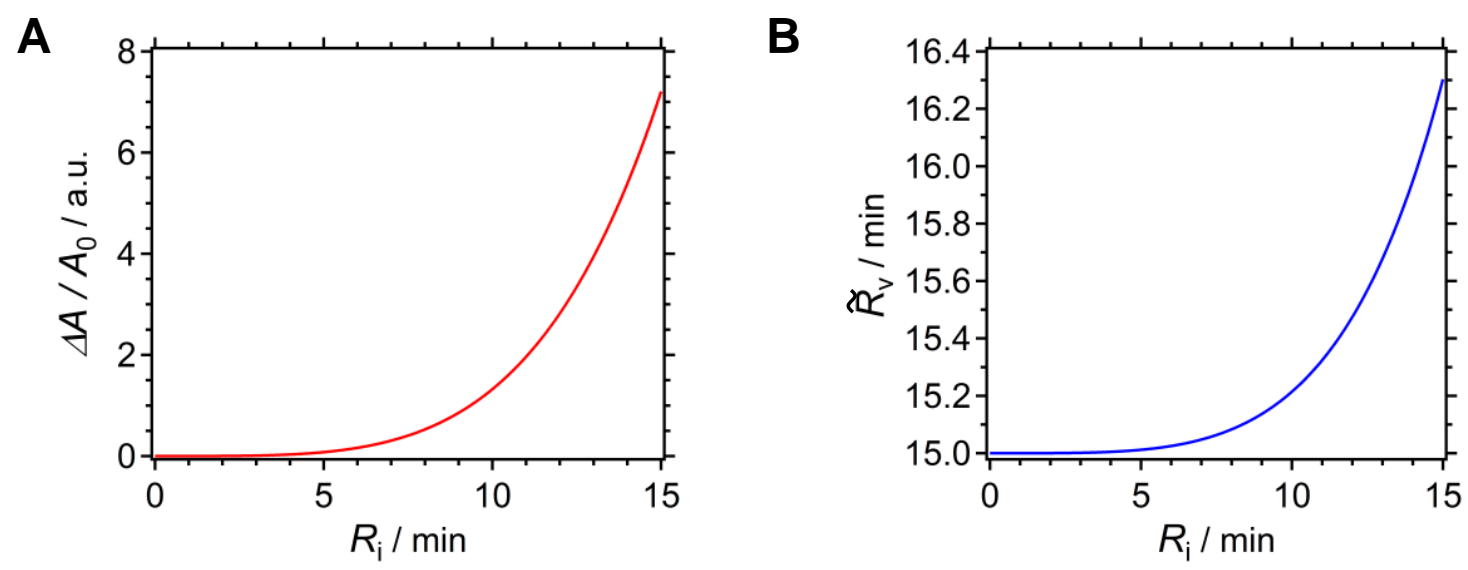

Figure 2.3. Calculated membrane area increase of an adhered GUV with increasing adhesion radius $R_{\mathrm{i}}$ (A) and increasing vesicle radius $\widetilde{R}_{\mathrm{v}}$ compared to the change in adhesion radius $R_{\mathrm{i}}(\mathrm{B})$.

The example in figure 2.3 shows the calculated area change for a GUV that was measured by confocal microscopy. The radius $\widetilde{R}_{\mathrm{v}}=15.1 \mu \mathrm{m}$ was measured and the radius $R_{\mathrm{v}}=15.0 \mu \mathrm{m}$ of the GUV was calculated with equation (11) to receive the initial radius of the freely floating GUV. In the appendix in chapter 9.1 the MATLAB script is depicted that calculated the graphs shown in figure 2.3. As a consequence, the total area change is only marginally increased by the adhesion area from $R_{\mathrm{i}}=0$ up to 
$7 \mu \mathrm{m}$ for this example. This means that the ratio of the radii $R_{\mathrm{i}} / \widetilde{R}_{\mathrm{v}}$ has to be greater than around $50 \%$ until a reasonable membrane area change is reached while the overall radius $\widetilde{R}_{\mathrm{v}}$ of the GUV changes only marginally. If the contact radius $R_{\mathrm{i}}$ becomes greater the fractional area change increases rapidly until the proposed lysis tension $(\sim 10 \mathrm{mN} / \mathrm{m})$ of the membrane which is in between $3-5 \%$ area increase compared to the relaxed surface area. In the experimental approach of this thesis it was therefore necessary to induce a large adhesion area to reach reasonable membrane tensions.

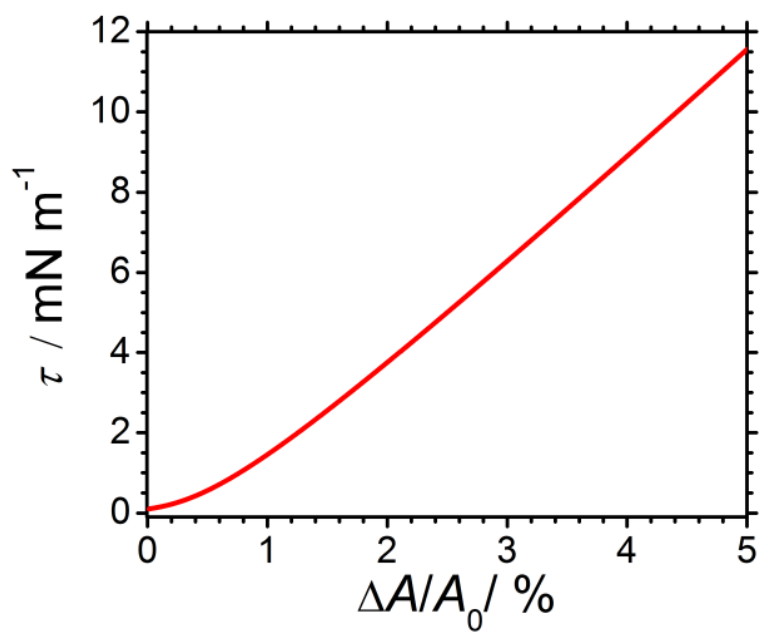

Figure 2.4. Computed tension as a function of fractional area increase using equation (3) and a prestress tension of $9.7 \times 10^{-5} \mathrm{~N} / \mathrm{m}$ that was determined by GUV-compression experiments with an AFMCantilever.

Eventually, the membrane tension can now be computed with equation (3) by knowing the bending rigidity of the membrane which is assumed to be $0.85 \times 10^{-19} \mathrm{~J} \approx 21 \mathrm{kB}_{\mathrm{B}} T, 51$ and by assuming an area compressibility modulus of $K_{\mathrm{A}}=0.28 \mathrm{~N} / \mathrm{m}$ for this lipid composition and a pre-stress tension of the adhered GUV-membrane of $\sigma_{0}=9.7 \times 10^{-5} \mathrm{~N} / \mathrm{m} \cdot{ }^{51,54}$

In figure 2.4 the calculated membrane tension as a function of fractional area change is shown. By computing the fraction area change in the first place, finally, the membrane tension for each vesicle can be generated from this calculation with equation (3). 


\section{Materials and Methods}

\subsection{Lipid vesicles}

Giant unilamellar vesicles (GUVs) and large unilamellar vesicles (LUVs) were composed of the following phospholipids: 1,2-dioleoyl-sn-glycero-3-phosphocholine (DOPC), 1,2-dioleoyl-sn-glycero-3phosphoethanolamine (DOPE), 1,2-dioleoyl-sn-glycero-3-phospho-L-serine (sodium salt) (DOPS) (all from: Avanti Polar Lipids, Alabaster, AL, USA), and 3 $\beta$-hydroxy-5-cholestene (Cholesterol) (SigmaAldrich, Taufkirchen, Germany). The lipid fluorophores Atto390®-DOPE (A390), Atto488 ${ }^{\circledR}$-DOPE (A488) and Atto594 ${ }^{\circledR}$-DOPE (A594) were purchased from ATTO-TEC GmbH (Siegen, Germany). ${ }^{66}$ Lipid films of different compositions were obtained by mixing stock solutions (1-10 mg mL-1 in chloroform), evaporation of the solvent under nitrogen flow, and removing residues of the organic solvent under vacuum at $30^{\circ} \mathrm{C}$ for $2 \mathrm{~h}$. GUVs consists out of a lipid composition resembling the natural composition of lipids in mammalian cells as used in many other vesicle fusion assays. ${ }^{67}$ 1,2-dioleoyl-sn-glycero-3phosphoethanolamine-N-(cap biotinyl) (cap-biotinyl-DOPE) was added to the lipid composition to introduce a binding interaction of GUVs membrane to avidin coated glass surfaces. Besides the lipid fluorophores A488 for the LUVs and A594 for the GUVs, the fluorescently labeled lipid dyes A390 and A594 were used to exclude a FRET effect (Förster-resonance energy transfer) when mixed in the same membrane. The FRET effect had to be considered because two fluorescently labeled lipids in the membrane can show an energy transfer from the excited fluorescently labeled lipid dye to the FRET partner which is in near proximity in the fluid membrane at around 3-6 nm at which half of the absorption energy of the donor is transferred to its FRET acceptor. ${ }^{68}$ This FRET effect was prevented by choosing lipid dyes that have a gap between emission of the excited lower wave length lipid dye and the excitation spectrum of the higher wave length lipid dye. The FRET effect can also be used as a test of SNARE mediated full membrane fusion between two vesicle fractions (see chapter 3.4.2). ${ }^{25,68-69}$

As a test of fusion between two LUV fractions containing the $\Delta N 49$-complex in the first and Syb in the second LUV fraction, the FRET-pair A488 and A594 was used which is described in chapter 3.4.2 in more detail.

In the following tables 3.1 and 3.2 the lipid compositions of GUVs, LUVs and supported lipid bilayer (SLB) for the two different experimental approaches are listed as an overview.

Table 3.1. Lipid composition of the two vesicle fractions for the experiment of adhered GUVs and fusion of LUVs.

\begin{tabular}{|c|c|c|c|c|c|c|}
\hline mol\% & DOPC & DOPE & DOPS & Cholesterol & Fluorophore & $\begin{array}{c}\text { cap-biotinyl- } \\
\text { DOPE }\end{array}$ \\
\hline GUV & 55 & 20 & 11 & 11 & 1 & 2 \\
\hline LUV & 55 & 22 & 11 & 11 & 1 & - \\
\hline
\end{tabular}


Table 3.2. Lipid composition of the supported lipid bilayer (SLB) and LUVs for experiment using the stretching device.

\begin{tabular}{|c|c|c|c|c|c|c|}
\hline mol\% & DOPC & DOPE & DOPS & Cholesterol & A390 & A594 \\
\hline SLB & 55 & 22 & 11 & 11 & 1 & - \\
\hline LUV & 55 & 22 & 11 & 11 & - & 1 \\
\hline
\end{tabular}

The figures 3.1-3.5 display the structures of the used lipids.<smiles>[2H][C@@H](COC(=O)CCCCCCCCCCCCCCCCCCCCCCCCC)COP(=O)([O-])OCC[N+](C)(C)C</smiles>

Figure 3.1. Structure of 1,2-dioleoyl-sn-glycero-3-phosphocholine (DOPC).

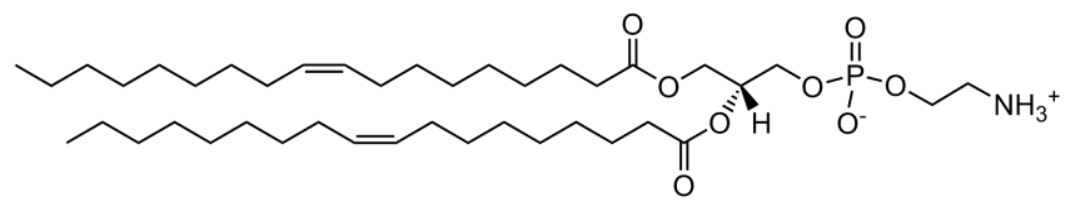

Figure 3.2. Structure of 1,2-dioleoyl-sn-glycero-3-phosphoethanolamine (DOPE).

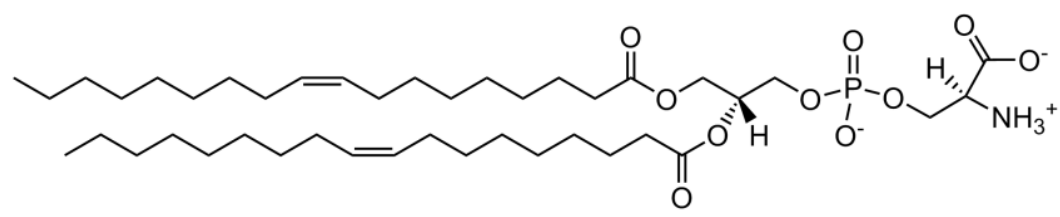

Figure 3.3. Structure of 1,2-dioleoyl-sn-glycero-3-phospho-L-serine (DOPS).<smiles>CCCCCCCC/C=C\CCCCCCCC(=O)OC[C@H](O)COP(=O)(O[NH3+])OCCNC(=O)CCCCCNC(=O)CCCC[C@H]1SC[C@@H]2NC(=O)N[C@H]21</smiles>

Figure 3.4. Structure of 1,2-dioleoyl-sn-glycero-3-phosphoethanolamine-N-(cap biotinyl) (cap-biotinylDOPE). 


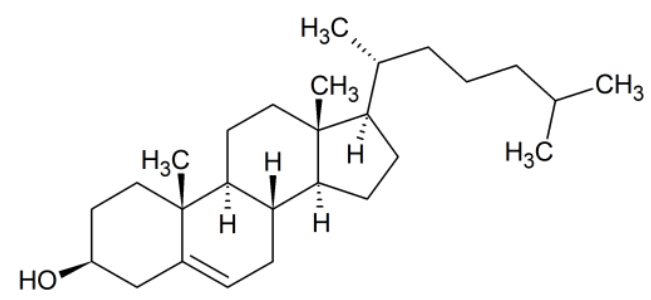

Figure 3.5. Chemical structure of $3 \beta$-hydroxy-5-cholestene (Cholesterol).

Since lipids found in nature, like 1-palmitoyl-2-oleoyl-sn-glycero-3-phosphocholine (POPC), have a high transition temperature, they were replaced by DOPC. The transition temperature from the liquid ordered phase $\left(\mathrm{L}_{0}\right)$ to the liquid disordered phase of DOPC is at $-17^{\circ} \mathrm{C}$, for DOPE at $-16{ }^{\circ} \mathrm{C}$, and for DOPS at $11^{\circ} \mathrm{C} .{ }^{70}$ Thus, experiments can be performed at room temperature with defined diffusivity of the membrane.

To get full fusion of two opposing membranes it is necessary to reduce the energy barrier. Negative spontaneous curvature of the plasma membrane of cells is essential at their fusion site. ${ }^{71}$ Behind this background the membranes for the fusion experiments contained DOPE which has a strong negative spontaneous curvature because of the small phosphoethanolamine (PE) head group. Usage of this lipid composition stabilizes a provoked hemi-fusion stalk intermediate. Thus, the fusion probability of the LUVs with the destined membrane is increased. ${ }^{71-74}$

Cells naturally also contain lipids with a phosphoserine (PS) head group which is negatively charged. SNAREs incorporated into the membrane are repelled by this charge. Their amino acid chains protrude into the cytosol above the membrane. Thereby they are easier accessible for the SNARE counterpart on the opposing membrane.

Cholesterol is an essential compound of the plasma membrane for membrane fusion at the active zones where small vesicles fuse with the plasma membrane. Clustering of SNARE proteins is found in cholesterol rich regions of the plasma-membrane. In fusion experiments, cholesterol stabilizes membranes with incorporated SNARE proteins and promotes a fast vesicle fusion. A detailed review of the influence of cholesterol on membrane fusion has been published by Yang et al. ${ }^{75-76}$ 


\subsubsection{Fluorescently labeled lipids and dyes}

In confocal fluorescence microscopy a dye molecule is excited by a LASER (light amplification by stimulated emission of radiation) with a defined wavelength. Light with a higher wavelength is emitted from the excited molecule nanoseconds after the excitation. For a deeper understanding of fluorescence microscopy the book of "Fundamentals of Light microscopy and Electronic Imaging" by D.B. Murphy provides a more detailed overview. ${ }^{77}$ Thus fluorescently labeled lipids were incorporated into the membranes of GUVs, LUVs and SLBs to make them visible with a confocal laser scanning microscope (CLSM) which is described in chapter 3.6.1 in more detail.

The lipid DOPE (Figure 3.6) labeled with the dye molecules of ATTO $^{\circledR} 390,488$ or 594 (Figure 3.7) (A390, A488, A594) were used to make the fluid membranes visible. The structure of ATTO ${ }^{\circledR 594}$ is not published but described as a carboxyl derivate similar to ATTO $^{\circledR} 488$.

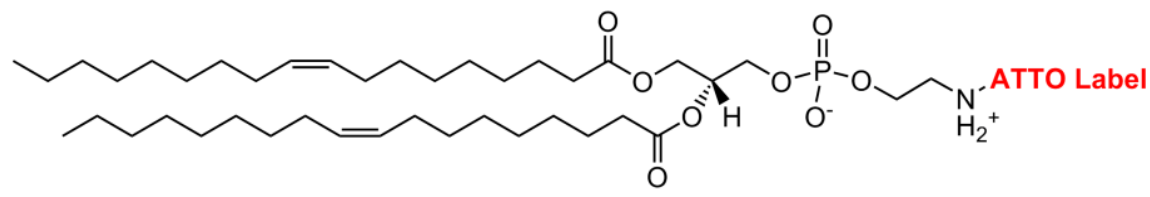

Figure 3.6. Structure of the lipid DOPE labeled with ATTO $^{\circledR} 390,488$ or 594.

A<smiles>Cc1cc(=O)oc2cc3c(cc12)C(C)CC(C)(C)N3CCCC(=O)O</smiles>

B<smiles>CN(CCCC(=O)O)C(=O)c1ccccc1C1=C2C=CC(N)=C(S(=O)(=O)O)C2OC2=C1C(=O)CCC2=[NH2+]</smiles>

C<smiles>[NH3+][OH+]S(=O)(=O)c1cc(S(=O)(=O)[O-])c2c3c1C=CC1C(S(=O)(=O)[O-])=CC(O)=C(C=C2)C31</smiles>

Figure 3.7. Chemical structure of ATTO $^{\circledR} 390(A)$ and $488(B)$ and pyranine $(C)$.

The fluorescently labeled lipid dye TexasRed ${ }^{\circledR}$ DHPE (Figure 3.8) was also used. The concentration of the fluorescently labeled lipids in the membrane was very low (1 mol\%) to ensure that these dye molecules do not disrupt the integrity and function of the membranes. The hydrophilic dyes, Pyranine (Figure $3.7 \mathrm{C}$ ) and ATTO $^{\circledR} 488$ carboxy (Figure $3.7 \mathrm{~B}$ ) were used as fluorescent markers in the LUV content to measure membrane fusion as a function of fluorescence intensity increase at the GUV content to which the LUVs could fuse. 


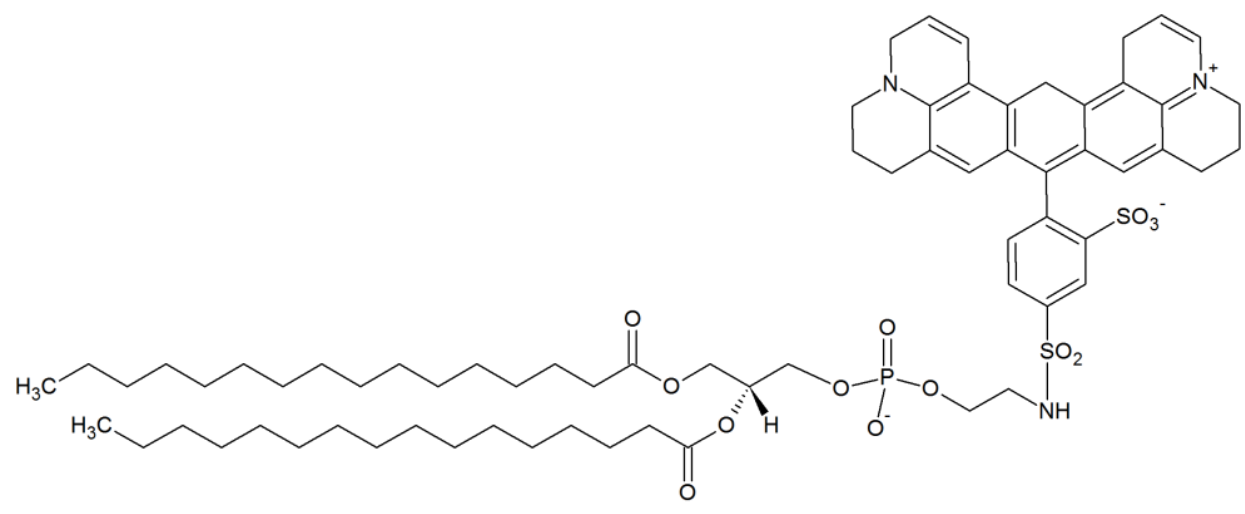

Figure 3.8. Chemical structure of TexasRed ${ }^{\circledR}$ DHPE.

A summary of the excitation and emission maxima of all used dye molecules is given in table 3.3. The fluorescently labeled lipids A488 and A594 build a FRET pair as well A390/A488, and A488/TexasRed ${ }^{\circledR}$. To keep the loss of fluorescence intensity for the dye with the smaller wavelength out A390 and A594 were mainly used.

Table 3.3. Summary of the fluorescence absorption wave length maxima $\left(\lambda_{\mathrm{abs}}\right)$, the emission wavelength maxima $\left(\lambda_{\mathrm{em}}\right)$, the extinction coefficients $\left(\varepsilon_{\max }\right)$, and fluorescence quantum yield $\left(\eta_{\mathrm{fl}}\right)$ for the fluorescent dye molecules used. . $^{78-79}$

\begin{tabular}{|c|c|c|c|c|}
\hline Dye molecule & $\boldsymbol{\lambda}_{\text {abs }} / \mathbf{n m}$ & $\boldsymbol{\lambda}_{\text {em }} / \mathbf{n m}$ & $\boldsymbol{\varepsilon}_{\max } / \mathbf{1 0}^{\mathbf{4}} \mathbf{M}^{\mathbf{- 1}} \mathbf{c m}^{-\mathbf{1}}$ & $\mathbf{\eta}_{\mathrm{fl}} / \%$ \\
\hline ATTO $^{\circledR} \mathbf{3 9 0}$ & 390 & 479 & 2.4 & 90 \\
\hline ATTO $^{\circledR} \mathbf{4 8 8}$ & 501 & 523 & 9.0 & 80 \\
\hline ATTO $^{\circledR} \mathbf{5 9 4}$ & 601 & 627 & 12 & 85 \\
\hline TexasRed $^{\circledR}$ DHPE & 582 & 600 & 11.8 & - \\
\hline
\end{tabular}




\subsubsection{Vesicles}

Lipid vesicles are common artificial membranes that are used as a simplified model of biological cell membranes and their organelles to mimic biophysical properties of vesicle membranes, especially for the investigation of membrane fusion. ${ }^{73}$ These vesicles are spherical lipid bilayers which encapsulate an aqueous buffer solution. Three different diameter size ranges are discerned for artificial vesicles which can be prepared with different methods (Figure 3.9):

1. Small unilamellar vesicles (SUVs): $d=10-100 \mathrm{~nm}$

2. Large unilamellar vesicles (LUVs): $d=100 \mathrm{~nm}-1 \mu \mathrm{m}$

3. Giant unilamellar vesicles (GUVs): $d=1-150 \mu \mathrm{m}$.

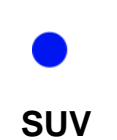

SUV

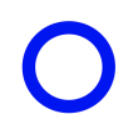

LUV

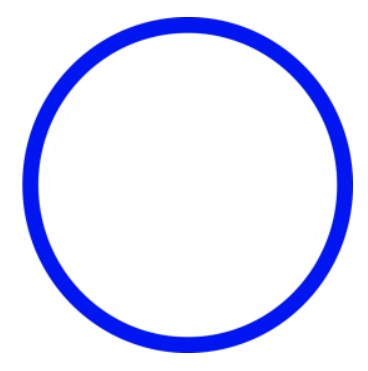

GUV

Figure 3.9. GUVs, LUVs and SUVs produced by different methods are artificial membranes that can mimic biological cells and their functions.

Dissolving a dried lipid film in a buffer results in multilamellar vesicles (MLVs) containing many stacked lipid bilayers. Different methods can be used to produce unilamellar vesicles of different sizes containing only on lipid bilayer. Generally, SUVs can be produced by dissolving a lipid film in a buffer solution and by a treatment with ultrasound. The SUVs produced by sonification can be used for spreading membranes on hydrophilic surfaces like glass or nanoporous aluminum oxide. ${ }^{80-81}$ In this study, SUVs were spread on hydrophilic PDMS surfaces. SUVs and LUVs can also be produced via the extrusion method where the buffer containing MLVs is pushed through a porous polycarbonate membrane with a defined pore size between $50 \mathrm{~nm}$ and $5 \mu \mathrm{m}$ (Lipsofast-Basic, Avestin, Ottawa, Canada). In this study LUVs were produced by detergent dilution chromatography which is described in chapter 3.4. 


\subsubsection{Electroformation of GUVs}

Giant unilamellar vesicles (GUVs) serve as a cell like structure because of their similar size to biological cells. They can be prepared by different methods, for example, by electrofromation. ${ }^{82-83}$ By applying an electric field between two electrodes consisting of platinum or indium tin oxide (ITO) that were coated with a lipid film and were placed into a aqueous solution it is possible to generate GUVs from the lipid films on the electrodes. ${ }^{54,84-85}$ Changing the conditions of the aqueous solution where the electrodes with the lipid films are placed in and applying different currents the electroformation technique is capable of producing GUVs with different sizes under many different conditions, for example high or low salinity of the used buffer, or high and low voltage applied to the electroformation chamber. ${ }^{54}$ One big challenge is the production of GUVs under physiological conditions. ${ }^{86-87}$

For all experiments in this thesis, GUVs were produced in a chamber between two ITO glass slides covered with the lipid film after the reconstitution of SNARE proteins into the LUV membranes. Drying of the LUV solution onto the ITO slides after the SNARE reconstitution (see chapter 3.4) resulted in a lipid film that covered the surface of the ITO glass slide. Between the two ITO-slides a PDMS spacer was placed so that a chamber was formed that was filled with sucrose solution with an osmolality of $150 \mathrm{mOsmol} / \mathrm{kg}$ for GUV adhesion experiments or $220 \mathrm{mOsmol} / \mathrm{kg}$ for stretching of lipid bilayers on PDMS substrate.

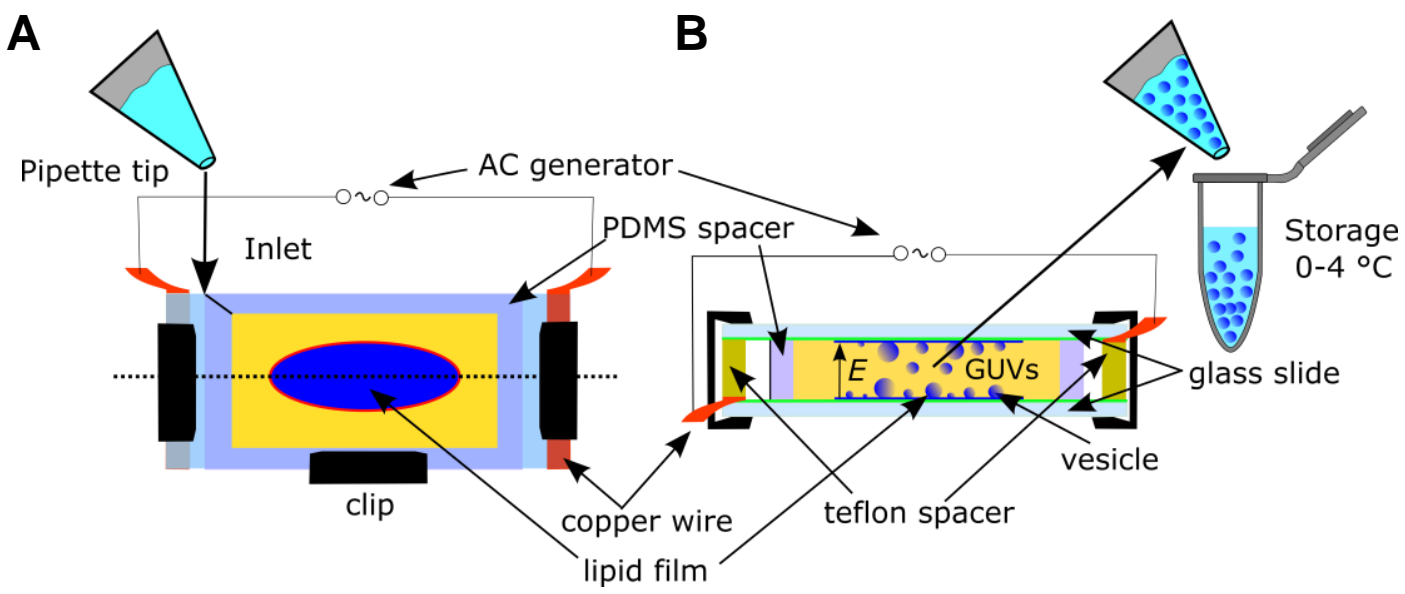

Figure 3.10. GUVs were produced in a chamber between two ITO glass slides. Both surfaces in the constructed chamber were covered with a lipid film that was dried out of a LUV solution. A) The side view of the constructed chamber shows that a PDMS spacer between the two ITO slides builds up a cavity in which the sucrose solution can be filled. A sinusoidal current was generated by the alternating current generator. B) The dashed line in (A) represents this cross-sectional view. By applying an alternating current which generates an electric field $E$ in the chamber, the lipid film swells to form GUVs.

In figure 3.10 the electroformation chamber setup is drawn that shows the two ITO slides with the PDMS spacer. The inlet at the side of the PDMS space was used to add the sucrose solution after the construction and to collect the GUV solution after the electroformation. A copper wire at the end of each ITO slide was glued on the surface. A sinusoidal voltage of $1.6 \mathrm{Vpp}_{\mathrm{pp}}$ with a frequency of $12 \mathrm{~Hz}$ was applied for 2.5-3 h with an alternating current generator (Agilent 33220A Signal Generator, Santa Clara, CA, USA) yielding GUVs with a size of 3-50 $\mu \mathrm{m}$ in diameter. After two to three hours of electroformation the GUV-sucrose solution can be removed from the chamber and stored in a tube at $0-4{ }^{\circ} \mathrm{C}$. 
The electroformed GUVs in the sucrose solution can be analyzed by confocal microscopy. Therefore, the GUVs can be adhered to a functionalized glass surface. In figure 3.11 three GUVs with different sizes $(d=9.8 \mu \mathrm{m}$ to $27.8 \mu \mathrm{m})$ were depicted exemplary in cross-sectional image.

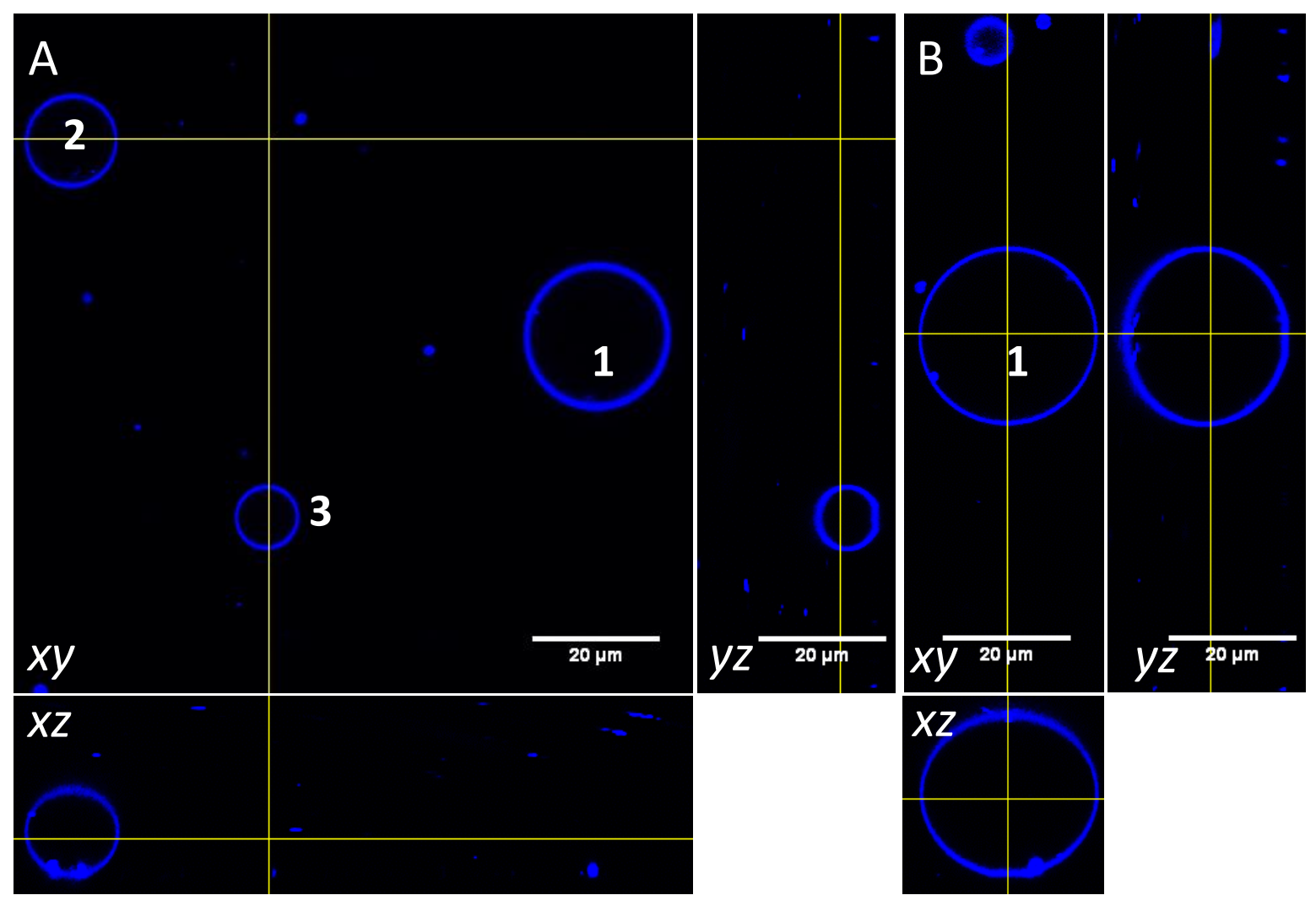

Figure 3.11. A) Three adhered GUVs produced by the electroformation method are represented in the picture which shows the cross sections $(y z, x z)$ of the drawn lines in $x y$. B) Cross-sectional view of the GUV 1. The size in diameter of the three giant vesicles range from $9.8 \mu \mathrm{m}(1)$, and $14.4 \mu \mathrm{m}$ (2) to $27.8 \mu \mathrm{m}(3)$. 


\subsection{Buffers and surface coating proteins}

The used buffer solution were essential for the fusion experiments with SNAREs because they produce a stable environment for the GUVs and LUVs with SNAREs incorporated into their membranes. The main task for the used buffers was to stabilize the hydrogen ion activity $(\mathrm{pH})$ because the zwitterionic molecule 2-[4-(2-hydroxyethyl)piperazin-1-yl]ethanesulfonic acid (HEPES) has a buffer capacity between $\mathrm{pH} 6.8$ and $\mathrm{pH} 8.6$ with an isoelectric point at $\mathrm{pH}=7.48 .{ }^{88}$ The $\mathrm{pH}$ for all buffers was set to $\mathrm{pH}=7.4$. All buffers were made with ultra-pure water from a MilliQ system (EMD-Millipore, Merck Darmstadt, Germany) filtered through a cellulose-acetate-membrane (Minisart, Sartorius, Göttingen, Germany) with a pore size of $200 \mathrm{~nm}$ and eventually degassed. The osmolality of each buffer was controlled with an osmometer (Osmomat 3000, Gonotec, Berlin, Germany). Three proteins were used to functionalize hydrophilic surfaces: Avidin from egg white (Av), Bovine Serum Albumin (BSA), and Casein from bovine milk (Cas) (Sigma-Aldrich, St. Louis, Missouri, USA). These proteins were dissolved in PBS buffer (Table 3.4): Av (1 $\mu \mathrm{M})$, BSA $(100 \mu \mathrm{M})$, Cas $(100 \mu \mathrm{M})$. Passivation of hydrophilic surfaces is a known method in literature for example of glass surfaces and micropipettes to inhibit membrane sticking to the glass or oxidized PDMS surface which is also covered with silicates. ${ }^{89-91}$

Table 3.4. Phosphate buffer composition.

\begin{tabular}{|c|c|c|c|c|}
\hline PBS & $\mathrm{NaCl}$ & $\mathrm{KCl}$ & $\mathrm{Na}_{2} \mathrm{HPO}_{4}$ & $\mathrm{KH}_{2} \mathrm{PO}_{4}$ \\
\hline Conc. $/ \mathrm{mM}$ & 137 & 3 & 10 & 2 \\
\hline
\end{tabular}

The three main buffer solutions are listed in the tables 3.5-3.7. Ethylenediaminetetraacetic acid (EDTA) was added to the HEP220 and HEP3 buffer because membrane fusion in biological cells depend on the flux of calcium ions and to show that membrane tension is responsible for increased LUV fusion efficiency EDTA was added to the used sample buffers to prevent fusion induced only by residual calcium ions. ${ }^{10}$ Dithiothreitol (DTT) is a common redox reagent that reduces disulfide bonds to thiols by a two sequential thiol-disulfide exchange reactions. DTT was added to all buffers for samples containing GUVs or LUVs with SNAREs to prevent clustering of the SNARE proteins by disulfide bridges. The buffers HEP150 and Mg150 were used for the GUV adhesion experiment on avidin functionalized glass surfaces. HEP150 and Mg150 had the same osmolality to prevent osmotic pressured between the content and surrounding solution of GUVs. The buffers HEP220 and HEP3 were used for the experiments of dilated PDMS with supported lipid bilayers. HEP3 in table 3.8, was used as a hyperosmotic buffer to induce spreading of GUVs on the hydrophilic PDMS surface to form defined areas of membrane patches. 
Table 3.5. The HEP150-buffer was used as the working buffer in all vesicle fusion experiments with adhered GUVs for LUV incubation.

\begin{tabular}{|c|c|c|c|c|}
\hline HEP150 & HEPES & $\mathrm{KCl}$ & DTT & $\mathrm{mOsmol} \mathrm{kg}^{-1}$ \\
\hline Conc. $/ \mathrm{mM}$ & 15 & 67.5 & 0.1 & $150 \pm 2$ \\
\hline
\end{tabular}

Table 3.6. The Mg150-buffer was added to a solution of HEP150 to increase the concentration of divalent ions and to increase the adhesion area of GUVs on the functionalized glass surface.

\begin{tabular}{|c|c|c|c|c|c|}
\hline Mg150 & HEPES & KCl & DTT & $\mathrm{MgCl}_{2}$ & $\mathrm{mOsmol} \mathrm{kg}^{-1}$ \\
\hline Conc. $/ \mathrm{mM}$ & 15 & 52.5 & 0.1 & 10.0 & $150 \pm 2$ \\
\hline
\end{tabular}

Table 3.7. The HEP220-buffer was used as the working buffer in all vesicle fusion experiments on SLBs for LUV incubation.

\begin{tabular}{|c|c|c|c|c|c|}
\hline HEP220 & HEPES & KCl & EDTA & DTT & $\mathrm{mOsmol} \mathrm{kg}^{-1}$ \\
\hline Conc. $/ \mathrm{mM}$ & 20 & 98.5 & 1.0 & 0.1 & $150 \pm 2$ \\
\hline
\end{tabular}

Table 3.8. The HEP3-buffer was used to increase the concentration of salts in the buffer surrounding the GUVs to produce a hyperosmotic pressure to the GUVs for spreading them on the surface.

\begin{tabular}{|c|c|c|c|}
\hline HEP3 & HEPES & $\mathrm{NaCl}$ & EDTA \\
\hline Conc. $/ \mathrm{mM}$ & 20 & 300 & 1.0 \\
\hline
\end{tabular}




\subsection{SNARE proteins and purification ${ }^{2}$}

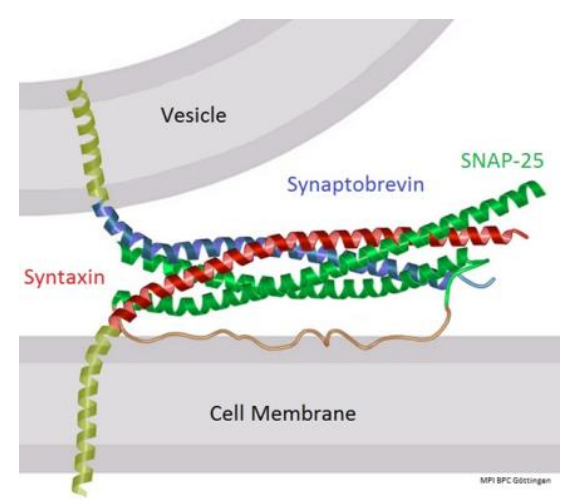

Figure 3.12. Illustration of SNARE proteins that form the zippered complex. The core complex of SNAREs contains syntaxin 1A, SNAP-25, and synaptobrevin-2.

The purification of the two SNARE-complexes ( $\triangle N 49$, Syb 2) was done by Partho Halder from the group of Reinhard Jahn (Max-Planck-Institute for Biophysical Chemistry, Göttingen) as described below.

The N-ethylmaleimide-sensitive-factor attachment receptor (SNAREs) were used in the lipid bilayers as the fusion core complex to merge two opposing lipid bilayer. The fusion core complex of SNAREs is composed of the $\Delta N 49$-complex $(\Delta N 49)(m=40.963 \mathrm{kDa})$ and synaptobrevin-2 (1-116) (Syb 2) $(m=12.691 \mathrm{kDa})$. The $\Delta \mathrm{N} 49$-complex is formed by mixing syntaxin1A (183-288), SNAP-25 (1-206) and Syb2 (49-96) in a molar ratio of 1:1:1.5 and purified as described earlier. ${ }^{9}, 92-95$

The image in figure 3.12 illustrates the zippering of the four bundle $\alpha$-helices of the SNARE core complex that brings the opposing vesicle membrane into close contact with the cell membrane is provided by the Max-Planck-Institute for Biophysical Chemistry Göttingen and relates to the crystal structure by Sutton et al. ${ }^{8}$

Proteins were overexpressed in E. coli BL21 (DE3) with a N-terminal His6-tag using the pET-vector (Novagen) and affinity-purified using $\mathrm{Ni}^{2+}$-nitrilotriacetic acid (NTA) agarose (Qiagen) resin followed by thrombin cleavage to remove the His6-tags. The proteins were further purified by ion-exchange chromatography using the ÄKTA system (GE Healthcare, Chicago, USA). Full-length synaptobrevin (1116) (Syb2) was purified as described by Pobbati et al.. ${ }^{96}$ Purified proteins were snap-frozen with liquid nitrogen and stored at $-80^{\circ} \mathrm{C}$.

2 Performed by Parhto Halder (Max-Planck-Institute for Biophysical Chemistry, Göttingen) 


\subsection{Reconstitution of SNAREs}

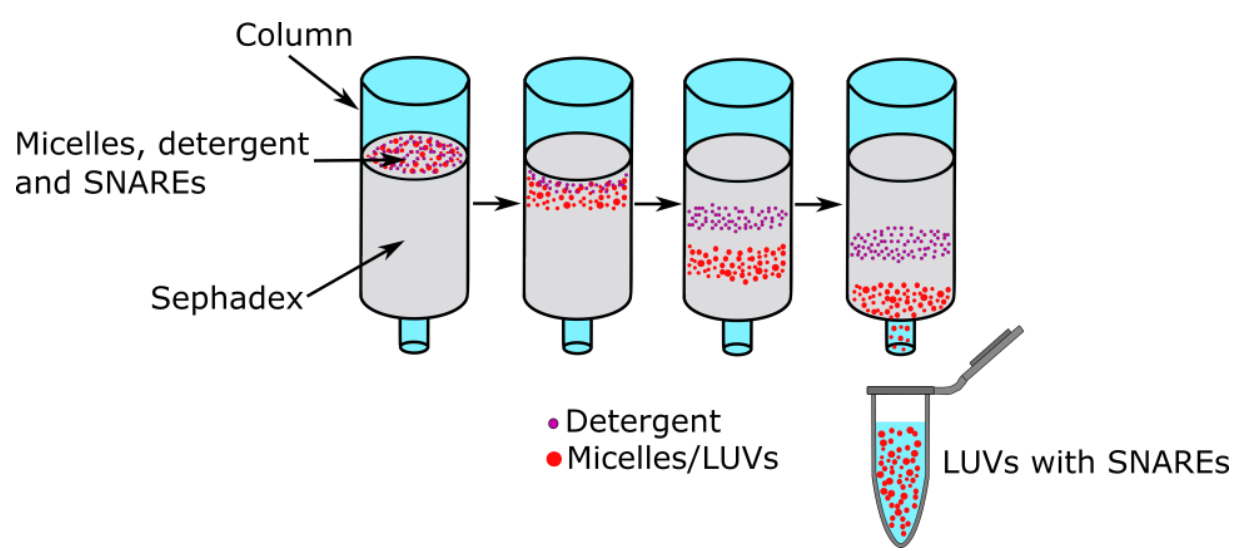

Figure 3.13. Scheme of SNARE-reconstitution into the vesicle membranes by the detergent dilution method. The detergent solution containing the lipids and SNARE proteins was given onto the Sephadex column. Elution with buffer HEP150/HEP220 results in a LUV solution that was collected in a reaction tube. While elution of the detergent, the SNARE proteins incorporate into the lipid bilayer of the LUVs by inserting their trans membrane sequence in the amino acid chain.

The reconstitution of the synaptobrevin or the $\Delta N 49$-complex into lipid vesicles was performed as previously described by Schwenen et al..24 Therefore lipid films $(630 \mathrm{nmol})$ were dissolved in HEP150/HEP220-buffer ( $50 \mu \mathrm{L}$ ) containing n-octyl- $\beta$-D-glucopyranoside (NOG) (100 mM) and incubated for 30 minutes to receive a solution of micelles. Both stock solutions of the proteins contained $1 \% 3-[(3-$ Chloamidopropyl)dimethylammonio]-1-propanesulfonate (CHAPS). The detergent molecule were added to the lipids in a small buffer solution to generate micelles. The critical micelle concentration (CMC) has to be considered as a crucial value for the use of detergents. At this critical concentration either detergent molecules form micelles as well as the lipids in the solution with the detergent are dissolved to form mixed micelles. ${ }^{97}$ The micelles and proteins were mixed and incubated for 30 minutes on ice. GUVs were prepared with the $\triangle N 49$-complex (2 nM), LUVs with synaptobrevin (2 nM). To remove the detergent molecules a Sephadex column (illustra NAP-25, GE Healthcare) was prepared with HEP150/HEP220-buffer (Figure 3.13). After the elution of the micelle-protein mixture the resulting SNARE protein containing LUVs $(50-900 \mathrm{~nm}$ in diameter) were collected in a reaction tube. The size distribution of the LUVs was measured by dynamic light scattering (chapter 3.4.1). Concentrating the vesicle solution in a vacuum centrifuge (Concentrator 5301, Eppendorf, Hamburg) to a volume of 80 to $150 \mu \mathrm{L}$ and elution in a column with pure water results in an ion free vesicle solution that was again concentrated to a final volume of approximately $100 \mu \mathrm{L}$. The vesicles solution with synaptobrevin was given into a small reaction tube and dried in a desiccator filled with a saturated sodium chloride solution. LUVs were obtained by dissolving the lipid film in the reaction tube for 30 minutes with HEP150/HEP220buffer. Small droplets $(2 \mu \mathrm{L})$ with LUVs containing the $\Delta$ N49-complex were given onto ITO-slides and dried in the desiccator. With the electroformation technique GUVs were produced (chapter3.1.3). The chamber inside was filled with sucrose solution (150 mOsmol/ $/ \mathrm{kg}$ ) and a sinusoidal voltage of $1.6 \mathrm{Vpp}$ with a frequency of $12 \mathrm{~Hz}$ was applied for 2.5-3 $\mathrm{h}$ to yield GUVs with a size of about 3-50 $\mu \mathrm{m}$ in diameter. The GUV-and LUV-solution can be stored on ice for a maximum of one day. The size distribution of the LUVs diameter was determined by dynamic light scattering. 


\subsubsection{Dynamic light scattering - LUV size distribution}

Dynamic light scattering (DLS) is a common technique to determine the size distribution of particles in hydrodynamic systems.98-99 After the reconstitution of SNAREs with the detergent dilution chromatography the vesicle size distribution of the LUVs was determined by DLS. DLS measurements were performed using Zetasizer Nano S. A small volume of the LUV solution (100-200 $\mu \mathrm{L}$ ) was given into a cuvette. Then the measurement was performed after an equilibration time of 120 seconds. Three measurements using the same sample were done and the resulting data averaged.

The measurement of the LUV size distribution is shown in figure 3.14. LUVs for that measurement went through the detergent dilution chromatography two times as described in chapter 3.4. The extrusion of the LUV solution was done with a $1 \mu \mathrm{m}$ porous membrane to remove larger dust particles that could disturb the DLS measurement. Figure 3.14 shows that the LUV size distribution has a maximal intensity at around $200 \mathrm{~nm}$ in diameter. The size distribution of LUVs ranges from $50 \mathrm{~nm}$ up to $1 \mu \mathrm{m}$ in diameter. The measured hydrodynamic diameter is assumed to represent the actual diameter of the LUVs. Small LUVs with a diameter $<300 \mathrm{~nm}$ cannot be detected with a confocal microscope because of ABBE's resolution criterion but larger LUVs $(>300 \mathrm{~nm})$ can. This DLS measurement of LUVs serves as a fundamental information for the membrane fusion experiments to compare the size and volume of the GUVs and LUVs.

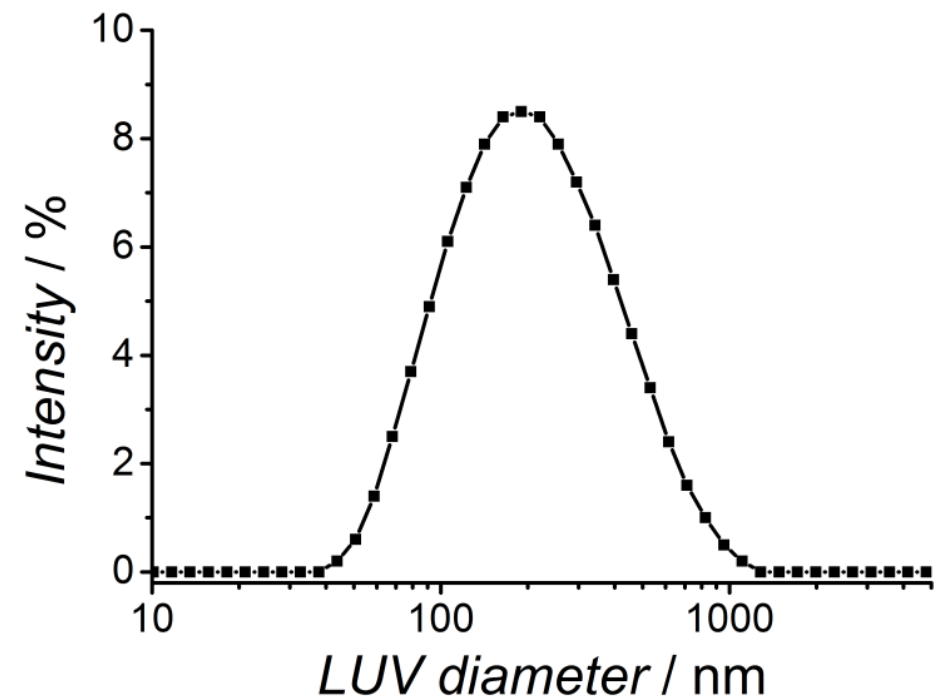

Figure 3.14. Measured size distribution of LUVs after the detergent dilution chromatography. LUV diameter is shown on a logarithmic scale. 


\subsubsection{Vesicle Fusion assay after protein reconstitution}

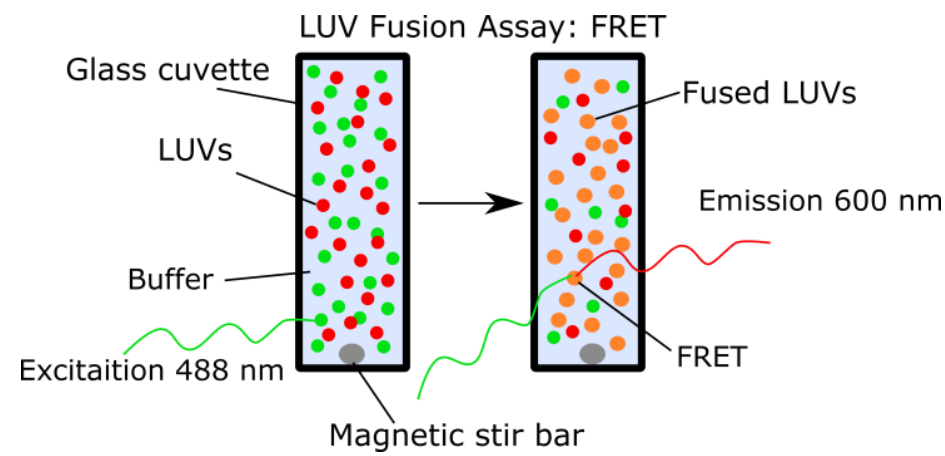

Figure 3.15. Scheme of the vesicle fusion assay used to test the successful reconstitution of SNAREs into the LUV membranes.

With the vesicle fusion assay, illustrated in figure 3.15 , the reconstitution of the $\Delta N 49$-complex and the Syb was proved by mixing two fractions of LUVs each with one of the SNAREs and a fluorescently labeled lipid that was the corresponding FRET pair. LUVs were obtained after the detergent dilution to reconstitute the SNARE proteins into the membranes. At first, the LUV solution containing the dye A594 $(50 \mu \mathrm{L})$ and the $\Delta$ N49-complex was added into a glass cuvette (108F QS, Hellma Analytics, Müllheim, Germany) with HEP220-buffer $(800 \mu \mathrm{L})$. The second LUV fraction $(50 \mu \mathrm{L})$ containing A488 and Syb2 was added to the buffer solution with the first LUV fraction and stirred for 45 minutes. While the addition of the two LUV solutions and stirring of the buffer solution the fluorescence intensity of A594 was measured at a wavelength of $627 \mathrm{~nm}$ with a fluorimeter (FluoroMax, Horiba, Kyoto, Japan). For this purpose an excitation lamp with a wavelength of $501 \mathrm{~nm}$ was used to excite the lipid dye A488 in the LUV membranes. Via the FRET-effect A594 shows a fluorescence if the membranes between the two LUV fractions fuse with each other. An increase in fluorescence intensity of A594 indicates LUV fusion between the LUV fractions with the SNARE proteins.

In figure 3.16 the fluorescence intensity of A594 at a wavelength of $627 \mathrm{~nm}$ is drawn in the graph. The red curve for LUVs containing SNAREs shows an increase in fluorescence intensity after the addition of the second LUV fraction containing A488 and Syb due to the fusion of the LUVs. As a reference, the blue curve in the graph, representing LUVs without SNAREs, shows only a slightly fluorescence intensity increase. With this vesicle fusion assay it was proven that fusion of membranes, LUV respectively, can be achieved with the used lipid composition and the reconstituted SNARE proteins. 


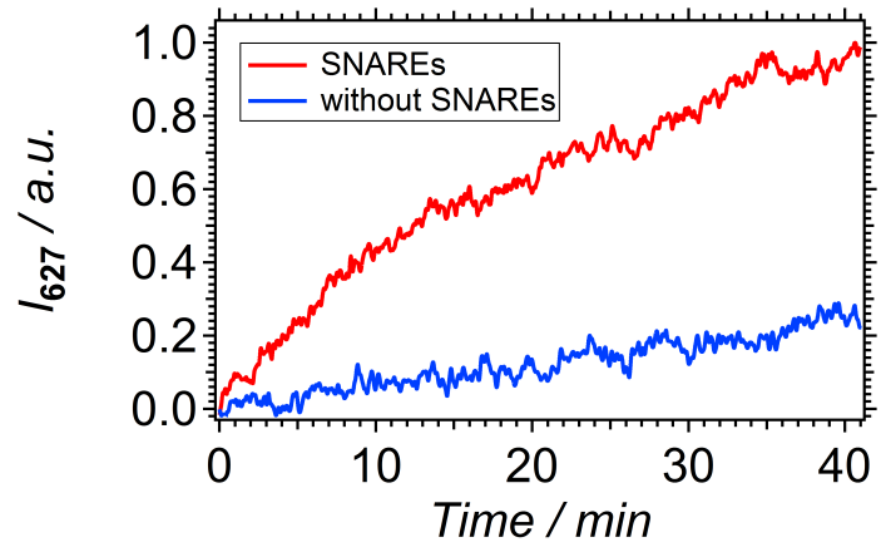

Figure 3.16. The vesicle fusion assay showed that membrane fusion occurs after the addition of the second LUV fraction (red) compared to LUVs without SNAREs (blue). 


\subsection{Polydimethylsiloxane (PDMS)}

Dilatation of planar membrane patches on a surface can be achieved by applying a biaxial force. The elastic and biocompatible Polydimethylsiloxane (PDMS) was used for a membrane stretcher device. PDMS is a common material that is used as substrate for cellular adhesion, motility or to spread lipid bilayers onto. ${ }^{58,61,100}$ PDMS is colorless, transparent, inert and a non-toxic polymer with a YouNG's modulus between $50 \mathrm{kPa}$ and $4 \mathrm{MPa}$ depending on the curing time scale and temperature. ${ }^{100}$

\subsubsection{Synthesis of PDMS}

For all membrane stretcher devices the two components kit Sylgard ${ }^{\mathrm{TM}} 184$ (Dow Corning, Midland, MI, USA) was used to synthesize all PDMS parts. Sylgard ${ }^{\mathrm{TM}} 184$ consists of a prepolymer (base) and a curing agent which were mixed with a weight ratio $(\mathrm{w} / \mathrm{w})$ of 10:1. The base serves as a vinyl terminated PDMS-macro-monomer $\left(\mathrm{R}^{1} \mathrm{CH}_{3} \mathrm{SiCH}=\mathrm{CH}_{2}\right)$ (Figure 3.17) which reacts via the platinum-complex catalyzed hydro-silylation with the cross-linking agent, a hydrosilane (Si-H).<smiles>[R]O[Si](C)(C)C=C</smiles>

Macro-polymer<smiles>[R]O[Si](C)(C)C=C</smiles>

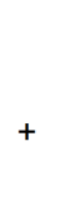

$+$<smiles>[R]O[Si](C)(C)O[Si](C)(C)O</smiles><smiles>[R]O[SiH](C)O[Si](C)(C)O</smiles>

Cross-linking agent

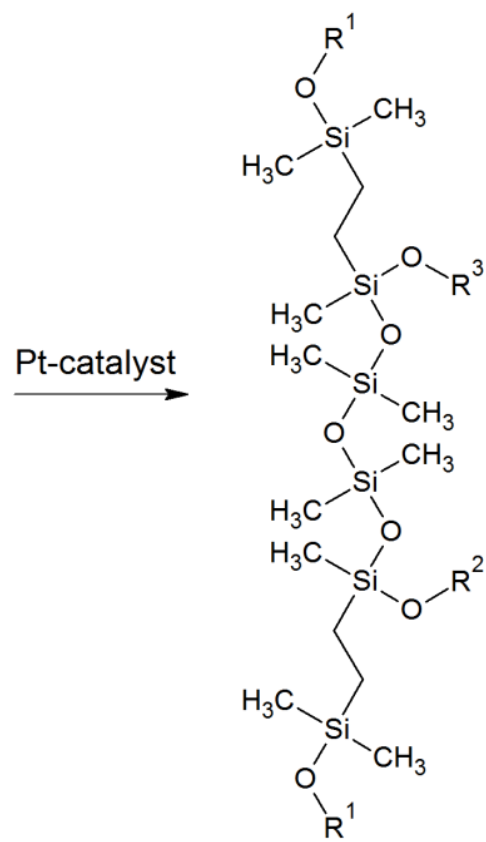

Figure 3.17. Mechanism for the synthesis of PDMS with the Sylgard ${ }^{\mathrm{TM}} 184$ kit. $^{101}$

The base and curing agent mixture $(3.0 \mathrm{~g} / 0.3 \mathrm{~g})$ were degassed under vacuum for 30 minutes to receive a colourless and high viscous fluid. Defined structures of the PDMS were produced as follows with molding and spin coating techniques with the mixed and degassed agents. 


\subsubsection{Design and fabrication of membrane stretcher device ${ }^{3}$}

The membrane stretching device (Figure 3.18) was developed and designed by Laura Turco and Marco Tarantola (Max Planck Institute for Dynamics and Self-Organization, Göttingen, Germany). The device consists out of a stretchable PDMS membrane on top of a milli-structured layer. The PDMS layer has one central channel $\left(3 \times 1 \times 20 \mathrm{~mm}^{3}\right)$ and two side channels $\left(1 \times 1 \times 16 \mathrm{~mm}^{3}\right)$. By applying negative pressure in the side channels, the walls deform the membrane sheet in the central channel (Figure $3.18 \mathrm{C}$ ). The thickness of the layer and the membrane sheet is about $5 \mathrm{~mm}$ and $180 \mu \mathrm{m}$, respectively. The membrane stretcher design was inspired by a similar device developed by Hu et al.,61 and was produced by replica molding. ${ }^{102}$ The PMMA (poly-methyl methacrylate) mold master was fabricated with a CNC milling machine (DMC 1035, DECKEL MAHO, Bielefeld, Germany). The PDMS prepolymer was mixed with curing reagent $(10: 1 \mathrm{w} / \mathrm{r})$, poured over the mold master, placed in a vacuum chamber for degassing and heated to at $75{ }^{\circ} \mathrm{C}$ for $45 \mathrm{~min}$. The solidified PDMS layer was peeled off from the master and bounded subsequently to the PDMS membrane sheet.

A

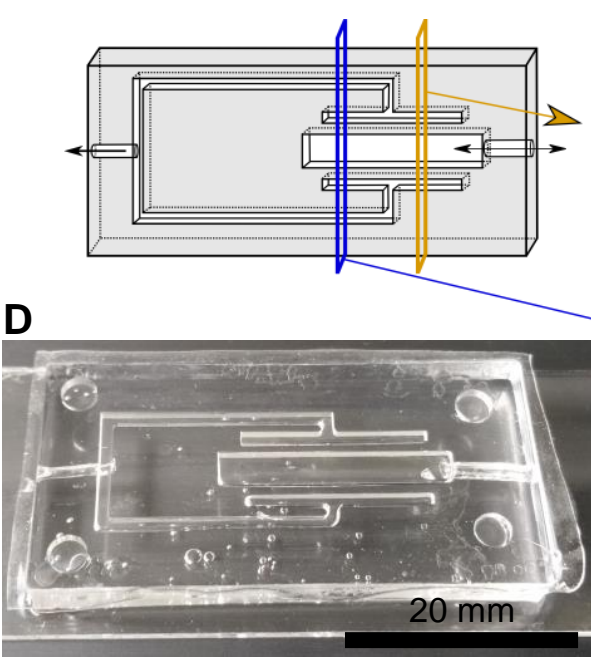

B

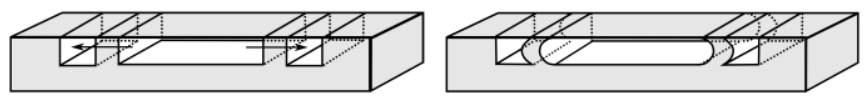
E

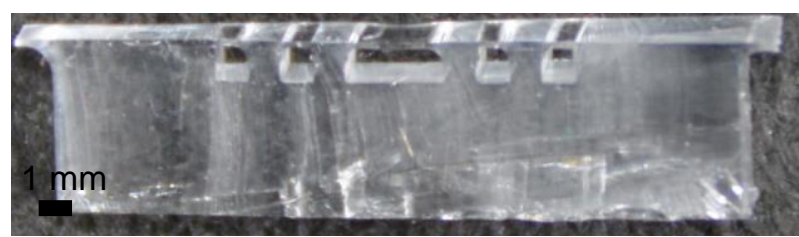

Figure 3.18. Schematic representation of the membrane stretcher device. A) Drawing of the membrane stretcher device with cuts for the cross-sectional views. B) Cross sectional view of the device that shows the two side channels to the main chamber. The thin PDMS layer is unstretched. C) A reduction of the air pressure in the side channels results in a stretch of the thin PDMS sheet. D) The photo depicts the successful constructed PDMS membrane stretcher device. E) A cross sectional image of the PDMS stretching device shows the two channels and the connected channel system.

To produce the membrane sheet the degassed base and curing agent mixture $(3.0 \mathrm{~g} / 0.3 \mathrm{~g})$ was given onto the silicon wafer functionalized with Cyclo-octafluorobutane $\left(\mathrm{C}_{4} \mathrm{~F}_{8}\right)$ (Caesar, Jülich, Germany) that was placed on a spin coating system (SCS G 3 Spin Coater Series, Special Coating Systems, Indianapolis, IN, USA). The functionalization of the silicon wafer surface with $\mathrm{C}_{4} \mathrm{~F}_{8}$ served as a passivation to inhibit the formation of covalent bonds between the PDMS and the silicon atoms on the wafer surface. For the production of a very thin PDMS layer the spin coating system rotated the silicon

${ }^{3}$ Developed by Laura Turco and Marco Tarantola (Max Planck Institute for Dynamics and SelfOrganization, Göttingen, Germany). 
wafer with $500 \mathrm{rpm}$ for 30 seconds with a starting and ending ramp of ten seconds. Then the wafer with the fluid was given into an oven set to $75^{\circ} \mathrm{C}$ for 45 minutes to yield a very thin, polymerized PDMS membrane sheet on the stretching device. The thickness of these layers was $180 \mu \mathrm{m}$ (Figure 3.19) and has been measured with the bright field microscope BX51 (Olympus, Tokio, Japan) and a CCD-camera (DP71, Olympus). Subsequently, the previously prepared milli-structured layer was placed on a wafer with a fluid mixture of the base and curing agent for a few seconds. Then it was placed on the wafer with the cured, thin PDMS layer. Again the wafer was given into the oven at $70{ }^{\circ} \mathrm{C}$ for 30 minutes (Figure $3.19 \mathrm{~B}$ ). This procedure leads to a solid connection between the thin PDMS layer and the PDMS chamber part. The thin PDMS sheet of the substrate was cut out yielding a smooth surface on top of the thin PDMS sheet. Due to its soft and flexible behavior the thin PDMS sheet could be stretched up to a few percent of its initial area until ruptures occur. Naturally, the extension of the PDMS surface is anisotropic. ${ }^{61}$ In the middle of the sheet between the side chamber walls the area dilatation is close to zero but increases towards the side wall, as previously visualized by $\mathrm{HUH}$ et al. using quantum dots sticking to the PDMS surface. ${ }^{61}$ Here, the anisotropy is an advantage since it allowed us to generate a broad spectrum of different membrane area changes within one sample. 
A

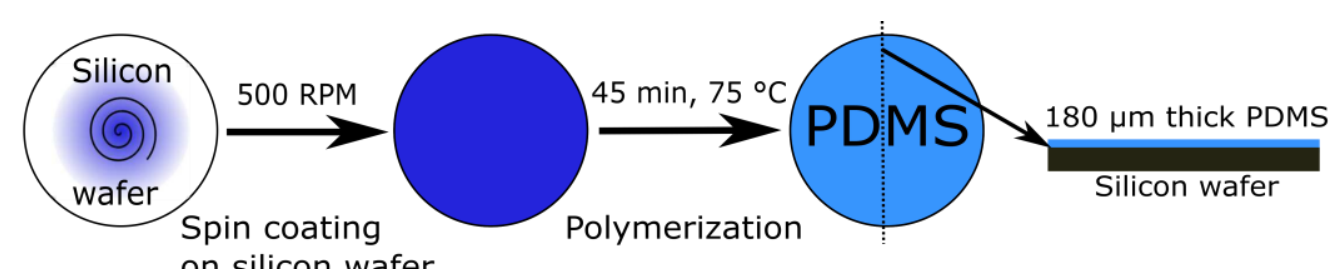
on silicon wafer

polymerized

fluid mixture base / curing agent

B

Layer surface coated with base and curing agent
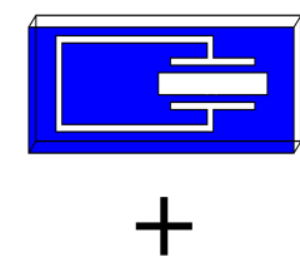

PDMS on Si wafer
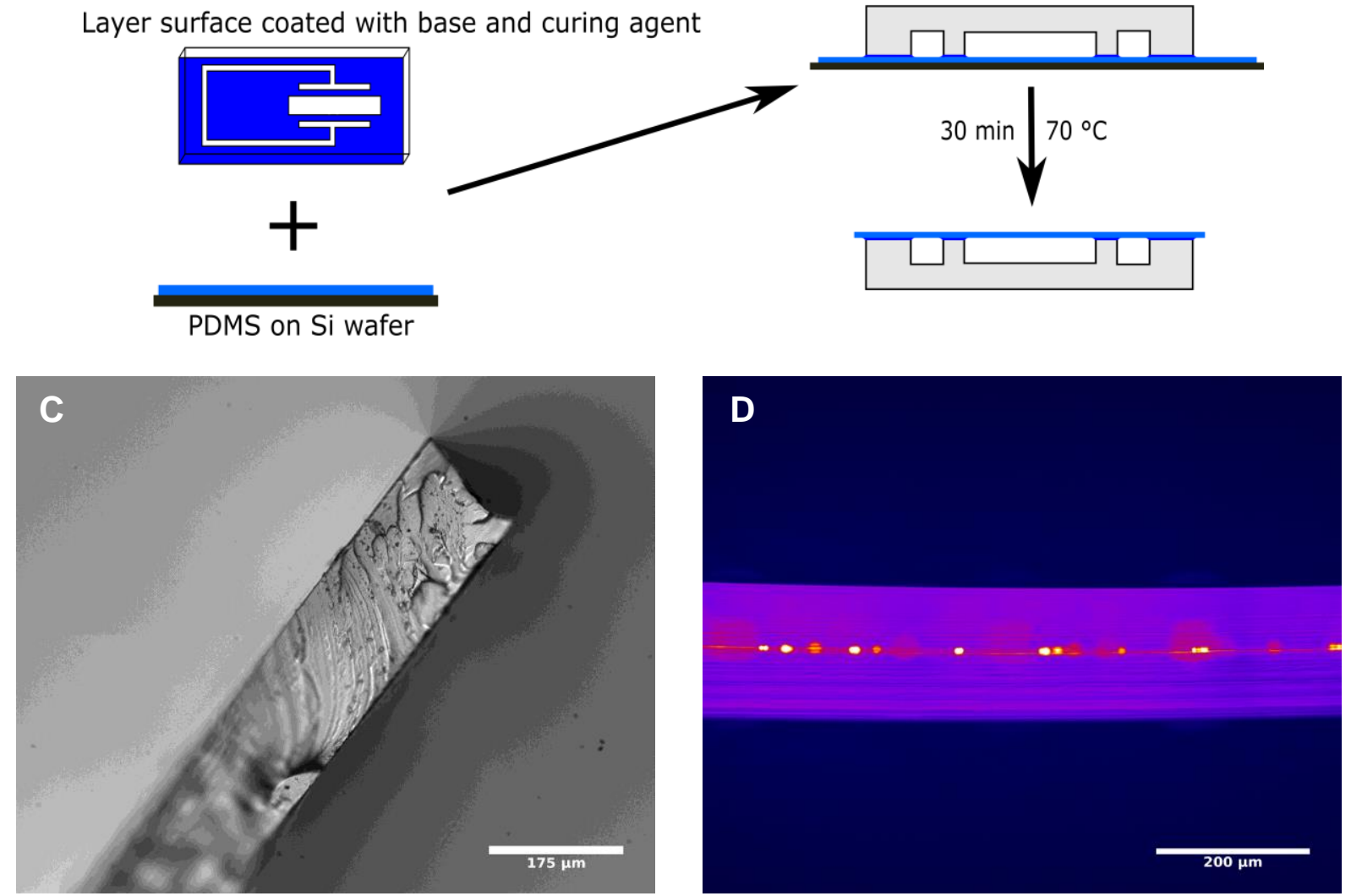

Figure 3.19. Schematic illustration of the fabrication of the membrane stretcher device. A) Spin-coating of base and curing agent mixture on a silicon wafer functionalized with $\mathrm{C}_{4} \mathrm{~F}_{8}$ led to a wafer surface fully covered with the prepolymer reactants which then polymerize $75^{\circ} \mathrm{C}$ for 45 minutes. B) The PDMS layer was dipped onto a silicon wafer with a thin, spincoated layer of the prepolymer reactants (blue), quickly removed, and given onto the cured PDMS of the silicon wafer. The prepolymer connects the thin, spincoated PDMS layer with the PDMS layer containing the channel system. C) The PDMS layer thickness of around $180 \mu \mathrm{m}$ was measured with bright field microscopy. D) A cross-sectional view of a PDMS sheet that was spincoated two times with 950 RPM. In the middle of the layer, fluorescent beads were incorporated to test the extendibility of the PDMS sheet. 


\subsection{Fluorescence microscopy}

In fluorescence microscopy the spontaneous light emission of a molecule is measured which is a result of a transition of an electron from a higher energy state into its initial ground state. The collapse of the molecules high energy state is responsible for the release of a photon which has a longer wavelength compared to the absorbed photon for the excitation of the molecule (Stokes-shift). ${ }^{103}$ The absorption of a photon is very fast $\left(10^{-15} \mathrm{~s}\right)$. An average lifetime of $10^{-8} \mathrm{~s}$ in the excited state the photon is released. For the transition of the electron from the ground state to the electronically excited state, it is most likely that the transition occurs vertical without changes in the position of the nuclei which is called the FRANCKCONDON principle. ${ }^{77,103}$ The books "Fundamentals of Light Microscopy and Electronic Imaging" by MURPHY and "Principles of Fluorescence Spectroscopy" by LAKOWICZ give a more detailed overview to this method. ${ }^{77,104}$

Fluorescence microscopy was the main technique that was used in the experiments for this thesis, especially confocal LASER scanning microscopy (CLSM) (figure 3.20). This method enables to gather information about the geometry of adhered giant vesicles and the area change of membrane patches on a dilated PDMS surface. CLSM can be used as an imaging technique for the investigation of LUV fusion on pore spanning membranes and for the measurement of the three dimensional geometry of adhered GUVs to obtain the membrane tension of the GUVs. ${ }^{24,49}$ On the one hand, in this thesis CLSM was used to measure z-stacks of GUVs to receive a three dimensional image necessary for membrane tension calculation. On the other hand, CLSM was used for the measurement of membrane areas on dilated PDMS surfaces. The used fluorescent markers were fluorescently labeled lipid dyes that are described in chapter 3.1.1. Confocal LASER scanning microscopy

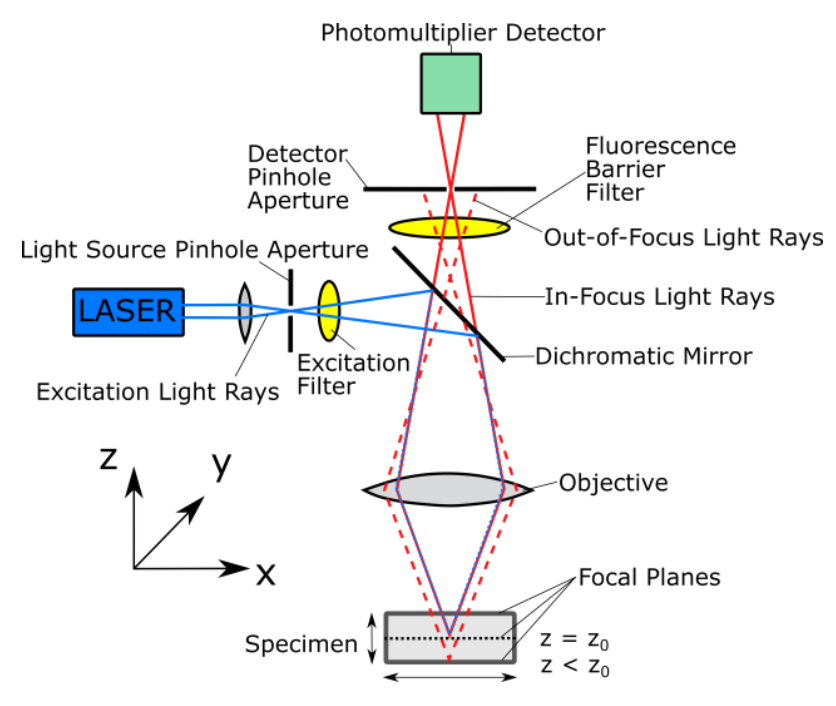

Figure 3.20. Drawing of a CLSM setup.

Confocal microscopy experiments were performed with the BX61 and a FV1200 CLSM unit (Olympus, Tokio, Japan) with a $60 \times$ water objective (Olympus). In figure 3.20 the setup of a confocal microscope is drawn schematically. LASERs with a wavelength of $405 \mathrm{~nm}, 488 \mathrm{~nm}$ and $561 \mathrm{~nm}$ were used in a nonsequential mode with a scanning rate of $2 \mu$ ser pixel for each LASER. The pixel size varied but was usually kept small to reach the resolution limit of the setup. 


\subsubsection{Image stacks of GUVs}

Three-dimensional (3D) images were taken by changing the focal plane in $z$-axis (Figure 3.21) of the specimen. The collected images with a slightly moved focal plane can be stacked to generate a threedimensional view of the adhered GUVs. Changing the focal plane was done automatically by the CLSM software on the microscope. However, the setup of the CLSM did change the focal plan in $z$-axis

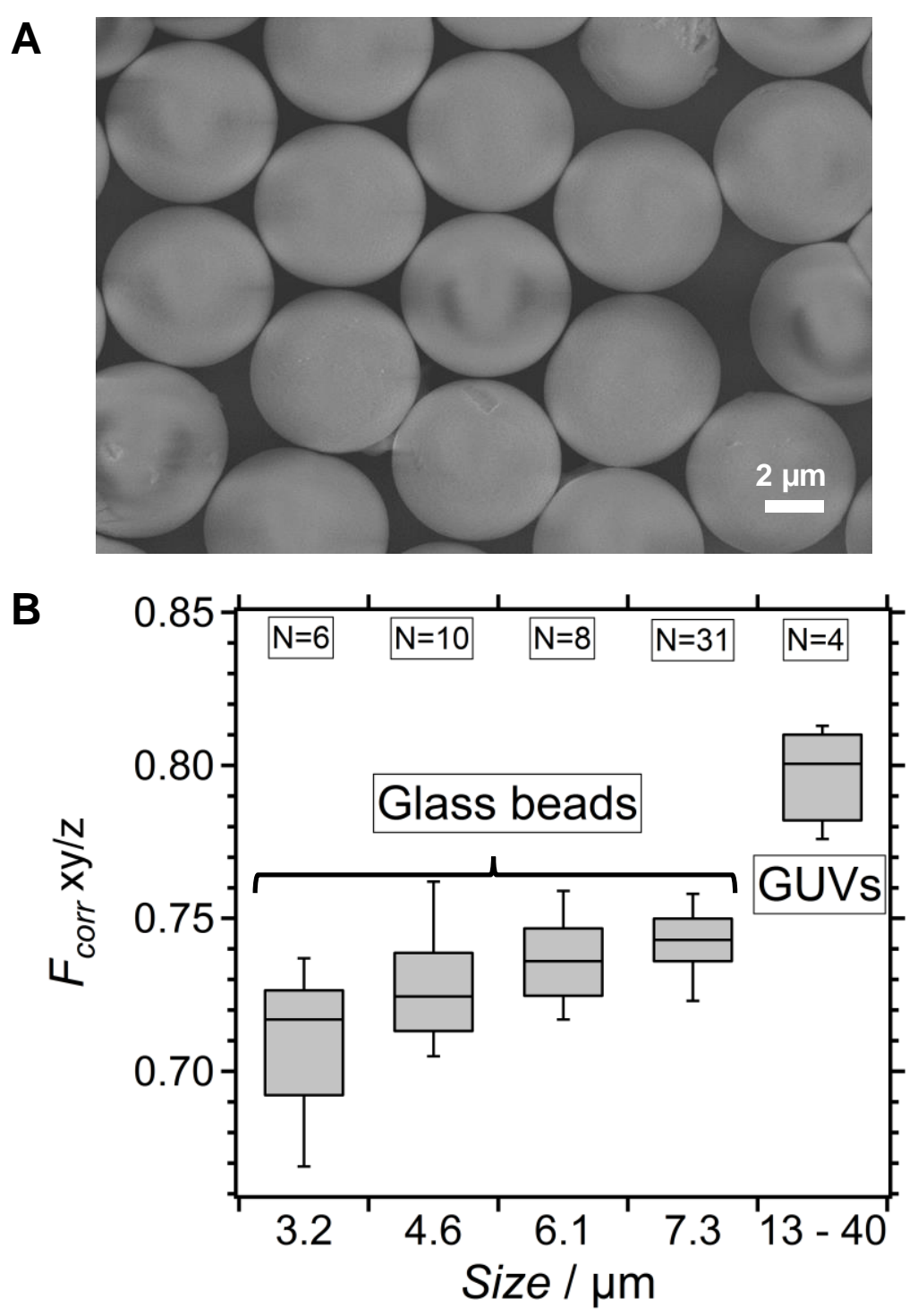

Figure 3.21. A) Scanning electron microscopy image of monodispersed glass beads with an average size of $6.5 \mu \mathrm{m}$ in diameter. B) Correction factor for 3D images measured with the CLSM from image stacks of membrane coated glass beads and slightly adhered GUVs. Boxes (25\%-75\%), whiskers (include all points).

(between 0.4-1 $\mu \mathrm{m}$ ) but the received 3D image showed an elongation effect which was a general error of the CLSM setup which had to be corrected. Monodispersed glass beads and slightly adhered GUVs were used to calculate a correction factor $F_{\text {corr }}=L_{x y} / L_{z}$ for the $z$-stacks with the measured length $L_{x y}$ in $x y$-plane and $L_{z}$ in $z$-plane to receive round glass beads as can be seen from a scanning electron microscopy image in figure $3.21 \mathrm{~A}$. 
A measurement with the above mentioned CLSM of monodispersed glass spheres with a size between $3.2 \mu \mathrm{m}$ and $7.3 \mu \mathrm{m}$ revealed that for small image stacks an average correction factor $F_{\text {corr }}=0.74 \pm 0.02$ ( \pm standard deviation) for the 3D image voxel depth is needed for the correction which is shown in figure 3.21 .

In figure 3.22 A the original data of a 3D image of a GUV is shown. The adhered GUV is strongly elongated and does not depict the real geometry. The average correction factor of $F_{\text {corr }}=0.80 \pm 0.02$ for large objects like GUVs has been found during the measurements of 3D images (Figure 3.21). With this factor all image stacks of GUVs were corrected which is exemplary depicted in figure $3.22 \mathrm{~B}$.
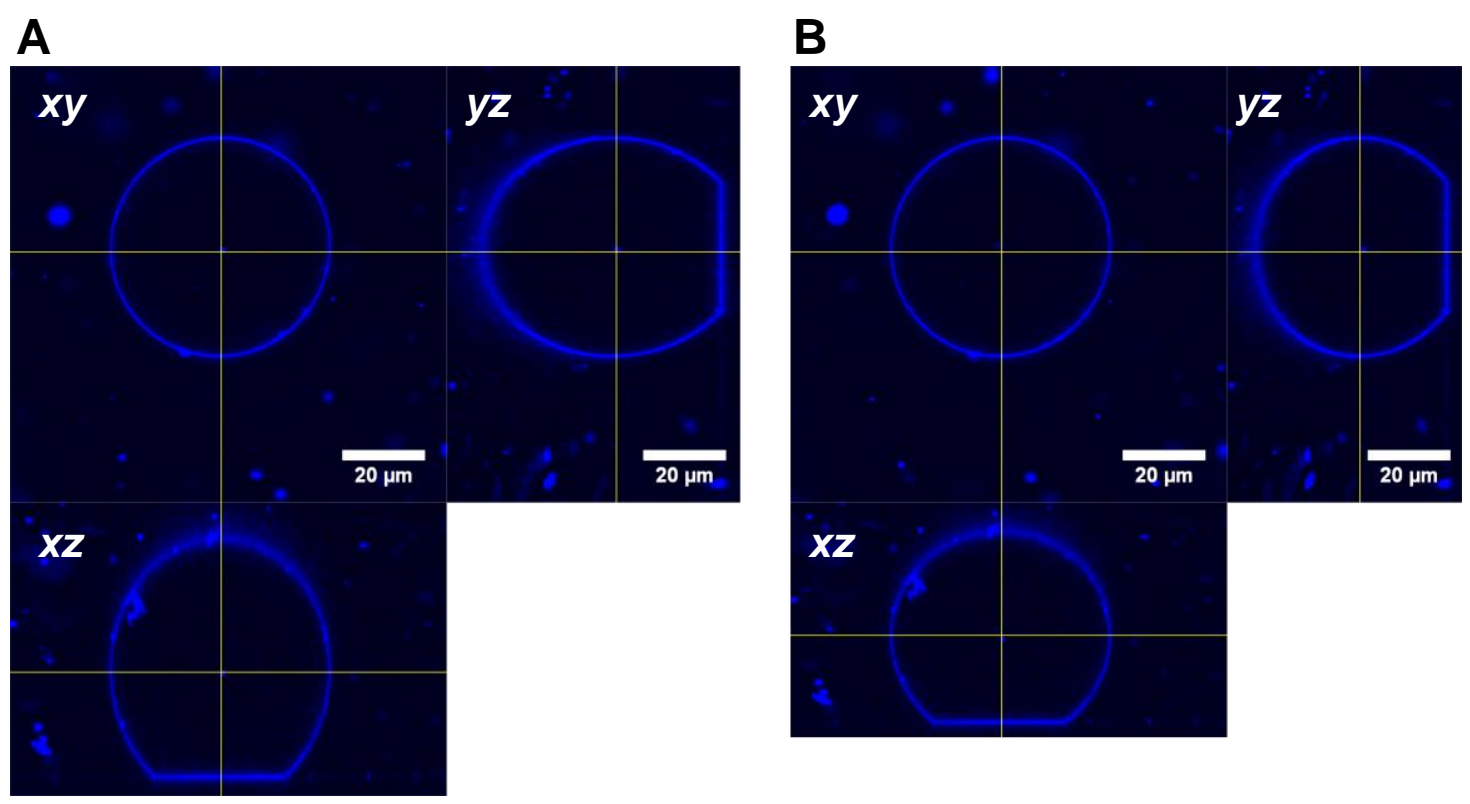

Figure 3.22. Correction of distances between focal planes for 3D images of GUVs. A) Original z-stack cross-sectional views $(x z, y z)$. The GUV is elongated along the $z$-axis. B) Correction of the image stack with factor 0.8 for voxel depth led to the reduction of height and a more realistic representation of the GUV geometry. 


\subsubsection{Fluorescence Recovery after Photobleaching}

Fluorescence Recovery after Photobleaching (FRAP) is a well-established method for the measurement of two dimensional diffusion coefficients of small molecules in thin and fluid films or lipids in membranes. ${ }^{105}$ Diffusion between two membrane coated micrometer glass spheres can be measured when connected by a fusion stalk between the two membranes. ${ }^{29}$ FRAP measurements therefore allowed to determine the size of the contact zone between the membrane coated spheres. The geometrical restriction of minimizing the contact zone produced a slowdown in diffusion of lipids over the hemi-fusion stalk with a recovery time between 20-30 minutes. Bleaching of a fluorescently labeled lipid dye in a SLB results in a much faster recovery of fluorescence intensity at the bleached area because of the fast and unhindered diffusion of the fluorescently labeled lipid dye from the surrounding unbleached membrane area. Typical diffusion coefficient $D$ of fluid phase bilayers are in the range of 1 to $10 \mu \mathrm{m}^{2} \mathrm{~s}^{-1} \cdot{ }^{70}$

FRAP provides information about the diffusion coefficient and whether the dye molecules are mobile or immobile. This information was significant because membrane fusion had to be verified by measuring the LUV dye at the SLBs. Docking of immobile LUVs on the SLBs could be partially excluded when a fast recovery of the LUV dye on the SLBs occurred after bleaching.

Experimentally, a high energy LASER pulse was used to bleach a region of interest (ROI) on the supported lipid bilayer with the actual bleaching area radius $r^{2}$. After the bleaching a recovery can be detected if the fluorescently labeled lipids are mobile in the SLB. The diffusion of these lipid dye molecules from the unbleached membrane area can be measured as a fluorescent intensity curve which is shown in figure $3.19 \mathrm{E}$. Out of the recovery curve of the fluorescence intensity the half-life of recovery $t_{1 / 2}$ could be read. With the LASER bleaching area radius $r^{2}$ the diffusion coefficient $D$ could be calculated with the following equation (14) which was found by SOUMPASIS et al.:106

$$
D=0.224 \cdot \frac{r^{2}}{t_{1 / 2}}
$$

Equation (14) was used for the analysis of FRAP measurements in this thesis to determine diffusion coefficients of the SLBs on the PDMS substrates and adhesion areas of the GUVs on functionalized glass substrates.

In figure 3.19 an exemplary FRAP measurement is shown that depicts the bleaching and recovery of fluorescence intensity in the SLB on the PDMS surface. This FRAP-measurement was done by Jörn Dietz as a part for his master thesis. The LASER bleached a very large spot that recovers with lipid dye after a few seconds. However, the fluorescence intensity at the bleached spot did not recovered fully to its initial intensity value because of the immobile fraction of lipids in the membrane. 

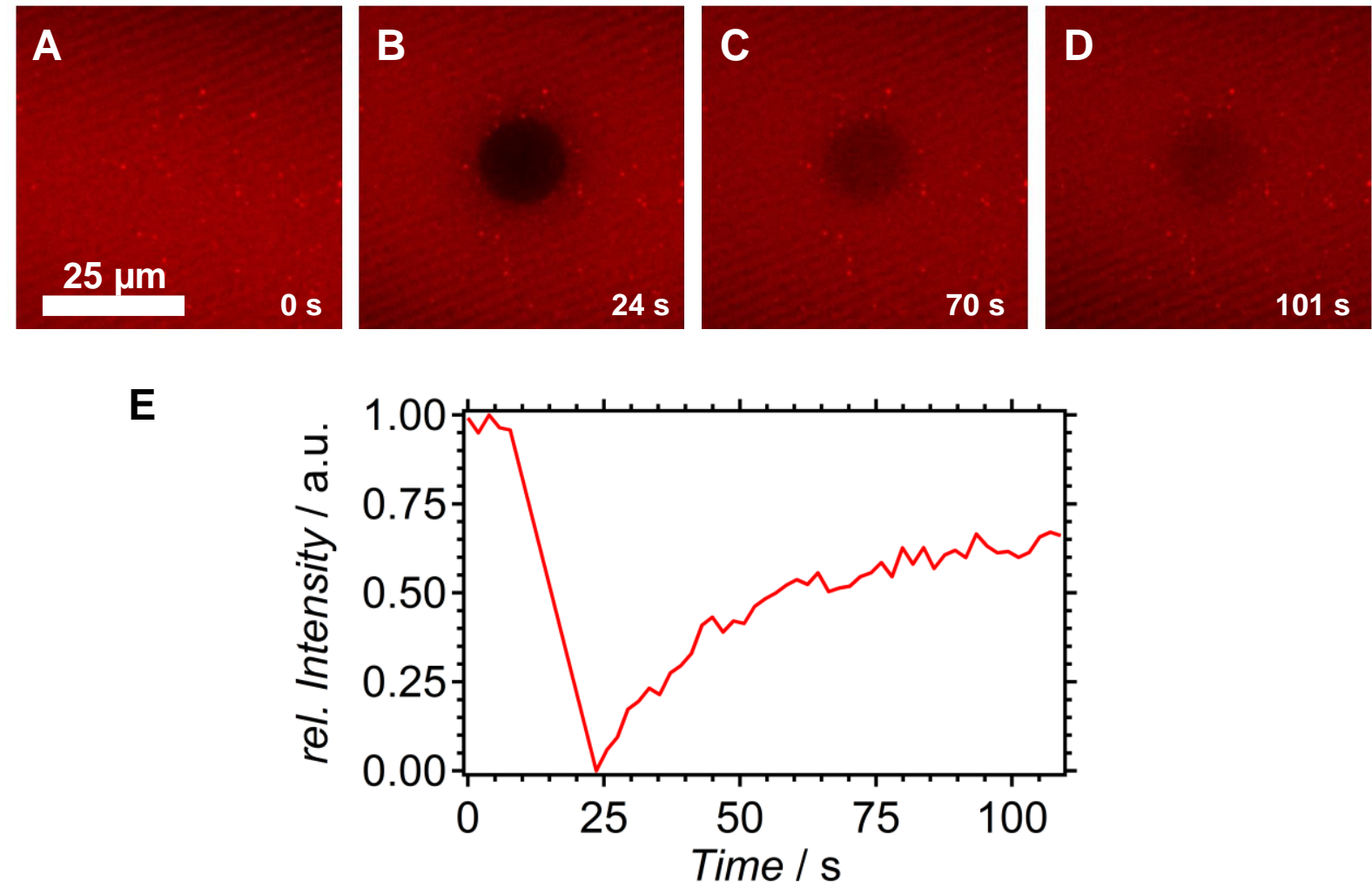

Figure 3.19. Exemplary FRAP measurements on a PDMS surface fully covered with a supported lipid bilayer containing the dye TexasRed ${ }^{\circledR}$ DHPE. A) The fluorescent images shown in a time series before and after bleaching. B) The $561 \mathrm{~nm}$ LASER bleached the dye lipid in the SLB in a large but defined region. C) Recovery occurs due to the diffusion of the fluorescently labeled lipids from the surrounding of the bleached area. D) After some time the recovery was finished. An immobile fraction reduces the fluorescence intensity compare to the surrounding and unbleached membrane. E) FPAP curve for the intensity of lipid dye at the bleached spot. 


\section{Experimental Section}

Two different experimental approaches were used to apply and increase membrane tension for the investigation of membrane fusion rates (Figure 4.1). Increasing membrane tension was achieved by a strong adhesion of GUVs to a substrate mediated by the avidin-biotin interaction and the divalent ion concentration $\left(\mathrm{Mg}^{2+} / \mathrm{Ca}^{2+}\right)$ in the buffer. In the second experimental approach, a polymeric substrate, PDMS, was used to create a device that can stretch a thin PDMS sheet between two side chambers by lowering the air pressure with a syringe pump. The adhered lipid bilayers on the PDMS sheet between the side chambers could then be dilated for 1 to $5 \%$ of their initial area which results in an increased membrane tension. In both experimental approaches, membrane fusion was investigate by incubating a LUV-solution for a defined time scale. Intensities of the two fluorescent dyes, originating from the two vesicle fractions, were compared to receive a fusion efficiency for a large variety of membrane tensions.

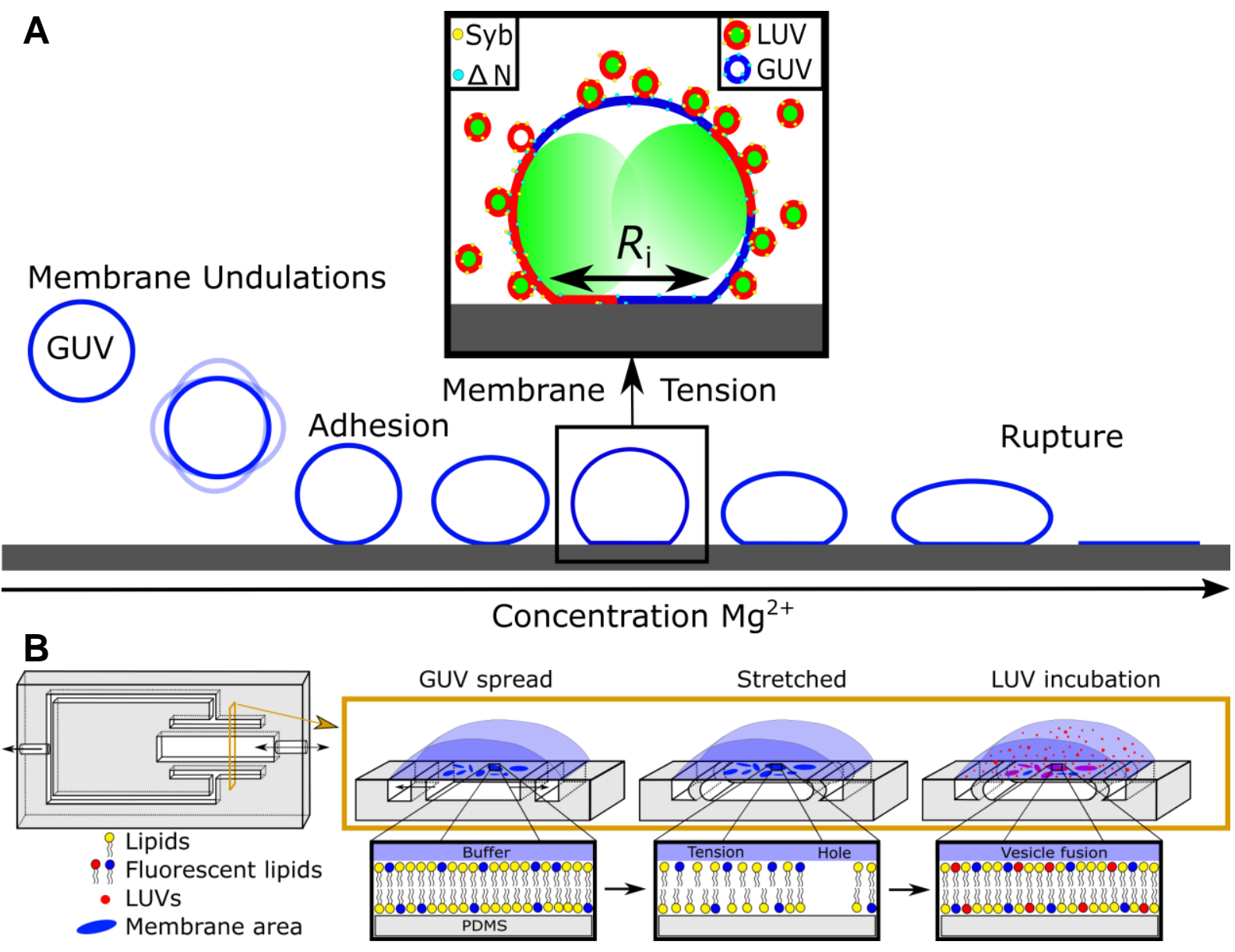

Figure 4.1. Two experimental approaches to investigate membrane fusion as a function of lateral membrane tension. A) Membrane tension is increased at GUVs that adhere to a surface. Larger adhesion radius $R_{i}$ generates larger membrane tension in the GUV membrane. LUV fusion experiments were done with adhered GUVs. B) The thin PDMS sheet on the fabricated membrane stretcher device was used as substrate for SLBs to dilate defined membrane patches and to investigate LUV fusion on tensed membranes. 
In figure 4.1 the two experimental approaches to measure membrane tension are illustrated. At first, giant unilamellar vesicles containing preassembled syntaxin 1 and SNAP 25 ( $\Delta \mathrm{N}$-complex) were incubated on a functionalize surface (Figure 4.1 A). The conditions between the inside of the GUV and the environmental buffer the same osmolality to prevent osmotic pressure generated by a difference in concentrations of dissolved molecules and salts. Due to increased divalent ion concentration, by adding magnesium chloride $\left(\mathrm{MgCl}_{2}\right)$, the adhesion area of the GUVs on the avidin coated glass surface was increased. Additionally, it should be mentioned that the buffer conditions stayed constant and the added buffer with the increased $\mathrm{MgCl}_{2}$ concentration had the same osmolality as the sample buffer. A larger adhesion area with the radius $R_{\mathrm{i}}$ generates a larger lateral membrane tension of the GUV membrane which can be calculated of the GUV radius $R_{v}$ and the adhesion radius $R_{i}$. (see chapter 2 ). Adhesion of GUVs on the functionalized glass surfaces suppressed membrane undulations that occur in freely floating GUV membranes so that the CLSM had been used to measure stacks of fluorescence images of adhered GUVs with different adhesion radii $R_{\mathrm{i}}$ without any interferences by membrane flickering. From the image stacks a three dimensional view of the GUVs can be achieved to visualize the geometry and afterwards the LUV fusion and docking efficiency in a two channel image. FRAP experiments on the adhesion area of GUVs served as a proof of membrane fusion and lipid diffusion between GUVs and LUVs.

The second experimental setup, shown in figure 4.1 B, consists of a milli-fluidic device which has a thin PDMS layer with a thickness of $180 \mu \mathrm{m}$ on top of the molded channels. Here, we generated global tension in SLBs by lowering the pressure in two adjacent side channels to dilate the PDMS surface in the middle which in turn can be used to increase the area of adhered lipid bilayers. The vesicle fusion efficiency as a function of the applied tension in the SLBs was monitored directly by fluorescence microscopy. The supported lipid bilayers were equipped with the $\Delta$ N49-complex and LUVs with synaptobrevin-2 (1-116) to obtain reasonable fusion activity. With this experimental setup, we could monitor the area change of each membrane patch after stretching the substrate and document the fusion of LUVs added to the solution. For the first time the impact of membrane tension on vesicle fusion was investigated with this model system. Membrane fusion was determined by the relative fluorescence intensity for each membrane patch and FRAP measurements confirmed fusion by bleaching the lipid dye of the LUV on the membrane patch that shows nearly full recovery if bleached. 


\subsection{LUV fusion to adhered GUVs}

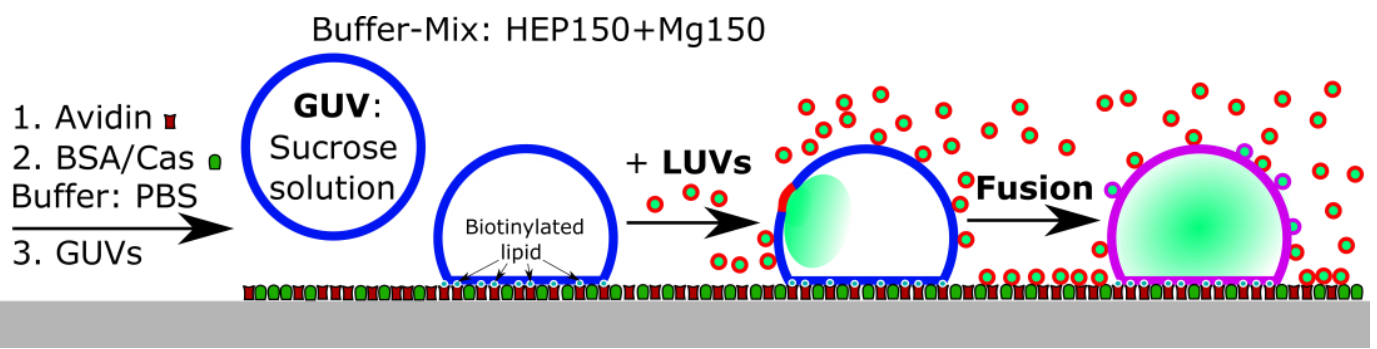

Glass

Figure 4.2. Scheme of GUV adhesion and LUV fusion experiment with SNARE proteins. The glass surface was covered with avidin and BSA or Casein. GUVs adhere via the avidin-biotin interaction to the surface. Membrane tension is generated by the adhesion strength of the GUV to the surface. Addition of LUVs results in membrane fusion between the GUV and LUV that is documented by lipid mixing and content mixing. This experiment provides the access to measure the fusion efficiency of LUVs to the GUV membrane as a function of membrane tension in the GUV membrane.

The first experimental approach to membrane fusion efficiency on membranes having a low or high membrane tension was to use GUVs that adhere to a surface with a defined adhesion strength that is responsible for the generated membrane tension in the GUV membrane. At first, the hydrophilic glass surface was functionalized with avidin ( $1 \mu \mathrm{M}$, in PBS) to create binding sites on the surface for the biotinylated lipids (figure 4.2). ${ }^{107}$ After the coating of a hydrophilic glass surface in a petri dish (5 mm Dish, 1.5 Coverslip, $14 \mathrm{~mm}$ Glass Diameter, MatTek Corporation, Ashland, MA, USA) with the phosphate-avidin buffer solution the phosphate buffer equipped with casein $(100 \mu \mathrm{M})$ (Cas) or bovine serum albumin (BSA) $(100 \mu \mathrm{M})$ was incubated on the surface to passivate the potentially uncovered space of the glass surface to inhibit uncontrolled spreading und rupture of the adhered GUVs. Because of the sucrose solution inside the GUVs, they settled down on the avidin-functionalized surface and started to adhere. A mixture of the buffers HEP150 and Mg150 was used to reach a defined $\mathrm{Mg}^{2+}$ concentration between $0.2-6 \mathrm{mM}$ in the sample with the GUVs. All experiments for the adhesion of GUVs on avidin-functionalized glass surfaces were carried out with buffer conditions (HEP150) that were adjusted to an osmolality of $(150 \pm 2)$ mOsmol kg-1 (see table 3.5 in chapter 3.2). A lower $\mathrm{Mg}^{2+}$ concentration resulted in a small adhesion area of GUVs and thereby in a lower membrane tension. If a higher $\mathrm{Mg}^{2+-}$-concentration had been adjusted then the GUVs adhered stronger to the substrate with a large adhesion area and consequently the membrane tension was increased. Evaporation of water from the buffer solution in the sample petri dish was prevented by surrounding the whole petri dish with a large glass petri dish and sealing it with a wet tissue to saturate the atmosphere with water vapor inside this chamber so that no osmotic changes of the buffer solution could create an osmotic pressure to the GUVs. For the addition of LUVs to the adhered GUVs in the petri dish a bent syringe needle was attached to the glass petri dish. Membrane tension therefore was only generated by the addition of divalent ions. At higher $\mathrm{Mg}^{2+}$-concentrations the adhesion strength was high enough to induce rupture of the strongly adhered GUVs that could spread to a flat supported lipid bilayer on the surface but also holes in the adhesion area occurred to reduce the membrane tension generated by a stronger adhesion. In figure 4.2 the whole experiment is drawn in an illustrated artwork to sum up the crucial events for the measurement of fluorescent images of adhered GUVs and their corresponding fusion efficiency with 
LUVs. As a reminder of the SNARE complex being used as a provider for the free energy to fuse membranes, with the new model system of adhered GUV the membrane tension was controlled by the concentration of divalent ions. Membrane fusion of LUVs to the adhered and tensed GUVs was mostly determined by lipid mixing. LUVs filled with $\mathrm{ATTO}^{\circledR} 488$ carboxy or pyranine, water soluble and very hydrophilic molecules, were used to determine the release of LUV content into the GUV where the fluorescence intensity of the used dye molecule increases if LUV fusion was present. 


\subsection{Extension of defined membrane areas on a PDMS substrate}

The general idea for the investigation of membrane fusion depending on lateral membrane tension of supported lipid bilayers is illustrated in figure 4.3 A. Therefore, the membrane stretcher was fabricated as described in chapter 3.5.2. A thin PDMS sheet on top of the molded PDMS layer containing the channel systems (figure $4.3 \mathrm{~B}$ ) served as a polymeric support that was dilated by a reduced air pressure in the side channels. Membrane patches on the PDMS were created by spreading of GUVs in a hyperosmotic buffer compared to the GUV content solution. Passivation of the residual PDMS surface was achieve by covering it with BSA. In order to change the available membrane area within a small range of $0-5 \%$ of its initial area we generated global tension in supported lipid bilayers by using a millifluidic device, which provides access to the full range from slightly negative to lysis tension with high accuracy.

Membrane tension was adjusted by applying a vacuum to adjacent side channels of this device that dilated the PDMS surface with the supported lipid bilayers. The membrane area change of membrane patches was measured using an optical microscope in conjunction with the thresholding technique of Ll et al. implemented in the open source software ImageJ.62, 108-109 In figure 4.3 B the area change of a membrane patch is shown with the corresponding lipid bilayer cross sectional view. If the membrane area increased then the lipids in both layers of the membrane gain more space and thus the whole membrane receives a higher membrane tension. Just by a measure of area change the membrane tension was calculated with equation (6) for each membrane patch. LUVs were incubated on stretched PDMS surfaces displaying membrane patches from ruptured GUVs until fluorescence images of relaxed and stretched membrane patches were taken.

To achieve membrane fusion LUVs were incubated on the stretched PDMS surfaces for around 30-45 minutes. Images of relaxed and stretched membrane patches were taken with the confocal microscope. LUV fusion on membrane patches with the determined membrane tensions was analyzed by a comparison of the two lipid dyes in the SLB (A390) and LUV (A594). As a measure for membrane fusion efficiency ( $F_{\text {eff) }}$ the fluorescence intensity of the lipid dye A594 (Luv, A594), originating from the LUVs, was compared to the intensity of the blue dye A390 (ISLB, A390) emitted from the planar membrane patches serving as an arbitrary reference $(100 \%)$.

$$
F_{\text {eff }}=\frac{I_{\mathrm{LUV}, \mathrm{A} 594}}{I_{\mathrm{SLB}, \mathrm{A} 390}} \cdot 100 \%
$$

The fusion efficiency of each SLB was set in reference to the membrane tension of each SLB that was calculated by equation (6) comprising the measured area change. Furthermore, LUVs were classified as docked if they had not shrunken by incorporation of the lipids into the SLBs and at the same time had not displayed fluorescence from the blue dye A390. Hemi-fused vesicles do not shrink but show fluorescence from A390. FRAP was carried out to confirm LUV fusion on SLBs. Therefore, the dye A594 added to the LUVs was bleached with full laser intensity for a few seconds and images were taken to record the recovery of the lipid dye into the bleached spot. This system allows for the first time to quantitatively investigate the impact of membrane tension on vesicle fusion. In the following subchapters the preparation of the PDMS membrane stretching device and all experiments with this device are described explicitly. 

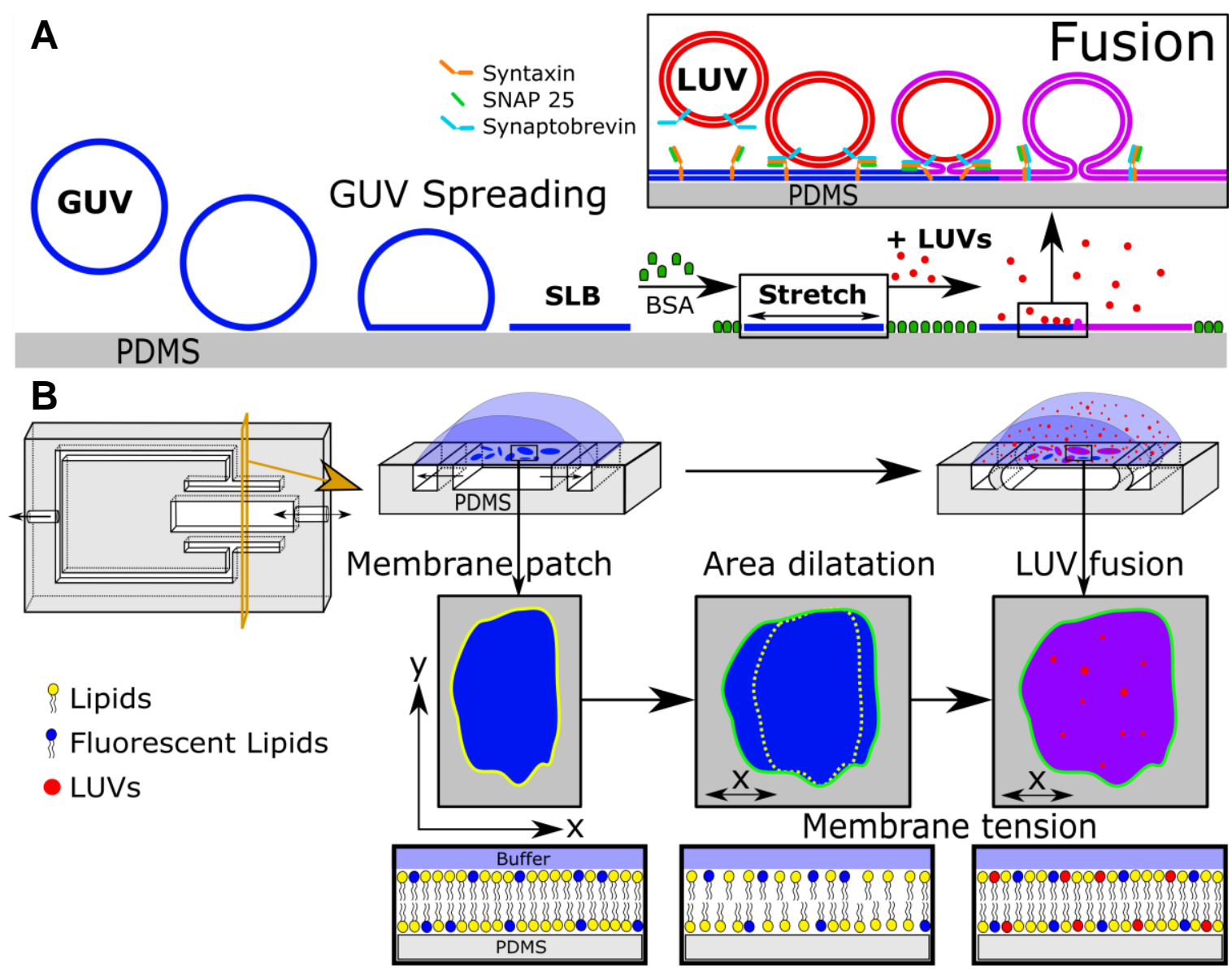

Figure 4.3. General experimental process to investigate LUV fusion to supported lipid bilayers (SLBs) depending on the area change induced by the dilatation of the PDMS support. A) GUVs were spread on a hydrophilic PDMS surface in HEPES buffer to form defined areas of membrane patches. Passivation of the uncovered PDMS surface was achieved by incubation of BSA in PBS solution inhibiting LUV fusion on the naked PDMS. Stretching of the SLBs was carried out by the dilatation of the PDMS support and membrane fusion of LUVs to the tensed SLBs was mediated by the presence of SNARE proteins. B) A schematic illustration of the membrane stretching device and its cross-sectional view is depicted. A large central chamber with a connection to the surrounding has two adjacent side channels. If the pressure in the side channels is reduced then the thin PDMS sheet in between dilates and supported lipid bilayer patches are stretched between $0-5 \%$ of their initial area that generates the membrane tension. Docking, hemi-fusion, and full-fusion of LUVs to the SLBs were monitored via fluorescence microscopy. 


\subsubsection{Preparation of membrane coated PDMS surfaces}

At first, the surface of the thin PDMS layer of the chamber was oxidized in an oxygen plasma for ten seconds with a Zepto plasma generator (Diener, Ebhausen, Germany). Only the inner part of the PDMS network that can be stretched was oxidized. Aluminum foil was placed around that area to protect the other surfaces from being oxidized. Secondly and directly after the plasma oxidation, $500 \mu \mathrm{L}$ of HEP220buffer (table 3.7 in chapter 3.2) was added onto the surface to maintain the hydrophilic character. The methyl-siloxanes on the surface were oxidized with oxygen plasma so that a thin glass layer is created on top of the PDMS network. In figure 4.4 the surface oxidation and preparation with supported lipid bilayers is visualized in a schematic drawing of the whole process of surface functionalization.

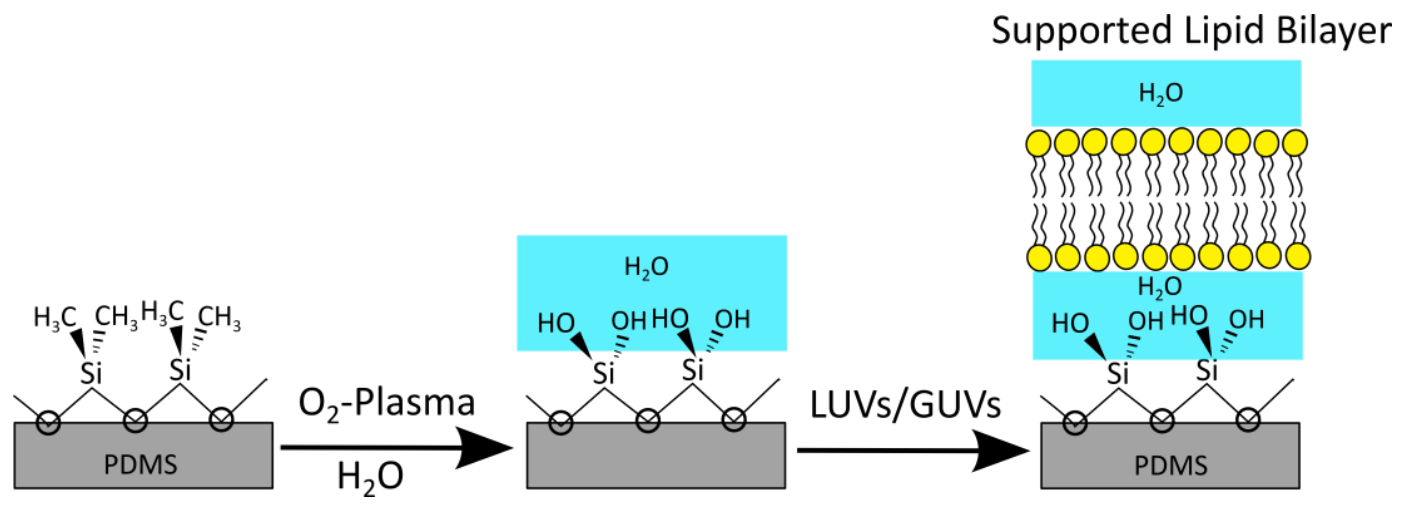

Figure 4.4. Production of hydrophilic PDMS surface. The surface of the thin PDMS sheet of the membrane stretcher device was oxidized for ten seconds in oxygen plasma. Incubation of LUVs leads to spreading of them on the PDMS surface producing a surface fully covered with a SLB. Incubation of GUVs leads to defined membrane patches.

Giant unilamellar vesicles were produce by the electroformation method in a sucrose solution (220 mM) and LUVs by the detergent dilution method both described in chapter 3.4. LUV spreading on hydrophilic PDMS led to the formation lipid bilayer that covers the whole surface. In contrast, to facilitate the spreading of GUVs that adhere to the hydrophilic PDMS surface, a high ionic strength buffer HEP3 (table 3.8 in chapter 3.2) was introduced into the system to create a hyperosmotic environment that facilitates GUV membrane rupture to form isolated and defined membrane patches on the hydrophilic surface. A sample without passivation of the PDMS surface with BSA showed spreading of LUVs on the surface and membrane fusion between spread LUVs and SLBs by mixing of the two different lipid dyes. Therefore the passivation of the uncovered and hydrophilic PDMS surface was achieved by incubating the system for around 10-15 minutes in phosphate-buffer PBS (table 3.4 in chapter 3.2) equipped with BSA $(0.1 \mathrm{mM})$ to fill the uncovered and hydrophilic PDMS surface with BSA proteins that adhere to the surface (figure 4.3). Therefore, unspecific LUV fusion to the SLBs was inhibited by this passivation with BSA and activated in the presence of SNAREs. 


\subsubsection{Measurement of supported lipid bilayer dilatation}

Investigation of membrane tension on stretched SLBs was achieved by a dilatation of the polymeric support of SLBs. Such a support was build up by a fabricated membrane stretcher device (see chapter 3.5.2) that holds a thin, freestanding PDMS sheet on top of a PDMS layer containing the channel system. Two side channels next to the main chamber of the PDMS substrate (figure 4.3 in chapter 4.2) were connected via a flexible tube and luer lock connectors to a gas tight syringe $(10 \mathrm{~mL}$, Hamilton, Reno, NV, USA) that was controlled by a syringe pump (Fusion 200, KR Analytical, Sandbach Cheshire, UK). After preparation of supported lipid bilayers (SLBs) on the PDMS, the syringe pump generated a lower pressure in the connected tube and PDMS channel system by increasing the volume of the air by withdrawing the stamp of the syringe for 1-4 $\mathrm{mL}$. A lower pressure induced a small stretch of the PDMS surface between the side channels. To measure the strain that is exerted on the PDMS surface, small glass beads have been used to investigate how the PDMS surface area is influenced by the one directional strain. The surface dilatation $\epsilon_{\mathrm{x}}$ was defined as the distance change $I I_{0}$ between clusters of glass beads with the following equation

$$
\epsilon_{\mathrm{x}}=\frac{I-I_{0}}{I_{0}}
$$

where $/ 0$ stands for the initial distance and / for the distance after surface extension. A typical surface extension was measured between two clusters of beads (figure 4.5) after surface extension by reducing air pressure in the side channels through a connected syringe pump. As a result, the surface dilatation in percent was measured as a function of syringe volume increase which is shown in figure $4.5 \mathrm{~B}$. This finding was helpful to determine an applicable range of surface area extension of membranes that is below the lysis tension of $10 \mathrm{mN} \mathrm{m}^{-1}$ which refers to an area increase of about $3.6 \%$ of its initial area (see chapter 2 ). With this system a surface dilation between $0-5 \%$ can be achieved and measured very precisely. For this purpose a maximum syringe volume increase up to around $1.5 \mathrm{~mL}$ was defined. Note that the membrane patches on a PDMS surface comprise a small area and the surface dilatation is distributed anisotropically over the whole surface with a minimum in the middle of the extended surface
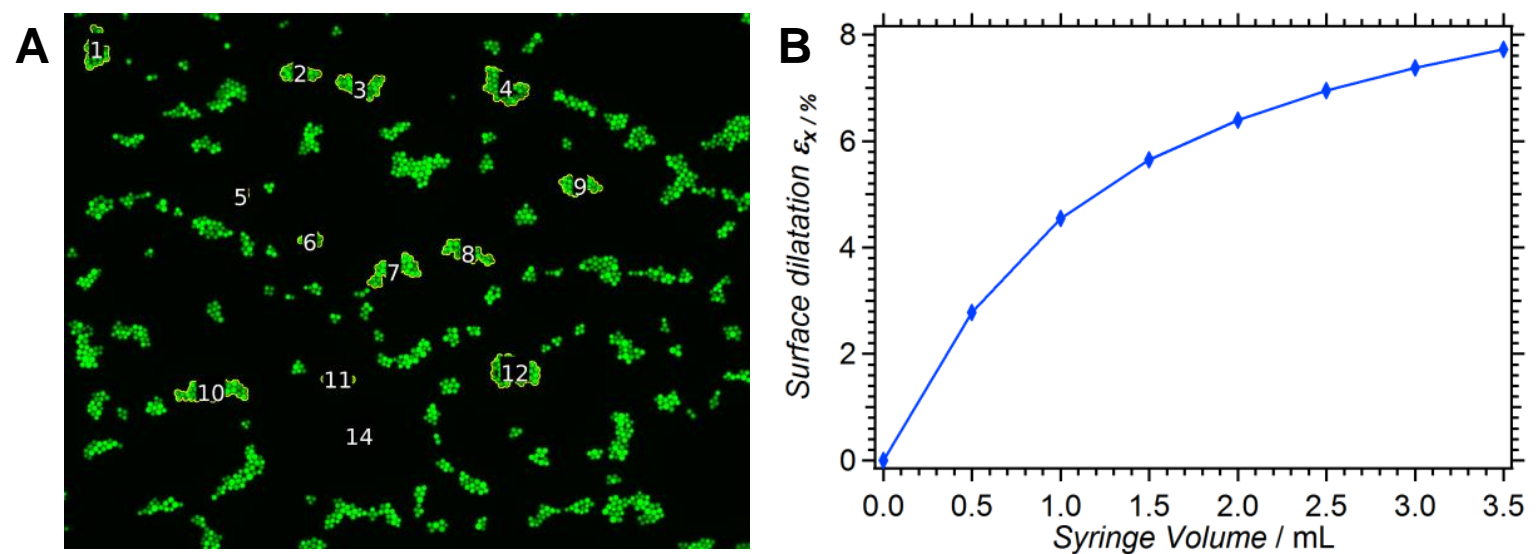

Figure 4.5. Dilatation of PDMS surfaces with supported lipid bilayers. A) Glass beads (green) were introduced unto the PDMS surface to measure the distances between the bead clusters. B) If the PDMS sheet was dilated by an increased syringe volume using a $10 \mathrm{~mL}$ gas tight syringe that is connected to the side channels then the distance between the glass bead clusters increases. Lines between the points serve as guideline for the eyes. 
sheet between the side channels. The extension of the surface increases from the middle to the side channels which was also demonstrated by Huh et al. for their PDMS cell stretcher with quantum dots on a patterned PDMS sheet. ${ }^{61}$ For the purpose of this thesis a surface extension was achieved by a syringe volume increase between $1.5 \mathrm{ml}$ and $2.0 \mathrm{~mL}$ to reduce air pressure in the side channels.

The question now was how to measured and define the region of interest $(\mathrm{ROI})$ where the relevant membrane area can be measured. In 1993 Li and Lee introduced the minimum cross entropy thresholding algorithm that minimizes the cross entropy between a segmented image and the original image and in 1998 a fast iterative method was established that is implemented in the Image J software. ${ }^{62}$, 108-109 The well-established LI-thresholding technique is the best method to detect the area of membrane patches automatically because of the high accuracy even if there was an intensity decline through the image series through bleaching of the lipid dyes by the scanning LASER. It was also no problem for the used thresholding technique to handle with a small uneven intensity distribution in one picture that barely occurred. But to reduce further impact of an uneven intensity distribution from the very large images $\left(\sim 1600 \times 1600 \mathrm{pix}^{2}\right)$ that were taken to measure many membrane patches simultaneously a small square around the membrane patch with enough background was cut out of the large image and the Lithreshold was applied for the membrane patch on the small cut image. This was done to reduce the noise and to detect the threshold region more precisely since the LI-thresholding technique uses the grey-level histogram for its algorithm and the grey-level intensity distribution is shaper in a smaller region cut off from the larger image.

For the analysis of the membrane area changes the above described minimum cross entropy thresholding technique implemented in the ImageJ software was used. Therefore, the membrane area dilation $\epsilon_{\mathrm{m}}$ for each membrane patch was calculated with the following equation:

$$
\epsilon_{\mathrm{m}}=\frac{A-A_{0}}{A_{0}}=\frac{\Delta A}{A_{0}},
$$

where $A_{0}$ denotes for the initial membrane area before and $A$ for the membrane area after the PDMS surface dilatation. With equation (17) the area increase of each membrane patch was calculated in percent by multiplying with $100 \%$.

In figure 4.6 A and B, a large membrane patch before $(A)$ and after the surface extension (B) is shown. For the displayed membrane patch in figure 4.6 an exceptional syringe volume increase of $3.0 \mathrm{ml}$ was applied to gain an area increase of $1.5 \%$ which is a result of the surface extension of the PDMS sheet. To visualize area change of the stretched membrane both ROls of the stretched and relaxed membrane areas are shown in both images. The axis of surface extension (x-axis) is given in figure 4.6 $\mathrm{A}$ and it occurs that the membrane patch after surface dilatation was stretched along the $x$-axis which documented the membrane area increase in particular. Over the whole experiment of stretching and incubation of LUVs images of the SLB were taken and analysed later on to measure the occupied SLB area on the PDMS which is shown in figure $4.6 \mathrm{C}$ as an example for the membrane patch in the same figure. Each data point in the graph represents the area measurement in one image so that a few images led to the boxplot and calculation of an average SLB area and its error. As a result, the average SLB area increased by $(1.4 \pm 0.1) \%$ due to the stretching of the PDMS-surface. 
This analysis technique was used for all fluorescent images to determine the membrane area change of the defined SLBs on the PDMS substrate surface for the subsequent calculation of membrane tension.
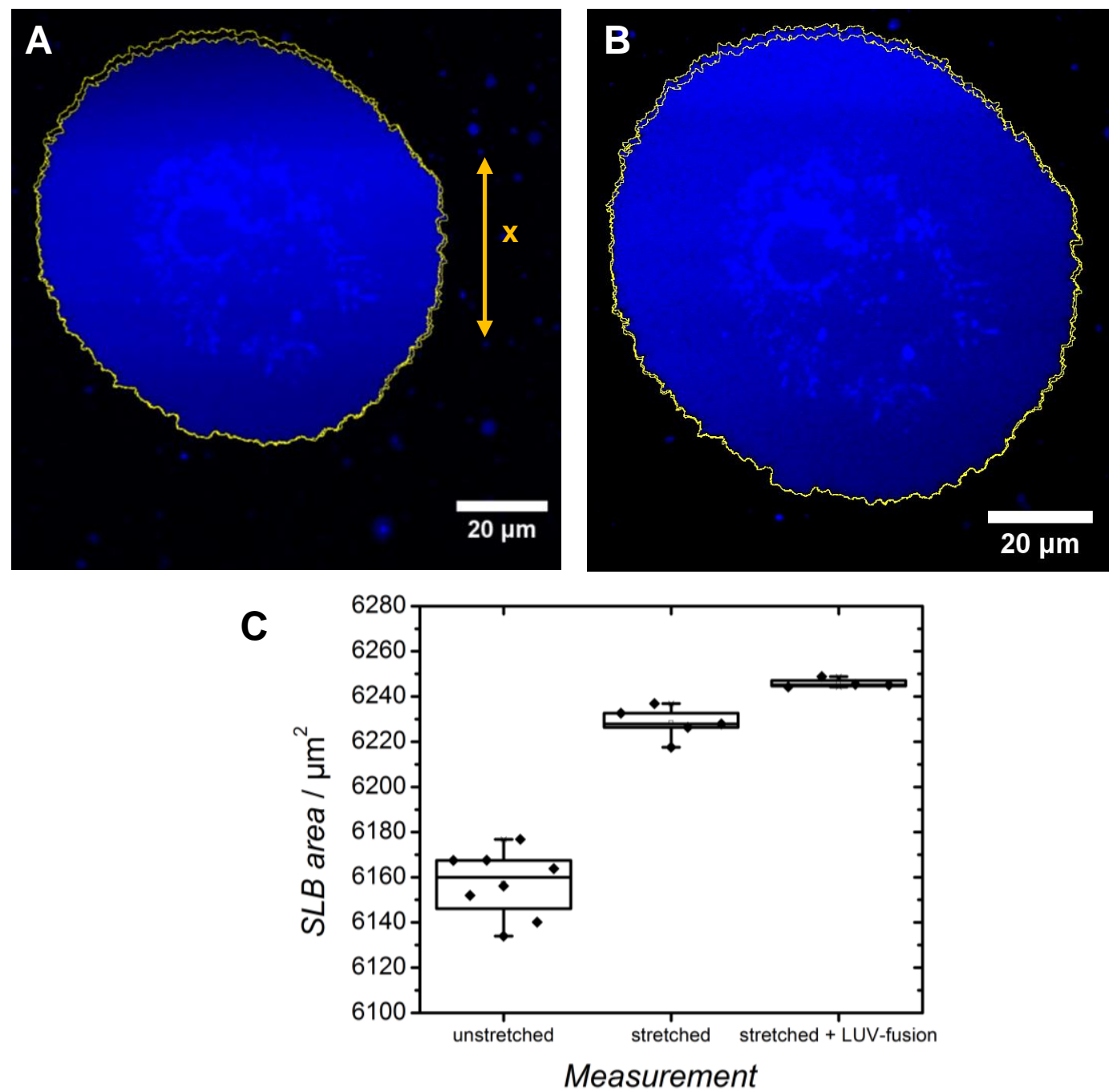

Figure 4.6 A) A large membrane patch with an average area of $6157 \mu \mathrm{m}^{2}$ before surface dilatation. Two ROls (yellow) have been drawn into the picture that show the actual and increased membrane area before and after surface area increase. B) The scanned image with a stretched membrane patch with and the ROls before and after stretching shows an area increase of $1.5 \%$ after stretching of the PDMS surface to an average area of $6229 \mu \mathrm{m}^{2}$. C) The boxplot shows the measured data points and the average SLB area. The SLB area increases due to the PDMS stretching and slightly through LUV fusion. 


\section{Results}

\subsection{LUV fusion to adhered and tensed GUVs ${ }^{4}$}

A common vesicle fusion assay was chosen to investigate the fusion of LUVs to adhered GUVs on a functionalized glass surface. As described in the chapter 4.1., the GUVs were incubated on an avidinfunctionalized glass surface so that the lipid cap-biotinyl-DOPE in the GUV-membrane can interact with the avidin on the glass surface by forming an avidin-biotin complex. Thus a non-covalent but strong binding of the GUV-membrane to the surface is obtained. By applying a low concentration of $\mathrm{Mg}^{2+-i o n s}$ the adhesion area was rather small and thus the induced membrane tension was also small. A large adhesion area was a result of an increased concentration of $\mathrm{Mg}^{2+-i o n s}$ in the buffer that mediates the interaction of the GUV-membrane to the avidin-functionalized glass surface. A 3D-image of the adhered GUVs was achieved by measuring image stacks with the confocal laser scanning microscope so that the geometry of the GUVs can be extracted from that images and cross-sectional views. LUVs were added to the sample to measure the fusion dependency by a measure of fluorescence intensity increase of the fluorescently labeled lipids originating from the LUVs at the GUV-membrane.

In this chapter two approaches are described providing membrane fusion between the adhered GUV and the incubated LUVs in the system. At first, vesicle fusion between LUVs and adhered GUVs with adjusted membrane tension was investigated. Via fusion the fluorescently labeled lipids from the LUVs diffuse into the GUV-membrane. In the second experiment LUVs, containing a green fluorescent dye (ATTO ${ }^{\circledR} 488$ ), were added to a sample with adhered GUVs to prove full fusion through a fluorescence intensity increase in the GUV. This experiment content mixing of LUVs reveals that the lipid bilayer of both vesicles can fully fuse.

\footnotetext{
${ }^{4}$ Partly published in:

Gleisner, M.; Kroppen, B.; Fricke, C.; Teske, N.; Kliesch, T.-T.; Janshoff, A.; Meinecke, M.; Steinem, C., Epsin N-terminal Homology Domain (ENTH) Activity as a Function of Membrane Tension. Journal of Biological Chemistry 2016, 291 (38), $19953-$
} 19961. 


\subsubsection{Lipid mixing of LUVs with adhered GUVs}

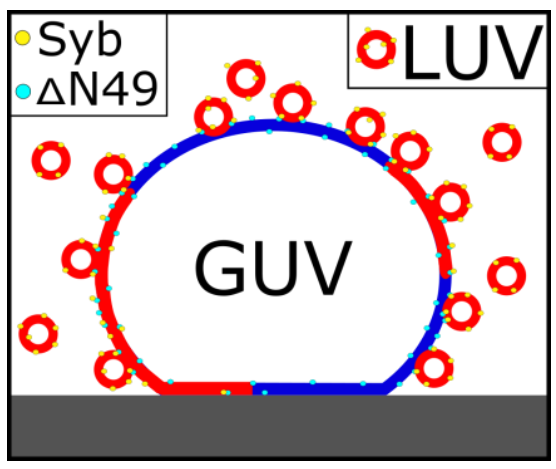

Figure 5.1. Mixing of lipids between the GUV and LUVs was achieved by membrane fusion induced by SNAREs and membrane tension so that the fluorescent dye of the LUVs diffused into the membrane of the GUV.

Lipid mixing between the membranes of the LUVs and the adhered GUVs on the functionalized surface, illustrated in figure 5.1, is induced as a fact of the adjusted membrane tension. GUVs were composed of DOPC, DOPE, DOPS, cholesterol, cap-biotinyl-DOPE and a fluorescently labeled lipid, A390 or TexasRed ${ }^{\circledR}$ DHPE (see chapter 3.1). LUVs contained the same lipid composition, except cap-biotinylDOPE was left out. Two different fluorescently labeled lipid dyes were added to the membranes of the GUVs and LUVs. LUVs contained the fluorescently labeled lipids A594 or A488 and the GUVs the fluorescently labeled dye A390 or TexasRed ${ }^{\circledR}$ DHPE. The fluorescence intensity increase of the LUV dye at the GUV-membrane served as measure for the docking and fusion of LUVs. For a better visualization the brightness of the images in the figures of this chapter was increased. The incubation time of LUVs in the sample was set to be in between two and 20 minutes because the LUV docking and fusion occurred very fast due to the high collision rate of the LUVs with the GUVs at room temperature. Membrane fusion between the large and giant vesicles was mediated by the SNARE proteins that were incorporated into the membranes as described in chapter 3.4. The LUVs contained synaptobrevin, and the GUVs the $\Delta$ N49-complex.

By the increase of adhesion area the membrane tension of the GUVs was adjusted as described in chapter 4.1. The described experiments in this chapter demonstrate that membrane fusion between large and giant vesicles depends on the lateral tension of the GUV-membranes. 


\subsubsection{Experiment 1: Low membrane tension}

In experiment 1 the GUVs were slightly adhered to the functionalized glass surface. The adjusted low membrane tension on the GUVs serves as a reference to highly tensed membranes to compare the docking and fusion efficiency of LUVs. Typical membrane tension in biological cells are in the range of $0.01 \mathrm{mN} / \mathrm{m}$ to $0.3 \mathrm{mN} / \mathrm{m} .{ }^{44}$ Therefore, the membrane tension of a total of $13 \mathrm{GUVs}$ was adjusted to a similar range between $0.17 \mathrm{mN} / \mathrm{m}$ and $1.2 \mathrm{mN} / \mathrm{m}$ this experiment $\mathrm{m}$. In the figures 5.2 to 5.5 all measured GUVs are shown in cross-sectional images. For the comparison of LUV docking and fusion on each GUV, the images on the left is a two channel image for both dyes to indicate whether docking of LUVs at the freestanding GUV-membrane occurred. On the right of the figures 5.2 to 5.5 the crosssectional image the channel for the LUV dye is shown to further analyze the docking or fusion of the LUVs to the GUV-membrane. Adhesion of LUVs on the sample surface occurred in all measurements and the fluorescence intensity of the adhered LUVs served as a reference for the docked LUVs on the GUVs. 

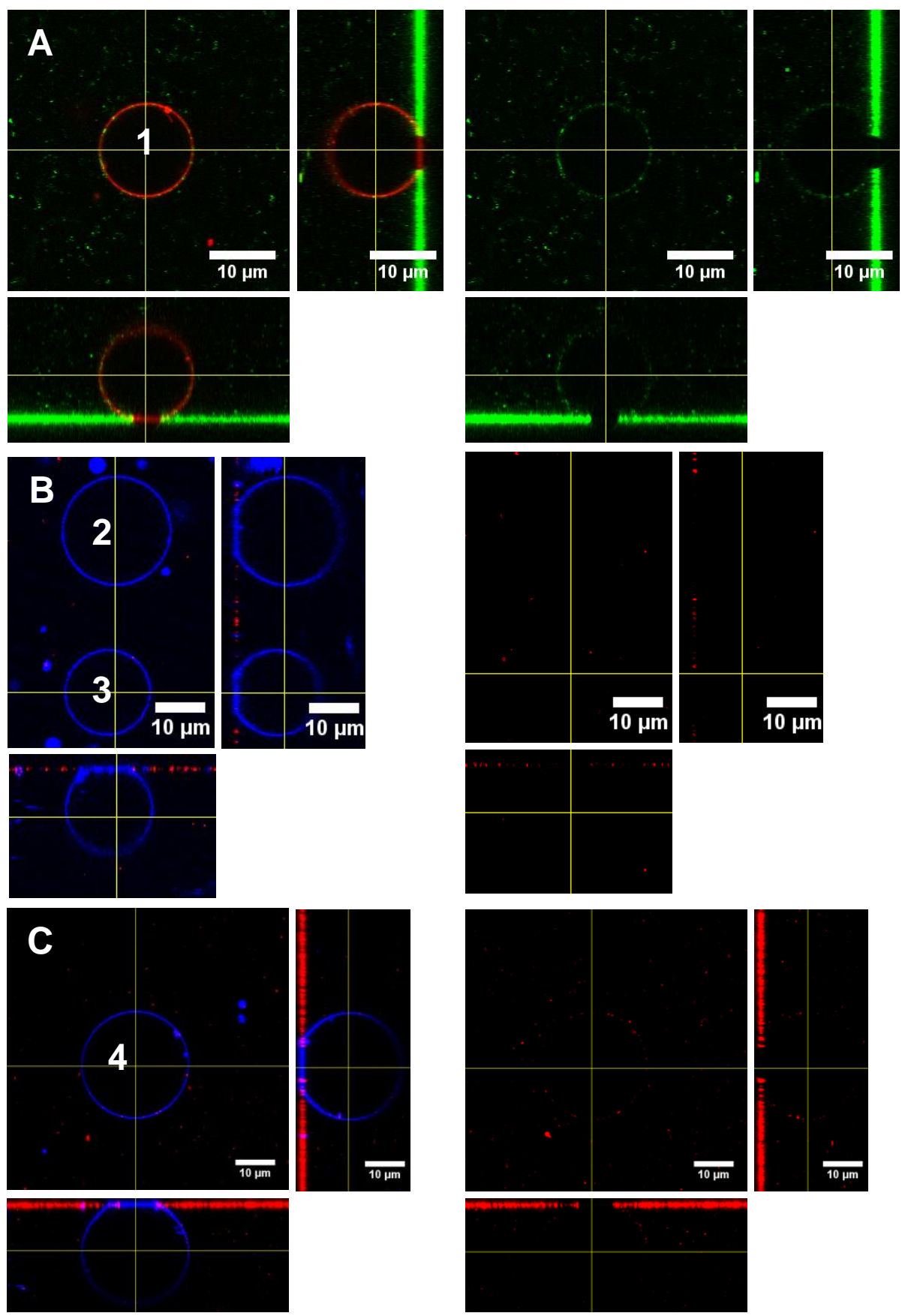

D

\begin{tabular}{|c|c|c|c|c|}
\hline GUV No. & $R_{\mathrm{i}} / \mu \mathrm{m}$ & $\widetilde{R}_{\mathrm{v}} / \mu \mathrm{m}$ & $\Delta A / A_{0} / \%$ & $\tau / \mathrm{mN} \mathrm{m}^{-1}$ \\
\hline 1 & 3.2 & 7.0 & 0.3 & 0.3 \\
\hline 2 & 4.6 & 10.8 & 0.21 & 0.23 \\
\hline 3 & 4.3 & 8.8 & 0.41 & 0.44 \\
\hline 4 & 7.1 & 13.5 & 0.5 & 0.56 \\
\hline
\end{tabular}

Figure 5.2. GUVs with low membrane tension and a few docked LUVs. A)-C) Left: Two channel images depicts GUVs and LUVs. Right: Only one channel for the LUV dye. Fluorescently labeled lipids in the GUV/LUV-membrane: A) TR (red) / A488 (green), B)-C) A390 (blue) / A594 (red). D) Table of measured radii, area changes and corresponding membrane tensions. 
In figure 5.2 , four slightly adhered GUVs with a membrane tensions between $0.3 \mathrm{mN} / \mathrm{m}$ to $0.56 \mathrm{mN} / \mathrm{m}$. To GUV 1, in figure 5.2 A, a few LUVs docked to the freestanding membrane but the adhesion site of the GUVs reveals no fluorescence intensity originating from the LUV dye. Compared to the fluorescence intensity of the LUVs on the sample surface the adhesion site of the GUV-membrane stayed dark. Only a few LUVs docked to the GUVs one to four shown in figure 5.2.

The GUVs 5-7 are shown in figure 5.3. Docking of LUVs to the GUV-membrane occurred mainly on GUV 5 with a larger adhesion radius compared to the other GUVs. Thus, the calculated membrane tension of $1.2 \mathrm{mN} / \mathrm{m}$ for GUV 5 is significantly higher compared to the other GUVs in figure 5.3. Interestingly, the adhesion site of GUV 5 shows no fluorescence intensity from the LUV dye. Therefore, LUV fusion at the membrane of GUV 5 did not occur. In figure 5.3 A and B, the surrounding solution of the adhered GUVs contained a higher concentration of LUVs compared to image $C$. The concentration of LUVs in each sample differed because the LUVs addition to the sample occurred locally and the LUVs could sink down to the sample surface so that the concentration of the LUVs in the solution was reduced through adhesion but even a high LUV concentration around the GUV in figure 5.3 B does not lead to an increased the docking and fusion to the membrane with a low membrane tension of $0.2 \mathrm{mN} / \mathrm{m}$.

A few more vesicles (GUVs 8-12) are shown in figure 5.4. The GUVs 8-11 in figure 5.4 were incubated with LUVs for 50 minutes to see whether LUVs dock to the membranes over time more frequently. Over such a long time an increased docking and fusion of the LUVs to GUVs with membrane tensions lower than $0.51 \mathrm{mN} / \mathrm{m}$ could not be detected.

In figure 5.5, GUV 13 with a membrane tension of $0.83 \mathrm{mN} / \mathrm{m}$ before the LUV addition is shown with many docked LUVs at the freestanding membrane. Compared to GUV 7 in figure $5.3 \mathrm{C}$ with a membrane tension of $0.78 \mathrm{mN} / \mathrm{m}$ GUV 13 the docking is significantly increased which could be related to a higher LUV concentration in the surrounding solution. Even a small amount of fusion to the GUV 13 is detected because of the slightly increased fluorescence intensity of the LUV dye A594 at the adhesion site of the GUV. The lipid mixing between the vesicles after fusion leads to the diffusion of the fluorescently labeled lipids into the adhesion area of the GUV. After the addition of the LUVs GUV 13 exhibited a larger adhesion radius which could have been induced by the friction forces while adding LUVs to the sample. However, between the values of $0.83 \mathrm{mN} / \mathrm{m}$ and $2.6 \mathrm{mN} / \mathrm{m}$ of membrane tension the docking and fusion of LUVs to the GUV-membranes is considerably elevated.

In summary to the results of experiment 1 , the docking probability of LUVs appears to be increased at membrane tensions above $0.8 \mathrm{mN} / \mathrm{m}$ as figure $5.3 \mathrm{~A}$ and 5.5 indicate. Membrane tensions below $1.2 \mathrm{mN} / \mathrm{m}$ on adhered GUVs do not lead to a measureable amount of fusion. 

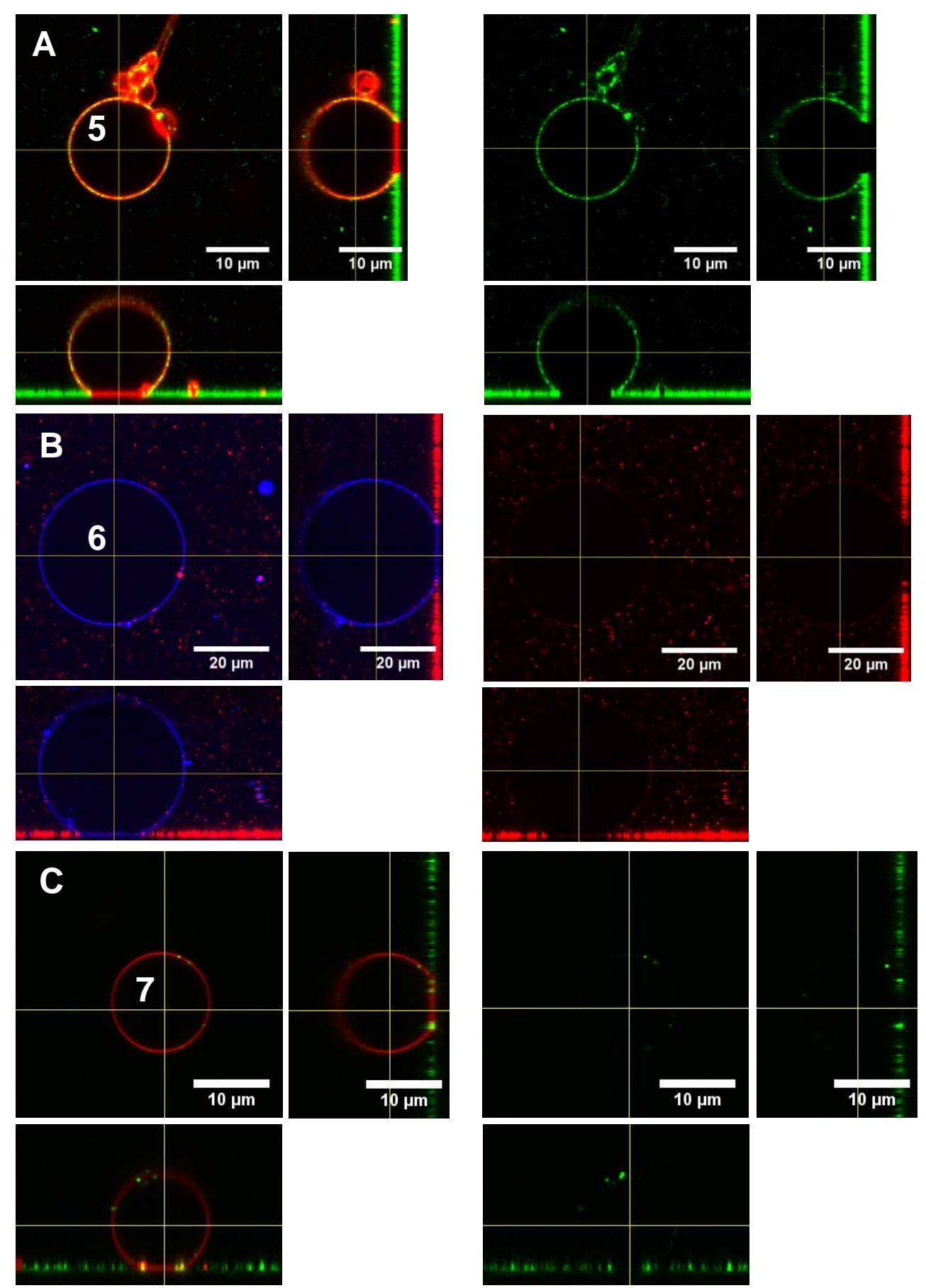

$\mathbf{D}$\begin{tabular}{|c|c|c|c|c|}
\hline GUV No. & $\boldsymbol{R}_{\mathrm{i}} / \boldsymbol{\mu m}$ & $\widetilde{\boldsymbol{R}}_{\mathrm{v}} / \boldsymbol{\mu m}$ & $\boldsymbol{\Delta} \boldsymbol{A} / \boldsymbol{A}_{\mathbf{0}} / \%$ & $\boldsymbol{\tau} / \mathbf{~ m N ~} \mathbf{~ m}^{-1}$ \\
\hline 5 & 4.7 & 7.9 & 0.85 & 1.2 \\
\hline 6 & 7.9 & 18.7 & 0.18 & 0.2 \\
\hline 7 & 3.6 & 6.5 & 0.64 & 0.78 \\
\hline
\end{tabular}

Figure 5.3. A)-C) GUVs with a low membrane tension and a few docked LUVs. Fluorescently labeled lipids in the GUV/LUV-membrane: TR (red) / A488 (green), A390 (blue) / A594 (red). D) Table of measured radii, area changes and corresponding membrane tensions. 


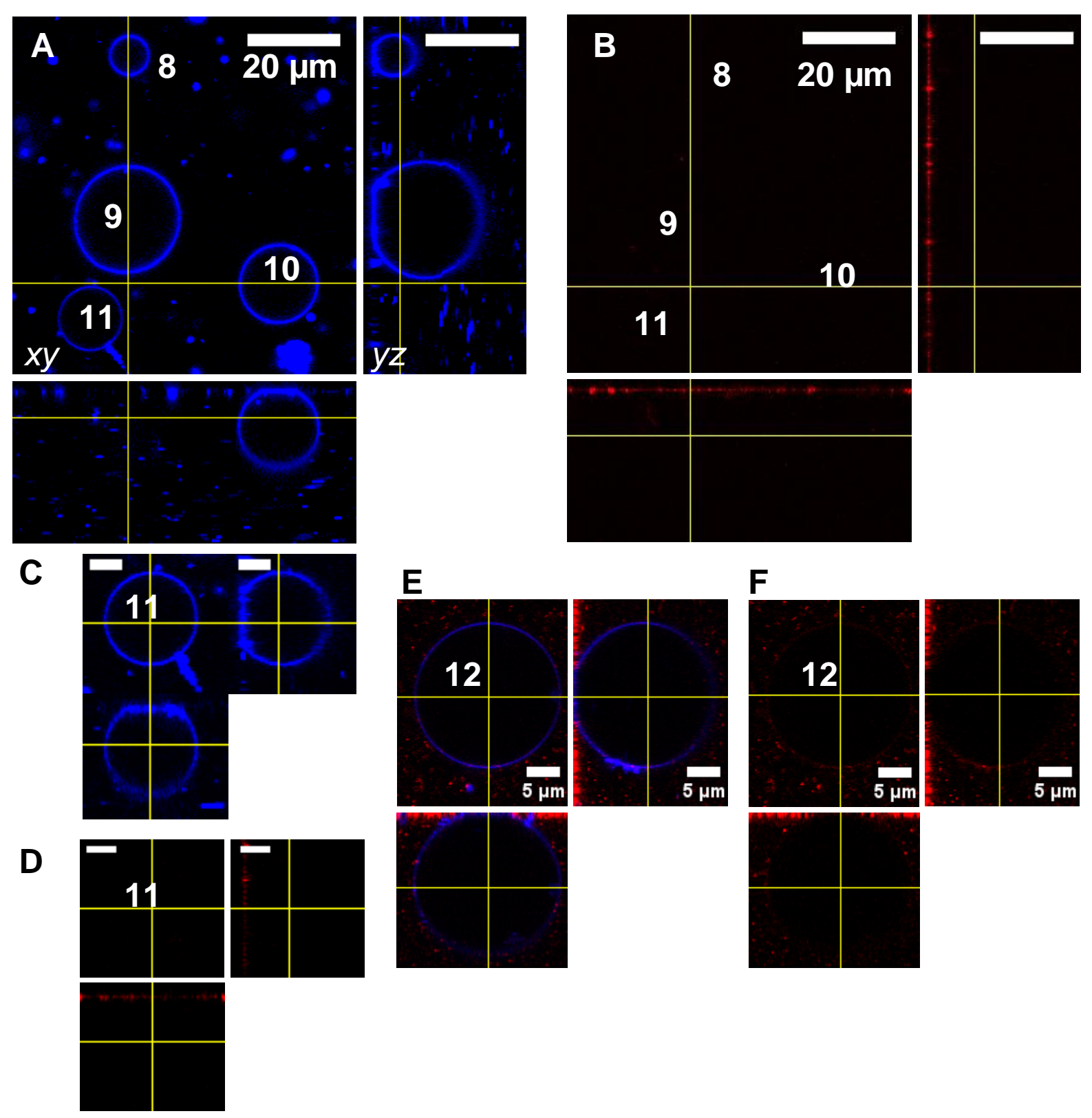

Figure 5.4. Slightly adhered GUVs with low membrane tension exhibit no LUV fusion. A), C), E) Crosssectional view of the GUV-membrane (blue) containing A390. B), D), F) The red channel of the LUV dye A594 shows no fluorescence on the GUVs but on the substrate surface. A few LUVs dock to the membrane or GUV 12. G) The table shows the measured radii and area changes of the GUVs and lists the calculated membrane tensions.

G \begin{tabular}{|c|c|c|c|c|}
\hline GUV No. & $R_{\mathrm{i}} / \mu \mathrm{m}$ & $\widetilde{R}_{\mathrm{v}} / \mu \mathrm{m}$ & $\Delta A / A_{0} / \%$ & $\tau / \mathrm{mN} \mathrm{m}^{-1}$ \\
\hline \hline 8 & 2.2 & 4.3 & 0.46 & 0.51 \\
\hline 9 & 5.6 & 12.5 & 0.26 & 0.27 \\
\hline 10 & 4.2 & 8.7 & 0.34 & 0.36 \\
\hline 11 & 2.6 & 6.4 & 0.19 & 0.21 \\
\hline 12 & 4.0 & 10.7 & 0.13 & 0.17 \\
\hline
\end{tabular}



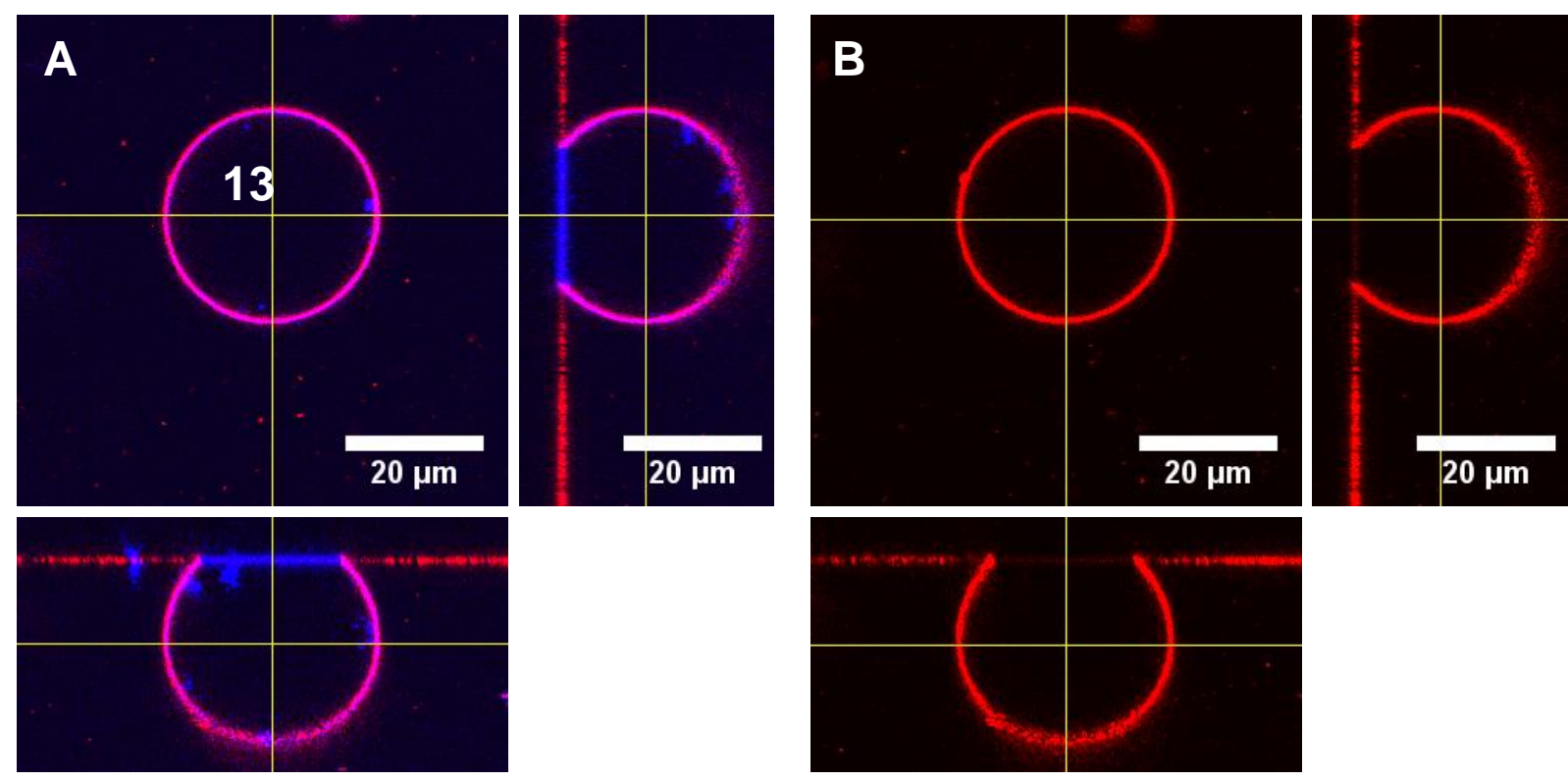

C

\begin{tabular}{|c|c|c|c|c|}
\hline LUV-addition & $R_{\mathrm{i}} / \mu \mathrm{m}$ & $\widetilde{R}_{\mathrm{v}} / \mu \mathrm{m}$ & $\Delta A / A_{0} / \%$ & $\tau / \mathrm{mN} \mathrm{m}^{-1}$ \\
\hline before & 8.5 & 15.1 & 0.67 & 0.83 \\
\hline after & 10.3 & 15.1 & 1.5 & 2.6 \\
\hline
\end{tabular}

Figure 5.5. A slightly tensed GUV. A) Both dyes for the GUV (blue) and the LUVs (red) are shown in an overlay image and the cross-sectional images on the right and bottom. B) The red channel depicts a high docking rate at the freestanding GUV-membrane and a slightly lower red fluorescence intensity at the adhesion area of the GUV. C) The table shows the measured radii and area changes of the GUV 13 for the calculation of membrane tension. 


\subsubsection{Experiment 2: Elevated membrane tension}

Comparable membrane tensions to those occurring in cells do not lead to a significant increase of vesicle fusion as it is described in experiment 1 . With the used vesicle fusion assay the tension of adhered GUVs was increased by adding divalent ions into the buffer of the sample. The interaction of the GUVmembrane with functionalized glass surface increased the adhesion area until the membrane reaches the lysis tension at which the vesicle ruptured. For experiment 2 eight GUVs with membrane tensions from $1.1 \mathrm{mN} / \mathrm{m}$ to $8.6 \mathrm{mN} / \mathrm{m}$ served as target membranes for the investigation of the docking and fusion efficiency of LUVs.

In the figures 5.6 to 5.9 the GUVs 14-21 with an elevated membrane tension are shown after the incubation of LUVs. For GUV 14 in figure $5.6 \mathrm{~A}$ the largest membrane tension of $8.6 \mathrm{mN} / \mathrm{m}$ for all measured GUVs was be calculated which is close to the lysis tension. The two channel image on the left side in the figures shows both fluorescently labeled lipids and on the right side in the figures the channel for the LUV dye is shown. All highly tensed GUV-membranes reveal a high amount of docked LUVs. The freestanding part of the GUV-membrane contains many fluorescence peak intensities that refer to docked LUVs or clusters of docked LUVs. At the adhesion site of the GUVs the fluorescence intensity of the LUV dye was detected in all GUVs 14-21. The fluorescence intensity at the adhesion site is indicative for the diffusion of LUV dye into this region after fusion LUV with the GUV-membrane occurred. To prove, that the fluorescently labeled dye originating from LUVs can diffuse freely in the GUV-membrane FRAP experiments were done at the adhesion site which is described in experiment 4 .

In the figure 5.8 the LUV dye A594 diffused into the connection site between the two GUVs 21 and 22 that adhered on the substrate surface in near vicinity to each other. The membrane tension of $1.5 \mathrm{mN} / \mathrm{m}$ for GUV 21 and $2.3 \mathrm{mN} / \mathrm{m}$ for GUV 22 (Figure $5.8 \mathrm{D}$ ) resulted in higher fusion efficiency of LUVs detected through an increased fluorescence intensity of the LUV dye A594 at the GUV-membrane. An area scan from figure 5.8 $\mathrm{A}$ is shown in the graph $(\mathrm{C})$ which shows the fluorescence intensity of the GUV dye A390 and the LUV dye A594. The docked LUVs on both GUV- membranes should be blocked to diffuse into the connection site of the two GUVs. Therefore the fluorescence intensity of the LUV dye A594 originates from LUVs that fully fused with the GUV-membrane. The mean fluorescence intensity of the LUV dye A594 is increased at both GUVs. Especially, at the connection site the fluorescence intensity is higher which could be due to the presence of two lipid bilayers containing the fluorescently labeled lipid originating from fused LUVs. The error for the measurement of the vesicle radii for the GUVs 21 and 22 is assumed to be slightly higher because of the non-spherical shape of the GUVs.

In summary, vesicle docking and fusion to GUVs bearing an elevated membrane tension is increased which was detected through a fluorescence intensity increase of the LUV dye at the GUV-adhesion site and at the connection site between two GUVs adhering in near vicinity on the substrate surface. 

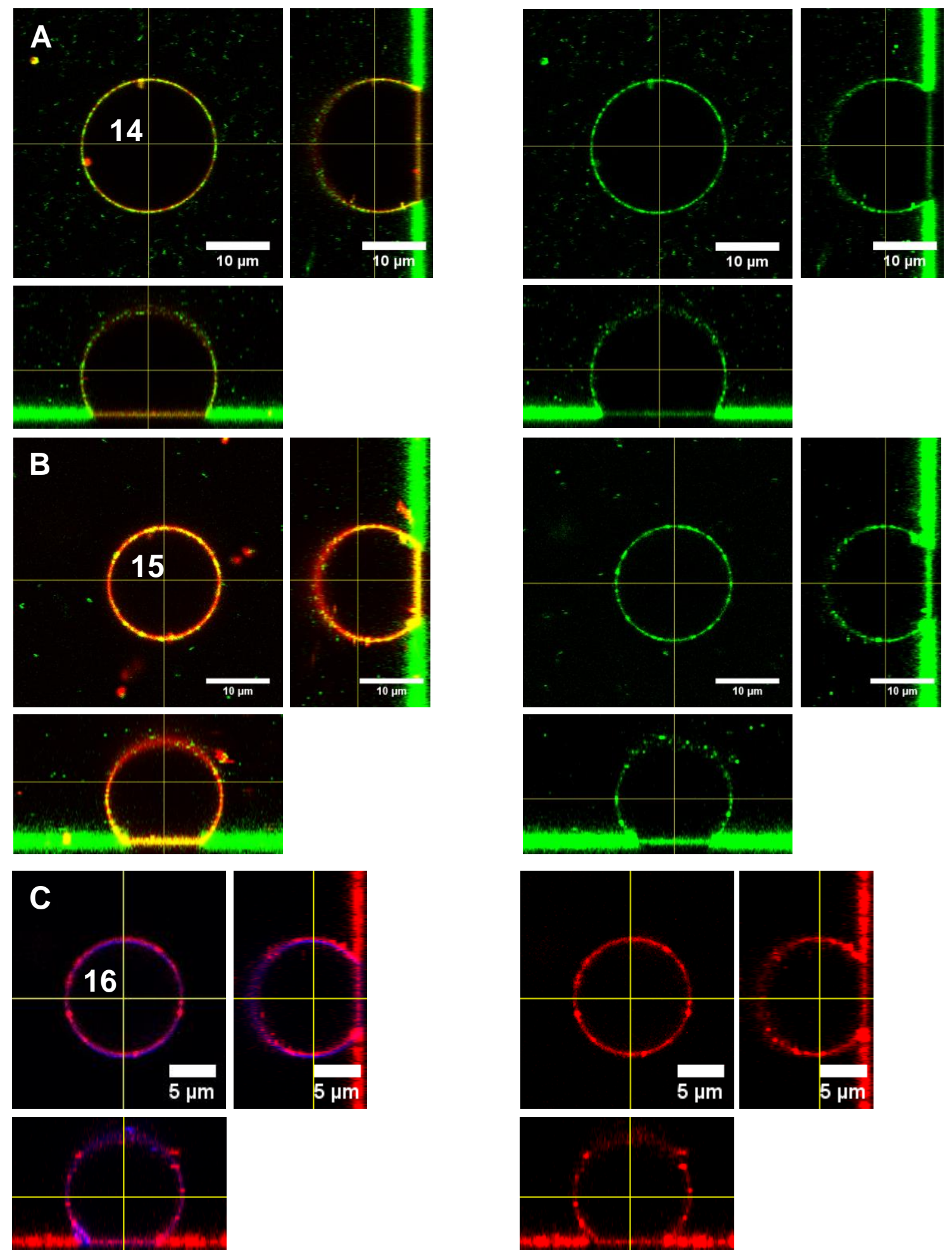

\begin{tabular}{|c|c|c|c|c|}
\hline GUV No & $R_{\mathrm{i}} / \mu \mathrm{m}$ & $\widetilde{R}_{\mathrm{v}} / \mu \mathrm{m}$ & $\Delta A / A_{0} / \%$ & $\tau / \mathrm{mN} \mathrm{m}^{-1}$ \\
\hline 14 & 8.8 & 10.6 & 3.9 & 8.6 \\
\hline 15 & 6.5 & 9.0 & 2.1 & 4.0 \\
\hline 16 & 4.1 & 6.1 & 1.4 & 2.4 \\
\hline
\end{tabular}

Figure 5.6. Strongly adhered GUVs with high membrane tension reveal LUV fusion. Fluorescently labeled lipids in the GUV/LUV-membrane: TR (red) / A488 (green), A390 (blue) / A594 (red). A)-C) Cross-sectional images of GUVs reveal high amount of docked and fused LUVs at the GUV-membrane. D) The table shows the measured radii and area changes of the GUVs14-16 and the calculated membrane tension. 

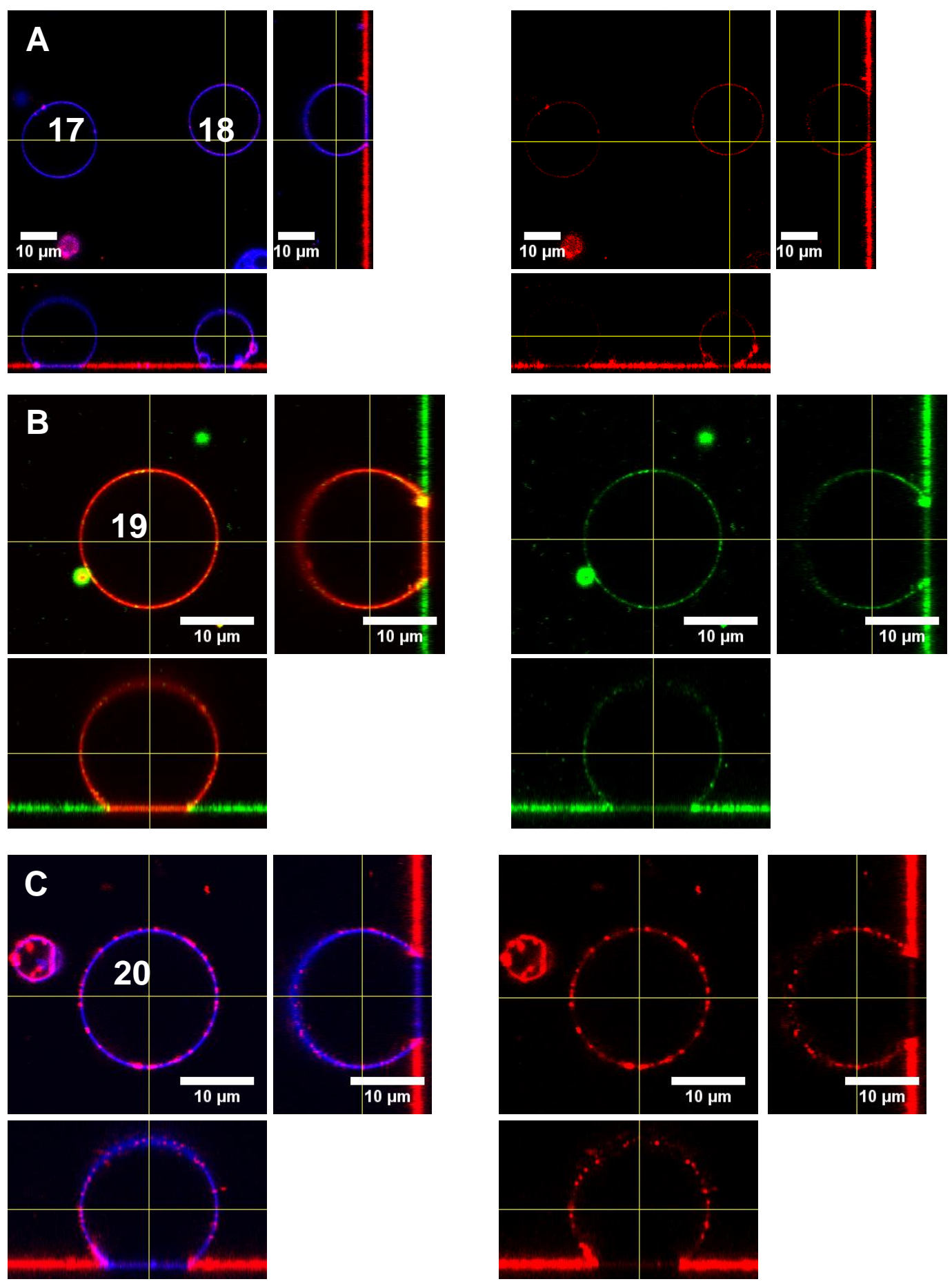

D \begin{tabular}{|c|c|c|c|c|}
\hline GUV No. & $R_{\mathrm{i}} / \mu \mathrm{m}$ & $\widetilde{R}_{\mathrm{v}} / \mu \mathrm{m}$ & $\Delta A / A_{0} / \%$ & $\tau / \mathrm{mN} \mathrm{m}^{-1}$ \\
\hline 17 & 6.0 & 10.2 & 0.83 & 1.1 \\
\hline 18 & 7.3 & 9.7 & 2.5 & 5.0 \\
\hline 19 & 5.9 & 9.2 & 1.1 & 1.7 \\
\hline 20 & 5.8 & 9.2 & 1.1 & 1.7 \\
\hline
\end{tabular}

Figure 5.7. Strongly adhered GUVs with high membrane tension reveal LUV fusion (right). Fluorescently labeled lipids in the GUV/LUV-membrane: TR (red) / A488 (green), A390 (blue) / A594 (red). A)-C) Cross-sectional images of GUVs reveal high amount of docked and fused LUVs at the GUVmembrane. D) The table shows the measured radii and area changes of the GUVs14-16 and the calculated membrane tension. 

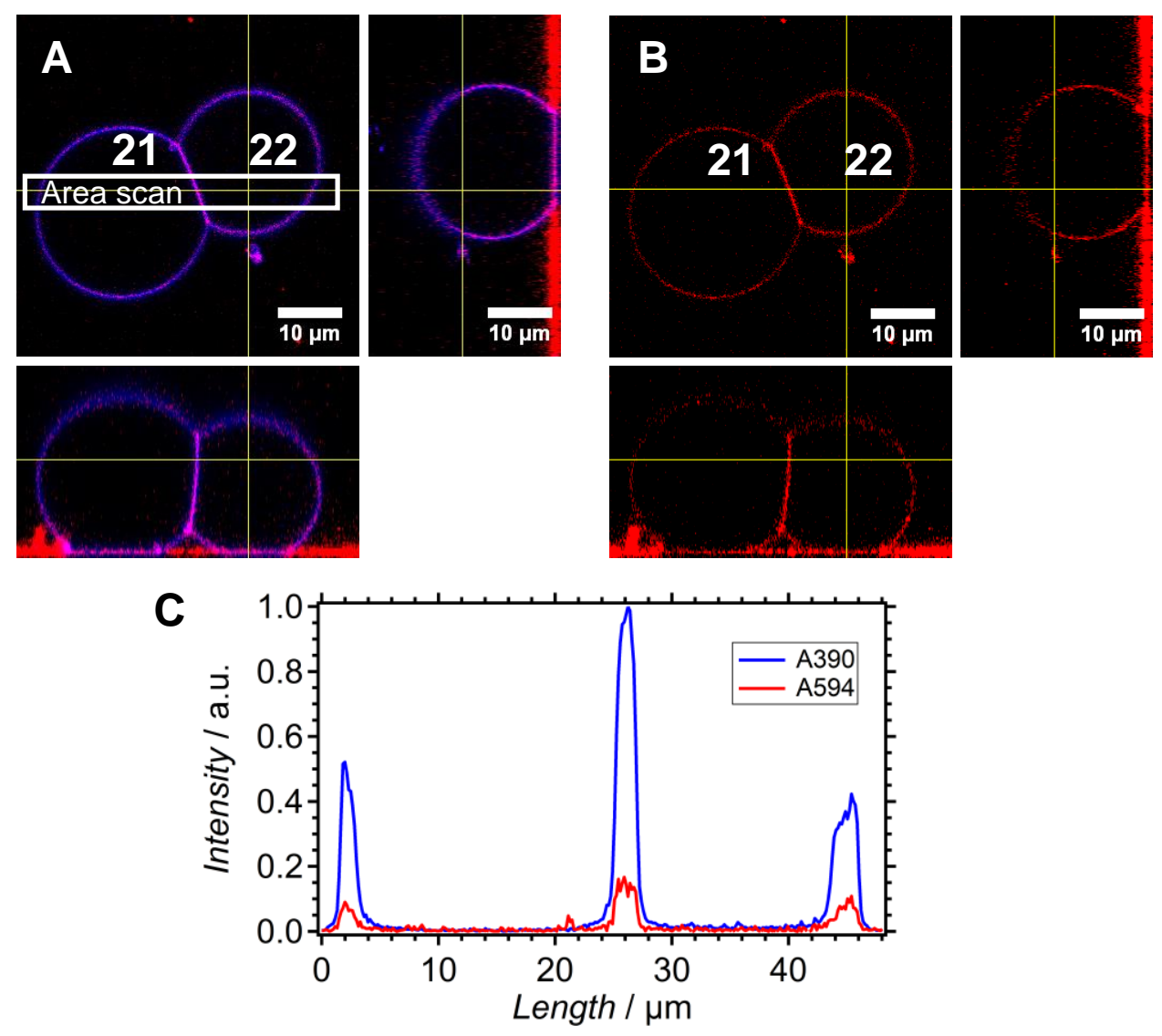

D

\begin{tabular}{|c|c|c|c|c|}
\hline GUV No. & $R_{\mathrm{i}} / \mu \mathrm{m}$ & $\widetilde{R}_{\mathrm{v}} / \mu \mathrm{m}$ & $\Delta A / A_{0} / \%$ & $\tau / \mathrm{mN} \mathrm{m}^{-1}$ \\
\hline 21 & 8.1 & 13.2 & 1.0 & 1.5 \\
\hline 22 & 7.5 & 11.6 & 1.4 & 2.3 \\
\hline
\end{tabular}

Figure 5.8. Connected GUVs adhering in near vicinity on the substrate. A) The two channel image shows the two GUVs (blue) and the area scan in the white box. B) The red channel of the LUV dye A594 shows a fluorescence intensity all over the GUV membrane. C) The graph represents the area scan from (A) for the mean fluorescence intensity of both lipid dyes. The LUVs fused with the GUVmembrane and thus the LUV dye is present at the connection site between the LUVs. D) The table lists the measured radii, area changes and corresponding membrane tensions. 


\subsubsection{Experiment 3: Time-dependent vesicle fusion}

Notably, vesicle fusion assays described in the literature are time dependent. HERNANDEZ et al. showed that the membrane fusion rate promoted by SNARE-proteins on larger vesicles $(40-70 \mathrm{~nm})$ is significantly lower than the fusion rate between small vesicles $(20-30 \mathrm{~nm}) .{ }^{9} \mathrm{In}$ the presented samples, much larger vesicles were used to investigate membrane fusion as a function of lateral membrane tension: The GUV size ranged from the radii $\widetilde{R}_{\mathrm{v}}=4.3 \mu \mathrm{m}$ up to $18.7 \mu \mathrm{m}$, whereas the average LUV size was measured around $100 \mathrm{~nm}$. Therefore, it was inevitable to measure the docking and fusion of LUVs to a tensed GUV-membrane over time. In figure 5.9 a cross-sectional view of a GUV with a membrane tension of $2.0 \mathrm{mN} / \mathrm{m}$ is depicted three minutes after the LUV addition and after 13 minutes in
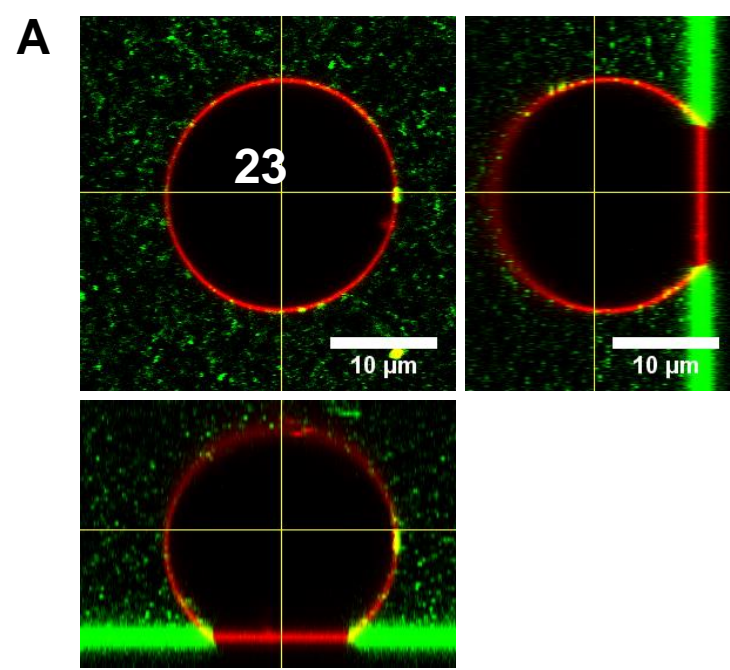

B
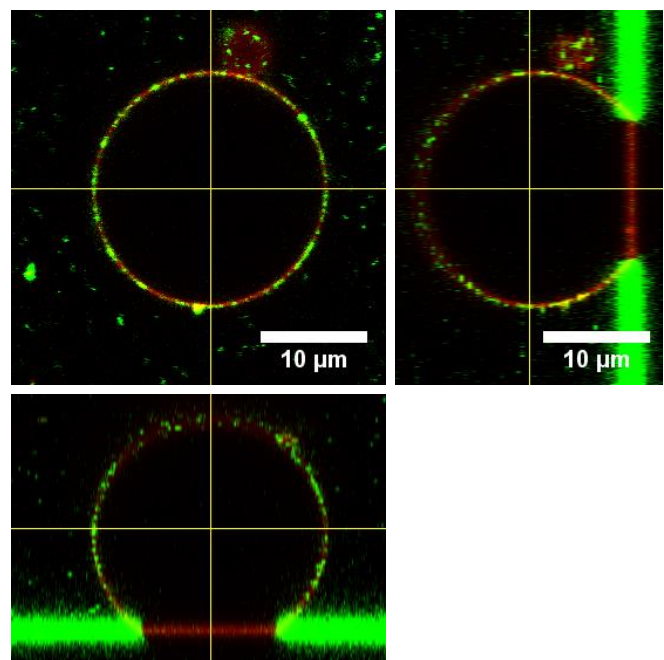

C

\begin{tabular}{|c|c|c|c|c|}
\hline GUV No. & $R_{\mathrm{i}} / \mu \mathrm{m}$ & $\widetilde{R}_{\mathrm{v}} / \mu \mathrm{m}$ & $\Delta A / A_{0} / \%$ & $\tau / \mathrm{mN} \mathrm{m}^{-1}$ \\
\hline 23 & 7.0 & 10.8 & 1.2 & 2.0 \\
\hline
\end{tabular}

Figure 5.9. Fusion between a GUV and LUVs as a function of time and tension. The two channel images for both lipid dyes is shown on the right. On the left, the images shows the fluorescence intensity originating from LUVs. A) The adhered GUV (red) was imaged directly after LUV addition (green). The LUVs float around the GUV and dock to the GUV-membrane. B) After 10 minutes many LUVs (green) docked to the GUV-membrane (red). The LUV dye is distributed all over the GUV-membrane. C) The table lists the measured radii, calculated area change and membrane tension of the depicted GUV. 
figure $5.9 \mathrm{~B}$. On the right side of the figure 5.9 the green channel for the LUV dye A488 is depicted. In figure $5.9 \mathrm{~A}$, directly after the addition of LUVs, only a few LUVs (green) dock to the GUV-membrane (red) whereas after 13 minutes much more LUVs dock to the GUV-membrane. In figure $5.9 \mathrm{~B}$ the adhesion area of the GUV-membranes shows nearly no fluorescence intensity whereas in figure $5.9 \mathrm{~B}$ the adhesion area reveals a low fluorescence intensity of the dye A488 originating from the LUVs that fused with the GUV-membrane. To sum up, the docking and fusion of the LUVs to a tensed GUVmembrane can be measured over time. The fusion rate of vesicles, investigated by HERNANDEZ et al., decreased significantly whereas the results of the described experiment in figure 5.9 indicates that an elevated membrane tension increases the amount of docking and fusion between vesicles of different sizes significantly. 


\subsubsection{Experiment 4: FRAP measurements at the adhesion site of GUVs}

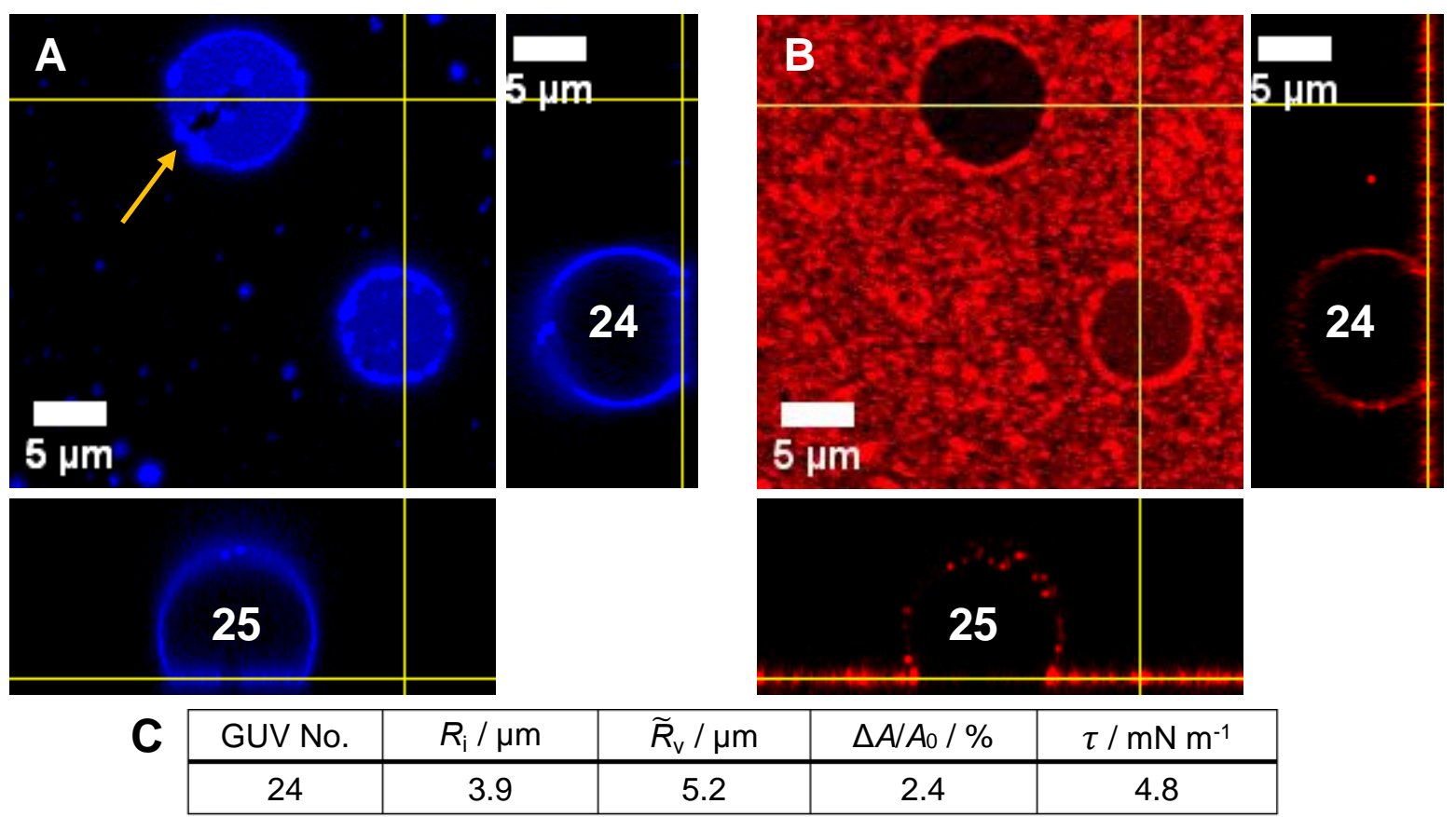

Figure 5.10. Comparison of tensed and ruptured GUV-membranes. A) The GUV-membrane adhesion area of both GUVs is shown in the $x y$-plane. GUV 25 ruptured at the adhesion site. B) LUV docking and fusion appeared on GUV 4 more frequently because of the high fluorescent intensity originating from the LUV dye A594. C) The table represents the measured and calculated values for GUV 24.

Fortunately, it was possible to measure a highly tensed GUV and a GUV with a ruptured membrane in one sample to compare the amount of fusion and docking through the fluorescence intensities for the LUV dye at the GUV-membrane (Figure 5.10). GUV 25 in figure 5.10 had a ruptured membrane at the adhesion site whereas GUV 24 was stable at a membrane tension of $4.8 \mathrm{mN} / \mathrm{m}$. Somehow, GUV 25 with the ruptured membrane on the substrate did not spread by forming a supported lipid bilayers on the substrate surface. A direct comparison of a highly tensed membrane on GUV 24 with the ruptured membrane of GUV 24 reveals that the fluorescence intensity originating from the LUV dye A594 is higher at the tensed GUV 24. During the process of adhesion the membrane of GUV 25 ruptured at the adhesion site. This membrane rupture could have occurred after the addition of LUVs to the sample because a few LUVs dock to the GUV 25 bearing a low membrane tension. LUV docking to the tensed membrane of GUV 25 could have occurred before the rupture event. However, this experiments is a good example for the increased fusion efficiency on a tensed GUV-membrane compared to a GUVmembrane with a low membrane tension.

A FRAP-measurement in figure 5.11 shows that the fluorescence intensity of the LUV dye A594 on the adhesion site of the GUVs has higher intensity at the GUV 24 compared to the GUV 25. The lower fluorescence intensity at the adhesion site of GUV 25 was a result or the low amount of fused LUVs that relates to the low membrane tension. Bleaching of the membrane at the adhesion site of GUV 24 leads to a finished recovery after seven seconds with a diffusion coefficient of around $1.3 \mu \mathrm{m}^{2} \mathrm{~s}^{-1}$. The high 
immobile fraction of around $40 \%$ could be a result of the strong bleaching effect of the LASER through the GUV-membrane.

The aspect that LUVs adhere on the surface could lead to the assumption that a lipid bilayer is formed on the substrate surface through spreading of the LUVs. In figure $5.11(\mathrm{E}-\mathrm{G})$ the FRAP-measurement of adhered LUVs on the surface is shown. Bleaching of adhered LUVs on the substrate surface yields no fluorescence recovery. With this FRAP-measurement it is proven that the adhered LUVs did not spread on the substrate surface and therefore a possibly spread lipid bilayer on the substrate surface could not interfere with the adhered GUV-membranes.

In summary, the highly tensed GUV 24 exhibited a much higher amount of docking and fusion for LUV which was proven by the FRAP-experiment. A ruptured membrane on GUV 25 with a low membrane tension in the same sample served as an intrinsic blind sample that revealed a lower amount of docked and fused LUVs.

LUV fusion the GUV-membrane was proven by a FRAP-measurement at the adhesion area of the GUV. In figure 5.12 a FRAP-measurement on the adhesion site of GUV 14 from figure 5.6 A is shown. Compared to the adhered LUVs, surrounding the adhesion site of the GUV on the glass surface, the adhesion area contained a lower fluorescence intensity. Therefore, the brightness of the FRAP-images was increased to visualize the bleaching and recovery on the adhered GUV-membrane. Bleaching with a LASER $(488 \mathrm{~nm})$ led to a loss of fluorescence intensity at the green ROI shows. Ten seconds after the bleaching the recovery reached $60 \%$ of the initial fluorescence intensity. For the fluorescently labeled dye A488 at the adhesion site a diffusion coefficient of $0.8 \mu \mathrm{m}^{2} \mathrm{~s}^{-1}$ was calculated and an immobile fraction of about $17 \%$ was measured. With this FRAP-measurement it is proven that the LUVs fused with the GUV membrane and the lipid dye A488, originating from the LUVs, diffused into the adhesion area of the GUV-membrane.

In figure 5.13 the edge of the adhesion area was bleached and a recovery was detected whereby the fluorescence intensity recovered to around $60 \%$ to the initial value. The bleaching effect of the LASER to the fluorescently labeled lipids in the GUV membrane could be responsible to the lowered fluorescence intensity at the bleached region in combination with the effect of an immobile fraction of the lipids. The non-spherical geometry of the GUV is a result of the connection to the other GUV adhering on the substrate in near vicinity. Therefore, the calculated membrane tension comprises a larger error because in theory the GUVs are assumed to be spherical omitting a spherical cap due to the adhesion on the surface. A precise measurement of the diffusion coefficient was not possible but fluorescence recovery proved that the lipids of the LUVs a part of the highly tensed GUV-membrane.

In summary, the FRAP-measurements shown in the figures 5.11 and 5.12 proved lipid mixing between the adhered GUV and LUVs, and the LUVs do not spread on the surface by forming a lipid bilayer that could possibly fuse with the adhered GUV membrane through edge-fusion. 

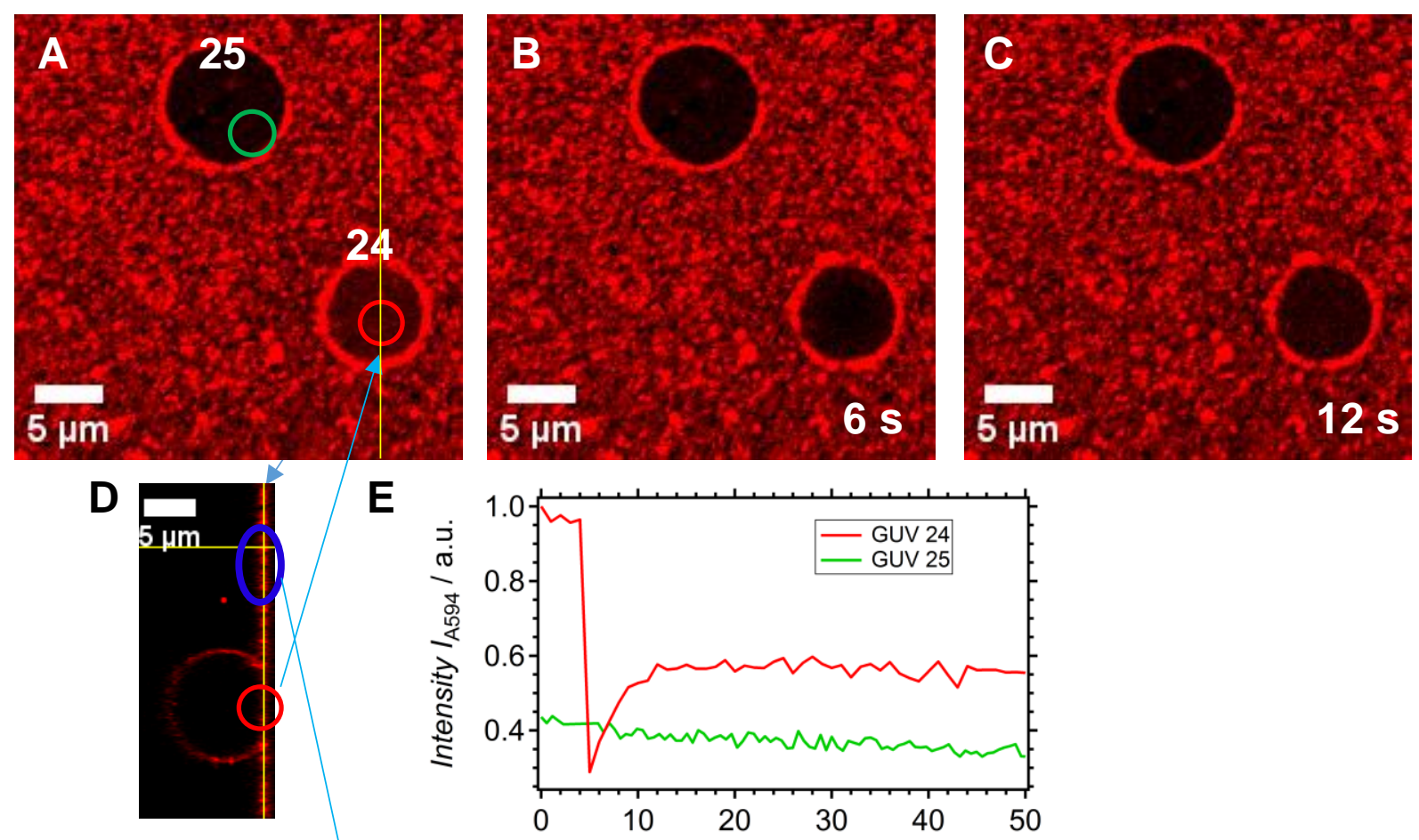

$\mathbf{E}$
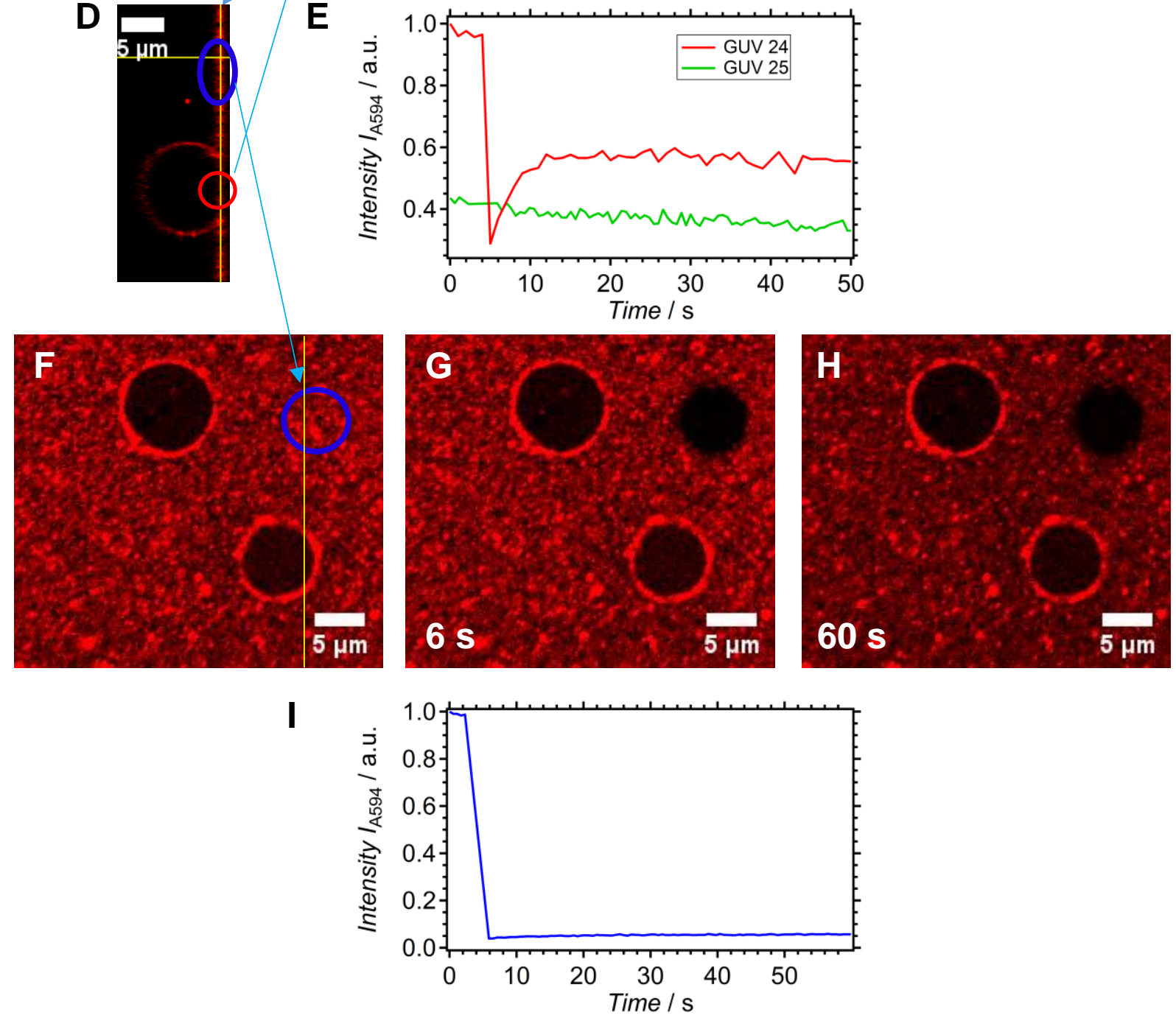

Figure 5.11. FRAP-measurement at the adhesion site of the GUVs from figure 5.25. A) The red circle shows the area for the bleaching on the GUV 24 with the LASER $(561 \mathrm{~nm})$ and the green circle the reference area on GUV 25. B) The image was taken directly after the bleaching of the red circle area depicted in (A). C) Seven seconds after bleaching the recovery was finished but with around $40 \%$ of immobile fraction. D) The cross-sectional ( $y z$-plane) image shows bleaching areas on the substrate surface. E) The graph shows the recovery of the mean fluorescence intensity at the red ROI of GUV 24. F)-H) The bleached LUVs on the surface shows no recovery after $60 \mathrm{~s}$. I) The graph plots the fluorescence intensity of the blue ROI 

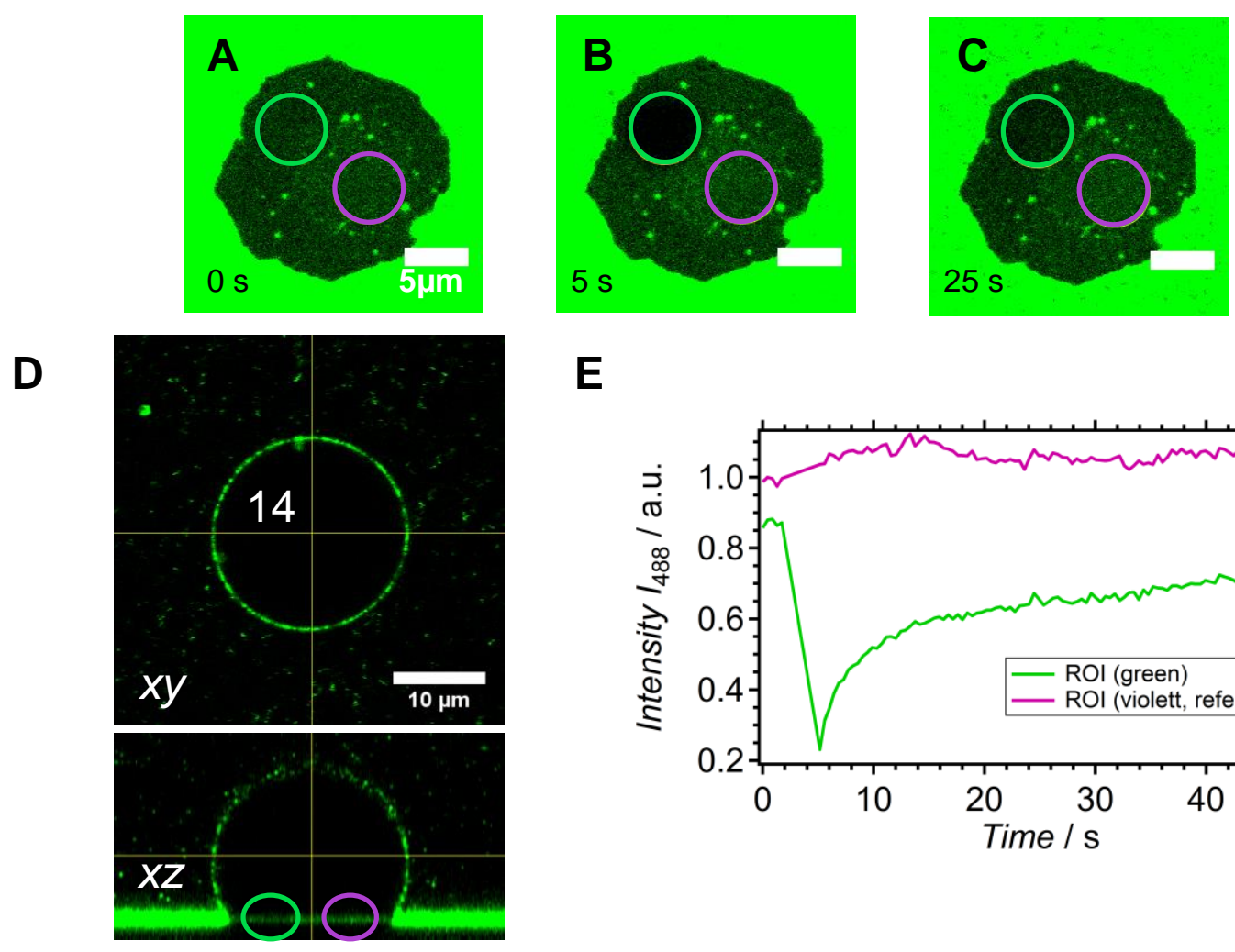

$\mathbf{E}$
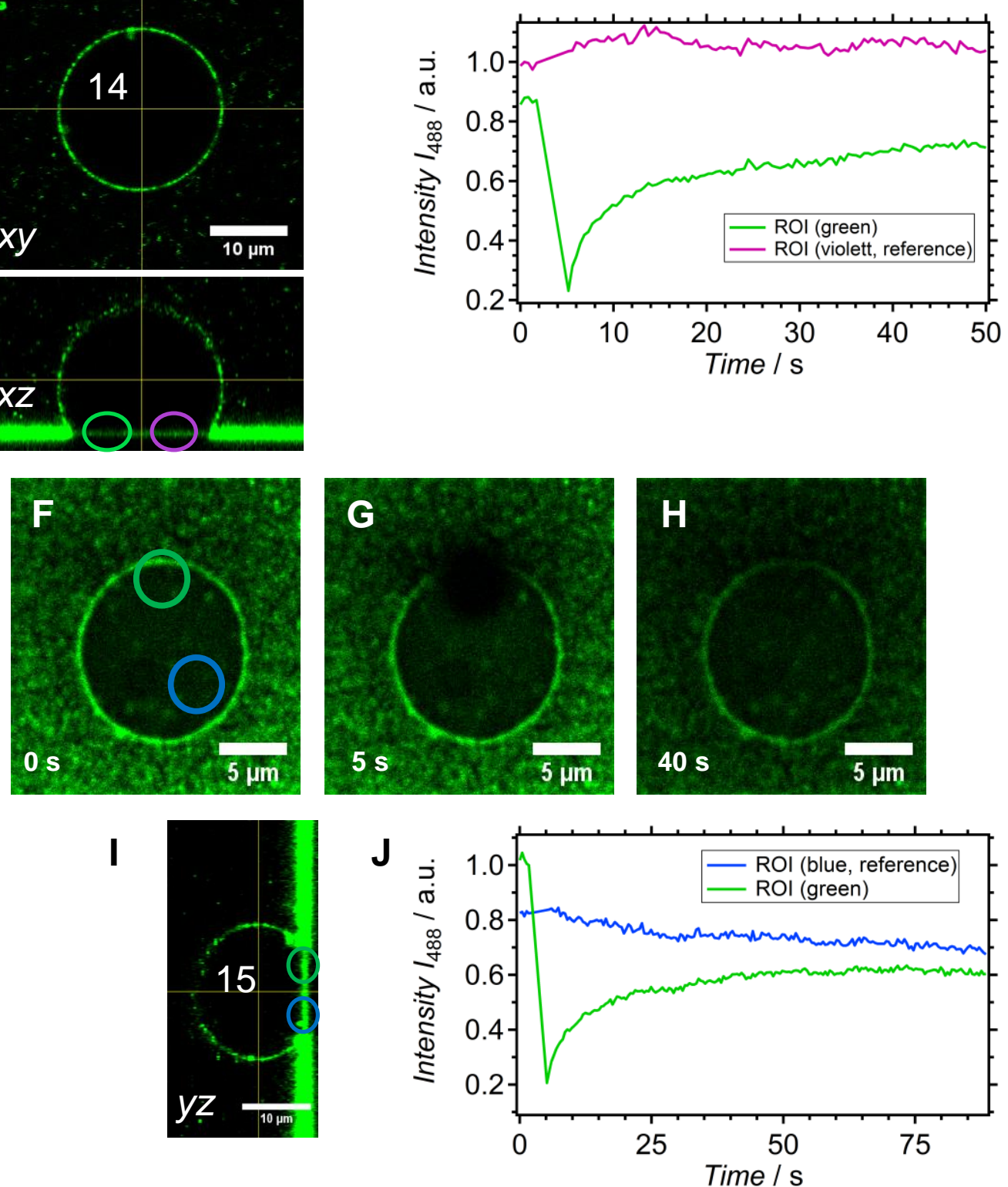

Figure 5.12. FRAP measurement on the adhesion area of the GUV 14 depicted in figure $5.6 \mathrm{~A}$. The fluorescence intensity brightness in the images $(A)$ - $(C)$ was increased to visualize the fluorescence intensity before (A), after bleaching (B) and the recovery (C). D) The cross-sectional view shows the bleaching (green circle) and reference area (violet circle). E) The graph shows the mean fluorescence intensity for both circles with the violet circle as a reference (100\%). F)-H) Images of a FRAPmeasurement at the edge of the adhered membrane of GUV 15. G) The edge of the adhered GUVmembrane is bleached. $\mathrm{H}$ ) The fluorescently labeled lipid A488 recovers to $60 \%$ of its initial intensity. I) The green ROI in the cross-sectional view of GUV 15 shows the bleaching area and the blue ROI the reference area of this FRAP-measurement. E) The mean fluorescence intensity of the bleached ROI and reference $\mathrm{ROI}$ area is plotted in the graph. 

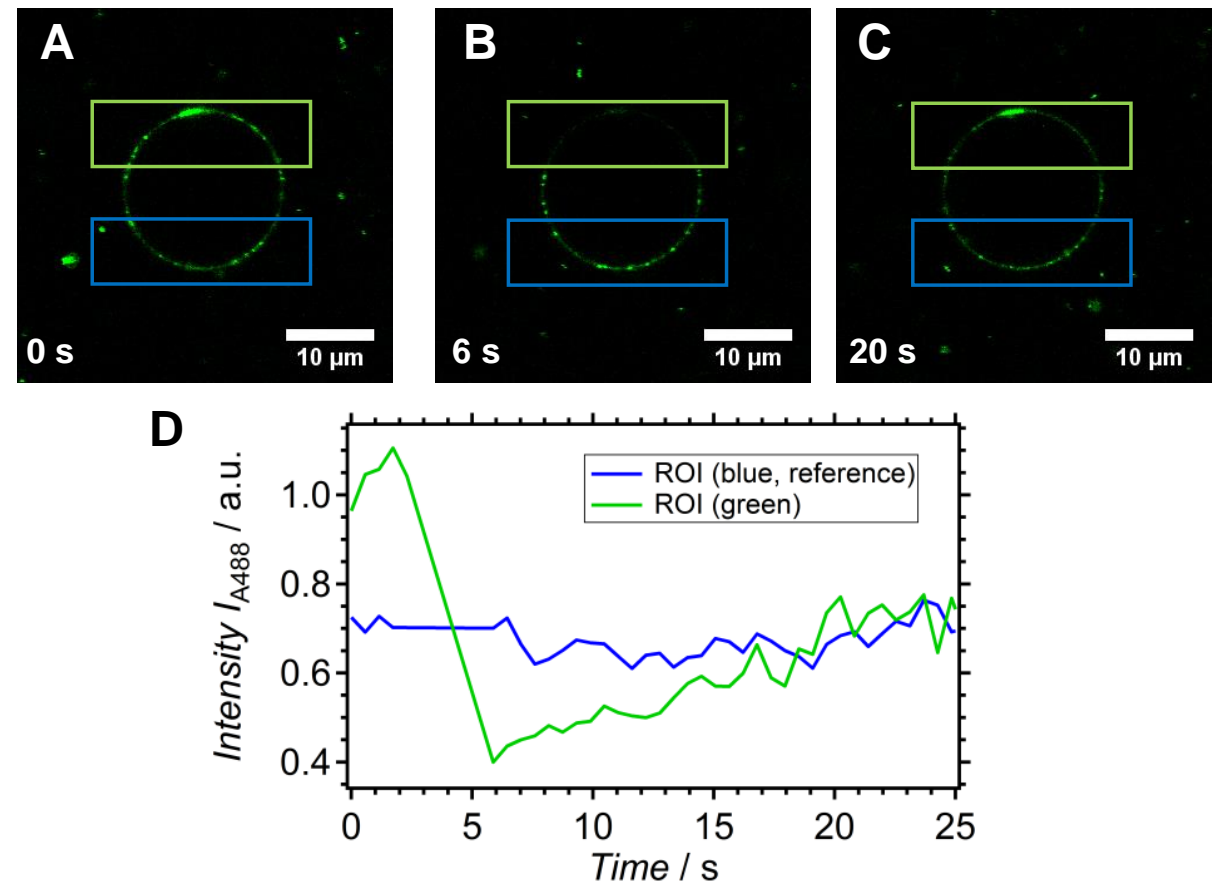

Figure 5.13. FRAP of docked LUVs on the GUV 15 from figure 5.6 B. A) The docked LUVs were imaged with the LASER for the dye A488. The green square shows the area that was bleached by the Laser in (B). The blue square served as a reference area for the measurement of the fluorescence intensity of the dye A488 plotted in (D). C) A recovery of fluorescence intesity occurred.

The bright fluorescence of the LUV dye at the GUV-membrane raised the question whether the docked LUVs can freely diffuse on the fluid GUV-membrane. In figure 5.13 the FRAP measurement at the freestanding membrane of GUV 15 with a membrane tension of $4.0 \mathrm{mN} / \mathrm{m}$ is shown. Bleaching of the LUV dye A488 in the green ROI (Figure $5.13 \mathrm{~B}$ ) leads to a loss of fluorescence intensity. The bleaching and recovery of the LUV dye is plotted in the graph (D). The blue ROI at the other side of the freestanding GUV-membrane served as a reference measurement. The initial fluorescence intensity between the two ROls were differently which is a result of an unequal distribution of LUVs at the surface of the GUVmembrane. After bleaching of the LUV dye (B) the fluorescence intensity recovers to around $70 \%$ of its initial value. This FRAP-measurement reveals that diffusion of LUVs on the GUV surface occurs at the freestanding membrane. 


\subsubsection{Content mixing of LUVs with adhered GUVs}

The ultimate proof for imaging of two bilayers in the context of vesicle-vesicle fusion is content mixing. The content mixing experiment, illustrated in figure 5.7, was done by adding LUVs, containing the water soluble dye $A T T O^{\circledR} 488$, to the sample with the adhered GUVs. An increased fluorescence intensity inside the GUV reveals that the LUVs fuse fully with the GUV-membrane. Merging of vesicle membranes is mediated by the use of SNARE proteins on both vesicles. The LUVs contained synaptobrevin, and the GUVs the $\Delta N 49$-complex.

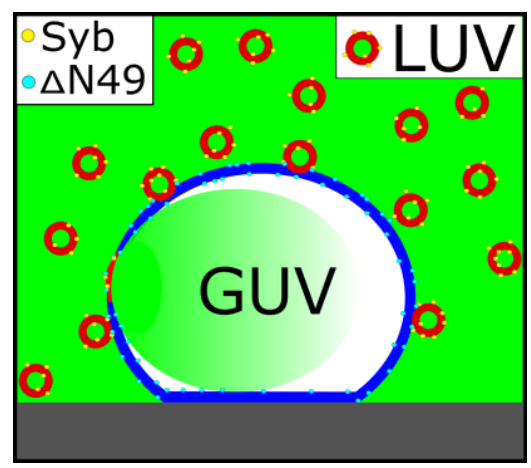

Figure 5.14. Mixing of contents was achieved by the fusion of LUVs to the adhered GUV so that the inside of the LUVs mixed with the GUV solution inside and the green fluorescent dye diffused into the GUV. Thus the green fluorescence intensity inside the GUV should increase.

In figure 5.15 three GUVs with different sizes and adhesion areas are shown in a $x y$-plane picture and a corresponding cross-sectional view on the right and bottom. After the incubation of LUVs containing the water soluble green fluorescent dye at the inside of the LUVs it was detected that the GUVs 27 and 28 show a high fluorescence intensity compared to GUV 26 with nearly no intensity emitted from the inside (figure $5.15 \mathrm{C}+\mathrm{D}$ ). The result for the calculated membrane tension on each GUV and the corresponding green fluorescence intensity inside the GUV is given in the table 5.1. The surrounding solution served as a reference for the fluorescence intensity $l_{488}$ inside the GUVs.

A small adhesion area of GUV 26 generated a low membrane tension of $0.12 \mathrm{mN} / \mathrm{m}$. The larger adhesion area compared to the vesicle radius on GUV 27 and 28 resulted in a larger membrane tension of $0.29 \mathrm{mN} / \mathrm{m}$ for GUV 27 and $0.94 \mathrm{mN} / \mathrm{m}$ for GUV 28. Interestingly, the fluorescence intensity originating from the LUV content dye ATTO ${ }^{\circledR} 488$ increased inside the GUVs 27 and 28 up to $60 \%$ and respectively $100 \%$ of the fluorescence intensity in the surrounding solution whereas GUV 26 with a low membrane tension showed no significant fluorescence intensity increase (Table 5.1). The smallest GUV28 with the highest membrane tension contained the same fluorescence intensity inside the GUV as the surrounding buffer solution. 

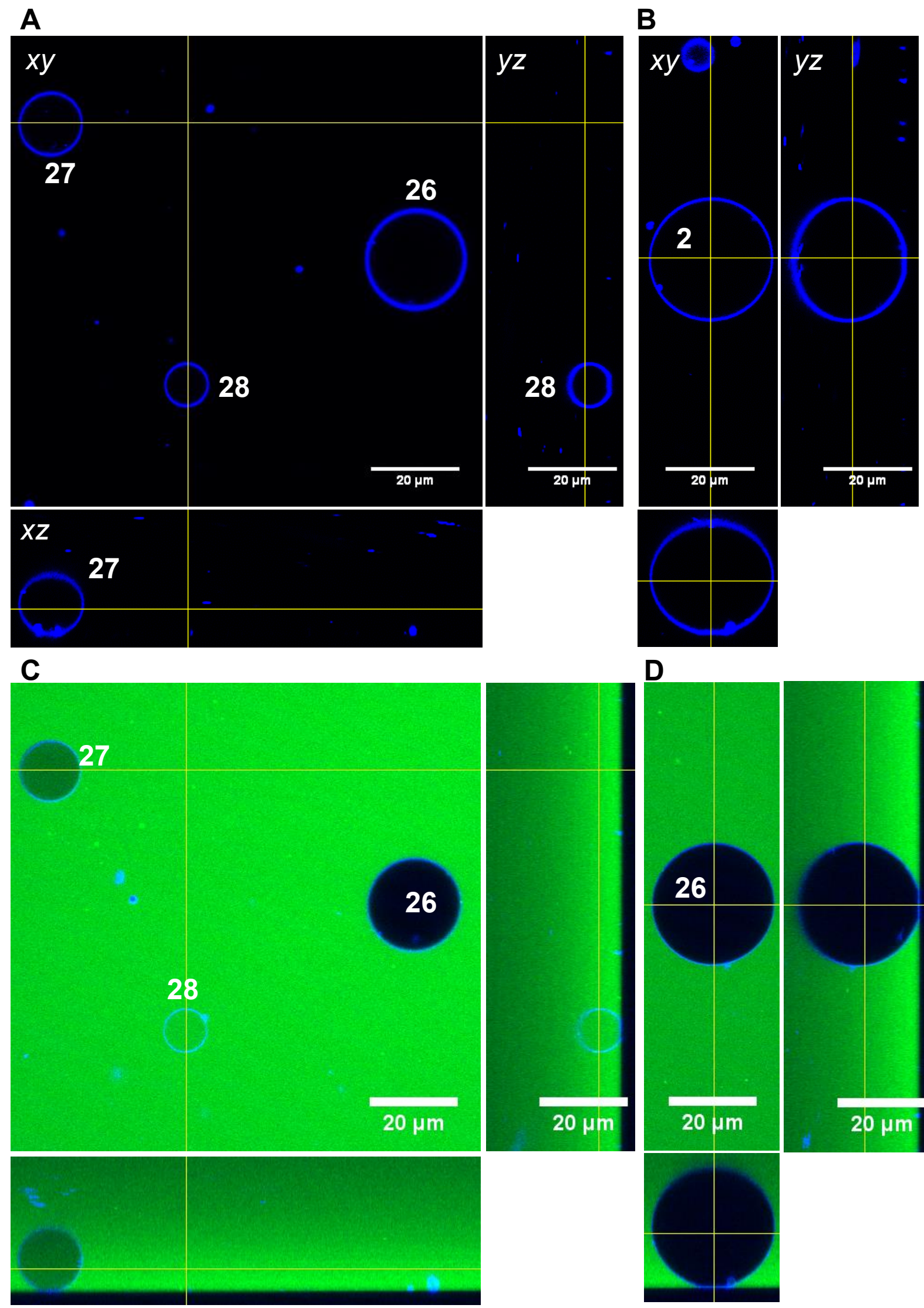

Figure 5.15. Three GUVs slightly adhered on the glass surface of the sample. A) Three GUVs are depicted in on image $x y$-image plane. B) The cross-sectional image for GUV 26 is shown. C) After LUV incubation GUVs 27 and 28 contained the green fluorescent dye ATTO ${ }^{\circledR} 488$. D) The largest GUV with the smallest membrane tension shows no fluorescence intensity from the LUV dye 
Table 5.1. The contact radius $R \mathrm{i}$ and the vesicle radius $\widetilde{R}_{\mathrm{v}}$ was measured for each GUV. With these measured values for each GUV the area change $\triangle \mathrm{A} / \mathrm{A} 0$ and the corresponding membrane tension was calculated that can be compared to the fluorescence intensity of the LUV content dye that was measured at the inside of the GUV after LUV fusion possibly occurred. A comparison of the LUV volume to the GUV volume reveals how many time the GUVs are greater than an average LUV with a diameter of $200 \mathrm{~nm}$.

\begin{tabular}{|c|c|c|c|c|c|c|}
\hline GUV No. & $R_{\mathrm{i}} / \mu \mathrm{m}$ & $\widetilde{R}_{\mathrm{V}} / \mu \mathrm{m}$ & $\Delta A / A_{0} / \%$ & $\tau / \mathrm{mN} \mathrm{m}^{-1}$ & $I_{488} / \%$ & $\bar{V}_{\text {LUV }} / V_{\mathrm{GUV}}$ \\
\hline 26 & 4.30 & 13.98 & 0.05 & 0.12 & 6.6 & $1 /\left(2.7 \times 10^{6}\right)$ \\
\hline 27 & 3.35 & 7.31 & 0.28 & 0.29 & 60 & $1 /\left(3.9 \times 10^{5}\right)$ \\
\hline 28 & 2.88 & 5.01 & 0.73 & 0.94 & 100 & $1 /\left(1.3 \times 10^{5}\right)$ \\
\hline
\end{tabular}

The three GUVs $26-28$ in figure 5.15 contained different volumes. Therefore, the dilution of the dissolved LUV content dye could affect the measurement for the fluorescence intensity inside the GUVs. The difference in dilution between the GUVs can be compared by the LUV-volume $\bar{V}_{\text {LUV }}$ with the three the GUV-volumes $V_{\text {GUV }}$. The mean size of the LUVs can be assumed to be around $200 \mathrm{~nm}$ in diameter containing a mean volume $\bar{V}_{\text {LUV }}=4.2 \times 10^{-3} \mu \mathrm{m}^{3}$. The mean volume $\bar{V}_{\text {LUV }}$ of the LUVs is compared to the three GUVs 26-28 in table 5.1. The largest GUV 26 compared to the smallest GUV 28 contains a volume that is one order of magnitude larger which implies that the dilution could play a central role to the measured fluorescence intensity at the inside of each GUV.

A FRAP-measurement, shown in figure 5.16, shows that the GUV content was bleached by the LASER and thus, content mixing of LUVs to the GUV 28 was proven. The fluorescence intensity in the GUV before the bleaching with the LASER was lower compared to figure 5.15 because of the scanning LASER of the microscope. However, the graph in figure 5.16 D plots the fluorescence intensity inside
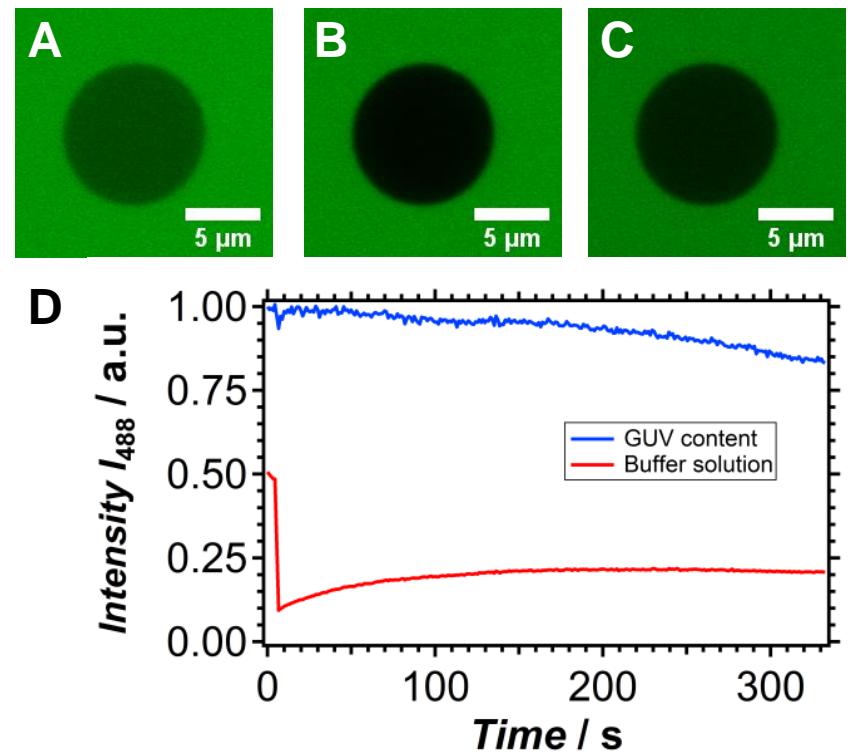

Figure 5.16. FRAP measurement of GUV 3 with on a longer time scale. The images were taken before (A) and after (B) bleaching of the GUV content at $6 \mathrm{~s}$. Image (C) was the last one $324 \mathrm{~s}$ after the GUV content was bleached. D) The graph outlines the green fluorescence intensity inside the GUV and in the buffer solution as a reference. A slightly increase of the green dye in the GUV was measured during time which levels off at around $160 \mathrm{~s}$ at an intensity of $0.22 \mathrm{a}$.u. 
the GUV as a function of time from the FRAP-measurement. Interestingly, the fluorescence intensity slightly increased after the bleaching which indicates that LUVs release their content into the GUV through membrane fusion. To exclude a diffusion of the dye ATTO $^{\circledR} 488$ from the surrounding buffer solution into the GUV a blind sample without SNAREs in figure 5.17 is depicted. Even strongly adhered GUVs with a high membrane tension do not contain any fluorescence intensity so that a diffusion of the water soluble LUV dye through the GUV-membrane is disproved.

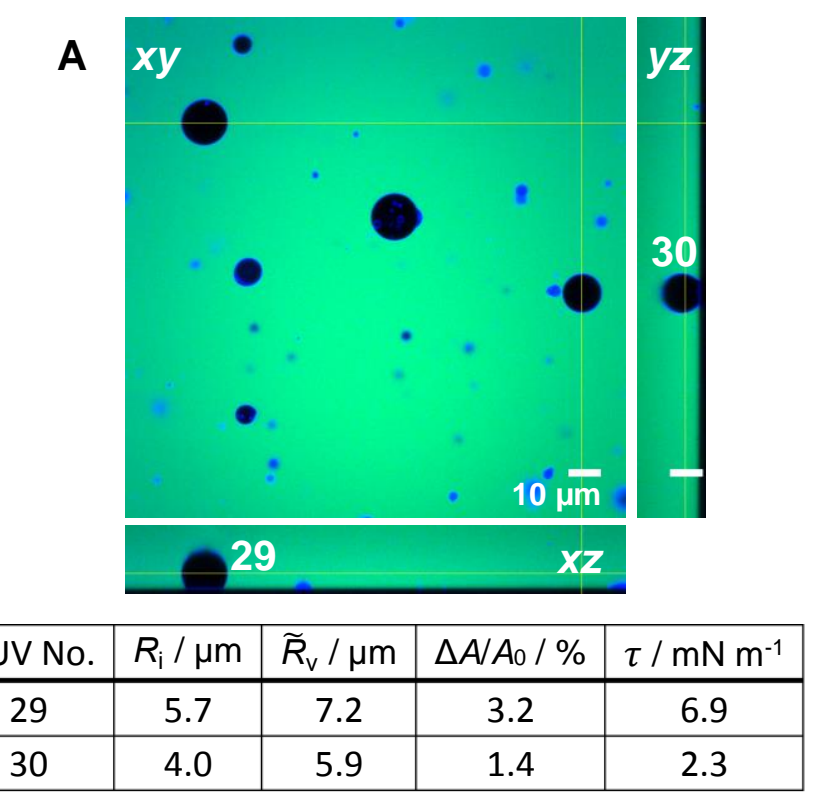

Figure 5.17. A) A blind sample without SNAREs shows GUVs after incubation of LUVs but without any diffusion of the green fluorescent dye into the content of the GUVs. LUV fusion or diffusion of the green dye from the buffer solution into the GUVs did not occur. The cross-sectional view shows that the GUVs strongly adhere on the surface. B) The table lists the measure radii, area changes and the calculated membrane tension of the GUVs 29 and 30.

For the two GUVs 29 and 30 from figure 5.17 the calculated membrane tension is listed in the table (B). In this blind sample no content mixing was detected although the membrane tensions of the GUVs occurred to be much higher compared to the GUVs 27 and 28.

Summing up the results of this chapter, the content mixing experiment proved that full fusion of the two lipid bilayers between LUVs and tensed GUVs is achieved by using SNAREs as fusion promoters. 


\subsection{Membrane fusion on stretched SLBs}

The first step for the investigation of membrane fusion on a supported lipid bilayer (SLB) was to achieve area dilatation of the SLBs on an extendable substrate. Previously stretching and compression of lipid bilayers was investigated and established by Staykova et al. They utilized a thin sheet of polydimethylsiloxane (PDMS) as a possible substrate for the dilatation of SLBs. ${ }^{58-59}$ This seems a rather simple approach for the investigation of membrane area change due to the stretching of the PDMS surface but exactly that stretching of the PDMS surface is limited to the extendibility of the membrane which is the lysis tension at around $3.6 \%$ of its initial area. Therefore, the stretching device should precisely dilate the SLBs up to this and slightly above to induce elevated membrane tensions. The millifluidic device was developed by Laura Turco through replica modeling of the stretchable substrates for cells from Huh et al. that mimic the biological environment in an organ. ${ }^{60-61}$ The fabricated milli-fluidic device provided a thin PDMS sheet spanning between two side channels, where air pressure was reduced to achieve an anisotropically increased surface area. This anisotropy of surface dilatation was intended to receive different area dilatations in one sample that could include unstressed SLBs and SLBs with increased membrane tension in one sample. Spread SLBs on the surface of the thin PDMS sheet could be stretched with this device to induce and measure membrane area changes through the use of confocal microscope in conjunction with the thresholding technique of LI et al. ${ }^{62}$

In this chapter the results for the dilatation of the substrate with attached SLBs are shown to prove that it is possible to change the area of the SLBs by stretching the underlying PDMS substrate. In addition, membrane fusion was investigated through the use of SNARE proteins that were incorporated into the LUVs (synaptobrevin) and GUVs ( $\triangle$ N49-complex). Passivation of the PDMS surface, samples without SNAREs and SLBs on pure glass were measured to prove the validity of the used technique. FRAPmeasurements proved lipid mixing of LUVs with the SLBs.

The membrane tension for each membrane patch was calculated through the measured value of area change from the confocal images. LUV fusion efficiency on the SLBs was detected by a measure of fluorescence intensity increase of the LUV dye at the SLBs. Comparing the fusion efficiency with the membrane tension of each SLB yields the final result that elevated membrane tension increases membrane fusion efficiency. 


\subsubsection{Dilatation of SLBs on hydrophilic PDMS surface}

Supported lipid bilayers (SLBs) on hydrophilic surfaces can be produced by spreading of different kinds of vesicles. SUVs and LUVs normally form bilayers that fully cover the surface of the substrate if the appropriate vesicle concentration in the solution was set. A fully covered substrate surface with a lipid bilayer is a commonly used subject for the investigation incorporated trans-membrane proteins or peptides forming defined clusters, lipid segregation into different phases that can be investigated by fluorescent microscopy, infrared spectroscopy, ellipsometry and atomic force microscopy. ${ }^{30,}$, $110-113$

Here I used a polymeric substrate with a $180 \mu \mathrm{m}$ thin Polydimethylsiloxane (PDMS) sheet on a molded PDMS layer so that the thin sheet was stretched by applying a lower pressure to the side channels of the spanned sheet in between. The surface of the thin PDMS sheet in the middle was thereby stretched to a defined area dilatation of the surface because the distance increase of clusters of fluorescent beads on the surface and incorporated into the thin sheet were measured. At the next step a fully covered PDMS surface with a lipid bilayer was used as a first insight to applied membrane tension on a polymeric and dilatable surface. Figure 5.18 represents the dilatation of a membrane covered PDMS surface which was measured by the master student Jörn Dietz. Green fluorescent beads were incorporated into the thin PDMS sheet also represented in figure 4.5 in chapter 4.2.2 so that the dilatation of the PDMS sheet is visualized as a reference to the membrane on the surface. Producing a lower pressure in the side channels of the PDMS layer, the SLB on the PDMS sheet is dilated in the same direction with the oxidized PDMS surface because of a strong adhesion of the lipids to the polymeric support. This was also found and described in a paper by STAYKOVA et al. where they stretched and compressed their membranes also on a PDMS surface. ${ }^{58} \mathrm{It}$ was possible for me to reproduce their results in a first step to my goal of measuring the membrane tension of defined membrane patches by a direct access to the occupied membrane area on the dilated PDMS sheet. At first a reference state was measured with fluorescence microscopy to ensure that the initial surface coverage is complete and no defects in the SLB occur. As it can be seen in figure $5.18 \mathrm{~A}$ the surface is completely covered with the red membrane without any deformations or cracks in it. Figure $5.18 \mathrm{~B}$ represents a first suction of the syringe pump that induced the stretching of the PDMS surface that gained a surface dilatation $\epsilon_{\mathrm{x}}=4.1 \%$ which is slightly above the maximal area increase for this lipid composition of $3.6 \%$ which was stated as the rupture point in chapter 2. For that reason it was possible that the small cracks on the membrane could be seen as a first hint of a strongly tensed membrane that starts to rupture. The image in figure $5.18 \mathrm{C}$ is a very nice proof of a ruptured membrane on the stretched PDMS-support. Vertical cracks occurred all over the image in a parallel way to each other. At that stage, the surface dilatation was around $7.6 \%$ which is much higher than the predicted area increase at the lysis tension. Releasing the surface dilatation by increasing the air pressure in the side channels, yields a relaxed membrane. In the left part of the picture in figure $5.18 \mathrm{D}$ small membrane tubes can be seen that indicate that the SLB area is compressed so that the smallest possible area per lipid is reached. This could be explained by a slightly compressed surface and by the spreading of initially adhered LUVS on the SLB that were incorporated into the SLB while the dilatation was executed. This documentation of dilating SLBs on the fabricated PDMS surface of the membrane stretcher device shows that it is possible to dilate membranes between $0-7.6 \%$ of their initial area which can be measured by fluorescence microscopy. 

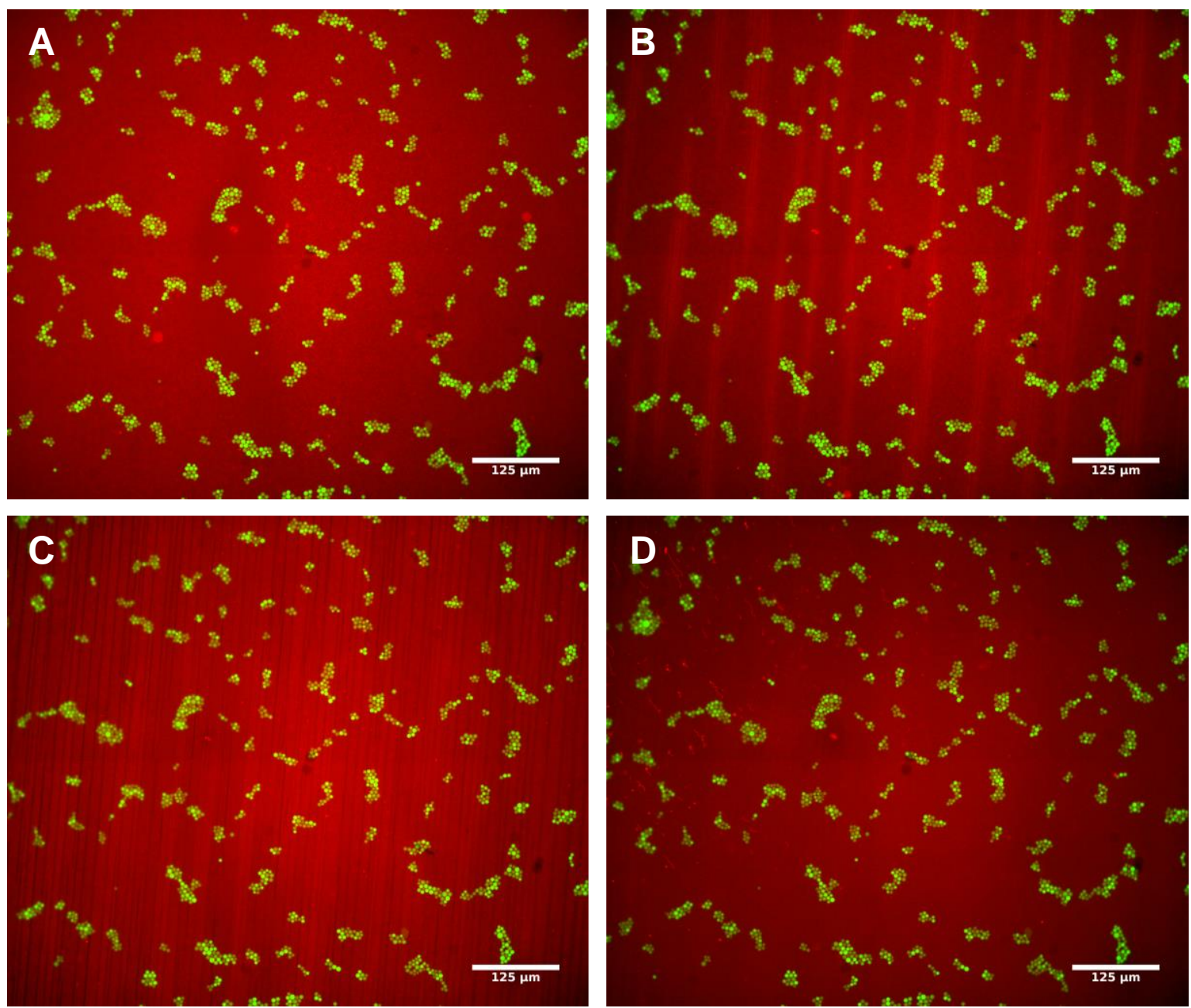

Figure 5.18. Dilatation of PDMS surface fully covered with a lipid bilayer. A) A SLB (red) covered the whole PDMS surface. Green fluorescent beads were incorporated into the thin PDMS sheet below the surface to measure the dilatation. B) The dilatation between the beads at the left and right was around $4 \%$ and the SLB on the surface started to rupture. C) Vertical cracks in the SLB can be seen because of the high area dilatation of around 7.6\%. D) Relaxing of the PDMS surface led to the compression of the SLB and a few membrane tubes formed at the left side of the image.

Cracks and defects in the SLB occurred at around 4\% area increase and could be clearly identified in the fluorescent images. In summary, dilatation of the PDMS surface led to an increase of membrane area that showed cracks at higher dilatation whereby compression of the SLB induced the reformation of a fully covered lipid bilayer with no holes but some membrane tubes. 


\subsubsection{Defined membrane patches}

In the next step defined membrane areas on the PDMS substrate were obtained by spreading of GUVs so that a dilatation of the support could lead to an area increase of the membrane patches. Previously, it has been shown by STUBBINGTON et al. that membrane patches on differently oxidized PDMS surfaces can be stretched. In comparison with STUBBINGTON et al. our PDMS surfaces were oxidized with oxygen plasma for 10 seconds which is in between their two oxidation processes of 3 and $30 \mathrm{~s} .{ }^{59}$

Interestingly, it was possible to reproduce the results of STUBBINGTON et al.. Membrane sliding, rupture and area increase due to surface dilatation occurred in the same sample. Membrane sliding means that the area of the membrane patch stays nearly constant while stretching the underlying PDMS support. Membrane holes appear on some strongly adhered membrane patches after stretching of the substrate.

To obtain defined membrane patches on a hydrophilic surface, GUVs incubated on the hydrophilic surface which leads to a strong adhesion site. Introducing a hyperosmotic buffer induces spreading of the GUVs on the surface to defined membrane patches. The amount of GUVs on the surface was important for the spreading of define membrane patches. A large number of adhered GUVs in near vicinity to each other would lead to large membrane patches that could not be measured as independent membrane patches. Therefore, it was necessary to add a small amount of GUVs to substrate. At low GUV-concentrations only one membrane patch could be measured within on sample because of the limited scanning region of the confocal microscope. For example, a large membrane patch of $6229 \mu \mathrm{m}^{2}$ is shown in figure 4.6 (chapter 4.2.2) that has the advantage of visualizing the area increase due to the dilatation of the substrate surface. The two threshold regions before and after surface dilatation can be clearly identified and compared to each other. From this comparison, it can be seen that an area change of $(1.4 \pm 0.1) \%$ is clearly detectable along the $x$-axis at which the surface was dilated. For the measurement of membrane area the LI-thresholding technique described in chapter 4.2.2 was the tool of choice to threshold the images and to measure the change in area of the threshold ROI of each membrane patch. 


\subsubsection{Passivation and control sample}

To determine whether fusion of LUVs to the membrane patches on the surface is mediated by SNAREs and an applied membrane tension through surface dilatation the hydrophilic PDMS surface was passivated and a control measurement was done. LUV fusion to SLBs and spreading of the LUVs on the PDMS substrate was tested in a sample without any further passivation of the substrate surface around the membrane patches and without the use of SNARE proteins. In the following figure 5.19, two images are shown representing the two fluorescently labeled lipids: A594 in the SLBs (A) and A488 (green) in the LUVs (B). The images of the SLBs and LUVs in figure 5.19 were measured by Jörn Dietz. The right image represents the channel of the green LUV dye and obviously shows that LUVs all over the pictures occur. All membrane patches show green fluorescence with different intensities whereas the PDMS surface is sprinkled with a lot of green intensity maxima indicative for LUVs. This blind sample documents that it is necessary to implement a passivation technique to prevent undefined LUV fusion at the edges of the SLBs and spreading of the LUVs to the hydrophilic PDMS surface. Not only was the passivation of the surface important but also preventing cracks and deformations of the PDMS surface.
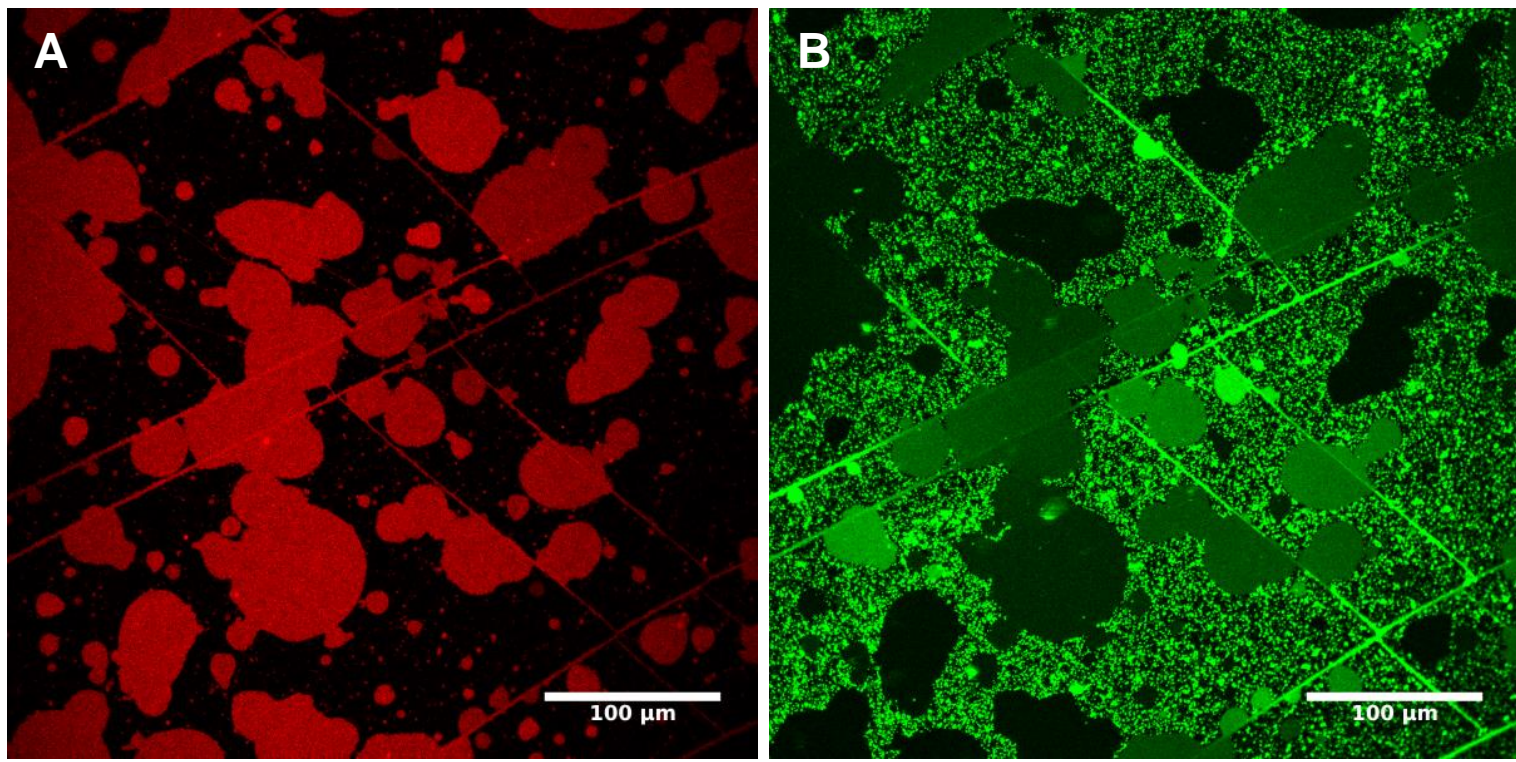

Figure 5.19. A) Spread GUVs on the PDMS surface that from SLBs (red). B) Addition of LUVs (green) even without SNAREs led to LUV fusion on SLBs because of the spreading on the hydrophilic surface. Cracks in the PDMS surface also promote LUV fusion to the SLBs originating from spread GUVs.

As a consequence, the protein BSA was introduced to passivate the hydrophilic PDMS surface around the SLBs to obviate all the mentioned occasions of undefined LUV fusion events. The passivation method was performed as described in chapter 4.2.1. As a proof for the passivation of the substrate surface a control sample was set up to see whether there is LUV fusion present without any SNAREs on a passivated surface. In figure 5.20 A two membrane patches (blue) are shown after the incubation of LUVs (red) without any SNARE proteins in the sample. Additionally the PDMS surface was dilated by an increase of the syringe volume $(2 \mathrm{~mL})$ connected to the side channels of the substrate. No LUV fusion on membrane patches could be detected. The fluorescence intensity area scan drawn in figure $5.20 \mathrm{~A}$ 


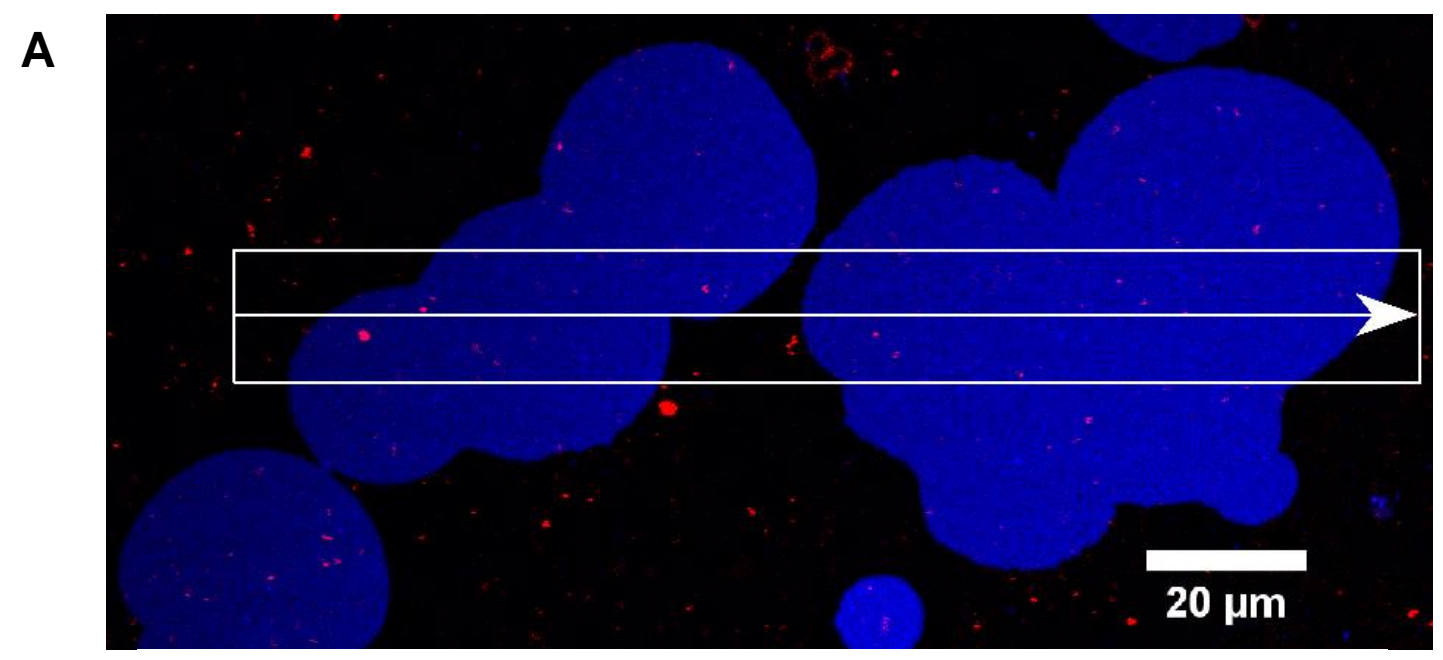

B

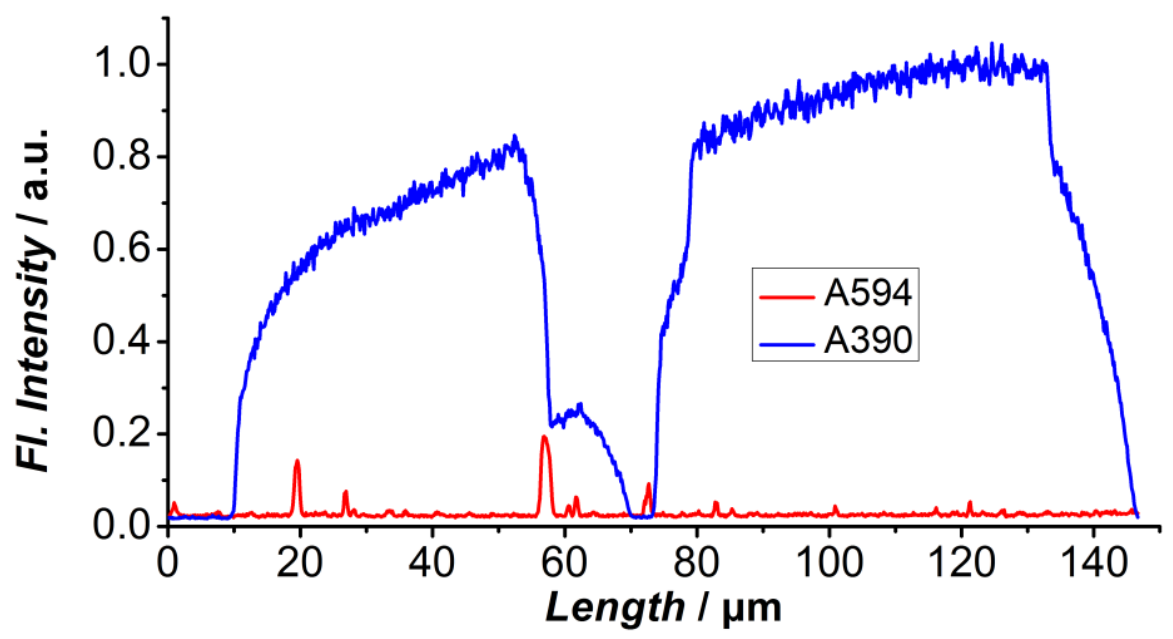

Figure 5.20. Blind sample shows no LUV fusion to the SLBs after passivation of the PDMS surface around the membrane patches. A) The two channel image (blue SLBs: A390; red LUVs: A594) shows only a few LUVs on the SLBs and on the surface after passivation. The fluorescence image was measured after stretching and relaxation of the PDMS sheet and incubation of LUVs without any SNAREs. The white box depicts the area scan. B) The area scan of (A) displays the average fluorescence intensity of both dyes. LUV fusion on the SLBs was absent because there was no countable fluorescence intensity increase of the LUV dye A594 on the SLBs.

is represented in the graph (B) for the SLB dye A390 and LUV dye A594. Out of that control sample, it can be concluded that the passivation of the hydrophilic PDMS surface around the membrane patches with BSA was successful to prevent undefined fusion events. After this preliminary work was finished then the investigation of membrane fusion as a function of lateral membrane tension induced by the polymer support of the target membranes could be performed. 


\subsubsection{SLBs on glass surface}

The determination of membrane area change by the thresholding technique described in chapter 4.2.2 was very important for the calculation of membrane tension of each SLBs. A very important question about that technique is: How big is the error of that method, because the induced area changes were should be in the range of one to four percent of the initial area. To determine the deviation of membrane area, a glass slide was used as a substrate for the spreading of GUVs that form defined membrane patches. By moving the substrate with the SLBs on it to a slightly different position, readjusting the focal plane at the microscope and thresholding the scanned membrane patches the area deviation of the membrane patches can be achieved. This glass substrate served as a reference for the measurement of the maximal error for each membrane patch area. Although the glass surface is a different substrate compared to the PDMS membrane stretching device, the error for the thresholding technique should be the same on both substrates. In the following figure 5.21 A five membrane patches were measured after moving the positon of the membrane patches in the scanned region. The measured membrane area change is displayed in figure $5.21 \mathrm{~B}$. The error for the measurement of membrane area for each patch was in the range of $0.07 \%$ to $0.65 \%$ with an average of all changes of $\Delta\left|\Delta A / A_{0}\right|=(0.38 \pm 0.19) \%$.

With this analysis of SLBs on a glass surface, the error of the thresholding technique is smaller than $0.7 \%$ percent area change so that an area change of SLBs on the PDMS stretching device above $0.7 \%$ should be representative of an area increase and an induced higher membrane tension.

Changing the microscope settings like the LASER intensity or the detector sensitivity resulted in much larger error for the membrane area change measurement up to $1-2 \%$ so that in all measurements with the same sample the microscope settings stayed constant.

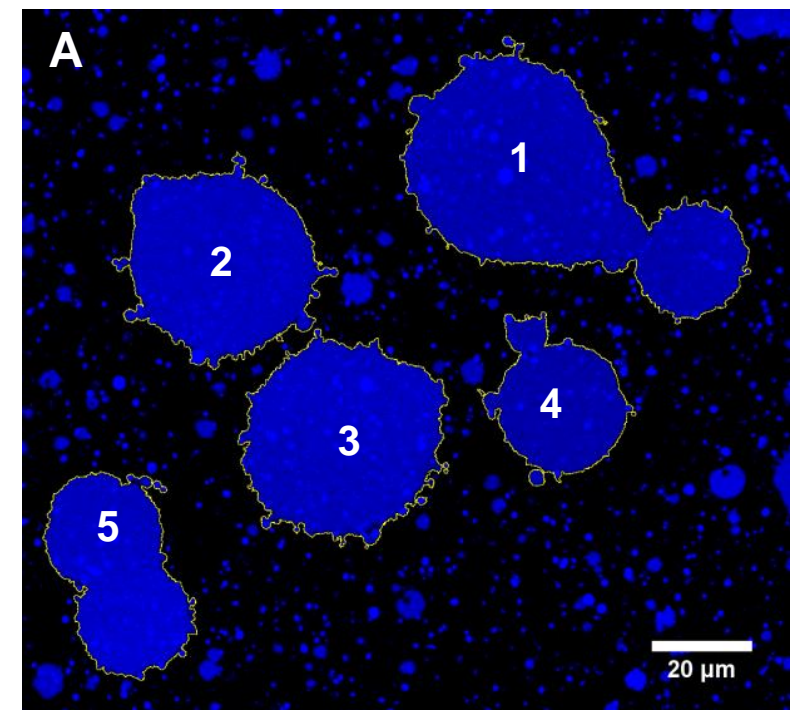

\section{B}

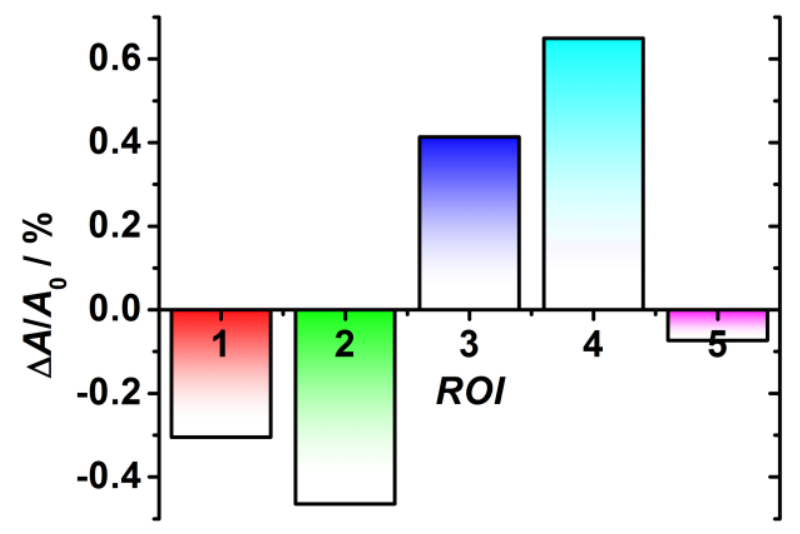

Figure 5.21. A) Threshold image of membrane patches on a glass surface for the error-measurement of membrane area determination. B) The measured area change is given in percent after changing the position of the membrane patches and readjusting the microscope settings. 


\subsubsection{FRAP experiments}

Fluorescence recovery after photobleaching (FRAP) was carried out to confirm LUV fusion on SLBs using SNAREs. Therefore, the dye A594 added to the LUVs was bleached with full LASER intensity for a few seconds and images were taken to record the recovery of the lipid dye into the bleached spot. In figure 5.22 a SLB (blue) after LUV incubation was bleached with the $561 \mathrm{~nm}$ LASER so that the LUV dye at the red ROI in figure $5.22 \mathrm{~B}$ shows a loss in fluorescence intensity from the dye A594. The fluorescence intensity for A594 showed a fast and nearly full recovery after $12 \mathrm{~s}$ (Figure $5.22 \mathrm{D}$ ) on the SLBs with only a small immobile fraction (15\%). A reference ROI (green) is shown in the figure 5.22 where the reference intensity was measured to compare the bleached $\mathrm{ROI}$ with a random $\mathrm{ROI}$ on the SLB.
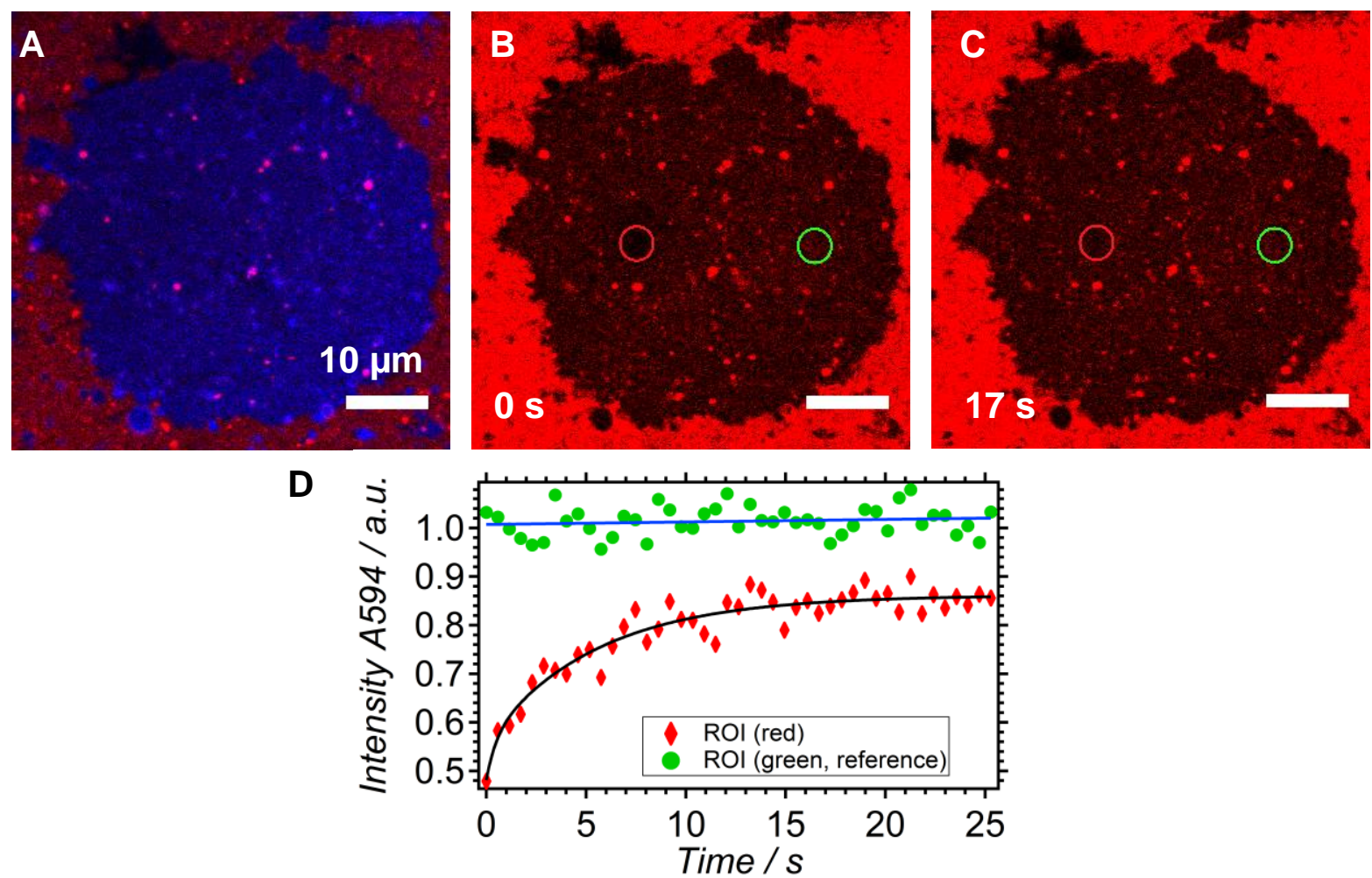

Figure 5.22. A FRAP measurement performed on a membrane patch (SLB) after fusion with LUVs by bleaching the LUV dye A594. A) The membrane patch (blue) displayed after the incubation with LUVs. B) Bleaching of the dye A594 with full LASER power was done at the position of the red circle. The enclosed area by the green circle was used as a reference. C) After 17 seconds the recovery of the LUV dye A594 back into the bleached spot was complete. D) The graph shows the intensity recovery as a function of time. An intensity of $85 \%$ of its initial value was eventually reached corresponding to an immobile fraction of $15 \%$.

Table 5.2 represents the measured half-times $\tau_{1 / 2}$ and bleaching spot radii $r$ that were measured in the four different FRAP experiments on SLBs with fused LUVs to calculate the diffusion constant $D$ for each SLB. Diffusion constants of the lipid dye A549 were calculated with equation (14) and are found to be around $0.9 \mu \mathrm{m}^{2} / \mathrm{s}$ with an immobile fraction of around $15 \%$. 
Table 5.2. Calculation of diffusion constants from four different FRAP experiments on SLBs by bleaching the LUV dye A594.

\begin{tabular}{|c|c|c|}
\hline$r / \mu \mathrm{m}$ & $t_{1 / 2} / \mathrm{s}$ & $D / \mu \mathrm{m}^{2} \mathrm{~s}^{-1}$ \\
\hline 2 & 1.2 & 0.8 \\
\hline 2.8 & 2 & 0.9 \\
\hline 2.7 & 1.7 & 1.0 \\
\hline 2.7 & 1.7 & 1.0 \\
\hline & average & $0.9 \pm 0.1$ \\
\hline
\end{tabular}

Fluorescence recovery after photobleaching was used to determine the mobility of lipids after fusion of LUVs with the SLBs. This mobility of the LUV dye A594 was proven with the experiment shown in figure 5.23. At the borders of the SLB in figure 5.23 it can be seen that many LUVs adhere to the PDMS substrate and maybe interfere with the SLB. To prove whether there is LUV fusion or lipid exchange at the borders of the SLB a ROI was bleached. In figure 5.23 B and C the bleached ROI (red circle) shows only a recovery of the dye A594 on the SLB and not on the PDMS where the LUVs adhere on the surface. The LUVs were bleached out and they stay bleached. The average fluorescence intensity in the red $\mathrm{ROI}$ in figure $5.23 \mathrm{C}$ remained very low compared to the green reference ROI. With this experiment it is proven that the lipid bilayer does not fuse with LUVs at the borders and that the LUVs do not spread on the PDMS surface.
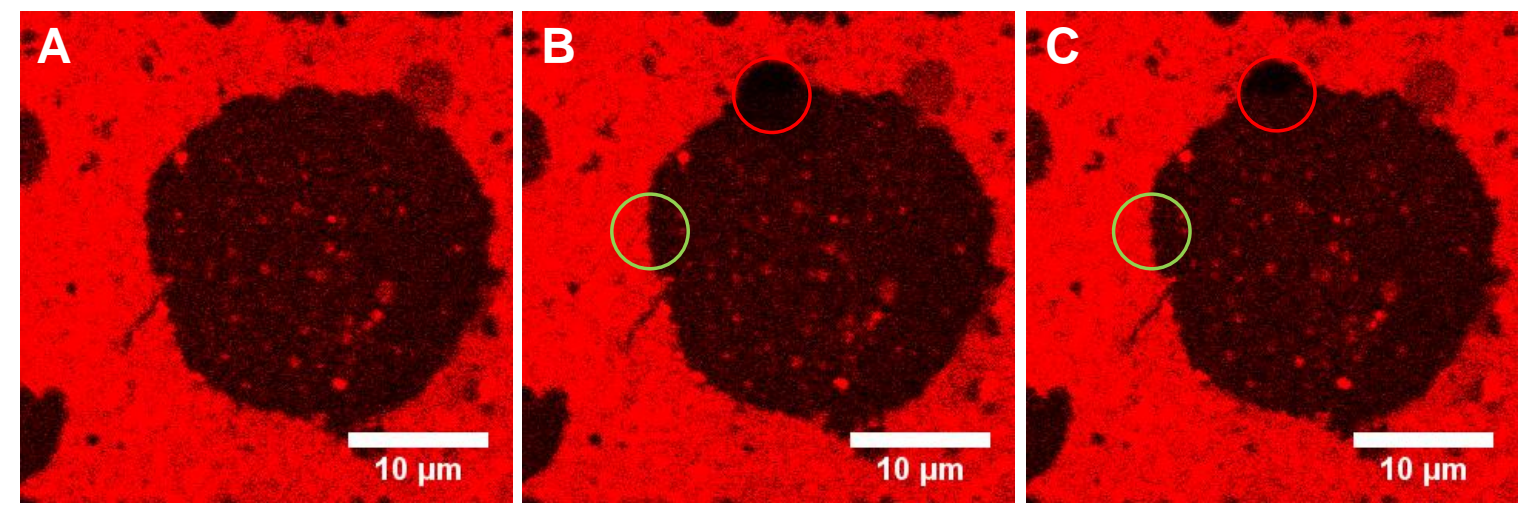

D)

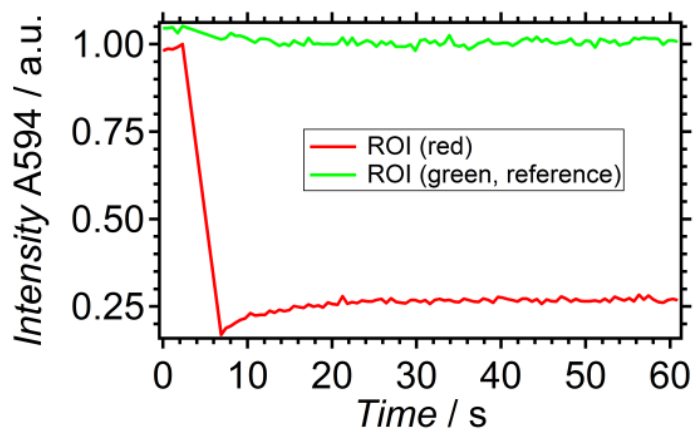

Figure 5.23. FRAP on the edge of a SLB patch. A)-C) Images taken during the FRAP measurement. The red circle shows the bleaching spot and the green circle serves as a reference. D) Fluorescence intensity of the bleached ROI (red) and reference ROI (green). 
Figure 5.24 shows the fluorescence recovery of bleached LUVs that are still attached to the SLB. The FRAP measurement confirms a shared mono- or even bilayer, i.e. incomplete vesicle fusion. The largest and therefore brightest vesicle in figure 5.24 A visible in the in the yellow circle only showed a no recovery after bleaching which might be indicative of docking (Figure $5.24 \mathrm{C}$ ). The recovery (figure 5.24 D) was fast, indicating that the dye was able to diffuse freely after (hemi)fusion between LUV and SLB. Therefore, it is assumed that the adhered vesicles formed a stable intermediate with an expanded fusion site that did not generate a large geometric barrier to lipid diffusion for the outer monolayer. ${ }^{29}$ FRAP experiments carried out on attached LUVs targeting to bleach A594 confirmed that lipids are shared between the SLB and the LUVs.
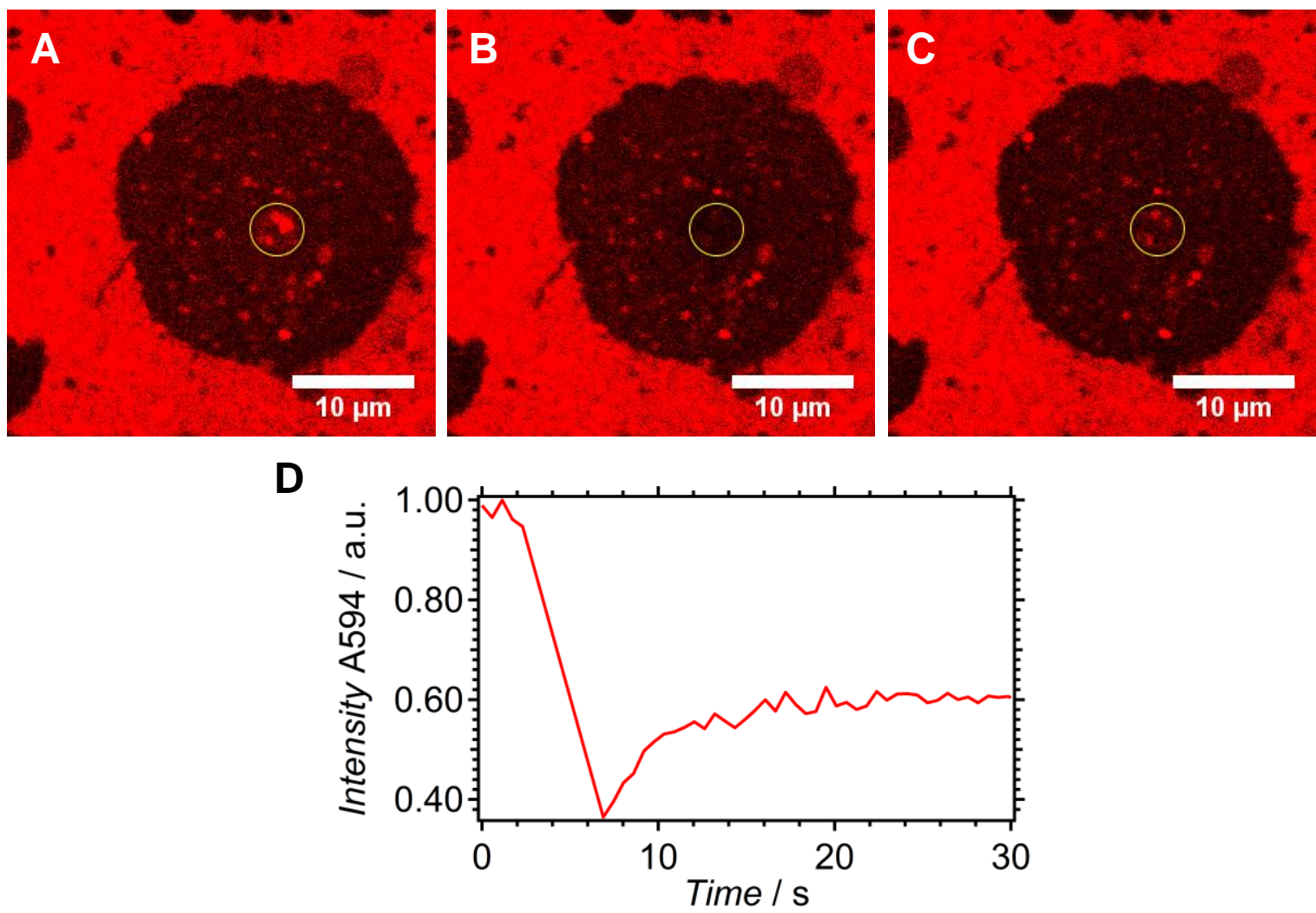

Figure 5.24. FRAP measurement of fused and docked vesicles. A) Vesicles in the ROI (yellow circle) are visible as bright red spot before starting the FRAP-experiment. B) Bleaching of the vesicles led to a complete loss of fluorescence intensity at the LUVs. C) After recovery two of the three vesicles showed recovery. The largest vesicle from image $(A)$, however, shows no recovery because this was only a docked or adhered vesicle. D) Mean fluorescence intensity collected from the entire circle during the time course of the experiment. 


\subsubsection{SLB area measurements on PDMS}

Five SLBs were chosen to demonstrate the method of measuring the SLB area before and after PDMS surface dilatation. The following example is representative for all measurements so that the membrane tension can be calculated through the measured area change with equation (6).

For the five SLBs depicted in figure 5.25, the membrane area of each SLB was measured a few times before and after dilatation and LUV incubation to induce membrane fusion. Interestingly, holes in the SLBs occurred after the dilatation of the PDMS surface which can be clearly seen in the fluorescent images. Not all membrane patches showed holes but these holes indicate that the lateral membrane tension of the SLBs was increased above the lysis tension so that the membrane ruptured on the PDMSsurface and generated holes. The area of the holes are subtracted by hand from the SLB area to yield the actual area of each patch if the thresholding technique was not able to detect the holes (figure $5.25 \mathrm{~B}$ ). The area of the SLBs ranges from $116 \mu \mathrm{m}^{2}$ for SLB 4 to $465 \mu \mathrm{m}^{2}$ for SLB 1 and compared to the example for the area change of the SLB with an area of $6157 \mu \mathrm{m}^{2}$ in figure 4.6 in chapter 4.2.2 the area of these SLBs is much smaller. Smaller SLBs could have led to higher error for the detection of membrane area change, because only a few pixel could make huge difference in membrane tension calculation. For example, a small error of only $1 \mu \mathrm{m}^{2}$ (around 60 pixels) in the scanned image could result in an area change of around 1\% for the smallest membrane patch in figure 5.25. Therefore, SLBs only with a size above $110 \mu \mathrm{m}^{2}$ were analyzed to reduce the impact of errors that originate from the accuracy of the microscope that is limited to the ABBE-Limit of optical resolution.
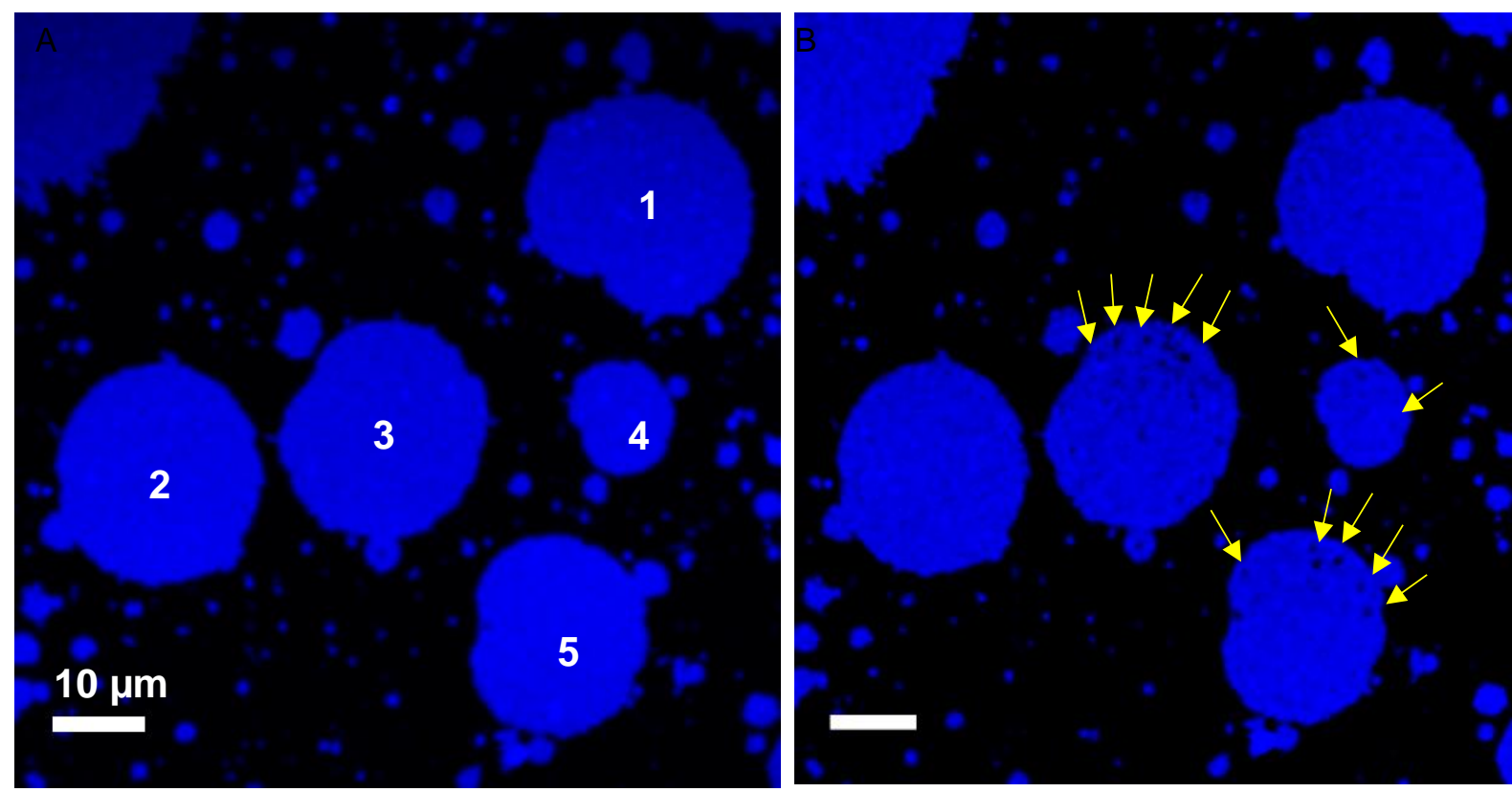

Figure 5.25. Comparison of SLBs before and after PDMS dilatation. A) Scanned image of five SLBs (blue) before surface dilatation reveal an even distribution of fluorescence intensity from the lipid dye A390. B) After stretching of the PDMS surface the SLBs 3, 4, 5 contain small holes as highlighted by arrows so that the measured membrane area stayed nearly constant to the initial one. 
A
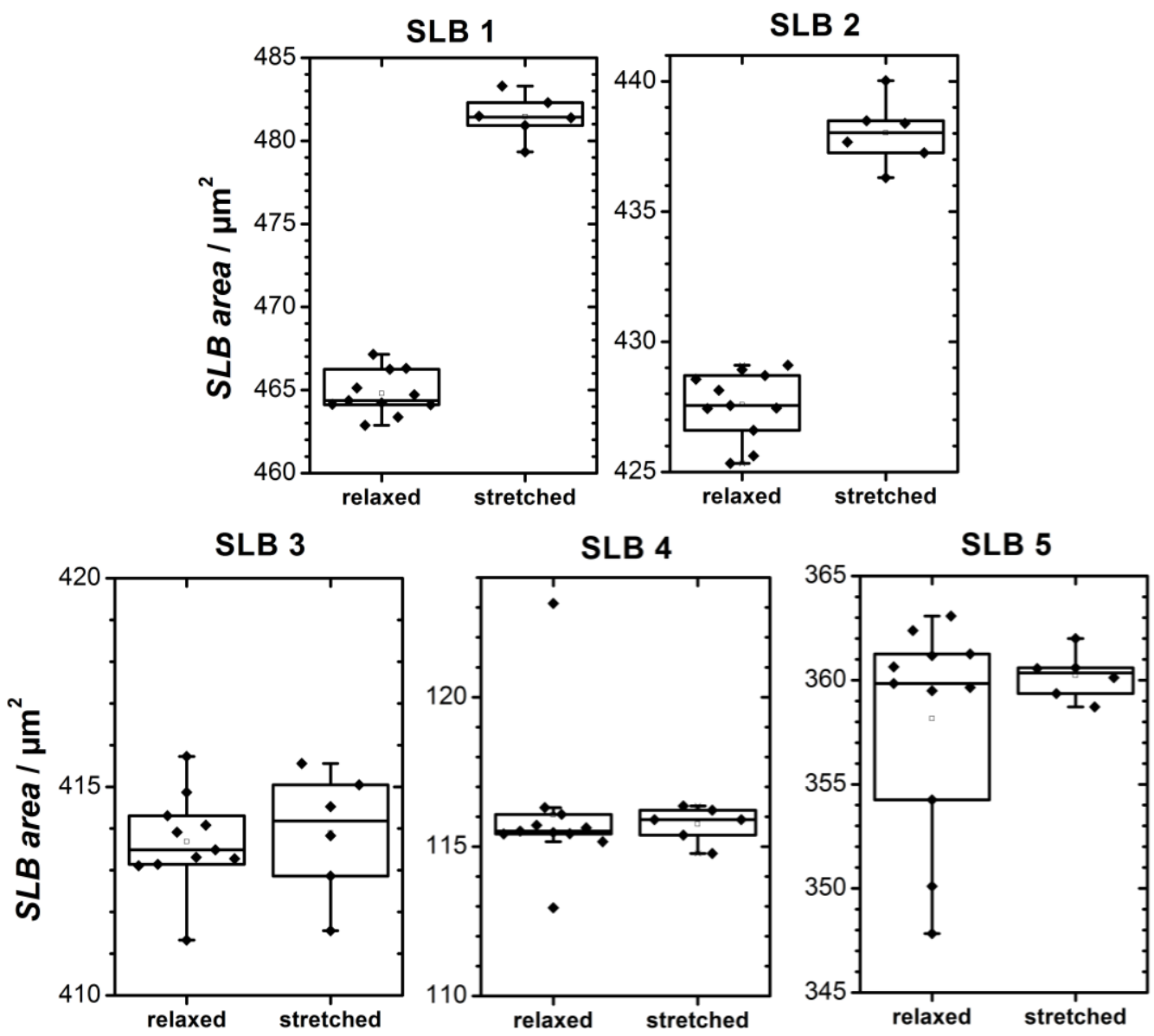

B

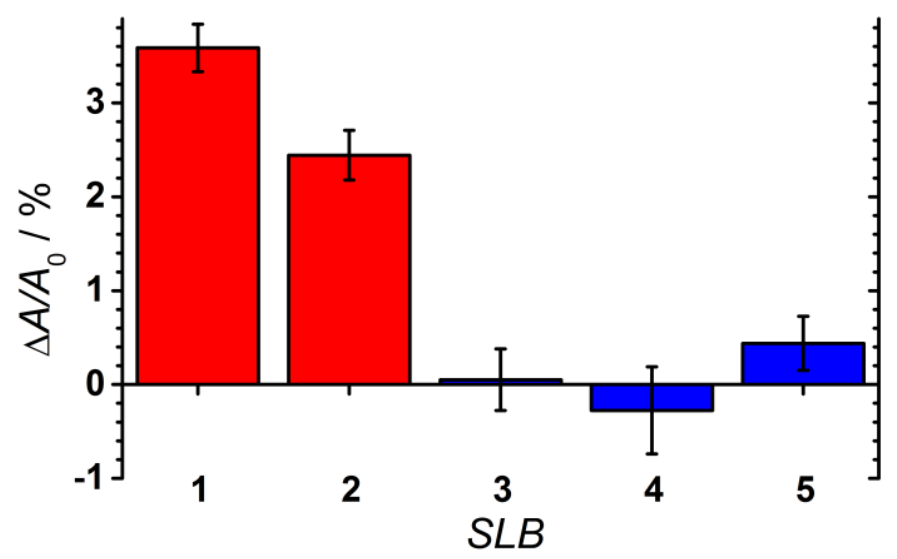

Figure 5.26. Measurement of membrane area change of the SLBs. A) The membrane area measurements with all data points for the SLBs $1-5$ from figure 5.25 , show an area change of the SLBs 1 and 2. (Box: 25\%, 75\%, whiskers 0-100\%). B) The graph plots the measured mean area change of the SLBs.

In figure 5.26 all measured data points are shown in graphs with boxplots to give an overview to the area change due to stretching of the PDMS surface. The measured area changes reveal that the membrane area of the SLBs 1 and 2 increase tremendously compared to the unchanged membrane area of the SLBs 3 to 5. Membrane holes appeared on the SLB 3 and SLB 5 with an area of $6 \mu \mathrm{m}^{2}$ and $5.5 \mu \mathrm{m}^{2}$, respectively. Very rarely did a membrane patch not change its area without showing detectable holes or cracks (SLB 4). This means that either the size of the holes was beyond the diffraction limit of 
the microscope or the membrane was not tightly enough attached to the PDMS surface so that membrane sliding occurred circumventing area dilatation of the SLB.

With these SLBs it was proven that the area of the membrane on the surface can be increased due to the surface dilatation despite some SLBs did not increased their area. This result of tensed and relaxed membrane patches in the same sample could serve as an intrinsic control sample for LUV fusion. With equation (6) described in chapter 2 the membrane tension for each membrane patch was calculated with an assumed area compressibility modulus of $0.28 \mathrm{~N} / \mathrm{m}$ that was determined by the compression of a GUV with an AFM-Cantilever. ${ }^{54}$ The following table 5.3 summarizes the measured average area increase and the calculated membrane tension. As a result, the whole span of possible membrane tension were measured with only five SLBs ranging from zero to $10 \mathrm{mN} / \mathrm{m}$ that is probably the lysis tension at which the membrane could rupture. However, SLB 1 at a membrane tension of $10 \mathrm{mN} / \mathrm{m}$ was stable and showed no holes or cracks whereas SLB 4 showed a slightly negative area change and the membrane tension is assumed to be zero.

Table. 5.3. Calculation of membrane tension with equation 6. The measured SLB areas led to a membrane tension range between zero and $10 \mathrm{mN} / \mathrm{m}$ which is the full range of possible membrane tensions from zero up to the lysis tension of the membranes.

\begin{tabular}{|c|c|c|}
\hline SLB No. & $\Delta A / A_{0} / \%$ & $\tau / \mathrm{mN} \mathrm{m}^{-1}$ \\
\hline 1 & $3.58 \pm 0.26$ & $10.0 \pm 0.7$ \\
\hline 2 & $2.44 \pm 0.27$ & $6.83 \pm 0.76$ \\
\hline 3 & $0.05 \pm 0.33$ & $0.14 \pm 0.92$ \\
\hline 4 & $-0.27 \pm 0.46$ & 0 \\
\hline 5 & $0.59 \pm 0.29$ & $1.65 \pm 0.81$ \\
\hline
\end{tabular}

Unfortunately, not all membrane tension regimes could be measured with only five SLBs. A few more membranes had to be analyzed for the investigation of membrane tension as a function of LUV fusion. Therefore, a total number of 30 membrane patches out of four measurements were used for the analysis of membrane tension and their fusion efficiency. 


\subsubsection{Fusion efficiency as a function of lateral membrane tension}

In this chapter, all measurements are represented with figures that depict the result of LUV fusion on SLBs supported on a PDMS surface. SLBs were dilated with on the PDMS stretcher device to induce an elevated membrane tension on the SLBs which was proven and exemplified in the chapter 5.2.1 and 4.2.2 before. The SLBs are shown in blue corresponding to their fluorescently labeled lipid A390 and the LUVs are shown in red corresponding to the fluorescently labeled lipid A594. A two channel image after the LUV incubation of 35 - 40 minutes was made with the CLSM so that it was possible to compare the fluorescence intensities on the SLBs to calculate the fusion efficiency with equation (15) from chapter 4.2 as a measure of fluorescence intensity increase of the dye A594 (LUV,A594) on the SLBS which was initially zero and increased due to LUV-fusion, docking and hemi-fusion. The fluorescence intensity of the dye A390 (ISLB,390), originating from the SLBs, served as an arbitrary reference (100\%). Importantly, LUVs are classified as docked if they did not shrink by incorporation of the lipids into the SLBs and at the same time do not display fluorescence for the blue dye A390. Hemi-fused vesicles do not shrink but show peak fluorescence from A390. SNAREs were used as membrane fusion promoters in all four measurements.

Figure 5.27 of measurement one shows the whole image of all SLBs containing the five ones from the chapter 5.2.6. In figure 5.27 the membrane patches of measurement 1 are displayed after PDMS dilatation and subsequent LUV incubation. Some membrane patches contain a brighter fluorescence intensity of the LUV dye compared to others. The mean fluorescence intensities of the LUV dye A594 differ between the membrane patches. Out of these images, 13 membrane patches could be used for the comparison of membrane tension and LUV fusion efficiency. Larger membrane patches at the left, right and above corner of the images were too big and ranged out of the scanning region that was limited to the microscope setup. Interestingly, from the image for the SLBs in figure 5.27 it can be seen that there are not many peak intensities that represent hemi-fusion of LUVs with a size above $300 \mathrm{~nm}$ in diameter, except for the three membrane patches SLB 8, 9, and 13 that contain a few hemi-fused LUVS. An explanation to that could be that most of the LUVs fused fully to the destined membranes.

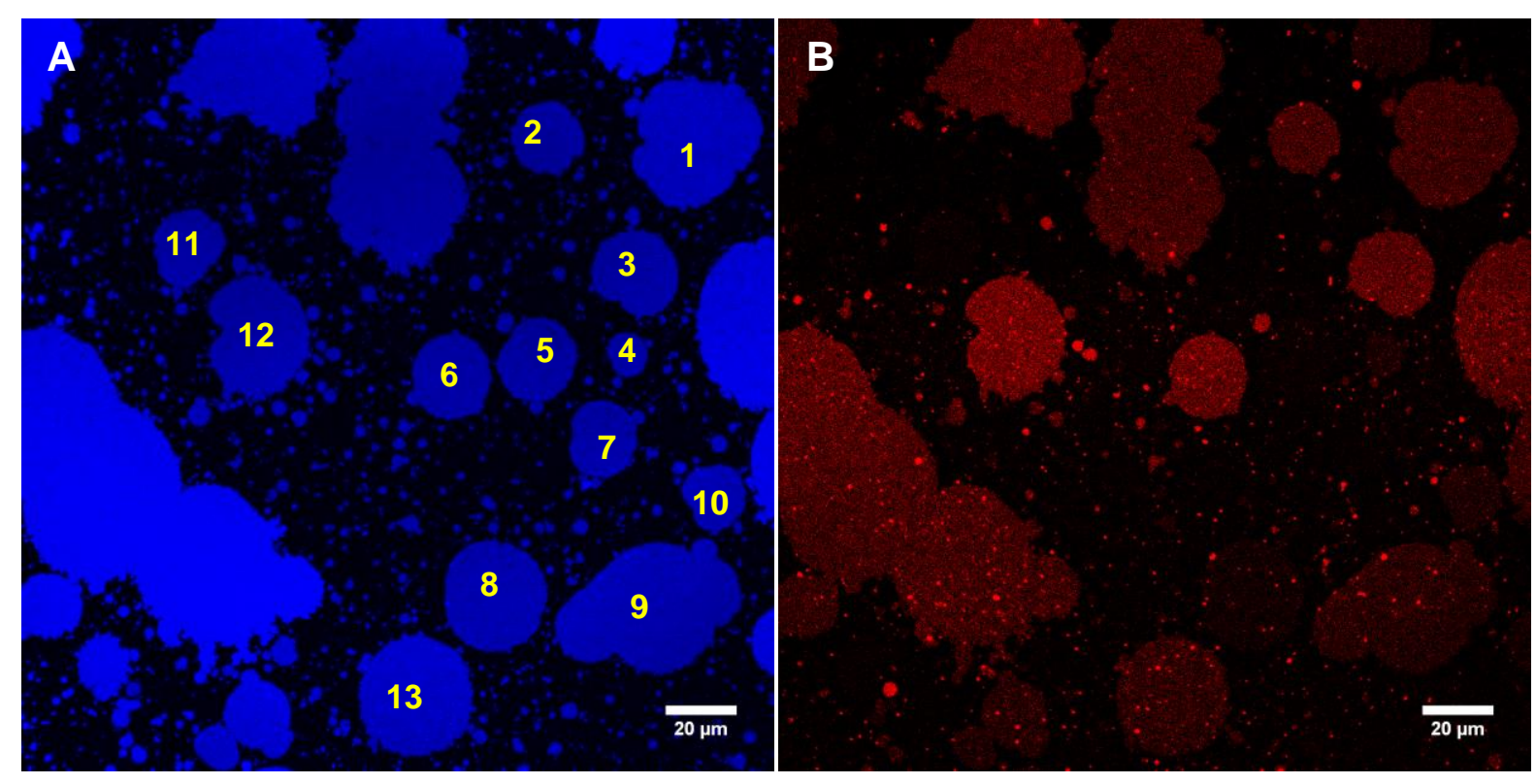

Figure 5.27. Measurement 1: Fluorescent images of blue SLBs (A) and red LUVs (B). 
A lack of LUV fusion can be seen on SLBs 4, 5, 7, 8, and 12. This is very important, because for these SLBs a lower membrane tension was calculated compared to the other SLBs that showed a higher fusion efficiency, for example the SLBs 1-3, 6, and 12.

Again, looking at the five membrane patches (SLBs 3-7 in figure 5.27) that were analyzed by their occupied area in chapter 5.2.6, they exhibit different fusion efficiencies of the LUVs. In figure 5.28 the two channel image of these five images is shown to illustrate the high fluorescence intensities of A594 on the SLBs 1 and 2 representing a higher fusion efficiency $F_{\text {eff }}$ (equation 15 in chapter 4.2 ) compared to the SLBs 3-5.
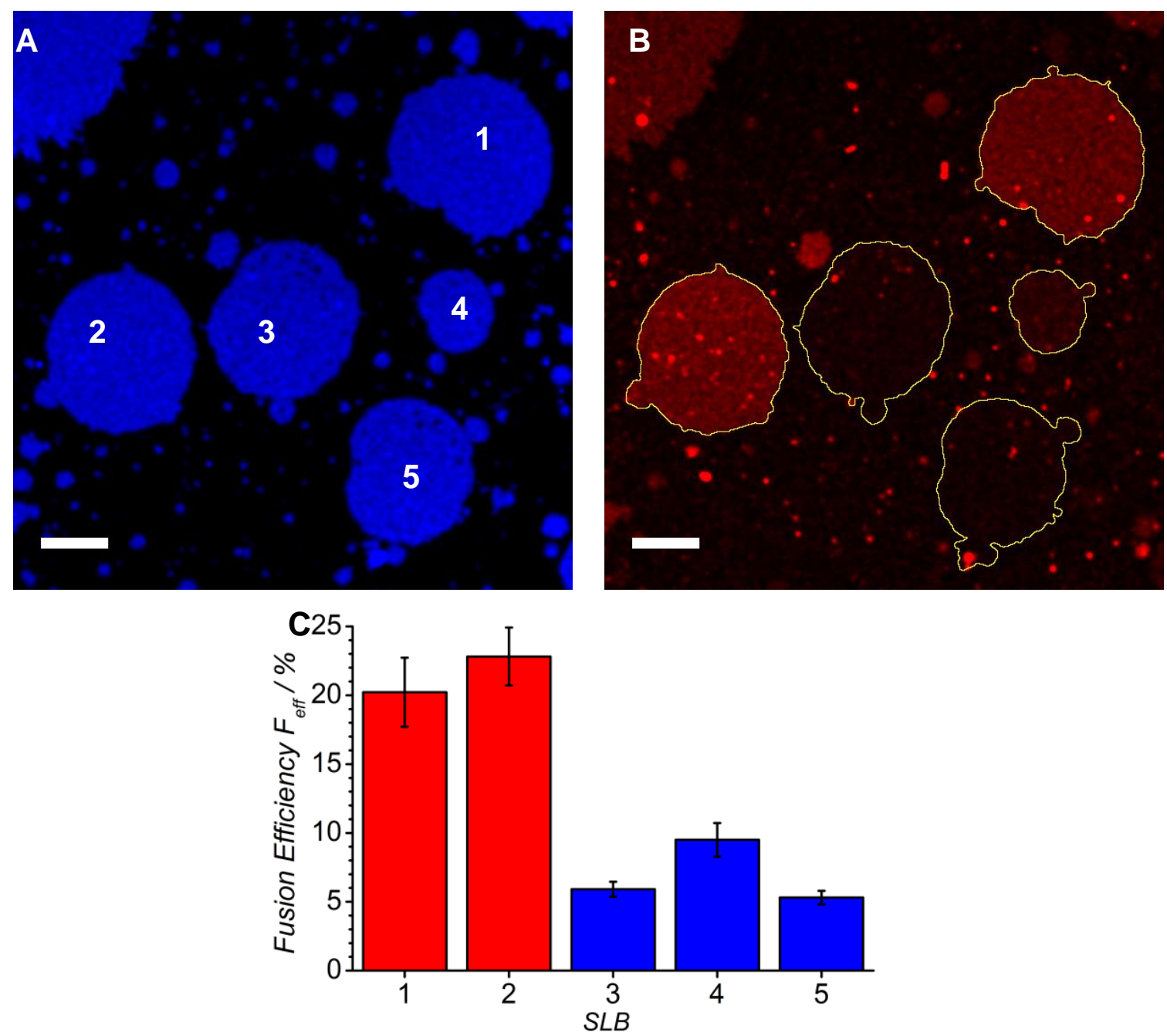

Figure 5.28. Measurement 1: Five SLBs (blue) and their fusion efficiency with LUVs (red). A) The SLBs 1-5 on the PDMS surface after surface dilatation. B) The same location but imaged with the LASER for the dye A594 reveals that SLBs 1 and 2 have an increased fluorescence intensity. Scale bar: $10 \mu \mathrm{m}$. C) The graph shows the fusion efficiency that was calculated with equation (15). The SLBs $3-5$ contain a very low fusion efficiency compared to SLBs 1 and 2.

This measurement reveals that the amount of LUV fusion is significantly increased at highly tensed membranes (SLBs 1,2) compared to SLBs (3-5) bearing nearly no membrane tension. As a consequence, all further measurements were analyzed in the same way to yield more data that proves this first insight into membrane fusion depending on lateral membrane tension. 
As a summary to measurement 1 , all fusion efficiencies were plotted into a graph as a function of membrane area increase for each SLB. Therefore, each point with the corresponding error bars represents one SLB from measurement 1. To conclude the results, drawn in the graph of figure 5.29, it can be stated that the area increase above $2.5 \%$ leads to a higher fusion efficiency of the LUVs on the dilated SLBs. An elevated membrane tension of two SLBs beyond the lysis tension, at an area increase of $3.6 \%(10 \mathrm{mN} / \mathrm{m})$, the fusion efficiency decreases. A further analysis of this decrease is given in a summary to all further results.

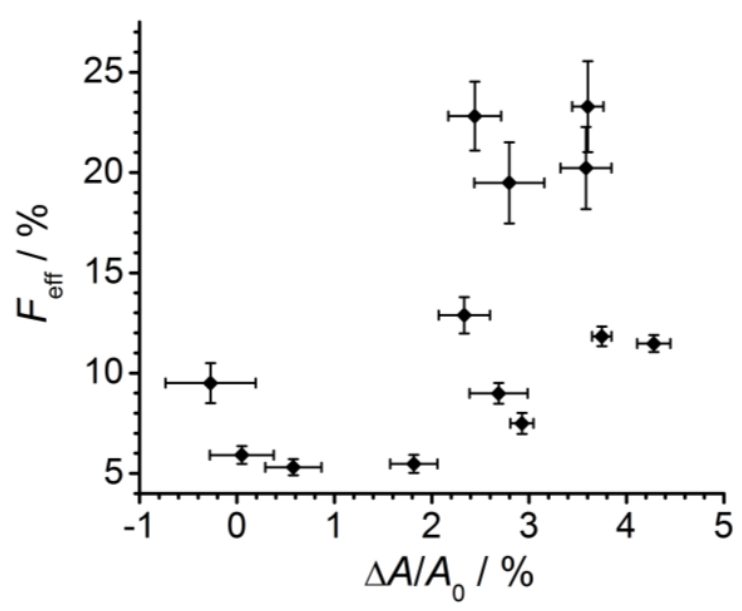

Figure 5.29. Measurement 1: All measured data points of fusion efficiencies as a function of membrane area change are drawn into the graph. The fusion efficiency increases at higher membrane areas that were induced by stretching of the substrate. Two SLBs above $3.6 \%$ area increase show a lowered fusion efficiency.

The measurement 2 is a good example of how the applied membrane area increase on the SLB influences LUV fusion. Two SLBs were imaged with the CLSM before stretching of the PDMS substrate (Figure $5.30 \mathrm{~A}$ ) and after the PDMS surface dilatation (Figure 5.30 B) at which the holes appeared at both SLBs with a size of $9 \mu \mathrm{m}$ for SLB 1 and $5 \mu \mathrm{m}$ for SLB 2, respectively. But after subtraction of the area of the holes, the measured area change of $(1.6 \pm 0.3) \%$ for SLB 1 was much greater than the area change for SLB 2 of $(0.37 \pm 0.25) \%$ (Figure $5.30 \mathrm{E}$ ). Figure $5.30 \mathrm{C}$ shows the blue channel for the SLBs where many small intensity peaks can be seen on the SLB 1 which means that many LUVs hemi-fused or fully fused but did not shrink with an incorporation into the SLB plane. Docking, hemi-fusion and fusion of LUVs occurs visibly more frequently on SLB 1 (figure $5.30 \mathrm{D}$ ). An explanation to this could be that the highly tensed membrane (SLB 1) with a calculated membrane tension of $(4.42 \pm 0.84) \mathrm{mN} / \mathrm{m}$ fused many large LUVs. The probability for that was higher on the tensed SLB compared to the SLB 2 at lower membrane tension. Comparing the membrane tension and the fusion efficiency with each other results in an elevated fusion efficiency parallel to the increased membrane tension as it was previously shown in measurement 1. Here, the measurement 2 with its two SLBs indicated also very clearly that LUVs can hemi-fuse or fully fuse on the SLB 1 without shrinkage. Many LUVs adhered to the hydrophilic and passivated surface so that the membrane area could not further increase at the side of each patch by vesicle fusion and incorporation of the vesicle lipids. The barrier, formed by LUVs adhered onto the PDMS surface, frustrates the area expansion of the SLB generated by fused LUVs. Therefore, relaxation 
of lateral tension in the membrane by injection of lipids from LUV fusion lasts only until the excess area, generated by stretching of the bilayer, is exhausted (Figure 5.30 SLB 1). After the lateral tension is fully relaxed by refilling the voids with external lipids from fusing with LUVs, arrested hemi-fusion and docking of intact LUVs remain the only option.

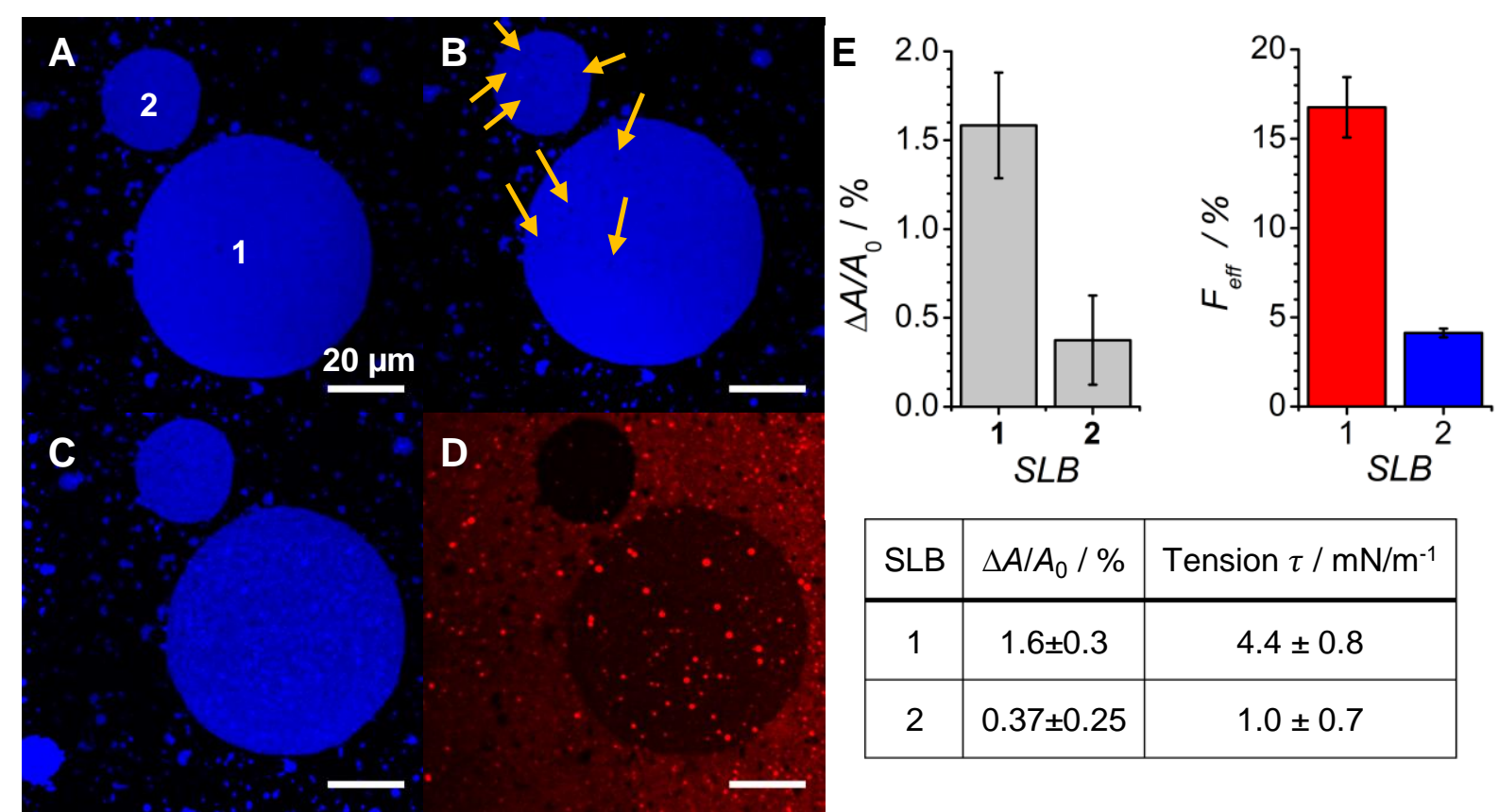

Figure 5.30. Measurement 2: A) Two membrane patches are shown in the relaxed state. B) Stretching of the PDMS substrate led to a strong increase in area for SLB 1 and only a small area change of SLB 2. Holes occur in both membranes. C) Many peak fluorescence intensites of the SLB dye A390 after LUV incubation are present on SLB 1. D) The fluorescence image for the LUV dye A594 is shown after the incubation of LUVs. The LUVs showed a higher affinity to the stretched SLB 1. E) The two graphs plot the measured area changes and fusion efficiencies. F) A table lists the calculated membrane tensions for the two SLBs.

In figure 5.31 A both channels were drawn in one image and LUVs, which fused to the SLBs, appear violet. Generally, lipid bilayer fusion between LUVs and the SLBs was detected at the points where peak fluorescence intensities overlapped for both channels. To prove hemi-fusion of LUVS on SLB 1, a fluorescence intensity profile area along one axis was measured where the intensities of the dyes were averaged at each point on the length scale to compare the fluorescence intensities of the lipid dyes A594 and A390 (Figure 5.31 B). Docked vesicles that do not emit blue light can only be found on SLB 2. The line scan through both membranes displayed in figure $5.31 \mathrm{~B}$ shows a higher mean fluorescence intensity at SLB 1 with peaks originating mainly from adsorbed LUVs. The area scan A1 in figure $5.31 \mathrm{~B}$ illustrates that the average fluorescence intensity of the LUV dye A594 at SLB 1 is higher compared to SLB 2. LUVs on SLB 1 produce small intensity peaks in the measured channel of A594. At some points on the length scale in the red channel and the blue channel showed small peaks that indicates some fusion of the LUVs to the SLB 1. Therefore, the line scan L2 (Figure $5.31 \mathrm{~B}$ ) displays two peaks, the first at $4.5 \mu \mathrm{m}$ length and the second at about $12 \mu \mathrm{m}$. The first peak at $4.5 \mu \mathrm{m}$ corresponds to a LUV that shares both monolayers with the SLB. Since full lipid insertion is no longer possible due to the inability of the SLB to expand laterally, the fused LUV stays arrested on top of the SLB but shares lipids with the 

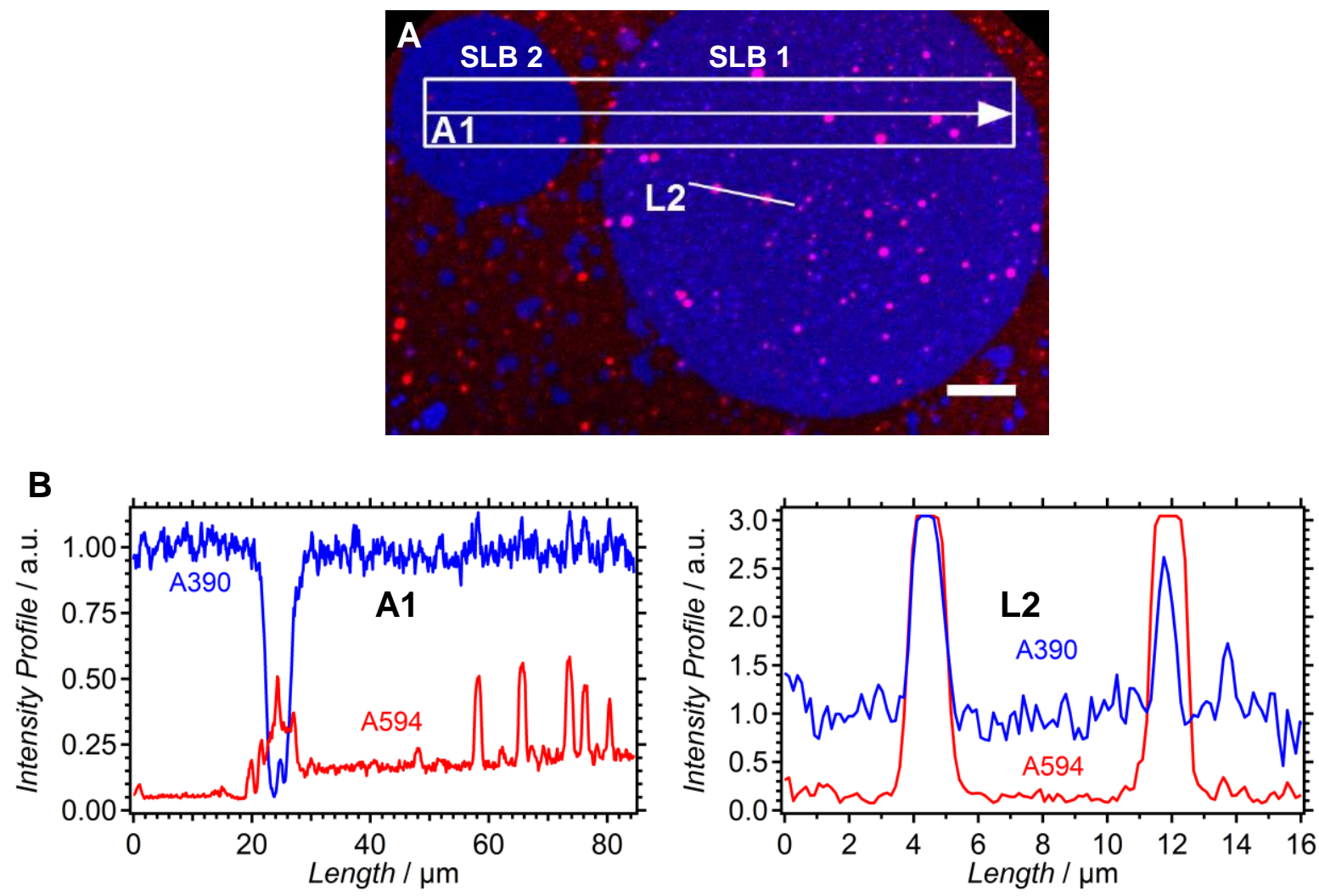

Figure 5.31. Fluorescence microscopy images of membrane patches illustrating docking, hemifusion and full fusion with LUVs. A) Fluorescence image (red and blue channel) showing two membrane patches after applying lateral stress and a white box at which the average intensities of dye A390 and A594 are measured and plotted in (B). B) The area scan (A1) shows the mean intensity of both dyes taken from the white box shown in (A). The lipid dye A594 initially only present in the LUVs emits less light from SLB 2 with a tension of $(1.0 \pm 0.7) \mathrm{mN} \mathrm{m}-1$ compared to SLB 1 that reached a membrane tension of $(4.4 \pm 0.8) \mathrm{mN} \mathrm{m}-1$. The line scan (L2) crosses two vesicles, one sharing lipids of both monolayers with the supported patch at $4.5 \mu \mathrm{m}$ and one which is hemi-fused with the bilayer patch at $12 \mu \mathrm{m}$.

SLB. Hemi-fused LUVs as seen in the graph (L2) at the length of about $12 \mu \mathrm{m}$ exhibit a lower intensity ISLB,A390 because only the outer lipid layer of the vesicle is shared with the SLB.

In figure 5.32 the measurement 3 is shown containing the largest SLB with a size of $6246 \mu \mathrm{m}^{2}$ after surface dilatation. This membrane patch was also used in chapter 4.2.2 to describe and visualize the dilatation of the membrane area due to the extension of the PDMS surface. For this membrane patch an area change of $(1.44 \pm 0.03) \%$ with a corresponding membrane tension of $(4.03 \pm 0.08) \mathrm{mN} \mathrm{m}^{-1}$ was found leading to a fusion efficiency of $(13 \pm 1) \%$. The calculated membrane tension was probably large enough to induce an elevated fusion efficiency of LUVs on the SLB but also an increased amount of docked and hemi-fused LUVs. 

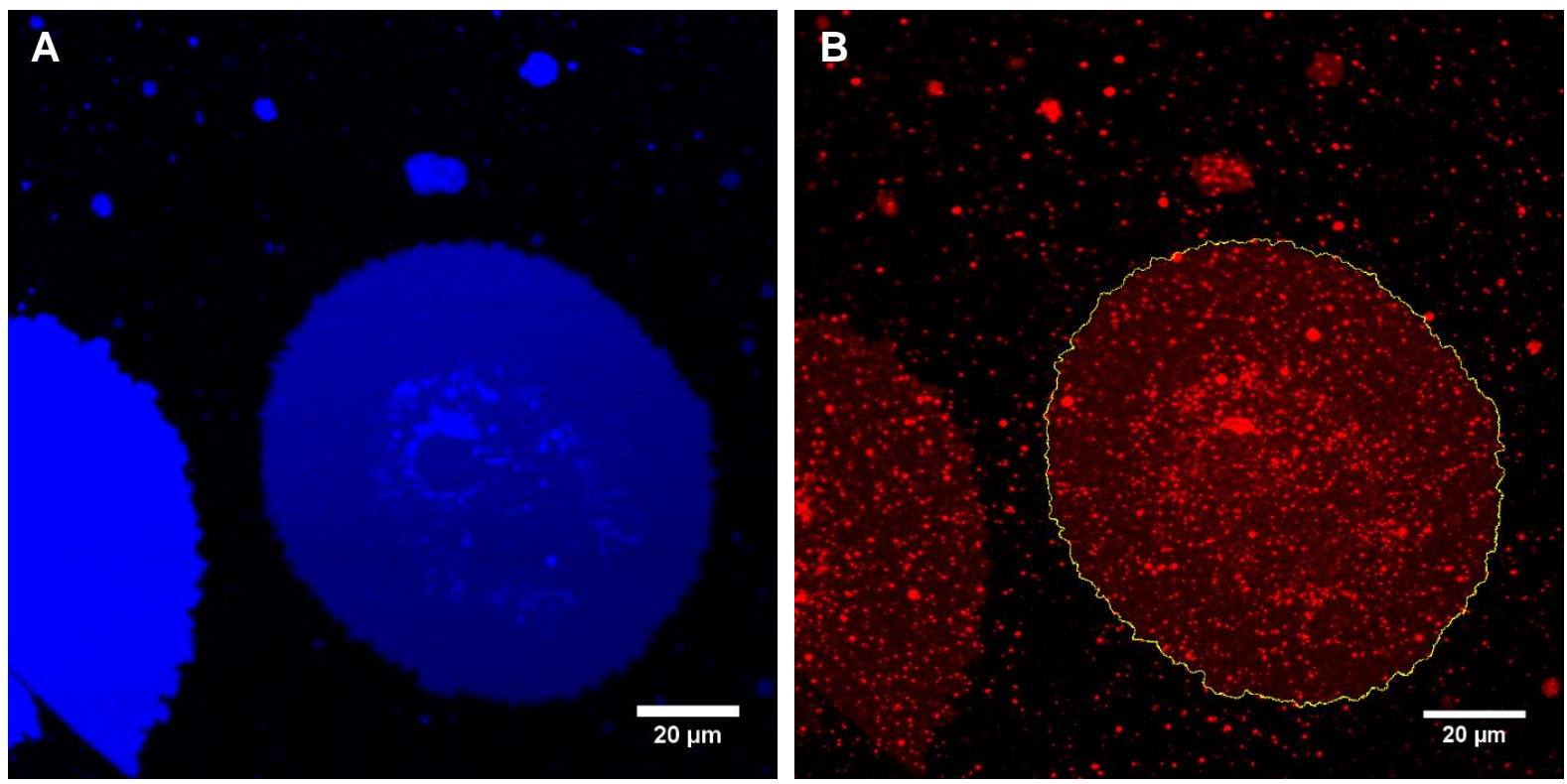

\begin{tabular}{|c|c|c|}
\hline$\Delta A / A_{0} / \%$ & $\tau / \mathrm{mN} / \mathrm{m}^{-1}$ & $F_{\text {eff }} / \%$ \\
\hline $1.44 \pm 0.03$ & $4.03 \pm 0.08$ & $13 \pm 1$ \\
\hline
\end{tabular}

Figure 5.32. Measurement 3: A) Fluorescent images of SLB (blue). B) Fluorescent image of LUVs (red) that fused, hemi-fused and docked to the SLB. The border of the SLB is drawn in yellow. C) The table lists the measured SLB area change and calculated membrane tension with the corresponding fusion efficiency.

In figure 5.33 A, another 14 SLBs are shown in the image and in figure $5.33 \mathrm{~B}$ the corresponding red channel for the LUVs is depicted. Many LUVs adhere to the PDMS surface because of nonspecific adsorption. Some membrane patches appear brighter in the red channel of dye A594 indicating LUV fusion. In measurement 4 many LUVs adhered to the hydrophilic PDMS surface but only the SLBs $1,2,5,6$, and 11 showed a significant fusion efficiency above 10\% corresponding to an area increase above $1.5 \%$, except SLB 11 showed only a very small area increase of only $(0.2 \pm 0.5) \%$ and a larger error.

The result of the measurement 4 is given in figure $5.33 \mathrm{C}$ where the fusion efficiency is plotted in a graph as a function of SLB area change. Every point in this graph stands for a single SLB. As a result the fusion efficiency increased with the dilatation of the SLBs. Area loss due to possible rupture events led to a lowered fusion efficiency compared to the dilated SLBs. Except SLB 11 revealed a higher fusion efficiency which could be a result of unspecific fusion but also the measured area change exhibited a large error which could be a result of a failed membrane area determination. Negative area changes occurred through the measurement 4 but did not showed a considerable fusion efficiency. Somehow the SLBs with a negative area change could have ruptured. Therefore, the membrane tension for the SLBs with a negative area change can be assumed to be zero or very small compared to the stretched SLBs, respectively. 

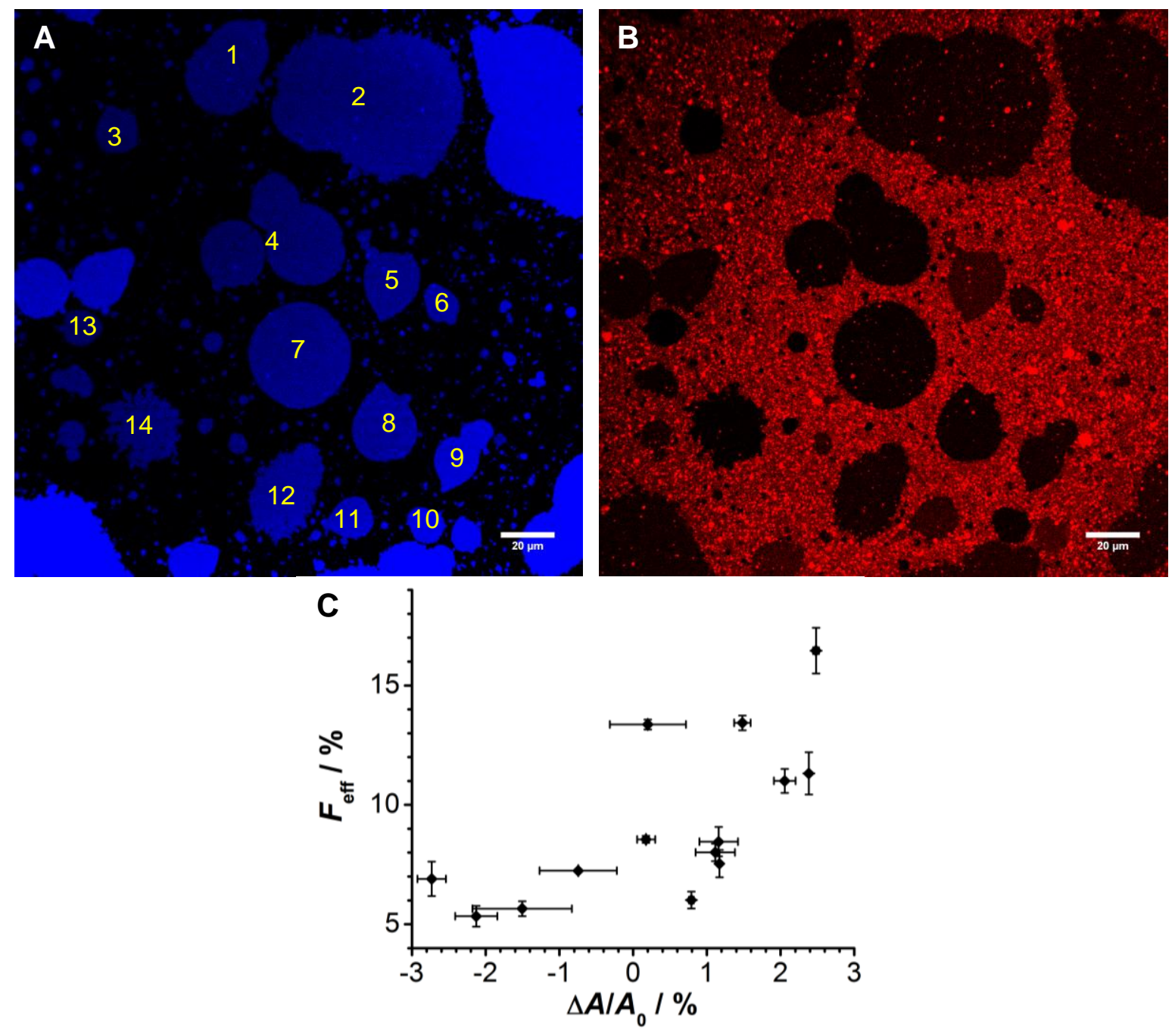

Figure 5.33. Measurement 4: Fluorescent images of SLBs (blue) and LUVs (red).

For all 30 membrane patches, the membrane area changes and relative fluorescence intensities were measured from two up to 10 images after LUV incubation and removing the LUVs from the buffer solution. The error between each measurement of the same patch was in the range of only a few $\mu \mathrm{m}^{2}$. Notably, smaller patches are more prone to erroneous determination of area change. Therefore, only membrane patches with a size range from $115 \mu \mathrm{m} 2$ to $6246 \mu \mathrm{m} 2$ were used for analysis of patch area and fusion efficiency ensuring that the overall area change error was below $1 \%$. The main sources of error was photobleaching of the oxidation-sensitive lipid dye A390 and small changes of the focal plane due to the dilated surface.

In summary, all 30 membrane patches from the measurements 1-4 have to be compared to obtain an overview to the dependency of LUV fusion according to induced area changes. Therefore, the graph in figure 5.34 plots the fusion efficiency versus the membrane area change $(A)$ and lateral membrane tension for all 30 membrane patches. The plot shows that the LUV fusion efficiency is raised through an increased membrane area that produced a higher lateral membrane tension. From the measured area changes the lateral membrane tension of each SLB was calculated with equation (6) so that the graph in figure $5.34 \mathrm{~A}$ can be translated to the fusion efficiency as a function of latera membrane tension with 
the assumption that in the used system the measured negative area changes were a result of ruptured membranes that exhibit zero membrane tension (figure $5.34 \mathrm{~B}$ ).
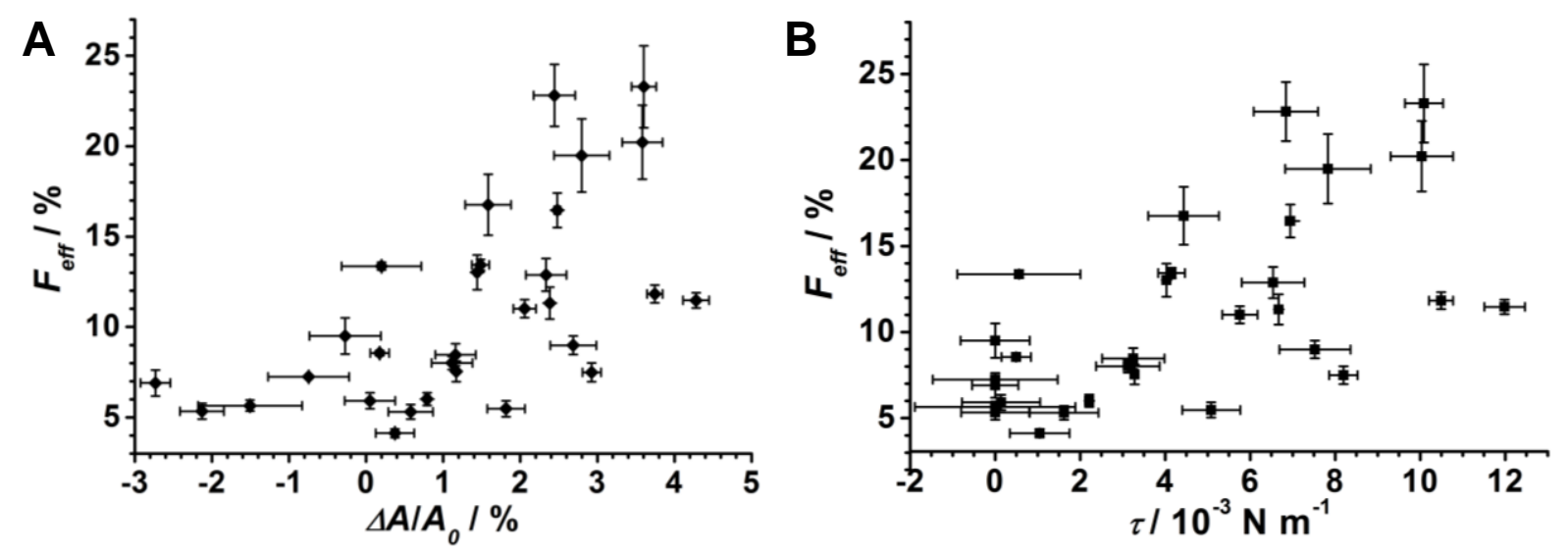

Figure 5.34. All 30 SLBs and their measured fusion efficiency as a function of membrane area change (A) or lateral membrane tension (B) where the negative area changes were assumed to exhibit zero membrane tension. The fusion efficiency was raised through an increase of generated lateral membrane tension up to $10 \mathrm{mN} / \mathrm{m}$.

For a compact summary of all measured data points $(N=129)$ a sectioned box plot in figure 5.35 is shown that comprises all measured points for all 30 SLBs. The lateral membrane tension here was divided into four different tension regions. The results show that membrane area changes from -3 to $1 \%$ with the corresponding membrane tension from zero up to $2.7 \mathrm{mN} \mathrm{m}^{-1}$ display an only moderate increase in relative fluorescence intensity /LUV,A594 / ISLB,A390 indicative of a fusion efficiency below (6.6 \pm 2.4$) \%$. Above a relative area increase of $1.2 \%$ (see also figure $5.34 \mathrm{~A}$ ), a significant increase in relative fluorescence intensity was measured. In the regime of $1-2 \%$ area dilatation corresponding to a membrane tension of $2.8 \mathrm{mN} \mathrm{m}^{-1}$ to $5.5 \mathrm{mN} \mathrm{m}^{-1}$, the relative fluorescence intensity increases up to $(11.7 \pm 4.5) \%$. A change from $1-2 \%$ to $2-3 \%$ of lipid bilayer area with a tension of $5.6 \mathrm{mN} \mathrm{m}^{-1}$ to $8.4 \mathrm{mN} \mathrm{m}^{-1}$ increased the fusion efficiency further to $(14.8 \pm 5.8) \%$. Further dilatation of the PDMS sheets yielded an area increases of the SLBs between 3-4.5\% with a corresponding lateral membrane tension of $8.5 \mathrm{~m} \mathrm{~N} \mathrm{~m}^{-1}$ to $12.5 \mathrm{mN} \mathrm{m}^{-1}$ of the bilayer but did not lead to further increase in fusion efficiency. Two SLBs with a measured area increase of $(3.75 \pm 0.10) \%$ and $(4.28 \pm 0.27) \%$ own a higher membrane tension than the typical lysis tension of a lipid bilayer which is $10 \mathrm{mN} \mathrm{m}^{-1}$. This could be a result of appearing holes or cracks in the SLB that were smaller than the diffraction limit of the used microscope. For that reason the detected membrane area and calculated membrane tension could be too large. The drop of fusion efficiency at very large membrane tension indicates that the optimal tension regime, that elevates the amount of fusion, lies in between $2.8 \mathrm{mN} / \mathrm{m}$ and $10 \mathrm{mN} / \mathrm{m}$.

In summary, it was found that the fusion efficiency increases considerably once a threshold membrane area dilatation of $1.2 \%$ corresponding to a membrane tension of $3.4 \mathrm{mN} / \mathrm{m}$ is reached. Higher tension values than $5.6-12 \mathrm{mN} / \mathrm{m}$ do not significantly enhance fusion. Therefore, it is proposed that the optimal tension range lies between $3.4-5.5 \mathrm{mN} / \mathrm{m}$ to considerably increase membrane fusion efficiency. 


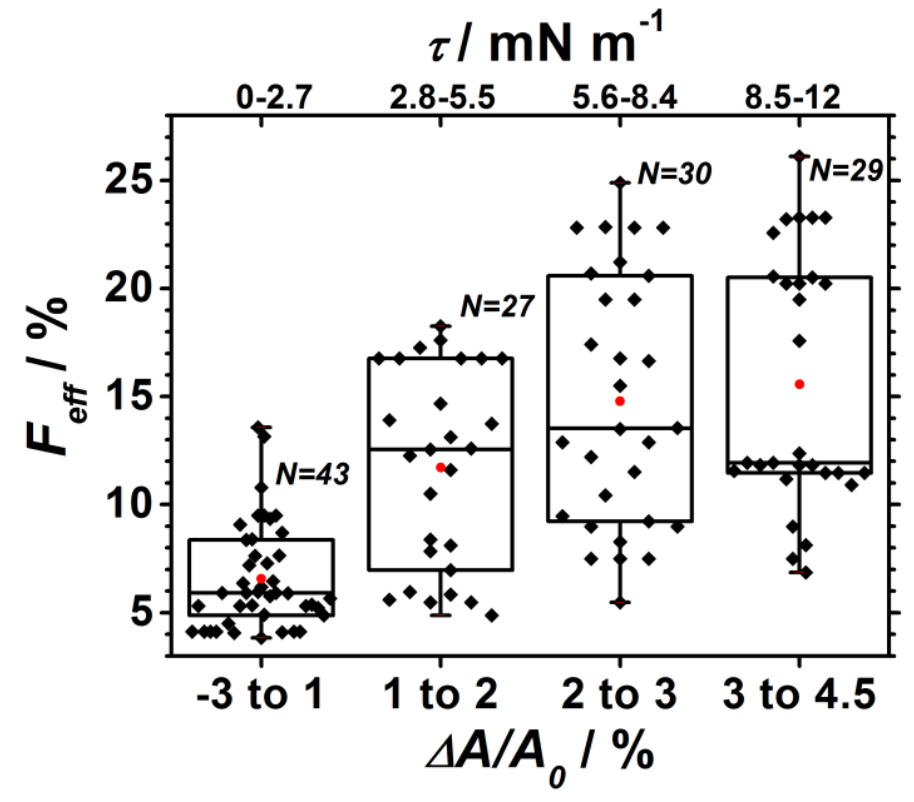

Figure 5.35. All data points $(N=129)$, each representative for one SLB measurement in one image from a total of 30 different SLBs, are plotted in the boxes of the graph. The boxes represent the 25 to 75 percentile. The median is shown as a line in the box and the red represents the mean value of all points. The fusion efficiency increases at a membrane tension of $2.8-5.5 \mathrm{mN} / \mathrm{m}$. A further increased membrane tension does not elevate fusion efficiency significantly. 


\section{Discussion}

In this study, the influence of membrane tension to the process of membrane fusion was investigated with two experimental approaches. At first, GUVs with an adjustable adhesion area were imaged with a confocal microscope to receive a three dimensional image stack for the calculation of membrane tension through the measurement of the vesicle and adhesion radius on the surface. The second experiment consisted of a developed milli-fluidic device with a thin and stretchable PDMS sheet. On the surface of the thin PDMS sheet, GUVs were spread to form membrane patches with defined and measureable areas. The remaining uncovered PDMS surface was passivated to prevent spreading of the incubated LUVs. With both techniques, membrane tension of the GUVs and SLBs could be adjusted. The membrane tension of the GUVs was elevated by increasing the adhesion area of the GUV-membrane to the substrate. Stretching of the PDMS-substrate led to an increased area of the SLBs on the surface of the PDMS stretching device. The anisotropy of the surface extension on the PDMS-substrate was an intended property of the thin PDMS sheet because many different area changes of SLBs could be measured in the same sample and thereby a large variety of adjusted membrane tensions.

The minimal fusion machinery consisting of syntaxin, SNAP 25 and synaptobrevin, as described previously, was utilized to obtain a reasonable fusion efficiency to begin with. ${ }^{24,30}$ For the measurements in this thesis, lipids with the headgroup phosphatidylethanolamine (PE) were used that are known to result in negative spontaneous curvature in membranes and thus promote fusion processes. In regulated exocytosis of secretory vesicles cholesterol is concentrated in the membrane and has a major impact on vesicle fusion by clustering of the SNAREs and the formation and stability of fusion pores. ${ }^{75-76}$ Without cholesterol, most vesicles become arrested at hemi-fusion whereas an increased amount of cholesterol (30-40 mol\%) results in full fusion of vesicles..$^{75}$ In the performed measurements for this thesis the vesicles contained $11 \mathrm{~mol} \%$ of cholesterol and mostly full fusion of vesicles to the tensed SLBs and GUVs was found but also some hemi-fusion vesicles if the area expansion of the SLBs is exhausted. In the absence of fusogenic proteins, lipid bilayers do not show a significant number of fusion events in the experimental time frame of around 30 to $50 \mathrm{~min}$ regardless of the applied tension. In both experimental setups unstressed SLBs or slightly adhered GUVs equipped with SNAREs show only few docked LUVs.

The LUV fusion experiments on adhered GUVs was an approach to measure LUV fusion through content and lipid mixing. Because of the 3D-geometry of the adhered GUV on the substrate surface the buffer solution with the added LUVs floated around the GUV so that the collision of the LUVs with the target membrane of the GUV could occur in all three dimensions whereas the collision between the SLB and the LUVs was only achieved by the LUVs with a movement along the vertical axis. In comparison, synaptic vesicles in neurons are directly tethered through a network of proteins to the active zone. The GUV-LUV fusion experiment depended on the added LUV concentration and diffusion of the LUVs to the adhered GUVS because of the large buffer volume of $3-5 \mathrm{~mL}$. The adhered GUVs on the surface also tend to rupture and spread on the surface whereas the SLBs on the PDMS surface were stable and fixed at their position. Evaporation of water from the buffer solution was prevented by placing the whole 
sample, containing the adhered GUVs, under a sealed dome with constant water vapor pressure. Nonetheless a small difference in osmotic condition between the content solution and the surrounding buffer solution of the GUV could not be ruled out.

In the content mixing assay, described in chapter 5.1.2, the largest GUV at the smallest membrane tension of $0.12 \mathrm{mN} / \mathrm{m}$ showed no fluorescence from the dye ATTO $^{\circledR} 488$ that was encapsulated in the LUVs. The smallest GUV with a membrane tension of $0.94 \mathrm{mN} / \mathrm{m}$ showed the same fluorescence intensity as the surrounding buffer solution and thus reports that increased membrane tension leads to a higher fusion efficiency. One has to consider that the smallest GUV has a much smaller volume compared to the largest GUV. This dilution effect should be diminished over time but the largest GUV did not show and fluorescence intensity increase at all. FRAP-measurements of the small GUV-content show that LUV fusion did not stop and the fluorescence intensity recovers slowly after some time so that LUV fusion and content mixing is the only possible reason for that. A sample without SNAREs but highly tensed GUVs showed no fluorescence increase at the content of the GUVs. A diffusion of the water soluble dye from the surrounding buffer or uncontrolled LUV fusion could not be detected. Therefore, the lipid mixing assay proved that membrane fusion occurs at a membrane tensions of $0.29 \mathrm{mN} / \mathrm{m}$ and $0.94 \mathrm{mN} / \mathrm{m}$ which is slightly higher than tension of many plasma membranes of cells. A small amount of fused LUVs could lead to the fluorescence intensity increase inside the GUVs so that this content mixing experiment is a proof of fully fused membranes between the GUV and the LUVs using SNAREs as fusion promotes.

For the lipid mixing assay, 24 GUVs were measured to calculate the membrane tensions and to compare the fluorescence intensities of the LUV dye at the freestanding GUV-membrane. FRAP-measurements at the adhesion site of the GUVs were used to determine the lipid mixing of the LUVs with GUV. The result of this lipid mixing assay shows that an elevated membrane tension is a crucial property for the amount of docked and fused LUVs. At low membrane tension below $0.6 \mathrm{mN} / \mathrm{m}$ only a few LUVs dock to the freestanding GUV-membrane and LUV fusion is not detectable (see figure 5.2-5.4). At a membrane tension of around $0.8 \mathrm{mN} / \mathrm{m}$ the docking rate of the LUVs to the GUV appreciably increased and many peak intensities, representing LUVs or clusters of LUVs, could be detected (see figure 5.5). Higher membrane tensions than $1.5 \mathrm{mN} / \mathrm{m}$ showed a higher LUV fusion efficiency (figure 5.6-5.8). LUV fusion was detected through a fluorescence intensity increase of the LUV dye at the adhesion site of the GUV. The lipid dye, originating from the LUVs, freely diffuses in the membrane after fusion which was proven by FRAP-measurements (see figures 5.11-5.13). In one experiment, it was possible to measure two different GUVs as an intrinsic comparison, one with a ruptured membrane at the adhesion site, corresponding to a low membrane tension, and one with a large adhesion area, corresponding to high membrane tension (Figure 5.10). The ruptured GUV contained a much lower fluorescence intensity at the adhesion site representing a low fusion efficiency compared to the GUV with a higher membrane tension of $4.8 \mathrm{mN} / \mathrm{m}$. A few LUVs dock to the GUV with low membrane tension whereas the amount of docked LUVs at surface of the tensed GUV-membranes is greater. As a result, the lipid mixing assay with adhered GUVs on a functionalized glass surface proved the hypothesis that elevated membrane tension on the GUVs increases the amount of docked and fused LUVs. 
The second experimental approach was used to further prove the hypothesis that elevated membrane tension increases membrane fusion ${ }^{55}$ through a direct measure of the membrane area change. The membrane tension could be calculated for each area increase from equation (6) described in chapter 2 . At first, the constructed and fabricated milli-fluidic device was tested to prove that it is possible to spread supported lipid bilayer on the surface of the thin PDMS sheet as previously described by STAYKOVA et al. ${ }^{58}$ Spreading of LUVs on the hydrophilic PDMS surface leads to the formation of a fully covered supported lipid bilayer that could be stretched and compressed through a lower or higher air pressure in the side channels next to the spanned PDMS sheet. In figure 5.18, a thin PDMS surface covered with a SLB could be stretched until cracks in the membrane occurred but also be compressed so that membrane tubes were formed representing the excess membrane area. With the milli-fluidic device the stretching and compression of membranes was confirmed. Spreading of GUVs on the hydrophilic PDMS surface of the membrane stretcher device yields membrane patches with a defined area. The images of the membrane patches were analyzed by the thresholding technique of $\mathrm{LI}$ et al. to precisely measure the actual membrane area of each membrane patches. ${ }^{62}$ With this thresholding technique the change in membrane patch area through PDMS surface dilatation was proven exemplary by the measurement of a large membrane patch shown in figures 4.6 and 5.32 with an area change of $1.44 \%$ and a corresponding membrane tension of $4 \mathrm{mN} / \mathrm{m}$. Depending on the size of the membrane patches, the average error of the membrane patch area determination was calculated to be around $\Delta\left|\Delta A / A_{0}\right|=(0.38 \pm 0.19) \%$.

Interestingly, it was possible to detect holes in some membrane patches that occurred after surface dilatation of the substrate. In figure 5.25 and 5.28 five membrane patches are shown at membrane tension of zero to $10 \mathrm{mN} / \mathrm{m}$. For membrane patches, containing holes, no area change could be measured. STUBBINGTON et al. published that the time scale of oxidizing a PDMS surface alters the hydrophilicity of the surface. Three seconds of oxidation in the plasma led to a partly hydrophilic surface whereas $30 \mathrm{~s}$ of plasma-oxidation led to strong hydrophilic surface. ${ }^{59}$ Adhered membrane patches on both differently oxidized surfaces showed upon surface dilatation membrane area change (sticking) and cracks on the partly hydrophilic surface and sliding on the strong hydrophilic PDMS surface. Here, the used PDMS surfaces of the membrane stretcher device were oxidized in a plasma generator for $10 \mathrm{~s}$ which is in between the oxidation time of STUBBINGTON et al.. The measurements for this thesis confirmed that membrane patches on a stretched PDMS surface could stick and rupture through surface dilatation. But also sliding of membrane patches with no area change occurred. Besides, holes frequently appeared on many membrane patches in response to stretching of the underlying substrate. As a consequence of the erupted holes, the membrane area stays nearly constant compared to its initial area in the relaxed state. The most important scenario is that the membrane follows the expansion of the PDMS substrate to induce lateral membrane tension.

The hydrophilic PDMS surface around the membrane patches induced spreading of LUVs and lipid mixing with SLBs spread from GUVs (Figure 5.19). Therefore, the hydrophilic PDMS surface was passivated with the protein BSA comparable to the hydrophilic glass surface in the GUV-adhesion experiment. The passivated control sample without using SNAREs as fusion promoters in figure 5.20 
showed no docking and fusion of LUVs to the membrane patches so that undefined fusion of LUVs on the SLBs can be excluded.

Unstressed membranes equipped with SNARE proteins show only few fusion events, while membrane tension above $2.8-5.5 \mathrm{mN} / \mathrm{m}$ corresponding to a relative area increase of $1-2 \%$ increases fusion efficiency substantially by $50 \%$ which is in agreement with the fusion experiment with adhered GUVs. However, an elevation of membrane tension above the lysis tension of $10 \mathrm{mN} / \mathrm{m}$ leads to a drop of the relative fusion efficiency (Figure 5.34).

A direct comparison of a SLB at a membrane tension of $(4.4 \pm 0.8) \mathrm{mN} / \mathrm{m}$ with a membrane patch at a membrane tension of $(1.0 \pm 0.7) \mathrm{mN} / \mathrm{m}$ in the same sample, depicted in figure 5.30 and 5.31 , confirms the hypothesis of SHILLCOCK and LIPOWSKI that an elevated membrane tension results in an increased fusion probability. ${ }^{55}$

In essence, a maximal fusion efficiency $F_{\text {eff }}$ for the membrane tension is found in the range of $5-10 \mathrm{mN} / \mathrm{m}$ (Figure 5.34 and 5.35). This observation is in good accordance with a theoretical study of GRAFMÜLLER et al. using dissipative particle simulations. ${ }^{114}$ The authors found that fusion probability is maximal at intermediate tensions. While membrane rupture limits fusion probability at very large tensions, low tensions do not lower the energy barrier of fusion sufficiently since a relaxed planar bilayer has not enough space for lipids pouring in from LUVs. At low tension, either the hemi-fused patch might expand and gain membrane area and thereby relax the membrane tension or the adhering state might remain stable. GRAFMÜLLER et al. found that the fusion process consists of at least three consecutive steps in which the first two steps are tension dependent: (1) Interbilayer flips of lipid tails, (2) nucleation of a small hemi-fused area and (3) pore formation. Interbilayer flip and nucleation of hemi-fusion depend both exponentially on the tension. It is conceivable that the hydrophobic contact is favored at intermediate tension facilitating interbilayer exchange (lipid splay) of lipids in the opposing membranes. Using simulations that enforce interbilayer flips of individual lipid tails and utilizing Jarzynski's relation, GRAFMÜLLER et al. determined the energy scale for these barriers. ${ }^{114}$

According to Kozlov and Chernomordik three major origins of forces acting on cell membranes and generating membrane tension exist: (1) osmotic pressure, i.e., a difference in hydrostatic pressure between the cytosol and the external medium; (2) membrane adhesion to the actomyosin cortex and (3) cellular adhesion and subsequent spreading on substrates or other cells. ${ }^{44}$ The characteristic tensions found in the plasma membrane of eukaryotic cells are only in the range of $0.01-1 \mathrm{mN} / \mathrm{m}$ depending on the cell type. Therefore, typical tension values found in the plasma membrane of cells are maybe not sufficient to substantially increase the fusion efficiency. This is rather expected, considering the fact that unwanted fusion needs to be suppressed in non-specialized cells. However, the tensions at the sites of exocytosis in the presynaptic membrane are not known. Proteins from the active zone could in principle enhance fusion probability by increasing tension locally very close to rupture tension. Such increased tension would need to be locally constrained since tension inhibits clathrin mediated endocytosis of synaptic vesicles. Recently in a more biological study with cells Wen et al. found that dynamic assembly of filamentous actin including also ATP hydrolysis, mediates $\Omega$-profile merging by providing sufficient plasma membrane tension to shrink the $\Omega$-profile in neuroendocrine chromaffin cells containing $\sim 300 \mathrm{~nm}$ vesicles. ${ }^{47} \mathrm{It}$ is clear that mechanical tension plays a pivotal role in vesicle trafficking and it will 
be interesting to see in the future how cells regulate tension to adjust the energy landscape of these processes in order to control their kinetics.

The key finding of this thesis is that the probability of membrane fusion increases when external tension is applied to one of the participating membranes. Here, the membrane tension was gradually increased through an area expansion of the adhesion site of the GUVs and through the dilatation of the PDMSsubstrate for the SLBs. In the range of the applied membrane tension between $0.1-10 \mathrm{mN} / \mathrm{m}$ the fusion efficiency of LUVs on SLBs and GUVs was analyzed. In both experiments a threshold tension of $3.4 \mathrm{mN} / \mathrm{m}$ was detected at which the amount of docked and fused LUVs to the tensed target membrane was boosted. As a conclusion, the hypothesis of a tension-dependency of vesicle fusion is confirmed. 


\section{Conclusion}

The large gap in time scales between membrane fusion occurring in biological systems during neurotransmitter release and fusion observed between model membranes equipped with fusogenic proteins has led to a large number of possible factors that might explain this discrepancy. One possible reason for faster and more efficient fusion in native systems compared to model membranes is an elevated lateral membrane tension present in the presynaptic membrane. Theoretical predictions and computer simulations suggested that intermediate membrane tension might reduce energy barriers of membrane merging and increase the probability of fusion. A direct experimental evidence for these claims did not exist until now. However, it is entirely unknown to what extent a change in lateral tension influences fusion efficiency in the presence of SNAREs. Here, two new methods are devised to increase the membrane tension of adhered GUVs and isolated membrane patches derived from GUVs that are spread on a stretchable PDMS sheet. With these two systems, the membrane tension of the GUVs and SLBs is adjustable and the tension-dependency of fusion using this model membranes equipped with a minimal fusion machinery consisting of syntaxin 1, synaptobrevin and SNAP 25 was investigated.

Adhesion of giant vesicles on a functionalized glass surface was increased through the addition of divalent ions into the buffer solution to adjust a certain membrane tension through an increased adhesion area of the GUVs containing syntax 1 and preassembled SNAP 25 ( $\Delta \mathrm{N}$-complex). Isolated patches of planar bilayers containing the $\Delta \mathrm{N} 49$-complex were formed from giant unilamellar vesicles and deposited on a dilatable PDMS sheet, which is part of a milli-fluidic chamber allowing to adjust lateral tension in bilayer patches. Docking, hemi-fusion and fusion of large unilamellar vesicles functionalized with synaptobrevin were followed by fluorescence microscopy. The relative increase in fluorescence intensity of ATTO 594 (red), originating from the added LUVs, compared to the fluorescence intensity of ATTO 390 (blue), emitted from the planar bilayer patch, served as a measure for fusion efficiency.

It was found that fusion efficiency increases considerably with lateral tension and a threshold tension of $3.4 \mathrm{mN} / \mathrm{m}$ in both experiments was identified at which fusion is boosted tremendously, which is attributed to facilitation of lipid splaying and assisted fusion pore expansion. The results prove experimentally the theoretically predicted hypothesis that membrane tension steers membrane fusion and might therefore be an essential prerequisite for fast fusion as observed for the release of neurotransmitters at the neuronal synapse. The implications of this thesis are that local tension needs to be substantial, even close to bilayer lysis to increase fusion efficiency with unstressed vesicles. 


\section{References}

1. Hay, J. C.; Chao, D. S.; Kuo, C. S.; Scheller, R. H., Protein Interactions Regulating Vesicle Transport between the Endoplasmic Reticulum and Golgi Apparatus in Mammalian Cells. Cell 1997, 89 (1), 149-158.

2. Martens, S.; McMahon, H. T., Mechanisms of membrane fusion: disparate players and common principles. Nat Rev Mol Cell Biol 2008, 9 (7), 543-556.

3. Scheller, R. H., In search of the molecular mechanism of intracellular membrane fusion and neurotransmitter release. Nat Med 2013, 19 (10), 1232-1235.

4. Smirnova, Y. G.; Aeffner, S.; Risselada, H. J.; Salditt, T.; Marrink, S. J.; Muller, M.; Knecht, V., Interbilayer repulsion forces between tension-free lipid bilayers from simulation. Soft Matter 2013, 9 (45), 10705-10718.

5. Risselada, H. J.; Grubmüller, H., How SNARE molecules mediate membrane fusion: Recent insights from molecular simulations. Current Opinion in Structural Biology 2012, 22 (2), 187196.

6. Wiederhold, K.; Fasshauer, D., Is Assembly of the SNARE Complex Enough to Fuel Membrane Fusion? Journal of Biological Chemistry 2009, 284 (19), 13143-13152.

7. Li, F.; Pincet, F.; Perez, E.; Eng, W. S.; Melia, T. J.; Rothman, J. E.; Tareste, D., Energetics and dynamics of SNAREpin folding across lipid bilayers. Nat Struct Mol Biol 2007, 14 (10), 890-896.

8. Sutton, R. B.; Fasshauer, D.; Jahn, R.; Brunger, A. T., Crystal structure of a SNARE complex involved in synaptic exocytosis at 2.4[thinsp]A resolution. Nature 1998, 395 (6700), 347-353.

9. Hernandez, J. M.; Stein, A.; Behrmann, E.; Riedel, D.; Cypionka, A.; Farsi, Z.; Walla, P. J.; Raunser, S.; Jahn, R., Membrane Fusion Intermediates via Directional and Full Assembly of the SNARE Complex. Science 2012, 336 (6088), 1581.

10. Südhof, Thomas C., Neurotransmitter Release: The Last Millisecond in the Life of a Synaptic Vesicle. Neuron 2013, 80 (3), 675-690.

11. Takamori, S.; Holt, M.; Stenius, K.; Lemke, E. A.; Grønborg, M.; Riedel, D.; Urlaub, H.; Schenck, S.; Brügger, B.; Ringler, P.; Müller, S. A.; Rammner, B.; Gräter, F.; Hub, J. S.; De Groot, B. L.; Mieskes, G.; Moriyama, Y.; Klingauf, J.; Grubmüller, H.; Heuser, J.; Wieland, F.; Jahn, R., Molecular Anatomy of a Trafficking Organelle. Cell 2006, 127 (4), 831-846.

12. Südhof, Thomas C., The Presynaptic Active Zone. Neuron 2012, 75 (1), 11-25.

13. Zhai, R. G.; Bellen, H. J., The Architecture of the Active Zone in the Presynaptic Nerve Terminal. Physiology 2004, 19 (5), 262.

14. Watanabe, S.; Rost, B. R.; Camacho-Perez, M.; Davis, M. W.; Sohl-Kielczynski, B.; Rosenmund, C.; Jorgensen, E. M., Ultrafast endocytosis at mouse hippocampal synapses. Nature 2013, 504 (7479), 242-247.

15. Südhof, T. C., THE SYNAPTIC VESICLE CYCLE. Annual Review of Neuroscience 2004, 27 (1), 509-547.

16. Alabi, A. A.; Tsien, R. W., Perspectives on Kiss-and-Run: Role in Exocytosis, Endocytosis, and Neurotransmission. Annual Review of Physiology 2013, 75 (1), 393-422.

17. McMahon, H. T.; Boucrot, E., Molecular mechanism and physiological functions of clathrinmediated endocytosis. Nat Rev Mol Cell Biol 2011, 12 (8), 517-533.

18. Saheki, Y.; De Camilli, P., Synaptic Vesicle Endocytosis. Cold Spring Harbor Perspectives in Biology 2012, 4 (9).

19. Mittelstaedt, T.; Alvaréz-Baron, E.; Schoch, S., RIM proteins and their role in synapse function. In Biological Chemistry, 2010; Vol. 391, p 599.

20. Vrljic, M.; Strop, P.; Ernst, J. A.; Sutton, R. B.; Chu, S.; Brunger, A. T., Molecular mechanism of the synaptotagmin-SNARE interaction in Ca2+-triggered vesicle fusion. Nat Struct Mol Biol 2010, 17 (3), 325-331.

21. Chen, Y. A.; Scales, S. J.; Patel, S. M.; Doung, Y.-C.; Scheller, R. H., SNARE Complex Formation Is Triggered by Ca2+ and Drives Membrane Fusion. Cell 1999, 97 (2), 165-174.

22. Jahn, R.; Scheller, R. H., SNAREs - engines for membrane fusion. Nat Rev Mol Cell Biol 2006, $7(9), 631-643$.

23. Lin, C.-C.; Seikowski, J.; Pérez-Lara, A.; Jahn, R.; Höbartner, C.; Walla, P. J., Control of membrane gaps by synaptotagmin-Ca2+ measured with a novel membrane distance ruler. Nature Communications 2014, 5, 5859. 
24. Schwenen, L. L. G.; Hubrich, R.; Milovanovic, D.; Geil, B.; Yang, J.; Kros, A.; Jahn, R.; Steinem, C., Resolving single membrane fusion events on planar pore-spanning membranes. Scientific Reports 2015, 5, 12006.

25. Wang, T.; Smith, E. A.; Chapman, E. R.; Weisshaar, J. C., Lipid Mixing and Content Release in Single-Vesicle, SNARE-Driven Fusion Assay with 1-5 ms Resolution. Biophysical Journal 2009, 96 (10), 4122-4131.

26. van den Bogaart, G.; Holt, M. G.; Bunt, G.; Riedel, D.; Wouters, F. S.; Jahn, R., One SNARE complex is sufficient for membrane fusion. Nat Struct Mol Biol 2010, 17 (3), 358-364.

27. Aeffner, S.; Reusch, T.; Weinhausen, B.; Salditt, T., Energetics of stalk intermediates in membrane fusion are controlled by lipid composition. Proceedings of the National Academy of Sciences 2012, 109 (25), E1609-E1618.

28. Chernomordik, L. V.; Kozlov, M. M., Mechanics of membrane fusion. Nat Struct Mol Biol 2008, $15(7), 675-683$.

29. Savić, F.; Kliesch, T.-T.; Verbeek, S.; Bao, C.; Thiart, J.; Kros, A.; Geil, B.; Janshoff, A., Geometry of the Contact Zone between Fused Membrane-Coated Beads Mimicking Cell-Cell Fusion. Biophysical Journal 2016, 110 (10), 2216-2228.

30. Oelkers, M.; Witt, H.; Halder, P.; Jahn, R.; Janshoff, A., SNARE-mediated membrane fusion trajectories derived from force-clamp experiments. Proceedings of the National Academy of Sciences 2016, 113 (46), 13051-13056.

31. Ryham, Rolf J.; Klotz, Thomas S.; Yao, L.; Cohen, Fredric S., Calculating Transition Energy Barriers and Characterizing Activation States for Steps of Fusion. Biophysical Journal 2016, $110(5), 1110-1124$.

32. Kawamoto, S.; Shinoda, W., Free energy analysis along the stalk mechanism of membrane fusion. Soft Matter 2014, 10 (17), 3048-3054.

33. François-Martin, C.; Rothman, J. E.; Pincet, F., Low energy cost for optimal speed and control of membrane fusion. Proceedings of the National Academy of Sciences 2017, 114 (6), 12381241.

34. Domanska, M. K.; Kiessling, V.; Tamm, L. K., Docking and Fast Fusion of Synaptobrevin Vesicles Depends on the Lipid Compositions of the Vesicle and the Acceptor SNARE ComplexContaining Target Membrane. Biophysical Journal 2010, 99 (9), 2936-2946.

35. McMahon, H. T.; Kozlov, M. M.; Martens, S., Membrane Curvature in Synaptic Vesicle Fusion and Beyond. Cell 2010, 140 (5), 601-605.

36. Hui, E.; Johnson, C. P.; Yao, J.; Dunning, F. M.; Chapman, E. R., Synaptotagmin-Mediated Bending of the Target Membrane Is a Critical Step in Ca2+-Regulated Fusion. Cell 2009, 138 (4), 709-721.

37. Helfrich, W., Elastic Properties of Lipid Bilayers: Theory and Possible Experiments. In Zeitschrift für Naturforschung C, 1973; Vol. 28, p 693.

38. Pietuch, A.; Brückner, B. R.; Janshoff, A., Membrane tension homeostasis of epithelial cells through surface area regulation in response to osmotic stress. Biochimica et Biophysica Acta (BBA) - Molecular Cell Research 2013, 1833 (3), 712-722.

39. Diz-Muñoz, A.; Fletcher, D. A.; Weiner, O. D., Use the force: membrane tension as an organizer of cell shape and motility. Trends in Cell Biology 2013, 23 (2), 47-53.

40. Kozlov, M. M.; McMahon, H. T.; Chernomordik, L. V., Protein-driven membrane stresses in fusion and fission. Trends in Biochemical Sciences 2010, 35 (12), 699-706.

41. Baumgart, T.; Capraro, B. R.; Zhu, C.; Das, S. L., Thermodynamics and Mechanics of Membrane Curvature Generation and Sensing by Proteins and Lipids. Annual Review of Physical Chemistry 2011, 62 (1), 483-506.

42. Gauthier, N. C.; Masters, T. A.; Sheetz, M. P., Mechanical feedback between membrane tension and dynamics. Trends in Cell Biology 2012, 22 (10), 527-535.

43. Paluch, E. K.; Nelson, C. M.; Biais, N.; Fabry, B.; Moeller, J.; Pruitt, B. L.; Wollnik, C.; Kudryasheva, G.; Rehfeldt, F.; Federle, W., Mechanotransduction: use the force(s). BMC Biology 2015, $13(1), 47$.

44. Kozlov, M. M.; Chernomordik, L. V., Membrane tension and membrane fusion. Current Opinion in Structural Biology 2015, 33, 61-67.

45. Jackman, J. A.; Choi, J.-H.; Zhdanov, V. P.; Cho, N.-J., Influence of Osmotic Pressure on Adhesion of Lipid Vesicles to Solid Supports. Langmuir 2013, 29 (36), 11375-11384.

46. Gleisner, M.; Mey, I.; Barbot, M.; Dreker, C.; Meinecke, M.; Steinem, C., Driving a planar model system into the 3rd dimension: generation and control of curved pore-spanning membrane arrays. Soft Matter 2014, 10 (33), 6228-6236. 
47. Wen, P. J.; Grenklo, S.; Arpino, G.; Tan, X.; Liao, H.-S.; Heureaux, J.; Peng, S.-Y.; Chiang, H.C.; Hamid, E.; Zhao, W.-D.; Shin, W.; Näreoja, T.; Evergren, E.; Jin, Y.; Karlsson, R.; Ebert, S. N.; Jin, A.; Liu, A. P.; Shupliakov, O.; Wu, L.-G., Actin dynamics provides membrane tension to merge fusing vesicles into the plasma membrane. Nature Communications 2016, 7, 12604.

48. Pietuch, A.; Janshoff, A., Mechanics of spreading cells probed by atomic force microscopy. Open Biology 2013, 3 (7).

49. Gleisner, M.; Kroppen, B.; Fricke, C.; Teske, N.; Kliesch, T.-T.; Janshoff, A.; Meinecke, M.; Steinem, C., Epsin N-terminal Homology Domain (ENTH) Activity as a Function of Membrane Tension. Journal of Biological Chemistry 2016, 291 (38), 19953-19961.

50. Evans, E.; Skalak, R., Mechanics and Thermodynamics of Biomembranes. CRC Press: Boca Raton, FL: 1980.

51. Rawicz, W.; Olbrich, K. C.; McIntosh, T.; Needham, D.; Evans, E., Effect of Chain Length and Unsaturation on Elasticity of Lipid Bilayers. Biophysical Journal 2000, 79 (1), 328-339.

52. Schneider, D.; Baronsky, T.; Pietuch, A.; Rother, J.; Oelkers, M.; Fichtner, D.; Wedlich, D.; Janshoff, A., Tension Monitoring during Epithelial-to-Mesenchymal Transition Links the Switch of Phenotype to Expression of Moesin and Cadherins in NMuMG Cells. PLOS ONE 2013, 8 (12), e80068.

53. Brückner, B. R.; Pietuch, A.; Nehls, S.; Rother, J.; Janshoff, A., Ezrin is a Major Regulator of Membrane Tension in Epithelial Cells. Scientific Reports 2015, 5, 14700.

54. Schäfer, E.; Kliesch, T.-T.; Janshoff, A., Mechanical Properties of Giant Liposomes Compressed between Two Parallel Plates: Impact of Artificial Actin Shells. Langmuir 2013, 29 (33), 1046310474.

55. Shillcock, J. C.; Lipowsky, R., Tension-induced fusion of bilayer membranes and vesicles. Nat Mater 2005, 4 (3), 225-228.

56. Bretou, M.; Jouannot, O.; Fanget, I.; Pierobon, P.; Larochette, N.; Gestraud, P.; Guillon, M.; Emiliani, V.; Gasman, S.; Desnos, C.; Lennon-Duménil, A.-M.; Darchen, F., Cdc42 controls the dilation of the exocytotic fusion pore by regulating membrane tension. Molecular Biology of the Cell 2014, 25 (20), 3195-3209.

57. Kim, Ji H.; Ren, Y.; Ng, Win P.; Li, S.; Son, S.; Kee, Y.-S.; Zhang, S.; Zhang, G.; Fletcher, Daniel A.; Robinson, Douglas N.; Chen, Elizabeth H., Mechanical Tension Drives Cell Membrane Fusion. Developmental Cell 2015, 32 (5), 561-573.

58. Staykova, M.; Holmes, D. P.; Read, C.; Stone, H. A., Mechanics of surface area regulation in cells examined with confined lipid membranes. Proceedings of the National Academy of Sciences 2011, 108 (22), 9084-9088.

59. Stubbington, L.; Arroyo, M.; Staykova, M., Sticking and sliding of lipid bilayers on deformable substrates. Soft Matter 2016.

60. Huh, D.; Hamilton, G. A.; Ingber, D. E., From 3D cell culture to organs-on-chips. Trends in Cell Biology 2011, 21 (12), 745-754.

61. Huh, D.; Matthews, B. D.; Mammoto, A.; Montoya-Zavala, M.; Hsin, H. Y.; Ingber, D. E., Reconstituting Organ-Level Lung Functions on a Chip. Science 2010, 328 (5986), 1662.

62. Li, C. H.; Tam, P. K. S., An iterative algorithm for minimum cross entropy thresholding. Pattern Recognition Letters 1998, 19 (8), 771-776.

63. Lipowsky, R., Coupling of bending and stretching deformations in vesicle membranes. Advances in Colloid and Interface Science 2014, 208, 14-24.

64. Boal, D., Mechanics of the cell. Second Edition ed.; Cambridge University Press, The Edinburgh Building, Cambridge, UK: New York, 2012.

65. Evans, E.; Rawicz, W., Entropy-driven tension and bending elasticity in condensed-fluid membranes. Physical Review Letters 1990, 64 (17), 2094-2097.

66. ATTO-TEC Labeled Phospholipids: https://www.atto-tec.com/. (accessed 04.01.2017).

67. Shi, X.; Halder, P.; Yavuz, H.; Jahn, R.; Shuman, H. A., Direct targeting of membrane fusion by SNARE mimicry: Convergent evolution of Legionella effectors. Proceedings of the National Academy of Sciences 2016, 113 (31), 8807-8812.

68. Sekar, R. B.; Periasamy, A., Fluorescence resonance energy transfer (FRET) microscopy imaging of live cell protein localizations. The Journal of Cell Biology 2003, 160 (5), 629.

69. Clegg, R. M., Fluorescence resonance energy transfer. Current Opinion in Biotechnology 1995, $6(1), 103-110$.

70. Marsh, D., Handbook of Lipid Bilayers. Second Edition ed.; CRC Press Taylor \& Francis Group: 6000 Broken Sound Parkway NW, Boca Raton, FL, 2013. 
71. Churchward, M. A.; Rogasevskaia, T.; Brandman, D. M.; Khosravani, H.; Nava, P.; Atkinson, J. K.; Coorssen, J. R., Specific Lipids Supply Critical Negative Spontaneous Curvature-An Essential Component of Native Ca2+-Triggered Membrane Fusion. Biophysical Journal 2008, 94 (10), 3976-3986.

72. Mondal Roy, S.; Sarkar, M., Membrane Fusion Induced by Small Molecules and Ions. Journal of Lipids 2011, 2011, 528784.

73. Marsden, H. R.; Tomatsu, I.; Kros, A., Model systems for membrane fusion. Chem Soc Rev 2011, 40 (3), 1572-85.

74. Dowhan, W.; Bogdanov, M., Chapter 1 Functional roles of lipids in membranes. In New Comprehensive Biochemistry, Elsevier: 2002; Vol. Volume 36, pp 1-35.

75. Kreutzberger, Alex J. B.; Kiessling, V.; Tamm, Lukas K., High Cholesterol Obviates a Prolonged Hemifusion Intermediate in Fast SNARE-Mediated Membrane Fusion. Biophysical Journal 2015, 109 (2), 319-329.

76. Yang, S.-T.; Kreutzberger, A. J. B.; Lee, J.; Kiessling, V.; Tamm, L. K., The role of cholesterol in membrane fusion. Chemistry and Physics of Lipids 2016, 199, 136-143.

77. Murphy, D. B., Fundamentals of Light Microscopy and Electronic Imaging. Wiley-Liss, Inc.: USA, 2001.

78. Texas Red® 1,2-Dihexadecanoyl-sn-Glycero-3-Phosphoethanolamine, Triethylammonium Salt (Texas Red® DHPE). https://www.thermofisher.com/order/catalog/product/T1395MP (accessed 10.01.2017).

79. ATTO-TEC, Fluorescent Labels and Dyes. In Product Catalogue, ATTO-TEC GmbH: 2013/2015.

80. Lazzara, T. D.; Behn, D.; Kliesch, T.-T.; Janshoff, A.; Steinem, C., Phospholipids as an alternative to direct covalent coupling: Surface functionalization of nanoporous alumina for protein recognition and purification. Journal of Colloid and Interface Science 2012, 366 (1), 57 63.

81. Lazzara, T. D.; Kliesch, T.-T.; Janshoff, A.; Steinem, C., Orthogonal Functionalization of Nanoporous Substrates: Control of 3D Surface Functionality. ACS Applied Materials \& Interfaces 2011, 3 (4), 1068-1076.

82. Walde, P.; Cosentino, K.; Engel, H.; Stano, P., Giant Vesicles: Preparations and Applications. ChemBioChem 2010, 11 (7), 848-865.

83. Fenz, S. F.; Sengupta, K., Giant vesicles as cell models. Integrative Biology 2012, 4 (9), $982-$ 995.

84. Angelova, M. I.; Dimitrov, D. S., Liposome electroformation. Faraday Discussions of the Chemical Society 1986, 81 (0), 303-311.

85. Schäfer, E.; Vache, M.; Kliesch, T.-T.; Janshoff, A., Mechanical response of adherent giant liposomes to indentation with a conical AFM-tip. Soft Matter 2015, 11 (22), 4487-4495.

86. Akashi, K.; Miyata, H.; Itoh, H.; Kinosita, K., Preparation of giant liposomes in physiological conditions and their characterization under an optical microscope. Biophysical Journal 1996, 71 (6), 3242-3250.

87. Montes, L. R.; Alonso, A.; Goñi, F. M.; Bagatolli, L. A., Giant Unilamellar Vesicles Electroformed from Native Membranes and Organic Lipid Mixtures under Physiological Conditions. Biophysical Journal 2007, 93 (10), 3548-3554.

88. Good, N. E.; Winget, G. D.; Winter, W.; Connolly, T. N.; Izawa, S.; Singh, R. M. M., Hydrogen Ion Buffers for Biological Research*. Biochemistry 1966, 5 (2), 467-477.

89. Henriksen, J. R.; Ipsen, J. H., Measurement of membrane elasticity by micro-pipette aspiration. The European Physical Journal E 2004, 14 (2), 149-167.

90. Jeyachandran, Y. L.; Mielczarski, J. A.; Mielczarski, E.; Rai, B., Efficiency of blocking of nonspecific interaction of different proteins by BSA adsorbed on hydrophobic and hydrophilic surfaces. Journal of Colloid and Interface Science 2010, 341 (1), 136-142.

91. Maloney, A.; Herskowitz, L. J.; Koch, S. J., Effects of Surface Passivation on Gliding Motility Assays. PLOS ONE 2011, 6 (6), e19522.

92. Stein, A.; Weber, G.; Wahl, M. C.; Jahn, R., Helical extension of the neuronal SNARE complex into the membrane. Nature 2009, 460 (7254), 525-528.

93. Synaptosomal-associated protein 25. http://www.uniprot.org/uniprot/P60881 (accessed 16.01.2017).

94. Syntaxin-1A. http://www.uniprot.org/uniprot/P32851 (accessed 16.01.2017).

95. Vesicle-associated membrane protein 2. http://www.uniprot.org/uniprot/P63045 (accessed 16.01.2017).

96. Pobbati, A. V.; Stein, A.; Fasshauer, D., N- to C-Terminal SNARE Complex Assembly Promotes Rapid Membrane Fusion. Science 2006, 313 (5787), 673. 
97. Schubert, R., Liposome Preparation by Detergent Removal. In Methods in Enzymology, Academic Press: 2003; Vol. Volume 367, pp 46-70.

98. Pencer, J.; Hallett, F. R., Effects of Vesicle Size and Shape on Static and Dynamic Light Scattering Measurements. Langmuir 2003, 19 (18), 7488-7497.

99. Berne, B. J.; Pecora, R., Dynamic Light Scattering With Applications to Chemistry, Biology, and Physics. John Wiley \& Sons: NY, USA, 2000.

100. Fuard, D.; Tzvetkova-Chevolleau, T.; Decossas, S.; Tracqui, P.; Schiavone, P., Optimization of poly-di-methyl-siloxane (PDMS) substrates for studying cellular adhesion and motility. Microelectronic Engineering 2008, 85 (5-6), 1289-1293.

101. Harwardt, M. M. Funktionalisierung von Polydimethylsiloxan-Oberflächen zur Steuerung molekularer Zell-Substrat Wechselwirkungen. Dissertation, RWTH Aachen, Aachen, 2007.

102. Xia, Y.; McClelland, J. J.; Gupta, R.; Qin, D.; Zhao, X.-M.; Sohn, L. L.; Celotta, R. J.; Whitesides, G. M., Replica molding using polymeric materials: A practical step toward nanomanufacturing. Advanced Materials 1997, 9 (2), 147-149.

103. IUPAC, Compendium of Chemical Terminology Gold Book. International Union of Pure and Applied Chemistry: 2014.

104. Lakowicz, J. R., Principles of Fluorescence Spectroscopy. Third Edition ed.; Springer Science+Business Media: 233 Spring Street, New York, NY 10013, USA, 2006.

105. Sackmann, E.; Merkel, R., Lehrbuch der Biophysik. Wiley-VCH: 2010.

106. Soumpasis, D. M., Theoretical analysis of fluorescence photobleaching recovery experiments. Biophysical Journal 1983, 41 (1), 95-97.

107. General, I. J.; Dragomirova, R.; Meirovitch, H., Absolute Free Energy of Binding of Avidin/Biotin, Revisited. The Journal of Physical Chemistry B 2012, 116 (23), 6628-6636.

108. Fiji is just ImageJ. https://imagej.net/Downloads (accessed 16.02.2017).

109. Li, C. H.; Lee, C. K., Minimum cross entropy thresholding. Pattern Recognition 1993, 26 (4), 617-625.

110. Pähler, G.; Panse, C.; Diederichsen, U.; Janshoff, A., Coiled-Coil Formation on Lipid BilayersImplications for Docking and Fusion Efficiency. Biophysical Journal 2012, 103 (11), 2295-2303.

111. Schuy, S.; Faiss, S.; Yoder, N. C.; Kalsani, V.; Kumar, K.; Janshoff, A.; Vogel, R., Structure and Thermotropic phase Behavior of Fluorinated Phospholipid Bilayers: A combined Attenuated Total Reflection FTIR Spectroscopy and Imaging Ellipsometry Study. The journal of physical chemistry. B 2008, 112 (28), 8250-8256.

112. Milovanovic, D.; Honigmann, A.; Koike, S.; Göttfert, F.; Pähler, G.; Junius, M.; Müllar, S.; Diederichsen, U.; Janshoff, A.; Grubmüller, H.; Risselada, H. J.; Eggeling, C.; Hell, S. W.; van den Bogaart, G.; Jahn, R., Hydrophobic mismatch sorts SNARE proteins into distinct membrane domains. Nature Communications 2015, 6, 5984.

113. Lambertz, C.; Martos, A.; Henkel, A.; Neiser, A.; Kliesch, T.-T.; Janshoff, A.; Schwille, P.; Sönnichsen, C., Single Particle Plasmon Sensors as Label-Free Technique To Monitor MinDE Protein Wave Propagation on Membranes. Nano Letters 2016, 16 (6), 3540-3544.

114. Grafmüller, A.; Shillcock, J.; Lipowsky, R., The Fusion of Membranes and Vesicles: Pathway and Energy Barriers from Dissipative Particle Dynamics. Biophysical Journal 2009, 96 (7), 26582675. 


\section{Appendix}

\subsection{MATLAB scripts for the calculation of GUV membrane tension}

\subsubsection{Calculation of GUV-membrane area change}

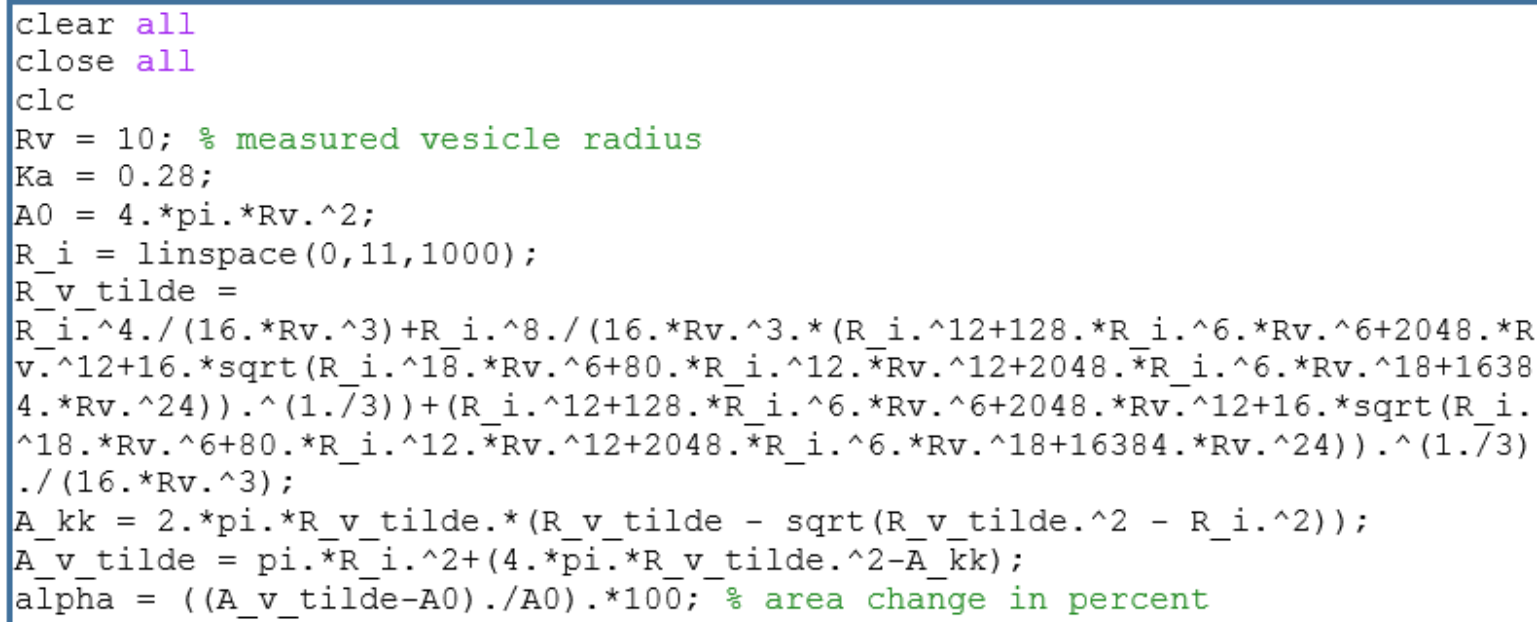




\subsubsection{Calculation of membrane tension as a function of fractional area change}

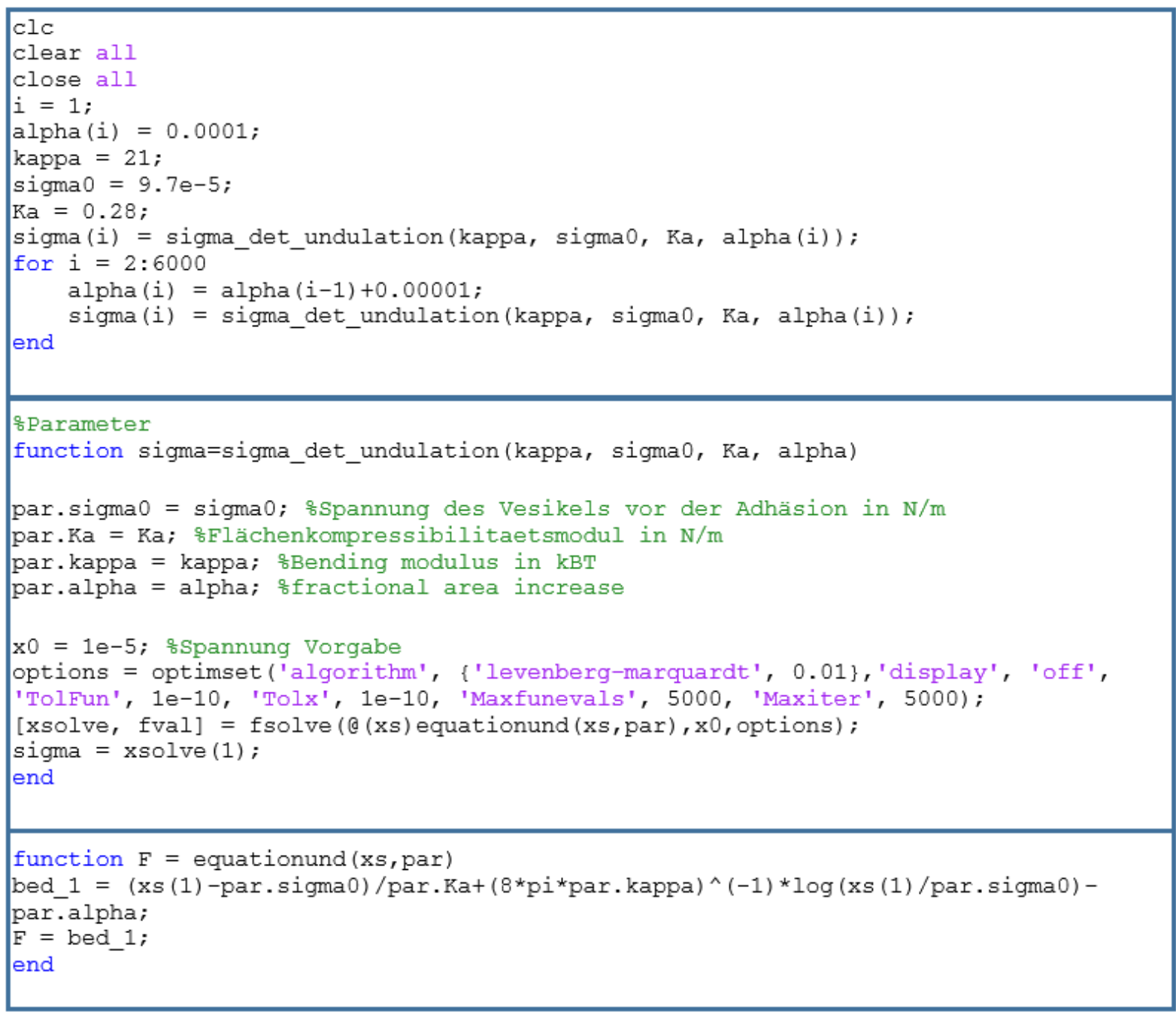




\subsection{Abbreviation}

\begin{tabular}{|c|c|}
\hline a.u. & arbitrary units \\
\hline A390 & ATTO ${ }^{\circledR} 390$ DOPE \\
\hline A488 & ATTO $^{\circledR} 488$ DOPE \\
\hline A594 & ATTO ${ }^{\circledR} 594$ DOPE \\
\hline Av & avidin from egg white \\
\hline BSA & bovine serum albumin \\
\hline $\mathrm{C}_{4} \mathrm{~F}_{8}$ & cyclo-octafluorobutane \\
\hline CAS & casein \\
\hline CHAPS & 3-[(3-Cholamidopropyl)dimethylammonio]-1-propanesulfonate \\
\hline Chol & $3 \beta$-hydroxy-5-cholestene \\
\hline CMC & critical micelle concentration \\
\hline cap-biotinyl-DOPE & 1,2-dioleoyl-sn-glycero-3-phosphoethanolamine- $\mathrm{N}$-(cap biotinyl) \\
\hline CLSM & confocal LASER scanning microscopy \\
\hline DHPE & 1,2-Dihexadecanoyl-sn-Glycero-3-Phosphoethanolamine \\
\hline DLS & dynamic light scattering \\
\hline DOPC & 1,2-dioleoyl-sn-glycero-3-phosphocholine \\
\hline DOPE & 1,2-dioleoyl-sn-glycero-3-phosphoethanolamine \\
\hline DOPS & 1,2-dioleoyl-sn-glycero-3-phospho-L-serine \\
\hline DTT & dithiothreitol \\
\hline$\Delta \mathrm{N}$-complex & syntaxin and SNAP 25 \\
\hline$\Delta$ N49-complex & preassembled syntaxin1A (183-288), SNAP 25 (1-206) and Syb2 (49-96) \\
\hline EDTA & ethylenediaminetetraacetic acid \\
\hline FRAP & fluorescence recovery after photobleaching \\
\hline FRET & förster resonance energy transfer \\
\hline GUV & giant unilamellar vesicle \\
\hline HEPES & 2-[4-(2-hydroxyethyl)piperazin-1-yl]ethanesulfonic acid \\
\hline IPC & Institut für Physikalische Chemie Göttingen \\
\hline $\mathrm{KCl}$ & potassium chloride \\
\hline $\mathrm{KH}_{2} \mathrm{PO}_{4}$ & potassium dihydrogenphosphate \\
\hline LASER & light amplification by stimulated emission of radiation \\
\hline LUV & large unilamellar vesicle \\
\hline $\mathrm{MgCl}_{2}$ & magnesium chloride \\
\hline MLV & multilamellar vesicle \\
\hline $\mathrm{NaCl}$ & sodium chloride \\
\hline $\mathrm{Na}_{2} \mathrm{HPO}_{4}$ & disodium hydrogenphosphate \\
\hline NOG & $\mathrm{n}$-octyl- $\beta$-D-glucopyranoside \\
\hline PDMS & polydimethylsiloxane \\
\hline Pyranine & 8-Hydroxypyrene-1,3,6-trisulfonic acid trisodium salt \\
\hline RT & room temperature \\
\hline $\mathrm{ROI}$ & region of interest \\
\hline SLB & supported lipid bilayer \\
\hline SNARES & soluble $\mathrm{N}$-ethylmaleimide sensitive factor attachment receptors \\
\hline SUV & small unilamellar vesicle \\
\hline TR & TexasRed ${ }^{\circledR}$ DHPE \\
\hline
\end{tabular}




\subsection{Chemicals and Consumables}

Avidin

A390

ATTO $^{\circledR} 488$ carboxy

A488

A594

BSA

CAS

cap-biotinyl-DOPE

Cellulose-acetate-membranes

CHAPS

Chloroform

Chol

DOPC

DOPE

DOPS

DTT

EDTA

Hellmanex III

HEPES

$\mathrm{KCl}$

$\mathrm{KH}_{2} \mathrm{PO}_{4}$

Methanol

$\mathrm{MgCl}_{2}$

$\mathrm{NaCl}$

$\mathrm{Na}_{2} \mathrm{HPO}_{4}$

NOG

Sylgard ${ }^{\mathrm{TM}} 184$

Glass bottom petri dish $5 \mathrm{~mm}$ Dish, 1.5 \#

Pyranine

Sephadex ${ }^{\circledR}$ illustra NAP-25

Silicon wafers coated with $\mathrm{C}_{4} \mathrm{~F}_{8}$

TexasRed $^{\circledR}$ DHPE

ITO glass slides $25 \times 75 \times 1.1 \mathrm{~mm},(</=10 \mathrm{Ohm} / \mathrm{sq}$.) Präzisions Glas \& Optik GmbH, Iserlohn, Germany

Copper wire, conductive glue, $6 \mathrm{~mm}$
Sigma-Aldrich, Taufkirchen, Germany ATTO-TEC GmbH, Siegen, Germany ATTO-TEC GmbH, Siegen, Germany ATTO-TEC GmbH, Siegen, Germany ATTO-TEC GmbH, Siegen, Germany Sigma-Aldrich, Taufkirchen, Germany Sigma-Aldrich, Taufkirchen, Germany Avanti Polar Lipids, Alabaster, AL, USA Minisart, Sartorius, Göttingen, Germany Sigma-Aldrich, Taufkirchen, Germany VWR International, Darmstadt, Germany Sigma-Aldrich, Taufkirchen, Germany Avanti Polar Lipids, Alabaster, AL, USA Avanti Polar Lipids, Alabaster, AL, USA Avanti Polar Lipids, Alabaster, AL, USA Sigma-Aldrich, Taufkirchen, Germany Sigma-Aldrich, Taufkirchen, Germany Hellma Analytics, Müllheim, Germany Sigma-Aldrich, Taufkirchen, Germany Carl Roth GmbH, Karlsruhe, Germany Sigma-Aldrich, Taufkirchen, Germany Carl Roth GmbH, Karlsruhe, Germany Sigma-Aldrich, Taufkirchen, Germany AppliChem, Darmstadt, Germany Sigma-Aldrich, Taufkirchen, Germany Sigma-Aldrich, Taufkirchen, Germany Dow Corning, Midland, MI, USA MatTek Corporation, Ashland, MA, USA Sigma-Aldrich, Taufkirchen, Germany GE Healthcare, Chicago, USA Caesar, Jülich, Germany Thermo Fischer Scientific, Waltham, MA, USA Fritz Bossert e.K., Hamburg, Germany 


\subsection{Devices}

$\begin{array}{ll}\text { Confocal microscope } & \text { BX61 and a FV1200 CLSM unit from Olympus, Tokio, Japan } \\ \text { Objective } & 60 \text { water or 60× oil objective from Olympus, Tokio, Japan } \\ \text { LASERs } & 405 \mathrm{~nm} \text { (blue), 488 nm (green), 561 nm (red) } \\ \text { Zepto Plasma Generator } & \text { Diener Electronic, Ebhausen, Germany } \\ \text { MilliQ system } & \text { EMD-Millipore, Merck Darmstadt, Germany } \\ \text { Osmometer } & \text { Osmomat 3000, Gonotec, Berlin, Germany } \\ \text { pH-electrode } & \text { il-pht-a120mf-bnc-n, Xylem Analytics, Weilheim, Germany } \\ \text { Rotating evaporator } & \text { Concentrator 5301, Eppendorf, Hamburg } \\ \text { Frequency Generator } & \text { Agilent 33220A Signal Generator, Santa Clara, CA, USA } \\ \text { Extruder } & \text { Lipsofast-Basic, Avestin, Ottawa, Canada } \\ \text { Zetasizer Nano S } & \text { Malvern Instruments, Malvern, UK } \\ \text { Fluorimeter } & \text { FluoroMax, Horiba, Kyoto, Japan } \\ \text { Glass cuvette } & \text { 108F QS, Hellma Analytics, Müllheim, Germany } \\ \text { Spin coating system } & \text { G 3 Series, Special Coating Systems, Indianapolis, IN, USA } \\ \text { Optical microscope } & \text { BX51, Olympus, Tokio, Japan } \\ \text { CCD-camera } & \text { DP71, Olympus, Tokio, Japan } \\ \text { Gas tight syringe, 10 mL } & \text { Hamilton, Reno Nevada, USA } \\ \text { Syringe pump } & \text { Fusion 200, KR Analytical, Sandbach Cheshire, UK } \\ \end{array}$

\subsection{Software}

Image $1.51 \mathrm{~h}$

Matlab2012b

Origin $8.5 \mathrm{G}$

Igor Pro 6.37

Endnote X7

Microsoft Office 2013

ADC/ChemSketch 2015
National Institutes of Health, USA

Mathworks, Natick, MA, USA

OriginLab Cooperation, Northampton, MA, USA

WaveMetrics, Portland, OR, USA

Philadelphia, PA, USA

Redmond, WA, USA

Advanced Chemistry Development Inc., Toronto, Ontario, Canada

\subsection{Coworkers}

Laura Turco and Marco Tarantola from the Max Planck Institute for Dynamics and Self-Organization in Göttingen designed and constructed the milli-fluidic device and provided the PDMS layers for me so that I was able to prepare the thin PDMS sheet on the layers with that the membrane stretching experiments were done. Elena Polo did fundamental work to that topic in her master thesis and investigated the stretchability of PDMS supported lipid bilayers. Jörn Dietz used the milli-fluidic device in his master thesis as a substrate to investigate the possibility to extend the PDMS sheet with incorporated fluorescent beads. He did some fundamental work for the experiment of LUV fusion with dilated lipid bilayers PDMS surfaces. Partho Halder from the group of Reinhard Jahn at the Max-Planck-Institute for Biophysical Chemistry in Göttingen provided the SNARE proteins for all experiments. 


\section{Curriculum vitae}

\section{PERSÖNLICHE DATEN}

Name: Torben-Tobias Kliesch
Geburtsdatum:
07.12 .1986
Geburtsort: Wuppertal
Staatsangehörigkeit: Deutsch

\section{AKADEMISCHE AUSBILDUNG}

2012-2017 Dissertation mit dem Titel: "Investigation of membrane fusion as a function of lateral membrane tension" bei Prof. Dr. Andreas Janshoff am Institut für Physikalische Chemie der Georg-August-Universität Göttingen und dem Graduiertenkolleg der International Max Planck Research School - Physics of Biological and Complex Systems

2010-2012 Chemiestudium an der Georg-August Universität Göttingen mit dem Abschluss Master of Science (M.Sc.)

Masterarbeit: „Membranmechanik einzelner Riesenliposomen mit dem AFM“

2006-2010 Chemiestudium an der Georg-August Universität Göttingen mit dem Abschluss Bachelor of Science (B.Sc.)

Bachelorarbeit: „Proteins and vesicles incorporation into cylindrical nanopores”.

\section{SCHULISCHE AUSBILDUNG}

1997-2006 Gymnasium Nümbrecht: Allgemeine Hochschulreife, Mai 2006.

\section{BESUCHTE KONFERENZEN}

Annual Meeting of the German Biophysical Society 2012, Göttingen, Deutschland GGNB Science Day 2013, Göttingen, Deutschland $9^{\text {th }}$ European Biophysical Congress 2013, Lissabon, Portugal Annual Meeting of the German Biophysical Society 2014, Lübeck, Deutschland Third Infinity 2015, Göttingen, Deutschland $4^{\text {th }}$ GGNB Science Day 2016, Göttingen Deutschland 


\section{Danksagung}

Diese Arbeit wäre ohne die Hilfe vieler Personen, die mich in den letzten Jahren unterstützt haben nicht zustande gekommen.

Ich möchte mich ganz herzliche bei Prof. Dr. Andreas Janshoff für die Betreuung der Doktorarbeit, die interessante Aufgabenstellung und die große Unterstützung in den letzten Jahren bedanken.

Bei Prof. Dr. Tim Salditt und Dr. Florian Rehfeldt möchte ich mich für die konstruktiven Gespräche und Ratschläge in den „Thesis Committee Meetings“ bedanken.

Bei meinen Kooperationspartnern, Laura Turco und Marco Tarantola, möchte ich mich bedanken für die zur Verfügung gestellten Substrate und die Hilfe bei der Entwicklung einer Streckapparatur.

Bei meinen Kooperationspartnern, Partho Halder und Prof. Dr. Reinhard Jahn, bedanke ich mich herzlich für die zur Verfügung gestellten Fusionsproteine ohne die diese Arbeit nicht möglich gewesen wäre. Besonders für die vielen Ideen und Tipps, die während unserer Gespräche entstanden, möchte ich mich bedanken.

Jörn Dietz und Elena Polo danke ich für ihr Engagement bei der Untersuchung der Streckapparatur, die für meine Dissertation grundlegend waren.

Prof. Dr. Burkhard Geil danke ich für die vielen Ideen und konstruktiven Gespräche besonders während unserer Kleingruppentreffen.

Ganz besonders möchte ich mich bei Dr. Ingo Mey bedanken, der mich in allen Situationen immer unterstützt und in schwierigen Zeiten immer wieder aufgebaut hat.

Bei Petra Lappe möchte ich mich herzliche für die Organisation im Arbeitsalltag und für die vielen schönen Gespräche bedanken.

Meinen Bürokollegen Marieelen Oelkers, Hannes Witt und Stefan Nehls danke ich für das besonders freundliche Arbeitsklima und ihre Unterstützung im Labor.

Dem gesamten Arbeitskreis Janshoff danke ich für die Unterstützung in den letzten Jahren und den sehr guten Zusammenhalt. Die vielen gemeinsamen Abende im Kaffeeraum, in Restaurants oder auf dem Weihnachtsmarkt waren sehr schöne Erlebnisse, die einem den doch oft schweren Arbeitsalltag erleichterten. Ich werde die vielen politischen und ideologischen Diskussionen mit euch vermissen.

Meinem guten Freund, Christian Volkmann, möchte ich besonders für sein offenes Ohr und die vielen wichtigen und konstruktiven Gespräche danke. Du bist ein sehr guter Freund auf den ich mich immer verlassen kann.

Ein ganz besonderer Dank gilt meinen Eltern und Geschwistern, die mich während meiner Doktorarbeit immer wieder vor allem in schweren Zeit unterstützt und aufgebaut haben. Ohne euch hätte ich es nicht so weit geschafft. Vielen Dank, für die aufbauenden Worte und vielen Gebete. 